\title{
Performance of CORBA-Based Client-Server Architectures
}

\author{
by \\ Istabrak N. Abdul-Fatah
}

A thesis submitted to the Faculty of Graduate Studies and Research

in partial fulfillment of

the requirements for the degree of

Master of Engineering

Ottawa-Carleton Institute for Electrical Engineering

Faculty of Engineering

Department of Systems and Computer Engineering

Carleton University

Ottawa, Ontario, Canada, K1S 5B6

May 12, 1997

(C) Copyright 1997, Istabrak N. Abdul-Fatah 
National Library

of Canada

Acquisitions and Bibliographic Services

395 Wellington Street Ottawa ON K1A ONA Canada
Bibliothèque nationale du Canada

Acquisitions et services bibliographiques

395, rue Wellington

Ottawa ON K1A ON4

Canada
Your the votre rifterence

Our tie Norre relerence
The author has granted a nonexclusive licence allowing the National Library of Canada to reproduce, loan, distribute or sell copies of this thesis in microform, paper or electronic formats.
L'auteur a accordé une licence non exclusive permettant à la Bibliothèque nationale du Canada de reproduire, prêter, distribuer ou vendre des copies de cette thèse sous la forme de microfiche/film, de reproduction sur papier ou sur format électronique.

L'auteur conserve la propriété du droit d'auteur qui protège cette thèse. $\mathrm{Ni}$ la thèse ni des extraits substantiels de celle-ci ne doivent être imprimés ou autrement reproduits sans son autorisation. 
To My Mother and in Memory of My Father 


\begin{abstract}
The notion of middleware has been introduced to provide interoperability as well as transparent location of servers in heterogeneous client-server environments. Careful consideration of system architecture is required to achieve high performance. Based on implementation and measurements made on the system this research is concerned with the impact of client-server interaction architecture on the performance of a CORBA System. Using a commercially available CORBA compliant ORB software called ORBeline, four different architectures, the Handle Driven ORB, the Forwarding ORB, the Process Planner, and the Adaptive ORB have been designed and implemented for client-server interaction on a network of workstations. High level descriptions provided in the literature were used for building the first three architectures whereas the fourth is a new architecture proposed in this thesis. The measurements show that the difference among the performances of these architectures change with a change in the workload. The research will report on the relative performances of the four architectures under different workload conditions. The results provide insights into system behavior and guidelines for designers as well as users of systems. In particular the impact of inter-node delays, message size, request service times and the system load threshold level on the latency and scalability attributes of these architectures are analyzed. A discussion of how agent cloning can improve system performance is also included.
\end{abstract}




\section{Acknowledgments}

I would like to express my sincere thanks to my supervisor Professor Shikharesh Majumdar for his invaluable patience, support and guidance during the past two years.

Also, I would like to convey my thanks to Professor Dorina Pertiu for all her encouragement. The support of my RADS colleagues especially Guru, Fahim, Cheryl, Alex, Hesham, Peter, Leslie, and our system administrator, Naren, is highly acknowledged.

I would like to express my deep appreciation and thanks to my wife, Manal, and my daughters, Ammanie and Tara for their patience, understanding and strong support without which the research would not have been possible.

Last but not least, I would like to acknowledge the National Sciences and Engineering Research Council (NSERC) for funding this research, the Telecommunication Research Institute of Ontario (TRIO) for offering the RADS lab equipment used in this research, and Visigenic Software for offering their ORBeline software used in this research. 


\section{Table of Contents}

Chapter 1 Introduction 1

1.1 An Overview of Distributed Systems 1

1.2 Distributed Object Computing (DOC) 2

1.3 Motivation for the Thesis 3

1.4 Goals of the Thesis 3

1.5 Thesis Outline 5

Chapter 2 Background 6

2.1 DOC System Design and Analysis 6

2.2 Development Techniques for Distributed Applications 8

2.2.1 Lower Level Programming Interfaces 9

2.2.2 Transport Layer Interfaces 9

2.2.3 C++ Wrappers Interfaces 11

2.2.4 RPC Toolkit Interfaces $\quad 15$

\section{Chapter 3 CORBA Overview 16}

$\begin{array}{lll}3.1 & \text { Introduction } & 16\end{array}$

3.2 The CORBA Object Model 17

3.3 The Common Object Request Broker Architecture $\begin{array}{ll}\text { (CORBA) } & 18\end{array}$

$\begin{array}{lll}3.3 .1 & \text { ORB Core } & 20\end{array}$

3.3.2 The ORB Interface 20

3.3.3 CORBA Interface Definition Language (IDL) 20 
3.3.3.2 The Static IDL Skeleton 24

3.3.4 Dynamic Invocation Interface (DII) 24

3.3.5 Dynamic Skeleton Interface (DSI) 25

3.3.6 Object Adapter (OA) 26

3.4 CORBA, a Development Technique for DOC Systems 26

3.5 The Distributed Computing Environment (DCE) 28

3.6 CORBA and DCE 30

Chapter 4 The Request Broker Architectures 33

$\begin{array}{lll}\text { 4.1 Definitions } & 33\end{array}$

4.2 CORBA Applications Development Steps 35

4.2.1 The IDL mapping approach 35

4.2.2 External Installation 36

4.3 The Handle-Driven ORB (H-ORB) Architecture 38

4.3.1 Definition 38

4.3.2 The H-ORB Scenario 40

4.3.2.1 The Client 41

4.3.2.2 The Agent 43

4.3.2.3 The Server (Object Implementation) 44

4.4 The Forwarding ORB (F-ORB) Architecture 45

4.4.1 Definition 45

4.4.2 The F-ORB Scenario 46

4.4.2.1 The Client 46

4.4.2.2 The Agent 48 
4.4.2.3 The Server (Object Implementation) 49

4.5 The Process Planner ORB (P-ORB) Architecture 52

4.5.1 Definitions 53

4.5.2 The P-ORB Scenario 54

4.5.2.1 The Client 54

4.5.2.2 The Agent 56

4.5.2.3 The Server (Object Implementation) 57

\section{Chapter 5 Comparison of System Performance 60}

$\begin{array}{lll}5.1 & \text { Case Study } & 60\end{array}$

$\begin{array}{lll}\text { 5.1.1 The Workload } & 60\end{array}$

5.1.2 Experimental Environment 63

5.1.2.1 The Handle-Driven ORB H-ORB System 64

5.1.2.2 The Forwarding ORB F-ORB System 67

5.1.2.3 The Process Planner P-ORB System 76

5.2 Experimental Results 83

5.2.1 Experiment Control and Data Logging 86

5.2.2 Elapsed and Cpu Time Measurements 87

5.2.3 The Performance of the H-ORB on a Single Workstation 88

5.2.4 The Effect of Message Size 91

5.2.4.1 No Cloning (Single Agent) Architectures 92

5.2.4.2 Four-Degree Cloned Architectures 99

5.2.4.3 Eight-Degree Cloned Architectures 103

5.2.5 The Impact of the Server Demand 107

5.2.5.1 No Cloning (Single Agent) Architectures 108 
5.2.5.2 Four-Degree Cloned Architectures

113

5.2.5.3 Eight-Degree Cloned Architectures

120

5.2.6 The Effect of Inter-Node Delay

121

5.2.6.1 No Cloning (Single Agent) Architectures 122

5.2.6.2 Four-Degree Cloned Architectures 126

5.2.6.3 Eight-Degree Cloned Architectures $\quad 130$

5.3 Discussion of Results 134

5.3.1 The Message Size L 134

5.3.2 The Server Demand $\mathrm{S}_{\mathrm{A}} / \mathrm{S}_{\mathrm{B}} \quad 135$

5.3.3 The Inter-Node Delays D 136

$\begin{array}{lll}\text { Chapter } 6 & \text { The Adaptive ORB (A-ORB) } & 138\end{array}$

$\begin{array}{lll}6.1 & \text { Motivation } & 138\end{array}$

6.2 Definition 139

6.2.1 The A-ORB Scenario 139

6.2.1.1 The Client 141

6.2.1.2 The Agent's Dispatcher 142

6.2.1.3 The Agent 144

6.2.1.4 The Server (Object Implementation) 145

$\begin{array}{lll}6.3 & \text { Case Study } & 147\end{array}$

$\begin{array}{ll}\text { 6.3.1 The Workload } & 147\end{array}$

6.3.2 The Experimental Environment 148

6.3.2.1 The Adaptive ORB A-ORB System 149

6.4 Experimental Results 155

6.4.1 The Effect of the Inter-Node Delay 156 
$\begin{array}{lll}7.1 & \text { Summary } & 166\end{array}$

7.2 Gained Insights 166

$\begin{array}{lll}7.3 & \text { Derived Guidelines } & 168\end{array}$

$\begin{array}{lll}7.4 & \text { Thesis Contributions } & 168\end{array}$

$\begin{array}{lll}7.5 & \text { Future Research } & 169\end{array}$

Supplementary Results for the Message Size Set of Experiments

\section{Appendix B}

Supplementary Results for the Server Demand Set of Experiments

\section{Appendix C}

Supplementary Results for the Inter-Node Delay Set of Experiments

\section{Appendix D}

Supplementary Results for the A-ORB Set of Experiments 


\section{List of Figures}

Figure 2-1: $\quad$ IPC Interfaces in Modem OS Platforms $\ldots \ldots \ldots \ldots \ldots \ldots$

Figure 2-2: IPC SAP Class Category Relationships ............... 12

Figure 2-3: The IPC SAP C + Wrapper $\ldots \ldots \ldots \ldots \ldots \ldots \ldots \ldots \ldots \ldots$

Figure 3-1: The Structure of the Object Request Broker ............. 19

Figure 3-2: $\quad$ An example of a CORBA IDL source code $\ldots \ldots \ldots \ldots \ldots . \ldots 22$

Figure 3-3: Partial $\mathrm{C}++$ code generated by ORBeline IDL compiler for the source code of Figure 3-2 showing mapping of parameters and a Stub code. . . . . . . . . . . . . . . . . 23

Figure 3-4: One of the Static Skeletons generated by ORBeline IDL compiler for the source code of Figure $3-2 \ldots \ldots \ldots \ldots \ldots 24$

Figure 3-5: The DCE Components and their dependency relations ........ 30

Figure 4-1: General CORBA Applications Development Phases ......... 37

Figure 4-2: A high level interaction description of the H-ORB Architecture. . . . . . . . . . . . . . . . . . . . . . 39

Figure 4-3: List of Object and symbols used in the execution scenario diagrams. . . . . . . . . . . . . . . . . 40

Figure 4-4: The H-ORB Execution Scenario using Client Static Invocation and Persistent Servers $\ldots \ldots \ldots \ldots \ldots \ldots \ldots \ldots 42$

Figure 4-5: A high level interaction description of the F-ORB

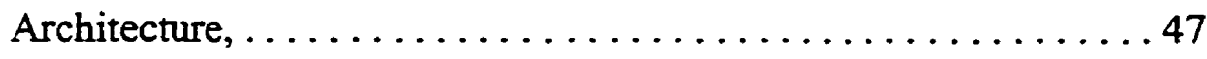

Figure 4-6: The F-ORB Execution Scenario using Client Static Invocation and Persistent Servers $\ldots \ldots \ldots \ldots \ldots \ldots \ldots . \ldots . \ldots$

Figure 4-7: A composite request and its constituent components. ........52 
Figure 4-8: A high level interaction description of the P-ORB

Architecture................................. 55

Figure 4-9: The P-ORB Execution Scenario using Client Static Invocation and Persistent Servers $\ldots \ldots \ldots \ldots \ldots \ldots \ldots . \ldots . \ldots$

Figure 5-1: The H-ORB Case Study Request Sequence Path $\ldots \ldots \ldots \ldots 66$

Figure 5-2: The F-ORB.V1 Case Study Request Sequence Path .......... 70

Figure 5-3: The F-ORB.V2 Case Study Request Sequence Path .......... 74

Figure 5-4: The P-ORB Deadlock Scenario, Case $-1 \ldots \ldots \ldots . \ldots 79$

Figure 5-5: The P-ORB Deadlock Scenario, Case $-2 \ldots \ldots \ldots \ldots . \ldots 80$

Figure 5-6: The P-ORB Case Study Request Sequence Path $\ldots \ldots \ldots \ldots . \ldots 1$

Figure 5-7: The Process \& Cpu Utilization concepts $\ldots \ldots \ldots \ldots \ldots \ldots . \ldots 4$

Figure 5-8: The H-ORB Execution Scenario using Client Static Invocation and Persistent Servers $\ldots \ldots \ldots \ldots \ldots \ldots . \ldots 90$

Figure 5-9: The Response Time of the H-ORB, the F-ORB and the P-ORB with $\mathrm{L}=4800$ Bytes, and no Cloning. ..........93 93

Figure 5-10: The Throughput of the H-ORB, the F-ORB and the P-ORB with $\mathrm{L}=4800$ Bytes, and no Cloning. . . . . . . . 93

Figure 5-11: Cpu and Process Utilizations of the H-ORB with $\mathrm{L}=4800$ Bytes and No Cloning. ................. 94

Figure 5-12: Cpu \& Process Utilizations of the F-ORB with $\mathrm{L}=4800$ Bytes and No Cloning. ........................... 94

Figure 5-13: Cpu \& Process Utilizations of the P-ORB with $L=4800$ Bytes and No Cloning. ............................ 95

Figure 5-14: The Response Time of the H-ORB, the F-ORB and the P-ORB with $\mathrm{L}=4800$ Bytes, and 4 Cloning Degrees. . . . . . . . 100 
Figure 5-15: The Throughput of the H-ORB, the F-ORB and the P-ORB with $\mathrm{L}=4800$ Bytes, and 4 Cloning Degrees $\ldots \ldots \ldots \ldots 100$

Figure 5-16: Cpu \& Process Utilizations of the F-ORB with L=4800 Bytes and 4 Cloning Degrees. ..................... 102

Figure 5-17: Cpu \& Process Utilizations of the P-ORB with $\mathrm{L}=4800$ Bytes and 4 Cloning Degrees. . . . . . . . . . . . . . 102

Figure 5-18: The Response Time of the H-ORB, the F-ORB and the P-ORB with $\mathrm{L}=4800$ Bytes, and 8 Cloning Degrees. . . . . . . . 104

Figure 5-19: The Throughput of the H-ORB, the F-ORB and the P-ORB with $L=4800$ Bytes, and 8 Cloning Degrees. . . . . . . 104

Figure 5-20: Cpu \& Process Utilizations of the F-ORB with $\mathrm{L}=4800$ Bytes and 8 Cloning Degrees. . . . . . . . . . . . . . 105

Figure 5-21: Cpu \& Process Utilizations of the P-ORB with $L=4800$ Bytes and 8 Cloning Degrees.

Figure 5-22: The Response Time of the $\mathrm{H}-\mathrm{ORB}$, the F-ORB and the P-ORB with $\mathrm{S}_{\mathrm{A}} / \mathrm{S}_{\mathrm{B}}=10 / 15 \mathrm{~ms}$, and no Cloning. ............

Figure 5-23: The Throughput of the H-ORB, the F-ORB and the P-ORB with $\mathrm{S}_{\mathrm{A}} / \mathrm{S}_{\mathrm{B}}=10 / 15 \mathrm{~ms}$, and no Cloning. . . . . . . . . . . $\ldots 10$

Figure 5-24: Cpu and Process Utilizations of the $H-O R B$ with $\mathrm{S}_{\mathrm{A}} / \mathrm{S}_{\mathrm{B}}=10 / 15 \mathrm{~ms}$, and no Cloning.

Figure 5-25: Cpu \& Process Utilizations of the F-ORB with $\mathrm{S}_{\mathrm{A}} / \mathrm{S}_{\mathrm{B}}=10 / 15 \mathrm{~ms}$, and no Cloning

Figure 5-26: Cpu \& Process Utilizations of the P-ORB with $S_{A} / S_{B}=10 / 15 \mathrm{~ms}$, and no Cloning.

Figure 5-27: The Response Time of the H-ORB, the F-ORB and the P-ORB 
with $\mathrm{S}_{\mathrm{A}} / \mathrm{S}_{\mathrm{B}}=250 / 375 \mathrm{~ms}$, and no Cloning. .............. 114

Figure 5-28: The Throughput of the H-ORB, the F-ORB and the P-ORB

with $\mathrm{S}_{\mathrm{A}} / \mathrm{S}_{\mathrm{B}}=250 / 375 \mathrm{~ms}$, and no Cloning. ............... 114

Figure 5-29: Cpu and Process Utilizations of the H-ORB with

$\mathrm{S}_{\mathrm{A}} / \mathrm{S}_{\mathrm{B}}=250 / 375 \mathrm{~ms}$, and no Cloning.

Figure 5-30: Cpu \& Process Utilizations of the F-ORB with

$\mathrm{S}_{\mathrm{A}} / \mathrm{S}_{\mathrm{B}}=250 / 375 \mathrm{~ms}$, and no Cloning.

Figure 5-31: Cpu \& Process Utilizations of the P-ORB with

$\mathrm{S}_{\mathrm{A}} / \mathrm{S}_{\mathrm{B}}=250 / 375 \mathrm{~ms}$, and no Cloning.

Figure 5-32: The Response Time of the H-ORB, the F-ORB and the P-ORB with $\mathrm{S}_{\mathrm{A}} / \mathrm{S}_{\mathrm{B}}=10 / 15 \mathrm{~ms}$, and 4 Cloning Degrees............. 118

Figure 5-33: The Throughput of the H-ORB, the F-ORB and the P-ORB with $\mathrm{S}_{\mathrm{A}} / \mathrm{S}_{\mathrm{B}}=10 / 15 \mathrm{~ms}, 4$ Cloning Degrees. . . . . . . . . . . . 118

Figure 5-34: Cpu \& Process Utilizations of the F-ORB with

$\mathrm{S}_{\mathrm{A}} / \mathrm{S}_{\mathrm{B}}=10 / 15 \mathrm{~ms}$, and 4 Cloning Degrees.

Figure 5-35: Cpu \& Process Utilizations of the P-ORB with

$\mathrm{S}_{\mathrm{A}} / \mathrm{S}_{\mathrm{B}}=10 / 15 \mathrm{~ms}$, and 4 Cloning Degrees.

Figure 5-36: The Response Time of the H-ORB, the F-ORB and the P-ORB with $\mathrm{D}=250 \mathrm{~ms}$, and no Cloning.

Figure 5-37: The Throughput of the H-ORB, the F-ORB and the P-ORB with $\mathrm{D}=250 \mathrm{~ms}$, and no Cloning.

Figure 5-38: $\mathrm{Cpu}$ and Process Utilizations of the H-ORB with $\mathrm{D}=250 \mathrm{~ms}$, and no Cloning.

Figure 5-39: Cpu \& Process Utilizations of the F-ORB with $D=250 \mathrm{~ms}$, and no Cloning. 
Figure 5-40: Cpu \& Process Utilizations of the P-ORB with $\mathrm{D}=250 \mathrm{~ms}$, and no Cloning Degrees. . . . . . . . . . . . . . . 125

Figure 5-41: The Response Time of the H-ORB, the F-ORB and the P-ORB with $\mathrm{D}=250 \mathrm{~ms}$, and 4 Cloning Degrees. . . . . . . . . . 128

Figure 5-42: The Throughput of the H-ORB, the F-ORB and the P-ORB with $\mathrm{D}=250 \mathrm{~ms}$, and 4 Cloning Degrees. . . . . . . . . . . 128

Figure 5-43: Cpu \& Process Utilizations of the F-ORB with D $=250 \mathrm{~ms}$, and 4 Cloning Degrees.

Figure 5-44: Cpu \& Process Utilizations of the P-ORB with D $=250 \mathrm{~ms}$, and 4 Cloning Degrees.

Figure 5-45: The Response Time of the H-ORB, the F-ORB and the P-ORB with $\mathrm{D}=250 \mathrm{~ms}$, and 8 Cloning.

Figure 5-46: The Throughput of the H-ORB, the F-ORB and the P-ORB with $\mathrm{D}=250 \mathrm{~ms}$, and 8 Cloning.

Figure 5-47: Cpu \& Process Utilizations of the F-ORB with $D=250 \mathrm{~ms}$, and 8 Cloning Degrees. . . . . . . . . . . . . . . . . 132

Figure 5-48: Cpu \& Process Utilizations of the P-ORB with $D=250 \mathrm{~ms}$, and 8 Cloning Degrees.

Figure 6-1: A high level interaction description of the A-ORB Architecture .. 140

Figure 6-2: The A-ORB Execution Scenario using Client Static Invocation and Persistent Servers $\ldots \ldots \ldots \ldots \ldots \ldots \ldots \ldots \ldots \ldots$

Figure 6-3: The A-ORB Case Study Request Sequence Path $\ldots \ldots \ldots \ldots \ldots 151$

Figure 6-4: The Response Time of the H-ORB, the F-ORB and the A-ORB with Equidistant Nodes. . . . . . . . . . . . . . . . . 158

Figure 6-5: The Throughput of the H-ORB, the F-ORB and the A-ORB, with 
Equidistant Nodes. . . . . . . . . . . . . . . . . . 158

Figure 6-6: The Process and Cpu Utilizations of the A-ORB (QL=0) $\ldots \ldots . .159$

Figure 6-7: The Process and Cpu Utilizations of the A-ORB (QL=1) . . . . 159

Figure 6-8: The Process and Cpu Utilizations of the A-ORB (QL=3) $\ldots \ldots \ldots 160$

Figure 6-9: The Response Time of the H-ORB, the F-ORB and the A-ORB with Non Equidistant Nodes. . . . . . . . . . . . . . . 162

Figure 6-10: The Throughput of the H-ORB, the F-ORB and the A-ORB with Non Equidistant Nodes. . . . . . . . . . . . . . . . 162

Figure 6-11: The Process and Cpu Utilizations of the F-ORB with Non Equidistant Nodes.

Figure 6-12: The Process and Cpu Utilizations of the A-ORB with Non Equidistant Nodes $(\mathrm{QL}=0)$

Figure 6-13: The Process and Cpu Utilizations of the A-ORB with Non Equidistant Nodes $(\mathrm{QL}=1) \ldots \ldots \ldots \ldots \ldots \ldots \ldots \ldots \ldots \ldots \ldots \ldots \ldots \ldots \ldots$

Figure 6-14: The Process and Cpu Utilizations of the A-ORB with Non Equidistant Nodes $(\mathrm{QL}=3) \ldots \ldots \ldots \ldots \ldots \ldots \ldots \ldots \ldots \ldots \ldots \ldots \ldots \ldots$

Figure A-1: The Response Time of the H-ORB, the F-ORB.V2 and the P-ORB with $L=4800$ Bytes, and No Cloning. . . . . . . . A-2

Figure A-2: The Throughput of the H-ORB, the F-ORB.V2 and the P-ORB with $\mathrm{L}=4800$ Bytes, and No Cloning. ...............

Figure A-3: Cpu \& Process Utilizations of the F-ORB.V2 with L=4800 Bytes and No cloning. $\ldots \ldots \ldots \ldots \ldots \ldots \ldots \ldots \ldots \ldots \ldots \ldots \ldots \ldots \ldots \ldots \ldots$

Figure A-4: The Response Time of the H-ORB, the F-ORB and the P-ORB with $\mathrm{L}=9600$ Bytes, and No Cloning. . . . . . . . . . . A-4

Figure A-5: The Throughput of the H-ORB, the F-ORB and the P-ORB with 
$\mathrm{L}=9600$ Bytes, and No Cloning.

Figure A-6: Cpu and Process Utilizations of the H-ORB with $\mathrm{L}=9600$ Bytes and No cloning.

Figure A-7: Cpu \& Process Utilizations of the F-ORB with L=9600 Bytes and No cloning.

Figure A-8: Cpu \& Process Utilizations of the P-ORB with L=9600 Bytes and No cloning.

Figure A-9: The Response Time of the H-ORB, the F-ORB and the P-ORB with $\mathrm{L}=19200$ Bytes, and No Cloning.

Figure A-10: The Throughput of the H-ORB, the F-ORB and the P-ORB with $\mathrm{L}=19200$ Bytes, and No Cloning.

Figure A-11: Cpu and Process Utilizations of the H-ORB with $\mathrm{L}=19200$ Bytes and No cloning.

Figure A-12: Cpu \& Process Utilizations of the F-ORB with $\mathrm{L}=19200$ Bytes and No cloning.

Figure A-13: Cpu \& Process Utilizations of the P-ORB with $L=19200$ Bytes and No Cloning.

Figure A-14: The Response Time of the H-ORB, the F-ORB and the P-ORB with $\mathrm{L}=9600$ Bytes, and 4 Cloning Degrees.

Figure A-15: The Throughput of the H-ORB, the F-ORB and the P-ORB with $L=9600$ Bytes, and 4 Cloning Degrees.

Figure A-16: Cpu \& Process Utilizations of the F-ORB with L=9600 Bytes and 4 Cloning Degrees.

Figure A-17: Cpu \& Process Utilizations of the P-ORB with L=9600 Bytes and 4 Cloning Degrees. 
Figure A-18: The Response Time of the H-ORB, the F-ORB and the P-ORB with $\mathrm{L}=19200$ Bytes, and 4 Cloning Degrees. ........... A-12

Figure A-19: The Throughput of the H-ORB, the F-ORB and the P-ORB with $\mathrm{L}=19200$ Bytes, and 4 Cloning Degrees.

Figure A-20: Cpu \& Process Utilizations of the F-ORB with $L=19200$ Bytes and 4 Cloning Degrees. . . . . . . . .

Figure A-21: Cpu \& Process Utilizations of the P-ORB with L=19200 Bytes and 4 Cloning Degrees.

Figure A-22: The Response Time of the H-ORB, the F-ORB and the P-ORB with $\mathrm{L}=9600$ Bytes, and 8 Cloning Degrees. ............ A-14

Figure A-23: The Throughput of the H-ORB, the F-ORB and the P-ORB with $\mathrm{L}=9600$ Bytes, and 8 Cloning Degrees.

Figure A-24: Cpu \& Process Utilizations of the F-ORB with L=9600 Bytes and 8 Cloning Degrees. . . . . . . . .

Figure A-25: Cpu \& Process Utilizations of the P-ORB with $L=9600$ Bytes and 8 Cloning Degrees.

Figure A-26: The Response Time of the H-ORB, the F-ORB and the P-ORB with $\mathrm{L}=19200$ Bytes, and 8 Cloning Degrees. ............ A-16

Figure A-27: The Throughput of the H-ORB, the F-ORB and the P-ORB with $\mathrm{L}=19200$ Bytes, and 8 Cloning Degrees.

Figure A-28: Cpu \& Process Utilizations of the F-ORB with L=19200 Bytes and 8 Cloning Degrees. ................. 17

Figure A-29: Cpu \& Process Utilizations of the P-ORB with $L=19200$ Bytes and 8 Cloning Degrees.

Figure B-1: The Response Time of the H-ORB, the F-ORB and the P-ORB 
with $\mathrm{S}_{\mathrm{A}} / \mathrm{S}_{\mathrm{B}}=50 / 75 \mathrm{~ms}$, and no Cloning. ...............

Figure B-2: The Throughput of the H-ORB, the F-ORB and the P-ORB with

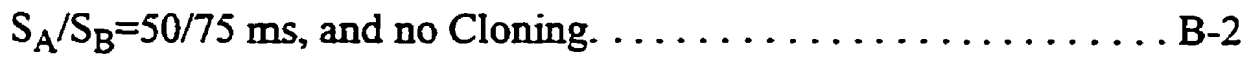

Figure B-3: Cpu and Process Utilizations of the H-ORB with $\mathrm{S}_{\mathrm{A}} / \mathrm{S}_{\mathrm{B}}=50 / 75 \mathrm{~ms}$, and no Cloning.

Figure B-4： Cpu \& Process Utilizations of the F-ORB with $\mathrm{S}_{\mathrm{A}} / \mathrm{S}_{\mathrm{B}}=50 / 75 \mathrm{~ms}$, and no Cloning.

Figure B-5: Cpu \& Process Utilizations of the P-ORB with $\mathrm{S}_{\mathrm{A}} / \mathrm{S}_{\mathrm{B}}=50 / 75 \mathrm{~ms}$, and no Cloning.

Figure B-6: $\quad \mathrm{Cpu}$ and Process Utilizations of the H-ORB with $\mathrm{S}_{\mathrm{A}} / \mathrm{S}_{\mathrm{B}}=50 / 75 \mathrm{~ms}$, and 4 Cloning degrees. ............

Figure B-7: The Throughput of the H-ORB, the F-ORB and the P-ORB with $\mathrm{S}_{\mathrm{A}} / \mathrm{S}_{\mathrm{B}}=50 / 75 \mathrm{~ms}$, and 4 Cloning degrees.

Figure B-8: Cpu \& Process Utilizations of the F-ORB with $\mathrm{S}_{\mathrm{A}} / \mathrm{S}_{\mathrm{B}}=50 / 75 \mathrm{~ms}$, and 4 Cloning degrees.

Figure B-9: Cpu \& Process Utilizations of the P-ORB with $\mathrm{S}_{\mathrm{A}} / \mathrm{S}_{\mathrm{B}}=50 / 75 \mathrm{~ms}$, and 4 Cloning degrees.

Figure B-10: The Response Time of the H-ORB, the F-ORB and the P-ORB with $\mathrm{S}_{\mathrm{A}} / \mathrm{S}_{\mathrm{B}}=250 / 375 \mathrm{~ms}$, and 4 Cloning degrees.

Figure B-11: The Throughput of the H-ORB, the F-ORB and the P-ORB with $\mathrm{S}_{\mathrm{A}} / \mathrm{S}_{\mathrm{B}}=250 / 375 \mathrm{~ms}$, and 4 Cloning degrees.

Figure B-12: Cpu \& Process Utilizations of the F-ORB with $\mathrm{S}_{\mathrm{A}} / \mathrm{S}_{\mathrm{B}}=250 / 375 \mathrm{~ms}$, and 4 Cloning Degrees. . . . . . . . . B 8

Figure B-13: Cpu \& Process Utilizations of the P-ORB with $\mathrm{S}_{\mathrm{A}} / \mathrm{S}_{\mathrm{B}}=250 / 375 \mathrm{~ms}$, and 4 Cloning Degrees. B-8 
Figure B-14: The Response Time of the H-ORB, the F-ORB and the P-ORB with $\mathrm{S}_{\mathrm{A}} / \mathrm{S}_{\mathrm{B}}=10 / 15 \mathrm{~ms}$, and 8 Cloning Degrees. . . . . . . . . B-9

Figure B-15: The Throughput of the H-ORB, the F-ORB and the P-ORB with $\mathrm{S}_{\mathrm{A}} / \mathrm{S}_{\mathrm{B}}=10 / 15 \mathrm{~ms}$, and 8 Cloning Degrees. . . . . . . . . .

Figure B-16: Cpu \& Process Utilizations of the F-ORB with $\mathrm{S}_{\mathrm{A}} / \mathrm{S}_{\mathrm{B}}=10 / 15 \mathrm{~ms}$, and 8 Cloning Degrees.

Figure B-17: Cpu \& Process Utilizations of the P-ORB with $\mathrm{S}_{\mathrm{A}} / \mathrm{S}_{\mathrm{B}}=10 / 15 \mathrm{~ms}$, and 8 Cloning Degrees.

Figure B-18: The Response Time of the H-ORB, the F-ORB and the P-ORB with $\mathrm{S}_{\mathrm{A}} / \mathrm{S}_{\mathrm{B}}=50 / 75 \mathrm{~ms}$, and 8 Cloning degrees.

Figure B-19: The Throughput of the $\mathrm{H}-\mathrm{ORB}$, the F-ORB and the P-ORB with $\mathrm{S}_{\mathrm{A}} / \mathrm{S}_{\mathrm{B}}=50 / 75 \mathrm{~ms}$, and 8 Cloning degrees.

Figure B-20: Cpu \& Process Utilizations of the F-ORB with $\mathrm{S}_{\mathrm{A}} / \mathrm{S}_{\mathrm{B}}=50 / 75 \mathrm{~ms}$, and 8 Cloning degrees.

Figure B-21: Cpu \& Process Utilizations of the P-ORB with $\mathrm{S}_{\mathrm{A}} / \mathrm{S}_{\mathrm{B}}=50 / 75 \mathrm{~ms}$, and 8 Cloning degrees.

Figure B-22: The Response Time of the H-ORB, the F-ORB and the P-ORB with $\mathrm{S}_{\mathrm{A}} / \mathrm{S}_{\mathrm{B}}=250 / 375 \mathrm{~ms}$, and 8 Cloning degrees.

Figure B-23: The Throughput of the H-ORB, the F-ORB and the P-ORB with $\mathrm{S}_{\mathrm{A}} / \mathrm{S}_{\mathrm{B}}=250 / 375 \mathrm{~ms}$, and 8 Cloning degrees.

Figure B-24: Cpu \& Process Utilizations of the F-ORB with $\mathrm{S}_{\mathrm{A}} / \mathrm{S}_{\mathrm{B}}=250 / 375 \mathrm{~ms}$, and 8 Cloning degrees.

Figure B-25: Cpu \& Process Utilizations of the P-ORB with $\mathrm{S}_{\mathrm{A}} / \mathrm{S}_{\mathrm{B}}=250 / 375 \mathrm{~ms}$, and 8 Cloning degrees. B-14

Figure C-1: The Response Time of the H-ORB, the F-ORB.V2 and the P-ORB 
with $\mathrm{D}=250 \mathrm{~ms}$, and no Cloning. ...............

Figure C-2: The Throughput of the H-ORB, the F-ORB.V2 and the P-ORB with $\mathrm{D}=250 \mathrm{~ms}$, and no Cloning. ................

Figure C-3: Cpu \& Process Utilizations of the F-ORB.V2 with D $=250 \mathrm{ms,}$ and no Cloning. $\ldots \ldots \ldots \ldots \ldots \ldots \ldots \ldots \ldots \ldots \ldots \ldots \ldots \ldots \ldots \ldots \ldots$

Figure C-4: The Response Time of the H-ORB, the F-ORB and the P-ORB with $\mathrm{D}=500 \mathrm{~ms}$, and no Cloning.

Figure C-5: The Throughput of the H-ORB, the F-ORB and the P-ORB with $\mathrm{D}=500 \mathrm{~ms}$, and no Cloning.

Figure C-6: Cpu and Process Utilizations of the H-ORB with $D=500 \mathrm{~ms}$, and no Cloning.

Figure C-7: Cpu \& Process Utilizations of the F-ORB with $D=500 \mathrm{~ms}$, and no Cloning.

Figure C-8: Cpu \& Process Utilizations of the P-ORB with $D=500 \mathrm{~ms}$, and no Cloning Degrees.

Figure C-9: The Response Time of the H-ORB, the F-ORB and the P-ORB with $\mathrm{D}=1000 \mathrm{~ms}$, and no Cloning.

Figure $\mathrm{C}-10$ : The Throughput of the H-ORB, the F-ORB and the P-ORB with $\mathrm{D}=1000 \mathrm{~ms}$, and no Cloning.

Figure C-1 1: Cpu and Process Utilizations of the H-ORB with D $=1000 \mathrm{~ms}$, and no Cloning. C-8

Figure C-12: Cpu \& Process Utilizations of the F-ORB with $\mathrm{D}=1000 \mathrm{~ms}$, and no Cloning.

Figure C-13: Cpu \& Process Utilizations of the P-ORB with D $=1000 \mathrm{~ms}$, and no Cloning Degrees. 
Figure C-14: The Response Time of the H-ORB, the F-ORB and the P-ORB with $\mathrm{D}=500 \mathrm{~ms}$, and 4 Cloning degrees.

Figure C-15: The Throughput of the H-ORB, the F-ORB and the P-ORB with $\mathrm{D}=500 \mathrm{~ms}$, and 4 Cloning degrees.

Figure C-16: Cpu \& Process Utilizations of the F-ORB with $D=500 \mathrm{~ms}$, and 4 Cloning degrees.

Figure C-17: Cpu \& Process Utilizations of the P-ORB with D $=500 \mathrm{~ms}$, and 4 Cloning Degrees.

Figure C-18: The Response Time of the H-ORB, the F-ORB and the P-ORB with

$\mathrm{D}=1000 \mathrm{~ms}$, and 4 Cloning degrees.

Figure C-19: The Throughput of the H-ORB, the F-ORB and the P-ORB with $\mathrm{D}=1000 \mathrm{~ms}$, and 4 Cloning degrees.

Figure C-20: Cpu \& Process Utilizations of the F-ORB with $D=1000 \mathrm{~ms}$, and 4 Cloning degrees.

Figure C-21: Cpu \& Process Utilizations of the P-ORB with $D=1000 \mathrm{~ms}$, and 4 Cloning degrees.

Figure C-22: The Response Time of the H-ORB, the F-ORB and the P-ORB with $\mathrm{D}=500 \mathrm{~ms}$, and 8 Cloning degrees.

Figure C-23: The Throughput of the H-ORB, the F-ORB and the P-ORB with $\mathrm{D}=500 \mathrm{~ms}$, and 8 Cloning degrees.

Figure C-24: Cpu \& Process Utilizations of the F-ORB with D $=500 \mathrm{~ms}$, and 8 Cloning degrees.

Figure C-25: Cpu \& Process Utilizations of the P-ORB with $D=500 \mathrm{~ms}$, and 8 Cloning degrees.

Figure C-26: The Response Time of the H-ORB, the F-ORB and the P-ORB 
with $\mathrm{D}=1000 \mathrm{~ms}$, and 8 Cloning degrees. ............. 16

Figure C-27: The Throughput of the H-ORB, the F-ORB and the P-ORB with

$\mathrm{D}=1000 \mathrm{~ms}$, and 8 Cloning degrees. .............. 16

Figure C-28: Cpu \& Process Utilizations of the F-ORB with $D=1000 \mathrm{~ms}$, and 8 Cloning degrees. . . . . . . . .

Figure C-29: Cpu \& Process Utilizations of the P-ORB with D $=1000 \mathrm{~ms}$, and 8 Cloning degrees. . . . . . . .

Figure D-1: The Response Time of the H-ORB, the F-ORB and the A-ORB with Equidistant Nodes, and $\mathrm{D}=1000$ ms. . . . . . . . . . . D-2

Figure D-2: The Throughput of the H-ORB, the F-ORB and the A-ORB with Equidistant Nodes, and $D=1000$ ms. . . . . . . . . . . D-2

Figure D-3: The Process and Cpu Utilizations of the A-ORB with Equidistant Nodes, and $\mathrm{D}=1000 \mathrm{~ms} . \ldots \ldots \ldots \ldots \ldots \ldots \ldots \ldots \ldots \ldots$

Figure D-4: The Process and Cpu Utilizations of the A-ORB with Equidistant Nodes, and $D=1000$ ms. . . . . . . . . . . D-3

Figure D-5: The Process Utilization of the A-ORB with Equidistant Nodes,

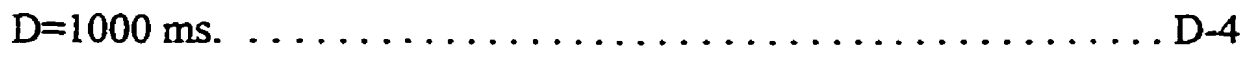




\section{List of Tables}

Table 1: Levels for the Workload Factors. ..................... 62

Table 2: Parameters of the Local H-ORB Experiments . . . . . . . . . 88

Table 3: Cpu and Elapsed Time of the Invoked Functions in a Client Request Cycle in a Local H-ORB Environment. . . . . . . . . . 89

Table 4: Parameters of Message Size Experiments ............... 91

Table 5: Effect of $L$ on Latency at $N=1\left(D=200 \mathrm{~ms}, S_{A} / S_{B}=10 \mathrm{~ms} / 15 \mathrm{~ms}\right.$, no Cloning) . . . . . . . . . . . . . .

Table 6: Throughput at $\mathrm{N}=24\left(\mathrm{D}=200 \mathrm{~ms}, \mathrm{~S}_{\mathrm{A}} / \mathrm{S}_{\mathrm{B}}=10 \mathrm{~ms} / 15 \mathrm{~ms}\right.$, no Cloning) $\ldots \ldots \ldots \ldots \ldots \ldots \ldots \ldots \ldots \ldots \ldots \ldots \ldots \ldots$

Table 7: Effect of $L$ on Latency at $N=1\left(D=200 \mathrm{~ms}, S_{A} / S_{B}=10 \mathrm{~ms} / 15 \mathrm{~ms}\right.$,

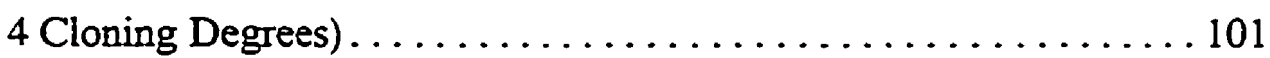

Table 8: Throughput at $N=24\left(D=200 \mathrm{~ms}, \mathrm{~S}_{\mathrm{A}} / \mathrm{S}_{\mathrm{B}}=10 \mathrm{~ms} / 15 \mathrm{~ms}\right.$,

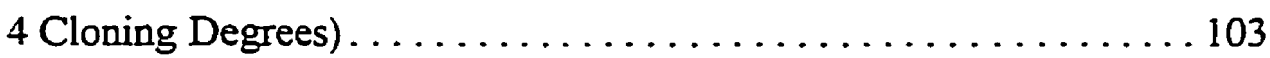

Table 9: Effect of $L$ on Latency at $N=1\left(D=200 \mathrm{~ms}, S_{A} / S_{B}=10 \mathrm{~ms} / 15 \mathrm{~ms}\right.$,

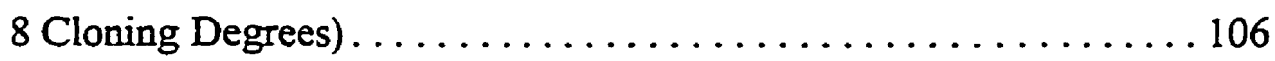

Table 10: Throughput at $\mathrm{N}=24\left(\mathrm{D}=200 \mathrm{~ms}, \mathrm{~S}_{\mathrm{A}} / \mathrm{S}_{\mathrm{B}}=10 \mathrm{~ms} / 15 \mathrm{~ms}\right.$, 8 Cloning Degrees) ............................. 107

Table 11: Parameters of Server Demand Experiments .............. 108

Table 12: Effect of $S_{A} / S_{B}$ on Latency at $N=1(D=10 \mathrm{~ms}, L=150$ Bytes, no Cloning) ............................... 109

Table 13: Throughput at $\mathrm{N}=24$ ( $\mathrm{D}=10 \mathrm{~ms}, \mathrm{~L}=150$ Bytes, no Cloning) $\ldots \ldots 113$

Table 14: Effect of $S_{A} / S_{B}$ on Latency at $N=1(D=10 \mathrm{~ms}, L=150$ Bytes,

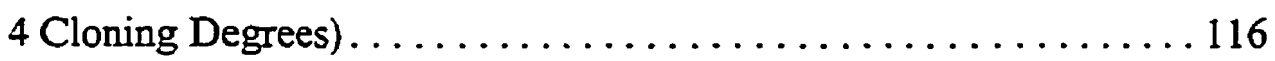


Table 15: Throughput at $\mathrm{N}=24(\mathrm{D}=10 \mathrm{~ms}, \mathrm{~L}=150$ Bytes, 4 Cloning Degrees) . . . . . . . . . . . . . . . . 117

Table 16: Parameters of the Inter-Node Delay Experiments . . . . . . . . 121

Table 17: Effect of $D$ on Latency at $N=1\left(L=150\right.$ bytes, $S_{A} / S_{B}=10 / 15 \mathrm{~ms}$, no Cloning) . . . . . . . . . . . . . . . . 122

Table 18: Throughput at $\mathrm{N}=24\left(\mathrm{~L}=150\right.$ bytes, $\mathrm{S}_{\mathrm{A}} / \mathrm{S}_{\mathrm{B}}=10 / 15 \mathrm{~ms}$,

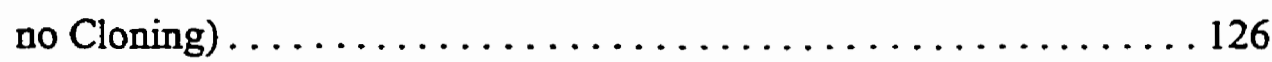

Table 19: Effect of $D$ on Latency at $N=1\left(L=150\right.$ bytes, $S_{A} / S_{B}=10 / 15 \mathrm{~ms}$, 4 Cloning Degrees) $\ldots \ldots \ldots \ldots \ldots \ldots \ldots \ldots \ldots \ldots \ldots \ldots \ldots \ldots$

Table 20: Throughput at $\mathrm{N}=24\left(\mathrm{~L}=150\right.$ bytes, $\mathrm{S}_{\mathrm{A}} / \mathrm{S}_{\mathrm{B}}=10 / 15 \mathrm{~ms}$, 4 Cloning Degrees) . . . . . . . . . . . . . . . . . . . 127

Table 21: Effect of $D$ on Latency at $N=1\left(L=150\right.$ bytes, $S_{A} / S_{B}=10 / 15 \mathrm{~ms}$,

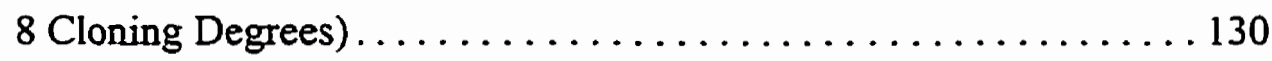

Table 22: Throughput at $\mathrm{N}=24\left(\mathrm{~L}=150\right.$ bytes, $\mathrm{S}_{\mathrm{A}} / \mathrm{S}_{\mathrm{B}}=10 / 15 \mathrm{~ms}, 8$ Cloning

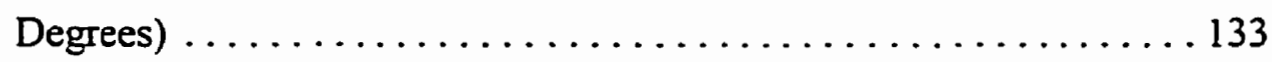

Table 23: Levels for the A-ORB Workload Factors . . . . . . . . . . . . 148

Table 24: Parameters of the A-ORB Inter-Node Delay Experiments. . . . . . 156 


\section{Glossary of Terms}

$\begin{array}{ll}\text { A-ORB } & \text { Adaptive ORB } \\ \text { BOA } & \text { Basic Object Adapter } \\ \text { CDS } & \text { Cell Directory Service } \\ \text { CORBA } & \text { Common Object Request Broker Architecture } \\ \text { DCE } & \text { Distributed Computing Environment } \\ \text { DFS } & \text { Distributed File Service } \\ \text { DII } & \text { Dynamic Invocation Interface } \\ \text { DOC } & \text { Distributed Object Computing } \\ \text { DSI } & \text { Dynamic Skeleton Interface } \\ \text { F-ORB } & \text { Forwarding ORB } \\ \text { H-ORB } & \text { Handle-Driven ORB } \\ \text { IDL } & \text { Interface Definition Language } \\ \text { IIOP } & \text { Internet Inter-ORB Protocol } \\ \text { IPC } & \text { Interprocess Communication } \\ \text { OMA } & \text { Object Management Architecture } \\ \text { OMG } & \text { Object Management Group } \\ \text { ORB } & \text { Object Request Broker } \\ \text { OSF } & \text { Open Software Foundation } \\ & \\ \text { Orav }\end{array}$




\section{P-ORB Process Planner}

RPC Remote Procedure Call 


\section{Chapter 1 Introduction}

\subsection{An Overview of Distributed Systems}

The computer industry has been going through a continuous evolution over the past few decades. In the mid -1980 , however, the evolution line experienced a major leap in the process of development due to two major advances in the computer technology, namely, the fabrication of powerful microprocessors and the invention of high-speed computer networks [TAN-95]. These improvements have significantly contributed towards the feasibility and ease of implementing computer systems composed of large numbers of processors (CPU's) connected over high-speed network(s). These systems are called the Distributed Systems in contrast to their ancestors, the Centralized Systems. Hence a Distributed System can be generally defined as: $A$ group of physically separated and independent computer systems connected over a network and giving their users the impression of a single powerful system.

Computer experts agree that distributed computing has many advantages over centralized computing including: reliability, performance, scalability, extensibility, and cost effectiveness. However, implementing such systems is a major task due to the limitation of the available conventional design tools and techniques such as BSD sockets and Sun RPC's, and the tendency to use functional decomposition techniques leading to statically configured systems [SCH-95.1]. The following section introduces one of the major computing trends of this decade, the Distributed Object Computing approach. 


\subsection{Distributed Object Computing (DOC)}

In an object-oriented environment, method invocation on a object is similar to the way the majority of distributed software system communicate (e.g RPC, bytestream communication, etc.). The decentralized nature of distributed systems gives a strong motivation towards adopting the object-oriented approach when implementing modern distributed systems, as well as the fact that Object Orientation can be highly effective in offering most of the distributed system features (e.g. data hiding, code reusing, portability) to distributed applications and thereby realizing the so-called Distributed Object Computing System [SCH-95.1].

The most important characteristics of these distributed object computing systems include:

a Providing object-oriented features to improve procedural RPC toolkits.

b - The interaction between distributed applications can be realized at a higher level of abstraction.

c. Providing the means for better coordination and collaboration among distributed applications at yet a higher level of abstraction leading to the realization of the socalled "Software IC's". Thus, the various object vendors will be able to market their software components which provide diversified implementations of industrystandard interfaces. As a result, the users will be able to select the suitable blend of those components that meets their design goals in an efficient manner. 


\subsection{Motivation for the Thesis}

In today's world, networked computing environments are characterized by a huge diversity in hardware and software. Hence, the need for interoperability among those heterogeneous systems is rapidly increasing. The notion of middleware has been introduced to provide interoperability as well as transparent location of servers in heterogeneous distributed client-server environments. The Common Object Request Broker Architectures (CORBA), proposed by the Object Management Group (OMG), is one of the commonly used standards for middleware architectures. CORBA, which is based on DOC, offers the advantage of code reuse since it combines the concepts of distributed computing with object oriented computing. In order to achieve a high performance middleware software, the system architecture should be carefully considered. This thesis investigates the relative performance of four different CORBA-based client-server architectures, the Handle driven ORB (H-ORB), the Forwarding ORB (F-ORB), the Process Planner (P-ORB), and the Adaptive ORB (A-ORB). The research focuses on studying the impact of these clientserver architectures on system performance. To the best of our knowledge, this problem has not yet been addressed by other researchers. Much of the earlier work has been concerned with the various techniques in the development of CORBA client applications and the performance of specific server implementations.

\subsection{Goals of the Thesis}

The primary goal of this thesis is to design, implement, and investigate the relative performance of different CORBA-based client-server architectures. Based on a 
commercially available CORBA compliant ORB software called ORBeline, three different architectures described in the literature [ADL-95] were designed and implemented, the $\mathrm{H}$ ORB, the F-ORB and the P-ORB. In the H-ORB, the client gets the address of the server from the agent and communicates with the server directly. In the F-ORB, the client request is automatically forwarded by the agent to the appropriate server which then returns the results of the computations to the client. In the P-ORB, the agent combines request forwarding with concurrent invocation of multiple servers for complex requests that require services of multiple servers. The relative performance of these architectures was investigated under different work load conditions. This thesis addresses the following questions:

i) Do software/hardware bottlenecks exist in the various architectures?

ii) What is the effect of the size of the message exchanged between the client and the server on system performance?

iii) What is the impact of the inter-node delays on system performance?

iv) How does agent cloning affect the performance of the F-ORB and the P-ORB? Is cloning beneficial over all values of workload parameters?

v) How sensitive is each architecture to the increase in the workload parameters?

Another important contribution of the thesis is the investigation of a new ORB architecture called the Adaptive ORB (A-ORB). The A-ORB combines the functionalities of both the H-ORB and the F-ORB and can switch dynamically from an H-ORB mode to an F-ORB mode and vice versa depending on the load condition. 


\subsection{Thesis Outline}

This thesis is composed of seven chapters. Chapter 2 discusses DOC systems design and analysis, and gives an overview of the various development techniques used in building distributed applications. Overviews of CORBA and Distributed Computing Environment (DCE) are presented in Chapter 3 along with a comparison between the two approaches. Chapter 4 describes the steps needed to develop CORBA applications and provides a highlevel description of the H-ORB, the F-ORB and the P-ORB as well as their respective execution scenarios. Comparison of system performance exhibited by these architectures under various workload and environment conditions is covered in Chapter 5. Chapter 6 introduces the A-ORB along with its respective experimental results and analysis. Conclusions and recommendations for furure research are presented in Chapter 7. 


\section{Chapter 2 Background}

\subsection{DOC System Design and Analysis}

A Distributed system attempts to disperse its services over networked computer systems using the proper implementation strategies to harvest the most out of the benefits of distributed environments such as efficiency, robustness, extensibility, and others.

The first step in designing a distributed system should be an in-depth analysis of the Environment Characteristics. To analyze a distributed environment, the following aspects are investigated:

a - The usual way of realizing a distributed system is to have the clients and the servers distributed over a set of independent computers and joined by different types of local and wide area networks (LANS \& WANS). Therefore, an important aim of DOC frameworks is to relieve application developers from the complexities of harmonizing the incompatible protocol-level details and to increase the portability and interoperability features.

b - The clients and servers may be installed on heterogeneous computers which may involve incompatible hardware platforms and could run on different operating systems. The code generated by DOC Frameworks' Interface Definition Language (IDL) and IDL compilers includes marshalling/unmarshalling routines for the remote method parameters. These marshalling/unmarshalling routines are responsible for encoding/decoding, respectively, binary data (for example, CORBA's representation 
of the parameters types and values) into/from a format that is recognizable throughout a heterogeneous system of computers with incompatible byte-order instruction sets. However, current frameworks vary in the extent of providing the applications with a portable interface for heterogeneous operating systems [SCH-95.2].

The second step in the analysis process is to study the Requirements of such distributed systems [SCH-95.2], including:

a Reliability: Detecting a failure in a distributed environment is more complex than that in a centralized environment.Therefore, in practice, distributed systems should offer a high level of reliability represented by the ability to distinguish between congestion delays and partial system failures; thereby offering a good degree of fault tolerance.

b - Availability: A large fraction of time the system is usable indicates high availability. Using replicas of Objects and services is one approach of achieving one component of that goal. Another component can be achieved through automatic crash recovery (done in collaboration with the first approach) and requires invoking the application under a continuous supervision. An example is Postmodern's (now known as Visigenic) ORBeline product where the Smart Agent tracks all processes partaking in a communication by sending periodic heartbeats, and reactivates the crashed object or reroutes further messages to the object's replica in case of detecting an object failure [ORB-94.1].

c- Object Location and Binding Flexibility: The Object Request Broker (ORB) has 
been developed to enable the clients to locate and communicate with the sought remote server using high-level names instead of the conventional transport layer interfaces such as BSD sockets. Service Traders on the other hand offer more flexibility to the client by locating the server that provides both the ability of meeting the client request as well as complying with the desired quality of service. An example case is when a client's printing request (requiring a laser quality printout as urgent as possible) has been sent to a pool of printers. It is the Trader's responsibility to identify the subgroup of servers offering laser quality printouts and route the request to the server with the shortest queue length and thereby realizing load balancing abilities.

d Performance: System throughput and hence the overall performance can be improved by several methods such as multithreading the servers, implementing asynchronous communication between the client and the server, thereby giving more chance to the client to perform other activities rather than blocking itself waiting for the reply. Another approach that helps improve performance is adopting the so-called Deferred Binding which utilizes the system resources more efficiently by locating the object at the start but activating it only on demand (i.e. at method invocations only).

\subsection{Development Techniques for Distributed Applications}

There are several challenging tasks that have to be overcome by software developers in order to obtain robust, efficient and extensible distributed applications [SCH-92.11]. Such 
tasks include a good knowledge of Networks Addressing, Presentation Conversion, Process and thread creation and synchronization, OS calls and InterProcess Communication (IPC) mechanisms.

An overview of the various programming techniques used in developing (writing) distributed applications is covered in the coming subsections starting with a lower-level approach followed by the higher levels.

Figure 2-1 shows the various IPC mechanisms used in modern operating systems at the various levels of abstractions [SCH-92.11].

\subsubsection{Lower Level Programming Interfaces}

Lower-level IPC mechanisms provide an interface to the operating system kernel's communication subsystems. An example of such mechanisms are the SVR4 system calls open, close, putmsg, and getmsg. Network services, such as network file systems can be developed using these mechanisms. In addition to the complexities of lower-level operating system calls, the major disadvantage is that such products are usually not portable not only across different operating systems platforms but also often among different versions of the same operating system since they are tightly tailored to the underlying kemel.

\subsubsection{Transport Layer Interfaces}

BSD Unix Sockets and System V Transport Layer Interfaces (TLI's) are examples of interprocess communication mechanisms (ipcs) that support a generic interface to multiple communication domains. 
User Space

High level of

User Space

Abstraction

RPC Toolkits (CORBA, DCE,.......)

$\mathrm{C}++$ Wrappers (IPC SAP,......)

Transport Layer Interfaces (BSD Sockets, System V TLI's....)

Lower Lever Programming Interfaces (Open/Close......)

Kernel Communication Subsystem

Kernel Space

Low level of

Abstraction

\section{Figure 2-1: IPC Interfaces in Modern OS Platforms}

It is more challenging to use the Transport Layer Interfaces when writing distributed applications, but it provides several advantages including:

- The omission of unnecessary functionality.

- Providing fine-grain control over functionality

- The ability to port the applications across a wider range of heterogeneous OS platforms than do RPC Toolkits especially for stream applications which can not tolerate the overhead of RPC's for many reasons including non-optimized presentation format conversion and memory management [SCH-92-11].

A short description of a transport layer interface, the Socket, is presented. The socket 
solution requires the programmer to pay careful attention to the following aspects throughout the development process:

1) A socket is bound to the address of the server since it represents an endpoint of communication. Hence, socket-based solutions will create an unfriendly and error-prone user interface since it requires the user to enter the actual network address. A slightly better solution is to allow the user to identify the servers by name (e.g. host name) and throw an extra burden on the programmer to write the additional code needed to derive the actual network address from the supplied host name, thereby creating some sort of a primitive Naming Server.

2) Socket programming requires a lot of detailed programming including Local Context Management, Connection Establishment and Termination, Data Transfer Mechanisms and Option Management, all of which need a tremendous efforts on the part of the programmer.

3) Moreover, the socket-based source code will not be portable if transferred to a non-socket based platform (e.g. System V), which will invariably entails major modifications to retain the same functionality.

\subsubsection{C++ Wrappers Interfaces}

$\mathrm{C}++$ wrappers were developed to alleviate the complexity of distributed application programming by encapsulating lower-level network interfaces within a type-safe objectoriented interface. The Inter-Process Communication Service Access Point (IPC SAP) [SCH-92.11] is an InterProcess Communications (ipc) class library which can be used 


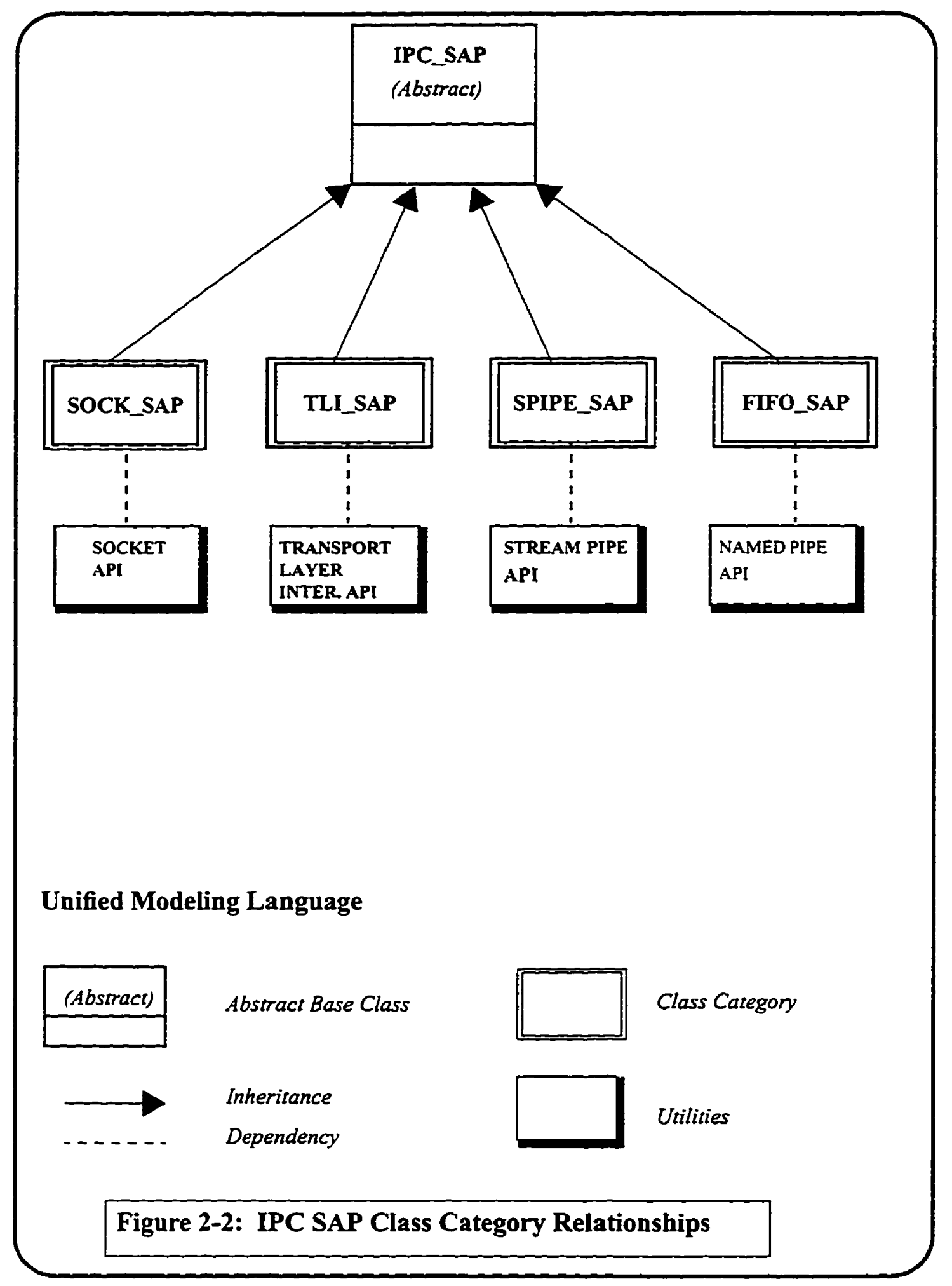


effectively to develop these $\mathrm{C}++$ wrappers. As shown in Figure 2-2, this library includes a group of class categories such as the SOCK SAP which encapsulates the socket interface.Each class category is organized as an inheritance hierarchy and provides an interface to a subset representing the existing lower-level IPC's. These sub-classes will collaborate to realize the so-called Inter-Domain protocol with a high degree of modularity and code reusing due to the heavy use of inheritance and parameterized types. Therefore, similarities between category classes are represented by the base classes while the subclasses are used to express the differences.

The IPC SAP is designed to improve correctness by making all the classes strongly typed thereby invalid operations will be rejected at compile time rather than at run time. Hence, all the inherent weak type-checking problems with sockets will be avoided.

Moreover, IPC SAP simplifies network programming interface by eliminating several common programming pitfalls through auxiliary classes that allow programmers to circumvent error-prone details. Ease of learning and use is attained by code reusing through the inheritance based hierarchal decomposition and portability by having a platform independent interface as in Figure 2-3 where a subset of the SOCK SAP and TLI SAP offer the same parameterized object-oriented interface. The fact that a majority of modern operating systems (such as the Solaris 2.5 ) can support both Sockets and TLI mechanisms justifies the importance of the IPC SAP-like wrappers. The heavy implementation of inline functions and abandoning the use of virtual functions help reduce the overhead of invoking the IPC SAP methods. 


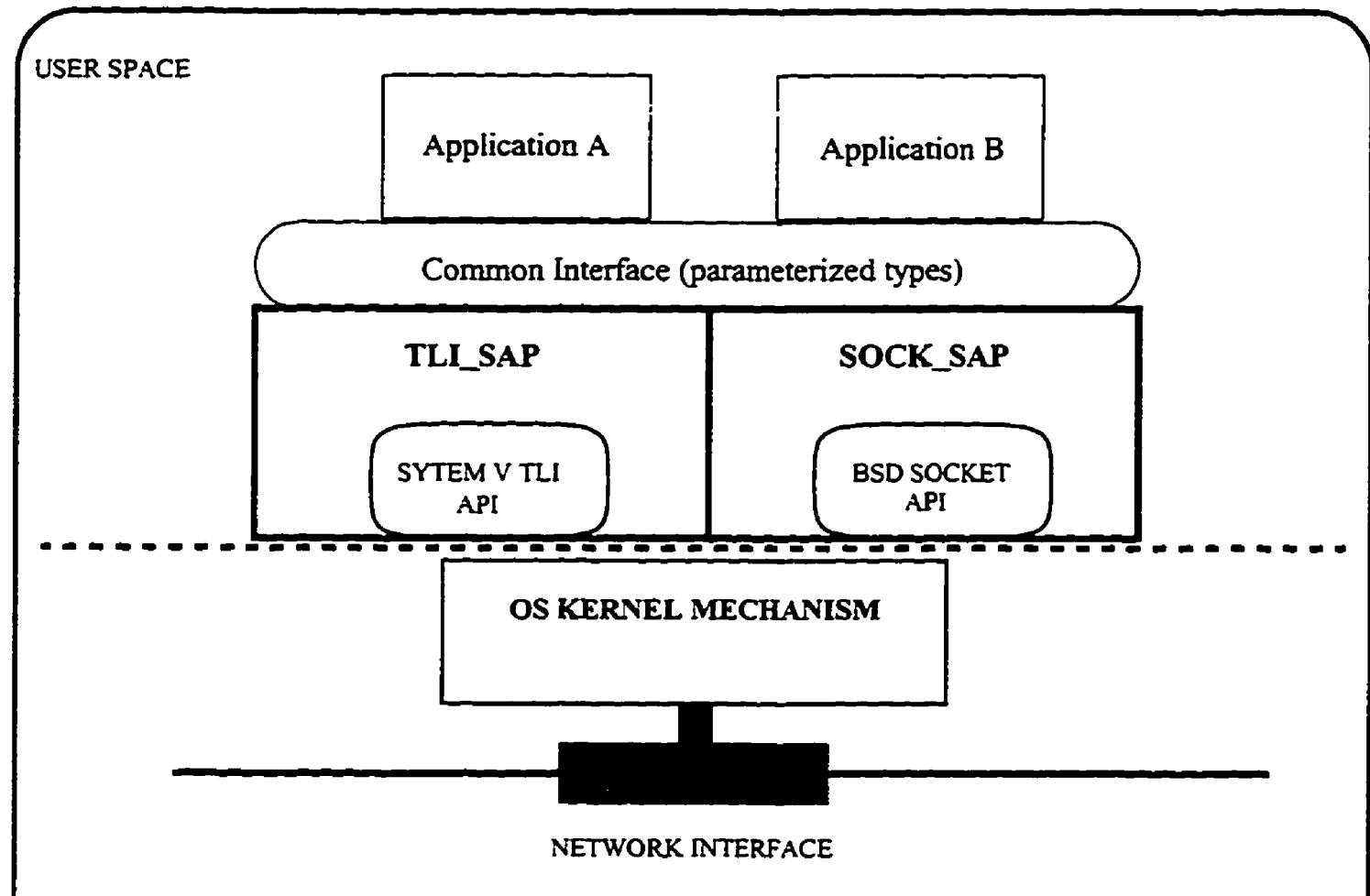

KERNEL, SPACE

Figure 2-3: The IPC SAP C++ Wrapper

A final word on the $\mathrm{C}+$ wrapper approach is that without a $\mathrm{C}++$ wrapper library like the IPC SAP developing an object-oriented communication infrastructure is a major task which will invariably divert the attention of the developers from the application details towards the network details. Also, should the requirements change, or the application being moved to a new different platform, major changes have to be performed. 


\subsubsection{RPC Toolkit Interfaces}

The next chapter gives a description of the OMG CORBA, and the Open Software Foundation's Distributed Computing Environment (OSF DCE) as RPC Toolkits used in the development of DOC systems. An evaluation and comparison of the two approaches are also presented. 


\section{Chapter 3 CORBA Overview}

\subsection{Introduction}

The Object Management Group (OMG) was formed in 1989 with the purpose of creating standards for the interoperability and portability of distributed object-oriented software applications. The OMG Object Management Architecture (OMA) defines at a higher level of abstraction the facilities necessary for distributed 00 computing.

Programming languages such as $\mathrm{C}++$ and Java have recently taken the lead in the development of distributed object computing systems. However, many programmers are confronted with a major problem:

- Object Migration: How would an application developer ship a C + object, for example, across a network from one process to another provided that the principles of information hiding and encapsulation are preserved?

The hardest problem with $\mathrm{C}++$ object migration is keeping each separate executable component of a distributed system synchronized with respect to different versions of the $\mathrm{C}++$ class types involved since it is difficult to accertain that each component has been compiled with the same declaration for each class type [VIN-93]. Systems like the Distributed Computing Environment (DCE) have RPC mechanisms for transmitting complex data structures but fail short in supporting $\mathrm{C}++$ objects, and the fact the $\mathrm{C}++$ objects contain hidden pointers to virtual functions aggravates the situation even more.

The importance of object migration stems from the fact that the majority of the existing 
client/server applications written in $\mathrm{C}++$ have evolved from their $\mathrm{C}$-written ancestors which involved sending $C$ structures across the network and hence it becomes necessary to have the ability to send them as full-fledged objects once the application is upgraded to $\mathrm{C}++$ code [VIN-93].

Therefore, Distributed $O O$ applications must deal with the object interfaces only which entails an Object Model that allows applications to transparently utilize both local and remote objects without degrading efficiency. Moreover, this model must be capable of providing a standard object programming interface that is both system-independent and language-independent.

\subsection{The CORBA Object Model}

The Object Model provides an organized presentation of object concepts and terminology. It defines a partial model for computation that embodies the key characteristics of objects as realized by the submitted technologies.

An Object System is a collection of objects that isolates the requestors of services (clients) from the providers of services by a well-defined encapsulating interface. Hence, clients are isolated from the implementations of services as data representations and executable code [OMG-95].

The core of the OMA is the Object Request Broker (ORB), a mechanism that provides transparency of object location, activation, and includes all the communication infrastructure needed to deliver requests and their associated parameters to the designated objects implementations (servers) and to return the results back to the requesting clients. 


\subsection{The Common Object Request Broker Architecture (CORBA)}

The OMG published the latest version (V-2.0) of the CORBA specifications in 1995, a concrete description of the interfaces and services that must be provided by compliant ORBs.

Figure 3-1 shows the structure of the Object Request Broker (ORB) along with its interfaces. Although its name suggests a single process, an ORB is in fact the embodiment of multiple system entities, including all service location facilities, data marshalling/ unmarshalling procedures, in addition to all the infrastructure needed to implement the CORBA standard.

CORBA is composed of the following major components

- ORB Core.

- Interface Definition Language (IDL).

i) IDL Stubs

ii) Static IDL Skeleton.

- Dynamic Invocation Interface.

- Dynamic Skeleton Interface.

- Interface Repository(IR).

- Object Adapters (OA). 


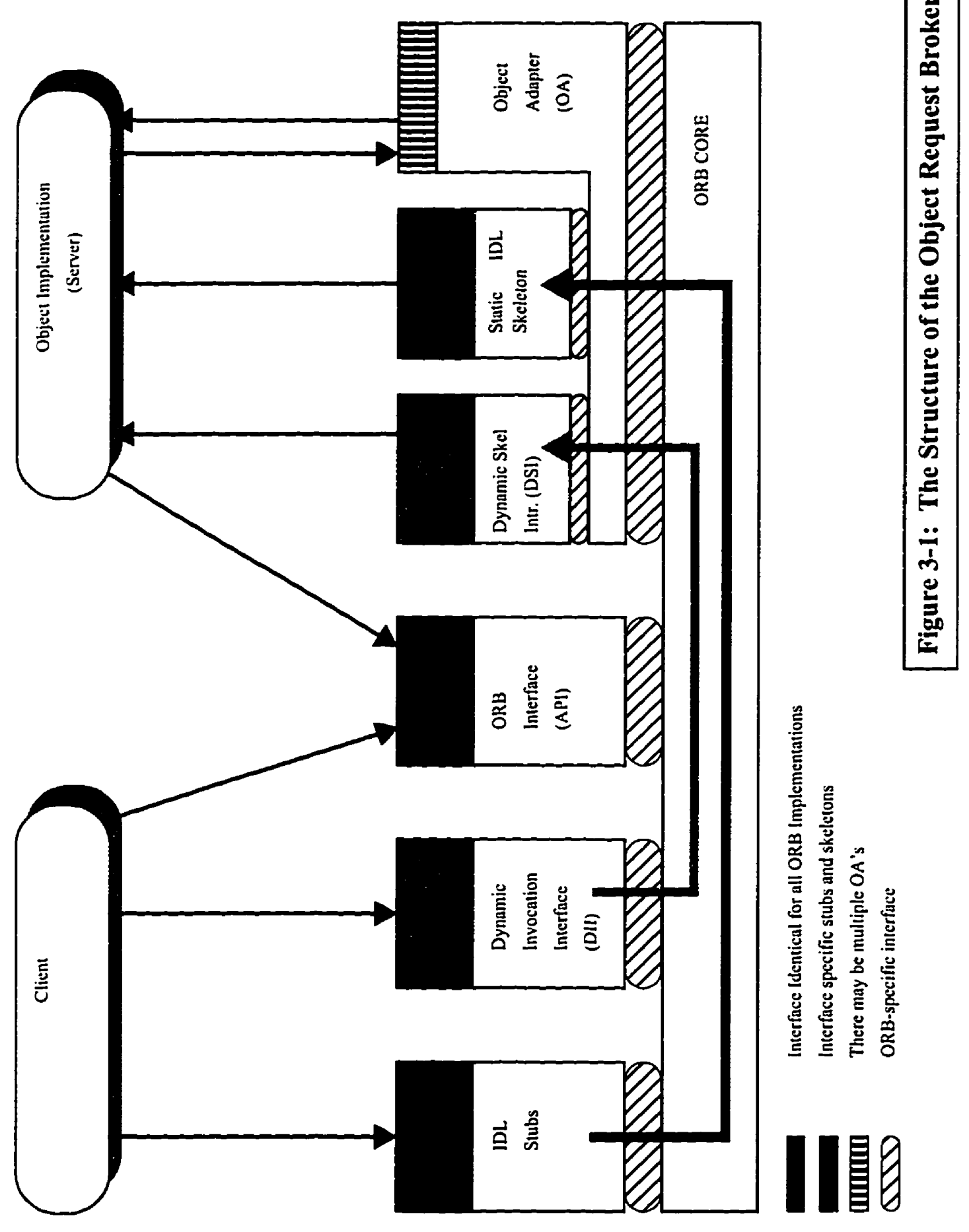




\subsubsection{ORB Core}

The ORB Core is the communication infrastructure responsible for locating desired objects, activating them if they are not executing, handling the connection management between processes and transmitting data associated with requests and responses between clients and server processes. Theses ORB services are completely transparent to the client. Clients do not need to know nor care about the location on the network where the sought objects reside, how they communicate, how they are stored, how they are implemented, nor how they execute. CORBA is designed to support different object mechanisms, and it does so by structuring the ORB with components above the ORB Core, that provide interfaces that can mask the differences between ORB Cores.

\subsubsection{The ORB Interface}

The ORB Interface is the interface to those ORB services that do not depend on which Object Adapter is used. These services are the same for all ORBs and all object implementations, and can be invoked by either the clients or the object implementations (servers). Some of these generic services include those used by the ORB to stringify (convert to a string format), duplicate, release and check the validity of object references. Moreover, this interface defines operations for creating lists and determining the default context used in the Dynamic Invocation Interface.

\subsubsection{CORBA Interface Definition Language (IDL)}

The OMG Interface Definition Language (IDL), a declarative language with a syntax resembling that of $\mathrm{CH}$, is the language used to describe the interfaces that clients of 
objects invoke and object implementations provide. The main characteristics of the IDL language are as follows:

a IDL provides basic data types, constructed types, and template types (such as a sequence).

b - Modules can also be defined. Similar to the C+t namespace, modules can be reopened to add more declarations to them.

c - IDL declares operations that uses the data types in (a) as arguments types and return types.

d - The interfaces declared by the IDL use the operations in (c) to define the services provided by the object implementations.

e IDL provides specific interface inheritance rules in which derived interfaces inherit the operations and types defined in their base interfaces. Because there is no concept of state in IDL, only behavior, a base interface can only be inherited once.

f - Exceptions can also be defined. CORBA defines a set of standard system exceptions, and users can define their own exceptions as well.

Clients are not written in OMG IDL but in languages for which mappings from OMG IDL concepts have been defined. Object implementations (servers) can utilize any of the inheritance features of their implementation language independent of the predefined IDL inheritance [VIN-93]. Figure 3-2 gives an example of an IDL source code with the interface declaration and its operations. 


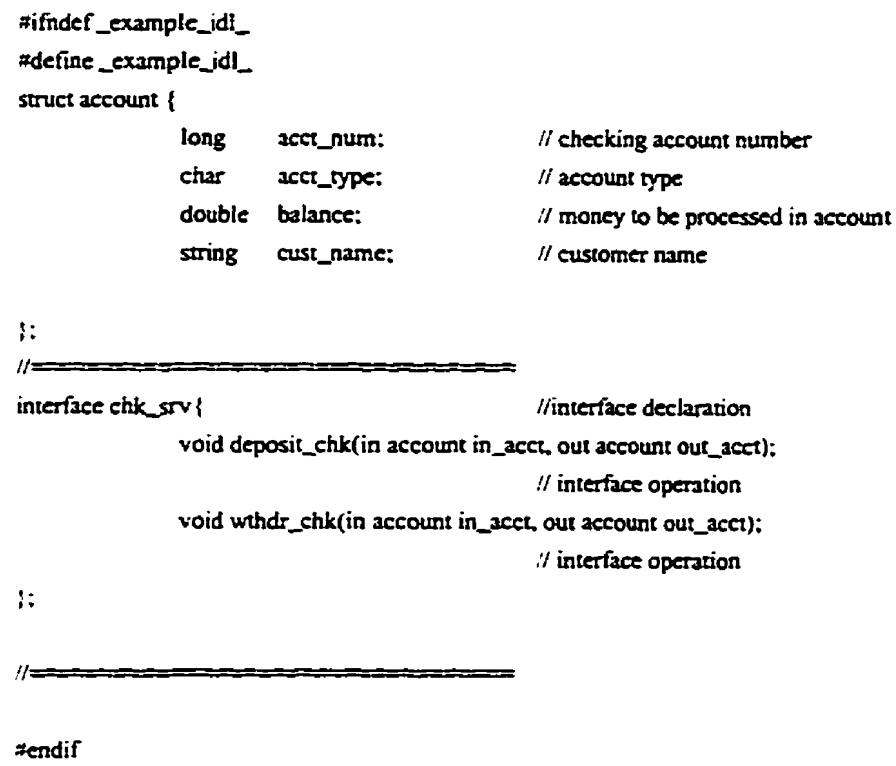

Figure 3-2: An example of a CORBA IDL source code

\subsubsection{The IDL Stubs}

The IDL stubs are generated automatically after compiling the IDL source code. Each stub provides access to one of the IDL declared operations on a CORBA object. The stubs make calls on the rest of the ORB using interfaces that are private to the underlying ORB core. Depending on the operation declaration in the IDL source code, each stub can have a marshalling/unmarshalling routine for the remote procedure parameters as well as exception handling mechanisms. Figure 3-3 has part of the generated C+ code (using ORBeline IDL compiler) for the IDL interfaces and operations in Figure 3-2 [ORB-94]. 


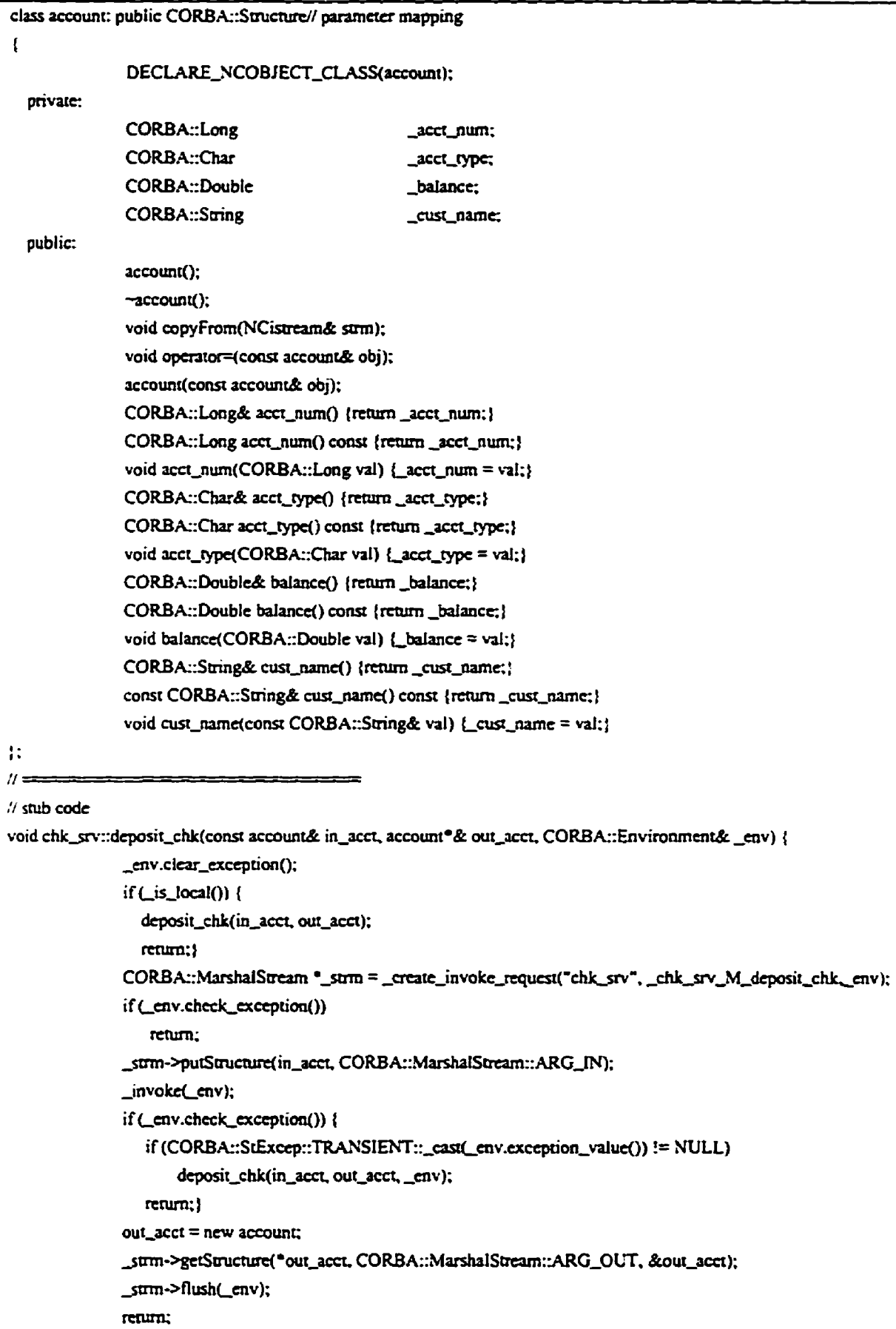

Figure 3-3: Partial C++ code generated by ORBeline IDL compiler for the source code of Figure 3-2 showing mapping of parameters and a Stub code. 
void chk_ssv_impl::_deposit_chk(void "obj, CORBA:-MarshalStream \&sstrm.

CORBA::Environment\&_env, CORBA::Principal "principal) )

chk_so__impl •_impl $=\left(\right.$ chk_sv_impl $\left.{ }^{\circ}\right)$ obj:

_env.ciear_exception0:

account in_acct:

strm.getStruchures in_acet.CORBA::MarshalStream::ARG_IN):

account" our_acer:

out_acet $=($ account" $)$ 0:

strm.Alush(env):

if (env.check_exception())

reaurn:

impl->_principal(principal):

_impl->deposit_chk(in_acct, out_acet):

strm.purEnvironment(env):

strm.purStructure(out_acet.CORBA::MarshalStream::ARG_OLT):

_impl->_principal((CORBA::Principal •) NULL):

:

Figure 3-4: One of the Static Skeletons generated by ORBeline IDL compiler for the source code of Figure 3-2.

\subsubsection{The Static DL Skeleton}

Another product of the IDL compilation process is the generation of a static skeleton for each operation declared in the original IDL source code. This skeleton is generally an up-call interface, in that the object implementation must develop routines that conform to the interface which will be called by the ORB core and the respective OA through the skeleton. Depending on the declared operation, each skeleton can have unmarshalling/ marshalling routine as well as exception handling mechanisms [OMG-95]. An example of a static skeleton (generated by ORBeline IDL compiler) is shown in Figure 3-4.

\subsubsection{Dynamic Invocation Interface (DII)}

The Dynamic Invocation Interface (DII) allows clients to invoke methods on an object 
implementation without having access to the object's stubs and without having an IDL compiler.The client code (request) must supply information about the operation to be performed and the types of parameters being passed (obtained from the Interface Repository or some other runtime source). Some DII implementations perform typechecking at runtime on the client-side to ensure that the values passed in at invocation time are correct [ORB-94].

Therefore, the DII is a generic client-side stub capable of forwarding any request to any object implementation (server) throughout runtime interpretation of request parameters and operation identifier. This DII flexibility is costly, however. A remote object invocation made through the stub/skeleton pair can be achieved in a single RPC, but the same call made through the DII would require a series of function calls including the overhead of creating the Request object, adding in the parameters and then invoking the dynamic request. For applications written in a compiled language such as $\mathrm{C}++$, it is far more efficient to issue requests via the IDL stub/skeleton pair than through DII [OMG-95][VIN-93].

\subsubsection{Dynamic Skeleton Interface (DSI)}

This interface permits the dynamic handling of the object (server) invocations. In other words, the DSI is used to implement all requests to all operations on a particular object by having the ORB invoke the same upcall routine. This generic interface that provides access to the operation name and parameters in a manner similar to the client's DII. Dynamic knowledge of these parameters, from the Interface Repository, can be used to determine them [OMG-95]. 


\subsubsection{Object Adapter (OA)}

The Object Adapters OA's are intended to adapt object implementations (servers) written in different styles and languages to the ORB. The ORB and the OA cooperate to ensure smooth and correct communication between the client and the respective server regardless of their respective languages.

The Object Adapter is the primary way that an object implementation accesses services provided by the ORB such as:

- Generation and interpretation of Object References.

- Method invocation.

- Object implementation activation and deactivation.

- Mapping of object references to implementations.

- Registration of implementation.

Based on the ORB implementation, the OA may provide these services by delegating to the ORB or by performing the task itself. In either way, the object implementation is unaware of who is actually doing the job since it interfaces only to the OA. CORBA has designed the Basic Object Adapter (BOA) to be the most commonly used object adapter. All CORBA compliant implementations must have a BOA as part of their ORBs [OTT96][SIE-96][OMG-95][VIN-93].

\subsection{CORBA, a Development Technique for DOC Systems}

As described above, the ORB supports Distributed Object Computing (DOC) in 
accordance with the OMG CORBA (V2.0) specifications.

Due to the ORB reusable infrastructure, application designers are relieved from many of the complex and error prone problems usually faced with the lower-level network interface programming [SCH-95.5]. As a short description of the service invocation, the client must obtain the sought object reference, also called the handle to the server object. The ORB core is responsible for automating all the underlying common communication activities, such as locating the server object, activating it (if need be), passing the client request to the server, and returning the response back to the client [OTT-96][SCH-95.2].

It can be concluded from the above discussion that a CORBA-based client development is done at a higher level of abstraction than the other two approaches, the Transport Layer and the C++ Wrapper Interfaces, discussed in Chapter 2; however, it may require the programmer to spend a good deal of time to master the specifications.

The Internet Inter ORB Protocol IIOP has been recently accepted by the OMG and has become mandatory for CORBA (V2.0) compliance [SIE-96]. The OMG, based on the response of the various vendors to its request for proposal, is continuously evaluating new features to be added to the CORBA specification, such as the Security specifications. It can be inferred from the growing markets for CORBA-based systems that such systems can become the common infrastructure for a wide range of applications [SIE-96].

The performance of this approach is not as good as the socket-based or the C++ wrappers-based applications due to the fact that the ORB is not tuned to any specific applications and obtaining the handle might require one or more remote invocations on its 
own. On the other hand, CORBA-based applications are more flexible to changes in the requirements than do sockets and $\mathrm{C}++$ wrappers. For example, in a non-DOC system, adding new features to the application might require passing extra parameters between the application and the server implementation which entails major modifications of the source code as well as the need to take the system down for an upgrade. In contrast, DOC frameworks such as CORBA can easily handle such changes in the requirements by adding the extra parameters to the IDL operations signature in the interface definition. However, such changes must be followed by recompiling both the client and server codes to accommodate such changes and probably shutting the system down. Therefore, a better approach will be using CORBA's interface inheritance feature.

To achieve this, a first step would be to create another interface with the additional features and then create a new interface that inherits from both interfaces (the old and the new). This approach allows old clients to be able to connect and use the new improved servers. However, new clients invoking a stub for the new server will not be able to connect to an old limited server. Moreover, a set of tools and standards are yet to be developed to manage and preserve the integrity and consistency of software components in an extensive DOC system.

\subsection{The Distributed Computing Environment (DCE)}

As mentioned earlier in Chapter 2, distributed applications require the existence of a distributed environment in which they run. Many distributed software vendors have created their proprietary environments for which they have implemented solutions to the classical 
problems such as authentication, authorization, and data integrity. However, these solutions are not portable across heterogeneous vendor's platforms [LOC-94]. The Open Software Foundation's (OSF) DCE provides a vendor-neutral platform for building distributed applications. DCE comprises a common set of support services for distributed applications. It builds on work already done by many vendors for their own environments.

The key issues addressed in DCE [CHA-96] are briefly discussed.

i) DCE uses remote procedure calls (RPC's) for communications between the components of a distributed application.

ii) DCE provides a threads package to support parallelism in distributed applications.

iii) DCE comprises several options for Directory Services. The Cell Directory Service (CDS) in used for LANS, while the Domain Name System (DNS) or X.500 can be used for Global Directory Service.

iv) To meet Security requirements, DCE provides authentication, authorization, data integrity, and data privacy.

v) DCE introduces a Distributed Time Service (DTS) for clock synchronization which can be integrated with the Internet Network Time Protocol (NTP).

vi) DCE includes only one distributed application, the Distributed File System (DFS).

Figure 3-5 shows the various components of DCE and the dependency relationships among them [CHA-96]. 


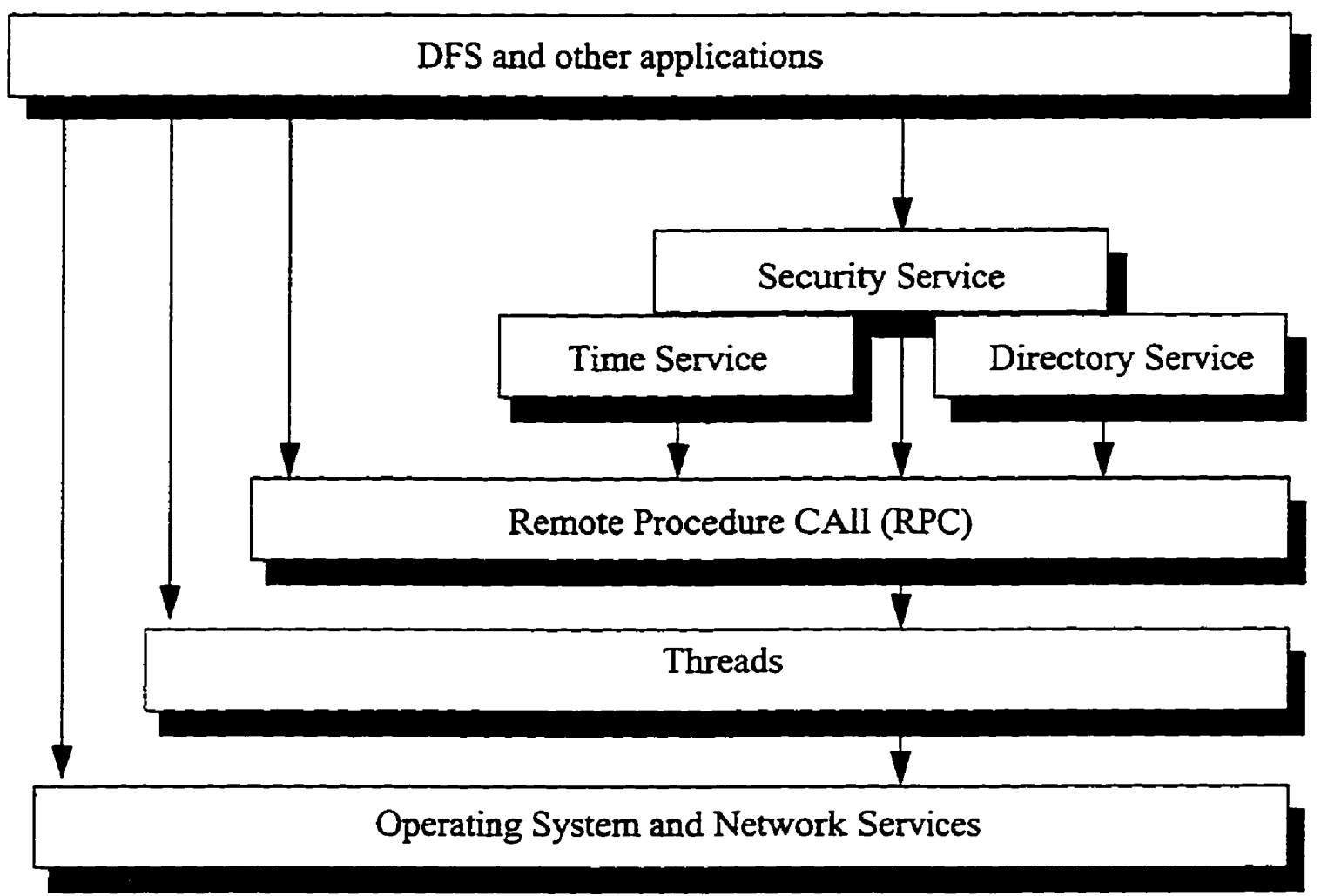

Figure 3-5: The DCE Components and their dependency relations

\subsection{CORBA and DCE}

A comparison between CORBA and DCE [CHA-96] is described briefly.

1) DCE provides an infrastructure for supporting distributed applications, including: an RPC mechanism, directory service, and distributed security. CORBA defines interfaces to an infrastructure for supporting distributed applications which includes: an RPC-like mechanism, directory service, and 
distributed security.

2) DCE is created and controlled by the OSF, whereas CORBA is controlled by the OMG and defined by consensus-oriented committees.

3) OSF provides a reference implementation of DCE which is used by most vendors. Therefore, portability of DCE applications across different vendor's platforms is relatively easy. Since there is no reference implementation of CORBA, each vendor has its own implementation. As a consequence, portability of CORBA applications across different vendor's products is possible but non-trivial [CHA-96].

4) A DCE server can support one or more interfaces while a CORBA sever can support only one interface. In DCE only synchronous calls are supported. Hence DCE clients and servers must use stubs for marshalling/unmarshalling. CORBA clients and servers may use stubs and skeletons for static invocations or DII for dynamic invocations. Therefore, synchronous, deferred synchronous, and one-way asynchronous mechanisms can be used for communication.

5) The DCE IDL language syntax is derived from the $C$ language. Each interface is assigned a UUID (Universal Unique Identifier) and a version number. Interfaces can not inherit from one another. On the other hand CORBA's IDL syntax is derived from the $\mathrm{C}+$ language. The interfaces do not have nor require any UUID's or version numbers, but can inherit from one another. 
6) A DCE client obtains the handle for a server from the Cell Directory Service. In CORBA, the client must obtain an object reference for the server either from the directory service provided by the agent or from other special objects. 


\section{Chapter 4 The Request Broker Architectures}

\subsection{Definitions}

Listed below, a group of definitions and explanations for the terms and keywords introduced in this chapter is presented.

\section{The Object}

An Object (also known in CORBA as the object implementation or the server) is an identifiable, encapsulated entity that provides one or more services that can be requested by a client.

\section{The Object Request Broker (ORB)}

An Object Request Broker ORB is a specialized mechanism that may be applied to structure DOC software systems with decoupled components by mediating the interactions between client applications requesting services and server applications (also known as object implementations) capable of providing these services. Hence, all server applications in such distributed systems must register statically/dynamically their names, the services they support (the corresponding client interfaces) in addition to their locations with the ORB. 


\section{The ORB core}

The ORB core, the communication infrastructure of the ORB, is responsible for coordinating the communication between clients and servers such as forwarding requests as well as transmitting results and exceptions.

\section{The ORB Agent (Broker)}

The Agent (broker) is a dynamic directory service that keeps track of all object implementation (servers) that are either currently active or are registered with their respective activation daemons and will be activated when invoked. Moreover, the Agent keeps track of all processes participating in a communication by sending periodic heartbeats, and when a connection is broken by accident then the Agent along with the other ORB components will cooperate to reestablish the connection between the client and the object or a replica of the object if available [ORB-94]. The X.500 Directory standard also addresses the issue of name resolution in distributed systems [SME-91]. However, the CORBA agent goes beyond the services offered by the X.500 by offering load balancing through equal distribution of requests on object replicas in addition to the previously discussed fault tolerance ability.

\section{The Object Reference}

An Object Reference is the information needed to identify an object within an ORB. Both clients and object implementations have an opaque notion of object references according to the language mapping, and thus are insulated form the actual representation of them. Therefore, an object reference uniquely identifies an object within an ORB 
domain, and is produced as a result of the creation of an ORB object. All ORBs must provide the same language mapping to an object reference for a particular programming language [OTT-96]. Consequently, a client written in a specific language can access object references independent of the particular ORB. A client must acquire the object reference of the sought object (server) before it can issue a request to it. As long as the object is executing (i.e. the object implementation is alive) its object reference is valid and the ORB allows the holder of that reference to request services from the referenced object. Since it is not convenient to directly store object references in persistent storage or to communicate them by means other than invocation, the clients must ask the ORB to convert them into strings which makes it possible to store them as private data for later needs [SIE-96].

\subsection{CORBA Applications Development Steps}

As mentioned in Chapter 2, there are many factors to consider when developing a practical distributed system, including scalability, resource utilization, extensibility. The steps listed below represent a general approach for the complete development phase of CORBA applications. It would be possible to use some of the steps only to develop new object implementations (to enhance the existing quality of service), or to write a new client application that benefits from the already available servers etc. It is also assumed that the applications are developed in the $\mathrm{C}++$ language. A description of the main approach is presented in the next subsection.

\subsubsection{The IDL mapping approach}

The OMG IDL to $\mathrm{C++}$ mapping and the subsequent steps are discussed in this 
subsection:

1) The first step is always the IDL definitions. Hence the object interfaces are designed and specified according to needed functionalities of the servers using the OMG IDL.

2) The IDL source code is compiled by the $\mathrm{DL}$ compiler to generate automatically the Client Stubs and Server Skeletons which serve as the glue between the client and the server implementations, respectively, on one side and the ORB core on the other side. The stubs and skeletons will be generated in a full-fledged $\mathrm{C}+$ language (as illustrated in Figure 4-1). Moreover, IDL interfaces are mapped to $\mathrm{C}++$ classes. Interface Operations are mapped to member functions of those classes and other data types are converted as specified. For example, an IDL String is mapped to a pointer to the first character (char*)and so on. A sample of the generated code is presented in Chapter 3 in Section 3.3.

3) CORBA objects are implemented as $\mathrm{C}+$ objects.

4) The Server (object implementation) main program is implemented.

5) The Client Application using the CORBA objects is implemented.

\subsubsection{External Installation}

The Object implementation information is provided at the installation time and is stored in the Implementation Repository which will be questioned during request delivery. The ORB provides routines for accessing the implementation repository to update/get the 


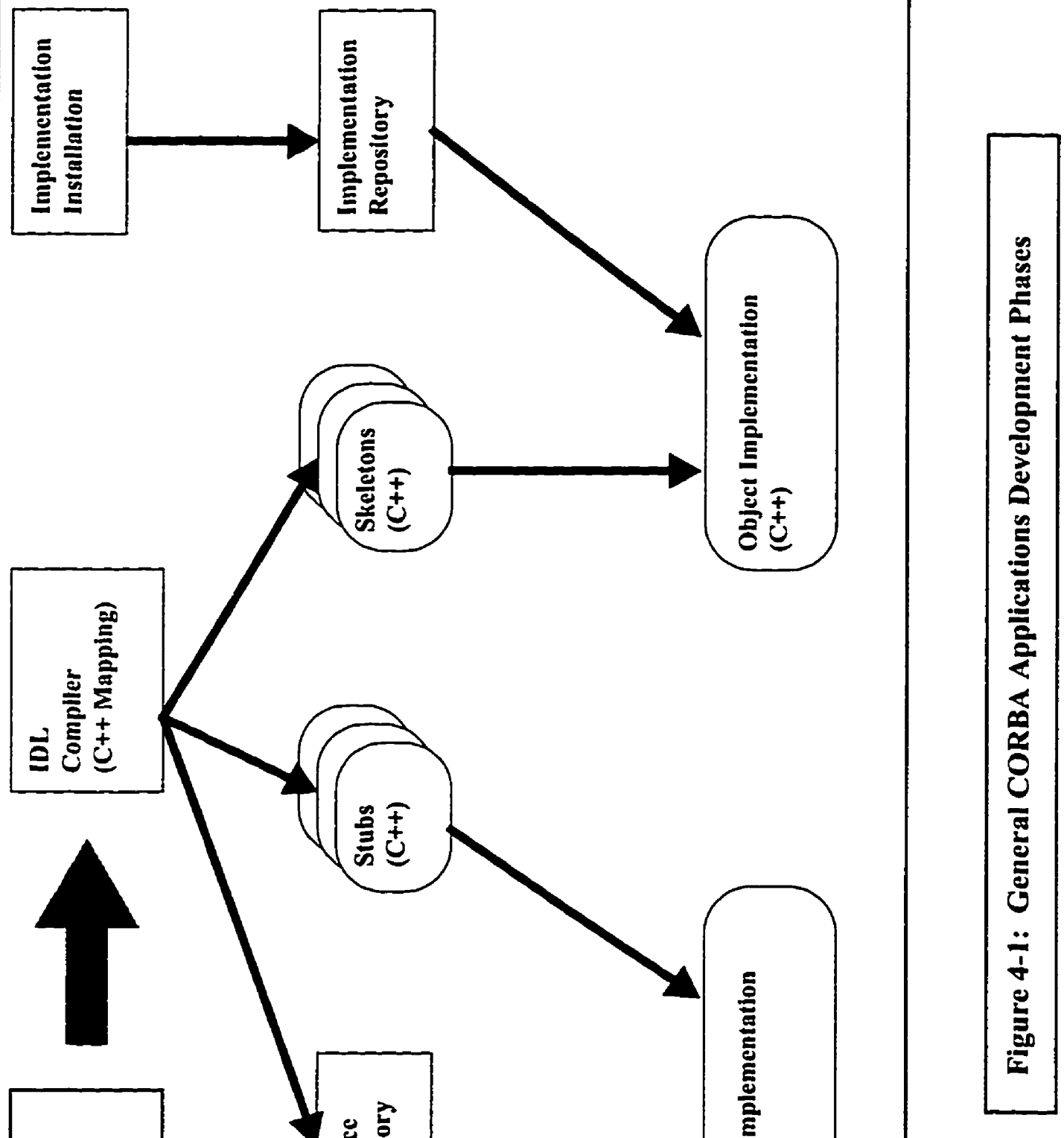


needed information on an object implementation.

The following sections give a detailed description of the default CORBA architecture (as provided by ORBeline), the Handle-Driven ORB, followed by two other CORBA architectures, the Forwarding ORB and the Process-Planner ORB. Each ORB behaves differently in terms of the steps followed by the client request to reach the desired server and by the reply to return to the originating client.

\subsection{The Handle-Driven ORB (H-ORB) Architecture}

This section presents the Handle-Driven ORB. The name was adopted from an earlier publication by Adler [ADL-95]. The Object Management Group's Common Object Request Broker Architecture (OMG CORBA) adopts this request-handling approach. The discussion covers a description of the main components of this architecture as well as its execution scenario.

\subsubsection{Definition}

The handle-driven ORB is an architecture in which the agent (broker) returns a service "handle" back to the requesting client. This handle, also called the Object Reference, is the means by which services are requested from a CORBA object (server) since it contains all the information required to interact with the server application (the object implementation) for the sought services. Therefore, the client can, using this handle, invoke the remote procedure on the object implementation, which in turn sends the reply (if needed) back to the client. Moreover, these Object References realize Location Transparency i.e. the client can not infer from the handle whether the sought object implementation is local (in the 


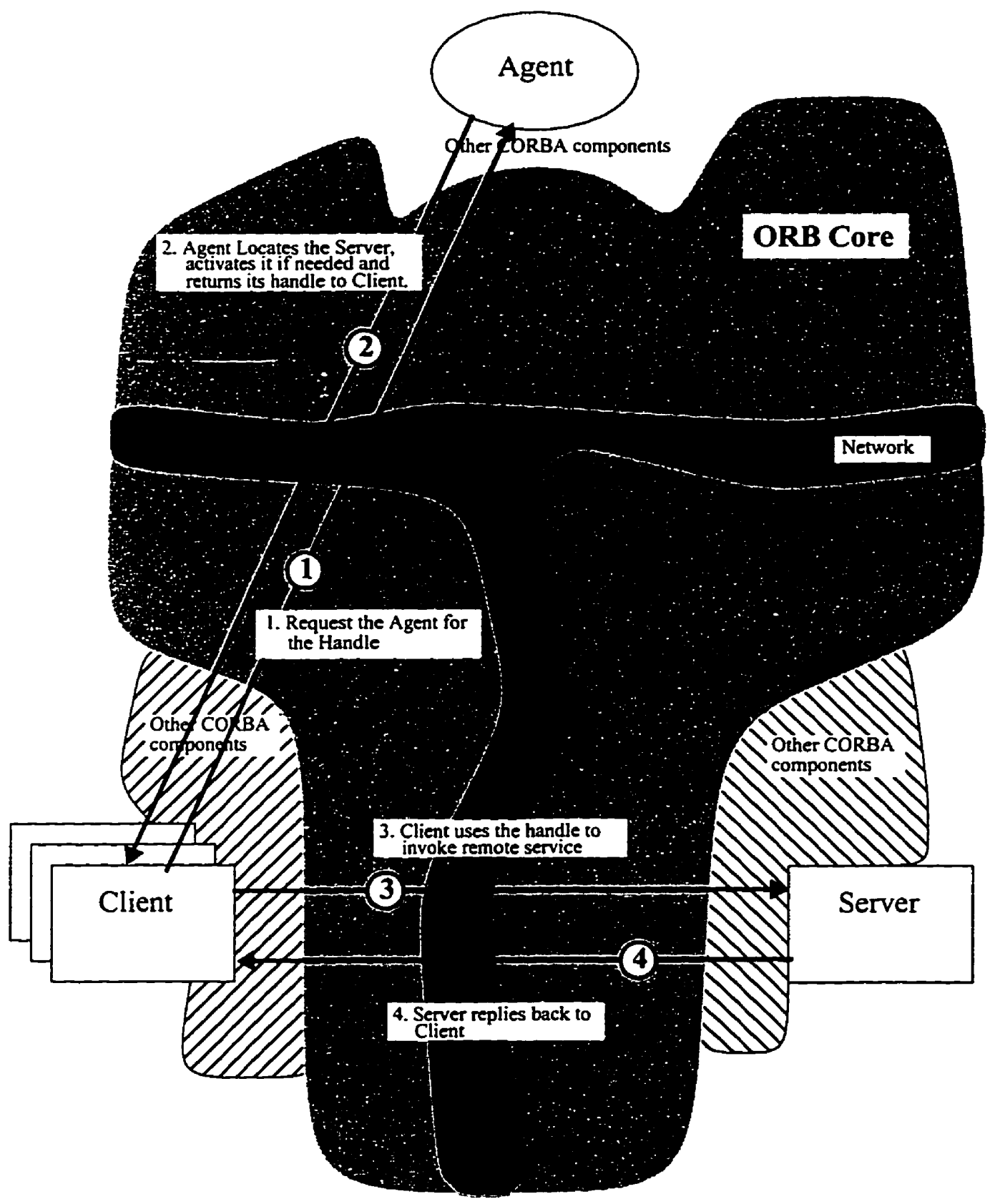

Figure 4-2: $A$ high level interaction description of the H-ORB Architecture. 


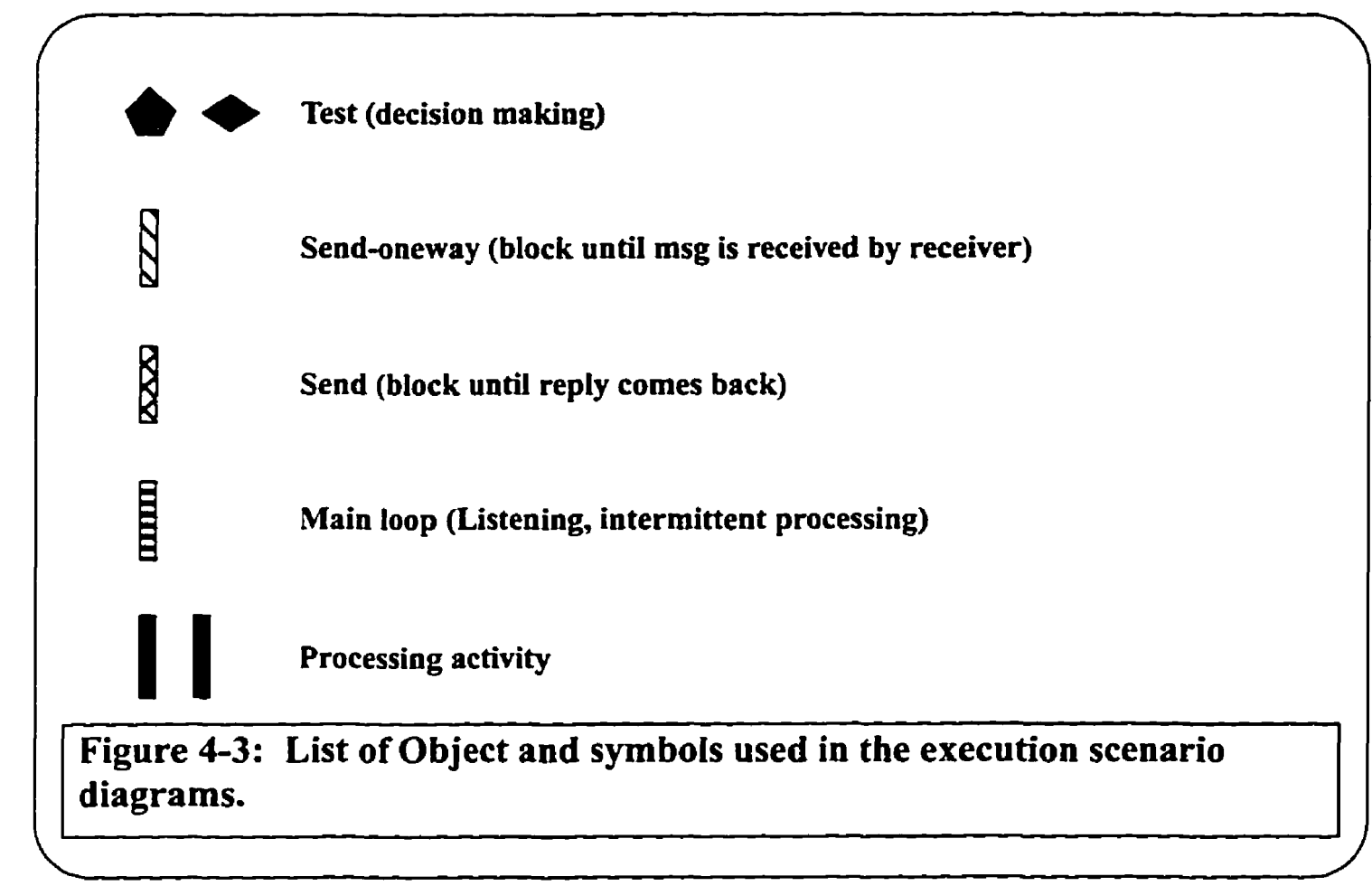

same process), on the same workstation/p.c., connected on the same LAN, or across the galaxy. As described earlier, Object references can not be created or constructed as variables since they are always returned as a result of an operation invocation, hence they can be stringified/destringified for storing persistently or for passing as remote function arguments. Figure 4-2 shows a high level description of this architecture. Since the Agent is a special process, the interface between it and the ORB core is presented by a different shade than that of the client and server processes. Figure 4-3 has a description of the objects used in the execution scenario diagrams of the various ORB architectures discussed in the next subsections.

\subsubsection{The H-ORB Scenario}

The presented scenario describes the general behavior and roles of the components of 
the H-ORB client/server architecture. However, specific implementations may differ slightly. Figure 4-4 illustrates the execution sequence and the interactions among the main components. It should also be mentioned that, in order to focus on the main components only and simplify the diagram, other mediating components are not shown.

\subsubsection{The Client}

1) The client rises and enters a relatively short initialization period, e.g. opening files for input/output, preparing the correct arguments for the service required.

2) The CORBA client must contact the Agent in its domain to obtain the handle, i.e. the object reference, to the sought object implementation before it can invoke methods on it. The Agent, based on the server activation policies described later in Section 4.3.2.3, returns the address of the server if the latter is registered directly with it, otherwise the address of the activation daemon, with which the server has registered itself with, is returned (the process is transparent to the client). The activity of obtaining the handle is called the "bind" operation. As a side-effect of this operation and depending on the bind options, the client has set in its binding request, the ORB core along with other ORB components locate the object implementation process, activate it if currently inactive, then determine when to establish a connection between the client and the object implementation.

3) Upon obtaining the Object Reference the ORB instantiates the C++ class generated in the client side representing the server. Consequently, the stub, will 


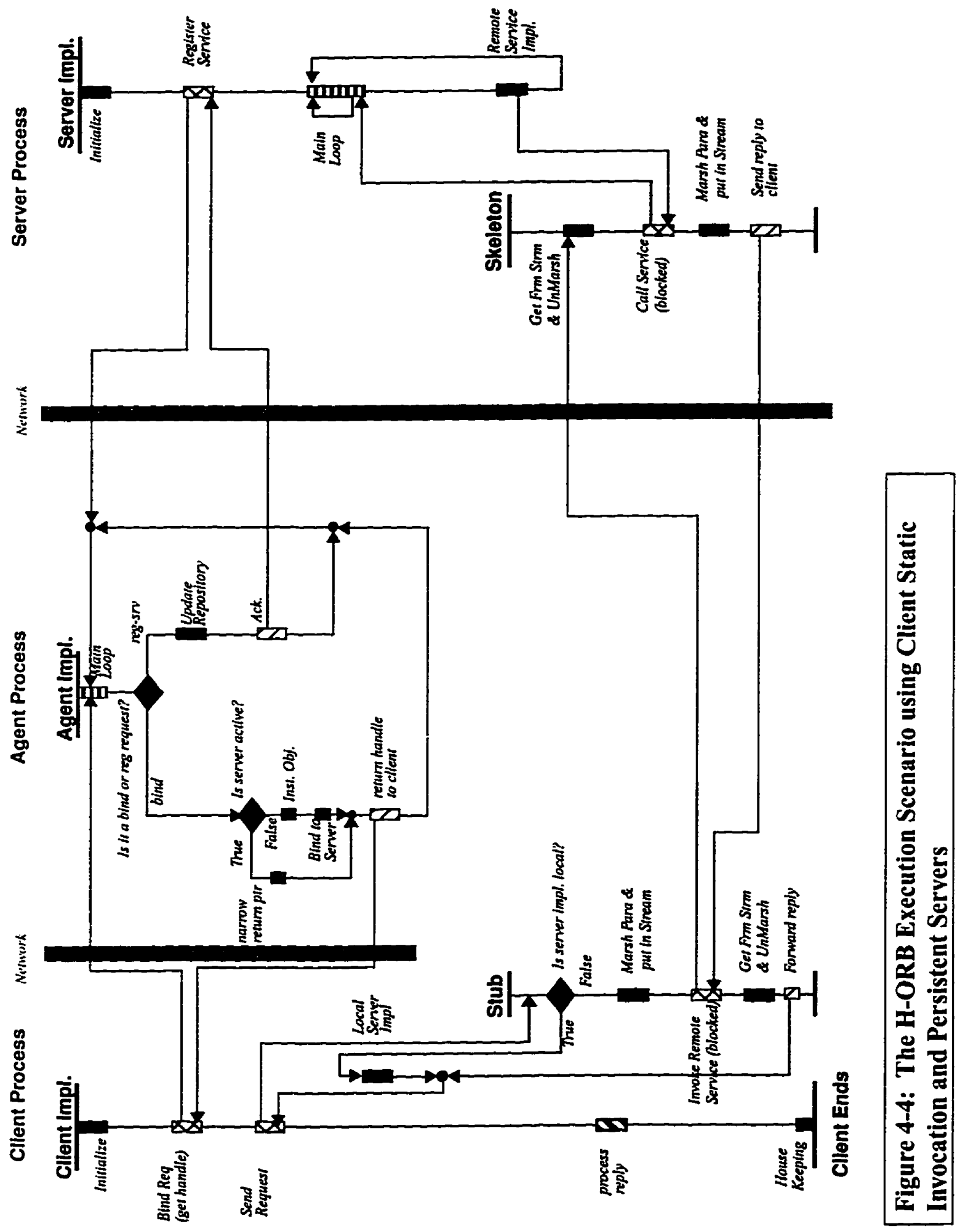


will get invoked when the client uses this handle to invoke the server.

4) When the stub is invoked, it first checks the handle returned by the agent, and in cases where the server is deemed to be "local", residing in the same process as the client, the ORB returns a pointer widened to the client side object interface, and the method invocation is performed as a $\mathrm{C}++$ method invocation (with the ORB core and the network getting bypassed). Whereas if the server is remote, then the stub marshals the passed parameters and puts them in the stream and uses the handle to invoke the remote server, which in turn processes the request and returns the reply if needed.

5) Depending on the semantics of the method invocation defined in the IDL source code, the client may block waiting for the reply from the server, or can proceed to do some other tasks and receive the reply (if any) at its convenience and/or when the reply is available.

\subsubsection{The Agent}

1) After getting activated, the Agent enters its main event loop and waits for incoming messages.

2) When a Registration request comes from a Server, the Agent will use the information in the request to update one or more of its repositories which will be used later for locating and activating the respective servers. Then an acknowledgment message is sent back to the calling server.

3) If a binding request arrives from a client, then the Agent will look for the 
location and the status of the sought server in its repositories. If the server is active, then its reference is returned to the respective client after narrowing it down to have it statically typed to the requested derived interface. Otherwise, if the server is inactive (non-persistent as described in Section 4.3.2.3) then the address of its activation daemon, which performs the actual activation, is retumed (this whole process is transparent to the client).

\subsubsection{The Server (Object Implementation)}

1) If the server is persistent (activated outside $\mathrm{BOA}$ ), then it goes into its initialization phase. Afterwards, the server registers itself with the Agent, through its respective BOA, and waits for the Agent's acknowledgment. Once the acknowledgment is received, the server enters its main event loop: enter a listening mode, and wait for clients requests. The activation must be done in accordance with the CORBA Server Activation Policies, when multiple objects or implementations exist, as listed below.

i) Shared Server: More than one active object of a given implementation share the same server process.

ii) Unshared Server: At any time, there can be no more than one active object of a given implementation in one server process.

iii) Server-per-method: Each method invocation entails the activation of a separate server process which terminates when the method activity is completed. 
iv) Persistent Server: In this case, the server is activated outside its BOA, but still must register itself with the BOA to be able to receive calls from the clients, and it is assumed that it is shared by multiple active objects.

2) If the server is non-persistent (not activated externally), then it must register itself with an activation daemon. This daemon activates the server (if not already active) upon the arrival of a client's request. The server's designated $\mathrm{BOA}$ and daemon cooperate in delivering the client's request to it

3) When a client request arrives at an active server, the respective skeleton receives the request, unmarshals the data, and then invokes the sought method in the object implementation. Based on the semantics of the remote procedure defined in the IDL source code, the skeleton may remain blocked until the service is completed, marshall the reply and send it back to the calling client at which time the server, based on its activation policy, may go back to its main event loop or cease to exist.

\subsection{The Forwarding ORB (F-ORB) Architecture}

This section presents the Forwarding ORB. The name was taken from an earlier paper by Adler [ADL-95]. However, the execution scenario is different. The discussion covers a description of the main components of this architecture as well as its execution scenario.

\subsubsection{Definition}

The $F-O R B$ is an architecture in which the agent forwards the client request to the respective server which in turn performs the needed service. The server replies back 
directly to the originating client. The name of the client, or other identifying data, is embedded within the request so that the reply can be sent back directly without going through the agent. The High level interaction between the main components is shown in Figure 4-5. Since the Agent is a special process, the interface between it and the ORB core is presented by a different shade than that of the client and server processes.

\subsubsection{The F-ORB Scenario}

The scenario below describes the behavior and roles of the components of the F-ORB client/server architecture. Figure 4-6 shows the execution sequence and interaction of the main components of this architecture.

\subsubsection{The Client}

1) The client rises and enters its initialization phase, including the preparation of the needed arguments to be passed to the sought server.

2) The client, in accordance with the CORBA specifications, does not know nor care about the sought server location and must contact the ORB Agent in its domain to reach that server. Unlike the bind operation defined earlier in the $\mathrm{H}$ ORB architecture, the proposed binding operation, called hereinafter " $f$-bind", forwards the client request that includes the service needed and the correct types and values of the passed arguments to the Agent in the local domain of the client. Since the client is not expecting any reply from the Agent, it is logical to implement the f-bind call as a "send-oneway" call which is CORBA's approach for implementing an asynchronous call. As described by 


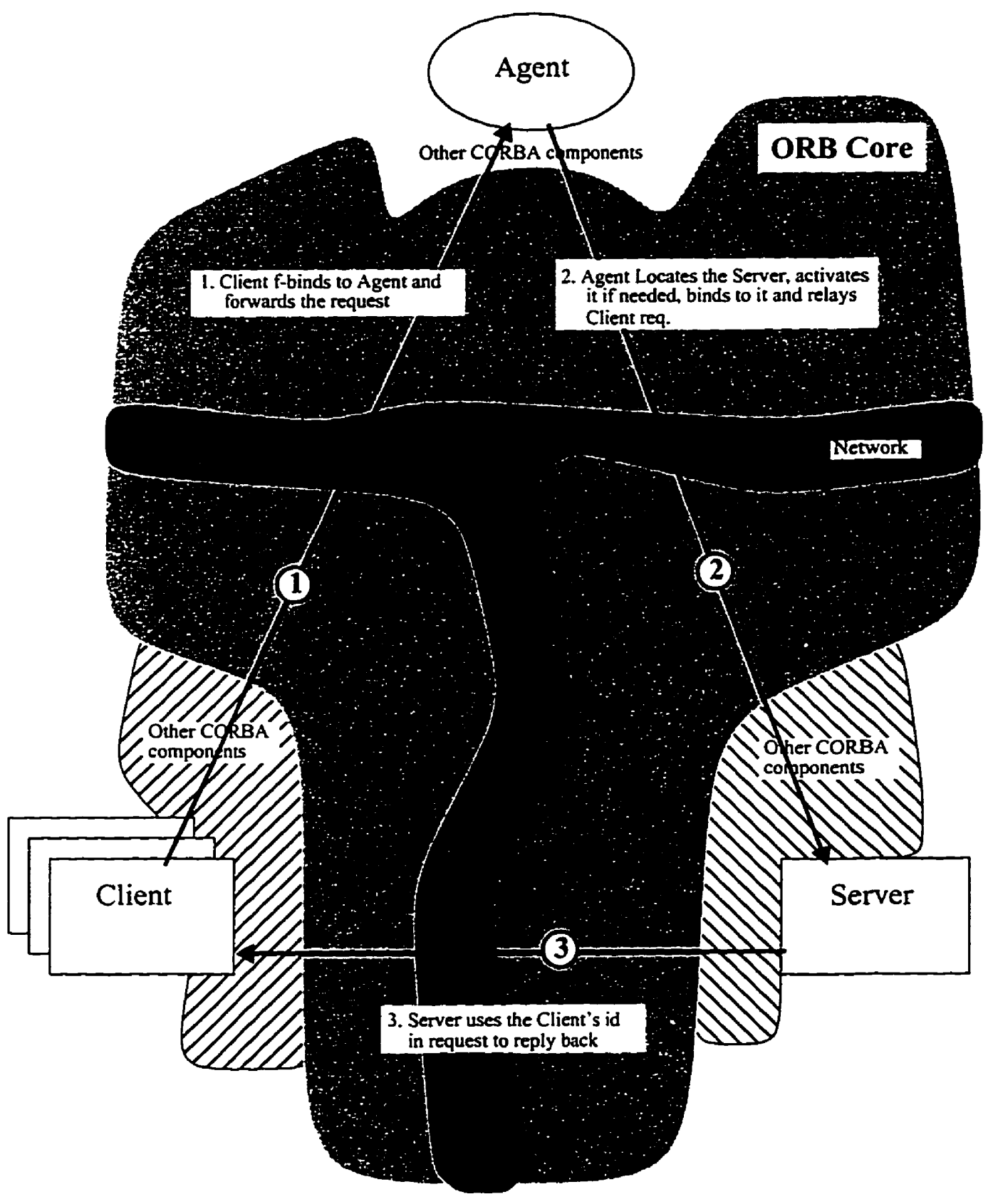

Figure 4-5: A high level interaction description of the F-ORB Architecture. 
Siegel in [SIE-96], the call returns to the client immediately and there is no waiting experienced by the client [SIE-96]. However, we have observed on the ORBeline system that when large amounts of data are transferred the sender can get blocked until the message is delivered to server. Therefore, a sendoneway call is referred to hereinafter as a pseudo-asynchronous call. Upon receiving the request on the Agent's side, the client becomes free to do any further activities.

3) If the client is expecting a reply from the server (or any other process), then it must register itself with the Agent to be accessible. After registration, the client can proceed to do some other tasks, including calling other remote services and receive the reply later (if any) at its convenience and/or when the reply is available, or may choose to wait until the reply arrives from the remote server.

4) Upon the arrival of the reply, the intercepting client skeleton unmarshals the message parameters and passes them to the client.

\subsubsection{The Agent}

1) After getting activated, the Agent enters its main event loop and waits for incoming messages.

2) When a Registration request arrives from a process, the Agent will use the information in the request to update one or more of its repositories which will be used later for locating and activating the respective processes. Then an acknowledgment message is sent back to the calling process. 
3) If an f-binding request arrives from a client, then the Agent will extract the information needed to identify for the server from the message. The Agent looks for the location and the status of the sought server in its repositories. If the server is active, then its reference is narrowed down to have it statically typed to the requested derived interface. Otherwise, if the server is inactive (non-persistent as described in Section 4.3.2.3) then the address of its activation daemon, which performs the actual activation, is used to activate it. The Agent marshals the request parameters and uses the server handle obtained to send the message to the originally sought server using the send-oneway mode described in Section 4.4.2.1. As soon as the server skeleton gets the message, the Agent is freed and will enter its main event loop.

4) If a binding request arrives from a client, then the Agent will look for the location and the status of the sought server in its repositories. Depending on the status of the server, being active or inactive), the needed handle is obtained following the same approach as in step (3) above and returned to the calling client. The Agent is freed and ready to enter its main event loop.

\subsubsection{The Server (Object Implementation)}

1) If the server is persistent (activated outside BOA), then it goes into its initialization phase. Afterwards, the server registers itself with the Agent, through its respective BOA, and waits for the Agent's acknowledgment. Once the acknowledgment is received, the server will enter its main event loop: enter 


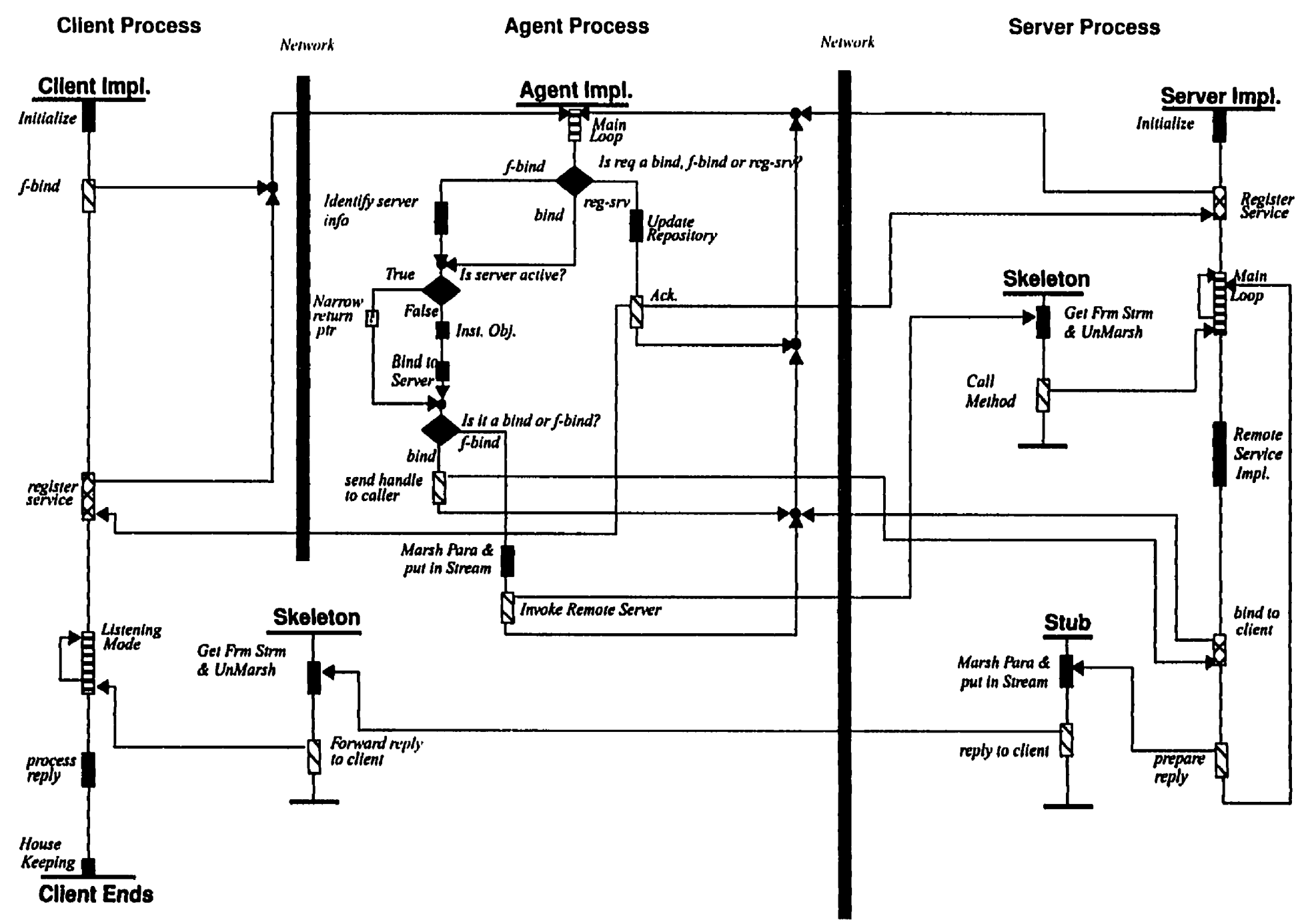

Figure 4-6: The F-ORB Execution Scenario using Client Static
Invocation and Persistent Servers 
a listening mode, and wait for clients requests.

2) If the server is non-persistent (not activated externally), then it must register itself with an activation daemon. This daemon activates the server (if not already active) upon the arrival of a client's request. This activation must be done in accordance with the CORBA Server Activation Policies stated in the $\mathrm{H}-\mathrm{ORB}$ architecture in Section 4.3.2.3, when multiple objects or implementations exist. The server's designated BOA and daemon cooperate in delivering the client's request to it.

3) When a client request, relayed from the Agent, arrives to an active server, the skeleton will intercept the request, unmarshall the data and then call the sought method in the object implementation.

4) Upon completing the request processing, a reply is sent if the client is expecting one using the send-oneway mode described earlier. Based on the client name (or other identifying data) embedded in the request, the server must obtain the client's handle and use it to send the reply. Getting the handle can be achieved in one of the following approaches:

i) The handle can be obtained, stringified and embedded in the request by the Agent prior to relaying the request to the server. Hence the server would need only to unstringify the handle and use it.

ii) A smart server would obtain the respective handle of the originating client from the Agent and store it locally for future invocations. 
iii) A simple server would call the Agent each time a request arrives to get the handle.

5) The server uses the handle to send the reply back to the client through the server stub after marshalling the parameters.

6) The server based on its activation policy, may go back into its main event loop or terminate.

\subsection{The Process Planner ORB (P-ORB) Architecture}

This section presents the Process Planner ORB. The name was taken from an earlier publication by Adler [ADL-95] and applied to CORBA. The presentation includes a description of the main components of this architecture in addition to its execution scenario.

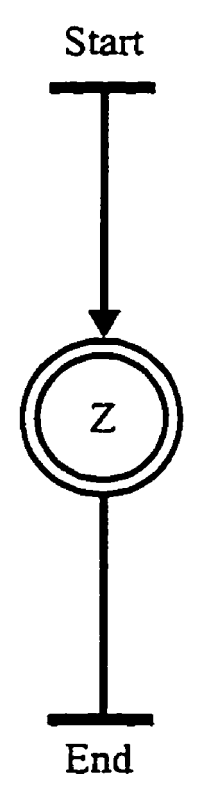

A Process is executing a composite request " $\mathrm{Z}$ "

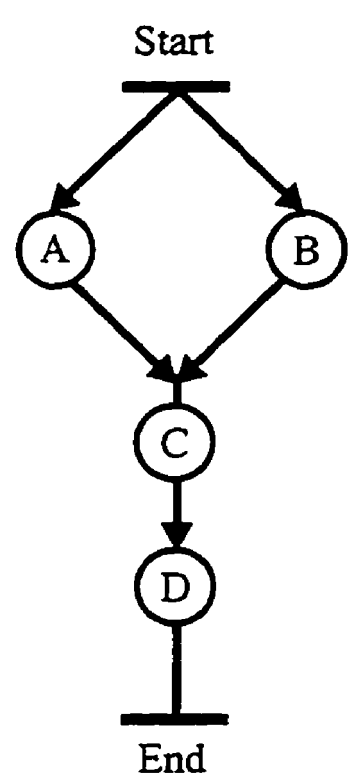

The actual processing of request "Z" through its constituent components

Figure 4-7: A composite request and its constituent components. 


\subsubsection{Definitions}

\section{The Composite Request}

A composite request consists of multiple single requests with precedence relationships among them. The composite request " $Z$ " shown in Figure 4-7 is actually processed by its constituent services. In this example the services $A$ and $B$ are independent of each other and can be executed concurrently. Service C on the other hand is a successor of A \& B and a predecessor of service D. In practice, composite requests can be far more complex than this example.

\section{The P-ORB}

The $P$-ORB is an architecture in which the agent decomposes the client composite request into the respective constituent services, establishes a record of dependency among them, coordinates the invocation of the respective servers utilizing the maximum degree of concurrency based on the dependency constraints and the underlying software (ability of forking processes or spawning threads) and hardware (single/multiple processor) platforms. When all the constituent services are done, the agent prepares a single reply and sends it back to the originating client.

One approach of decomposing a composite request is integrating a script interpreter with the agent [ADL-95]. Upon receiving the client's request, the agent instantiates the respective script and executes the individual script steps in sequence (updating interim variables and completing dependency constraints tests as necessary). Script steps can be atomic steps (requests for constituent services) or compound steps that combine atomic 
steps with conditional branching and iterative loops. Steps can also be invocations of other nested/embedded scripts. Upon completing the script, the agent returns the results to the client. If the same request is to be served by an H-ORB or an F-ORB, then multiple single request/reply cycles are needed to execute those constituent services sequentially. A highlevel overview is shown in Figure 4-8.

\subsubsection{The P-ORB Scenario}

The scenario below describes the proposed behavior and roles of the components of the P-ORB client/server architecture. Figure 4-9 shows the execution sequence and the interactions among the main components.

\subsubsection{The Client}

1) The client rises and enters its initialization phase, including the preparation of the needed arguments to be passed to the sought server.

2) The client, in accordance with the CORBA specifications, does not know nor care about the sought server location and must contact the ORB Agent in its domain to reach that server. Unlike the bind operation defined earlier in the HORB architecture, the proposed binding operation, called hereinafter "p-bind", forwards the client composite request that includes the service needed along with the proper types and values of the passed arguments to the Agent in the local domain of the client. Since the Agent is designed to handle multiple requests/replies from clients and servers, it is logical to implement the p-bind call as a "send-oneway" call. Upon receiving the request on the Agent's side, 


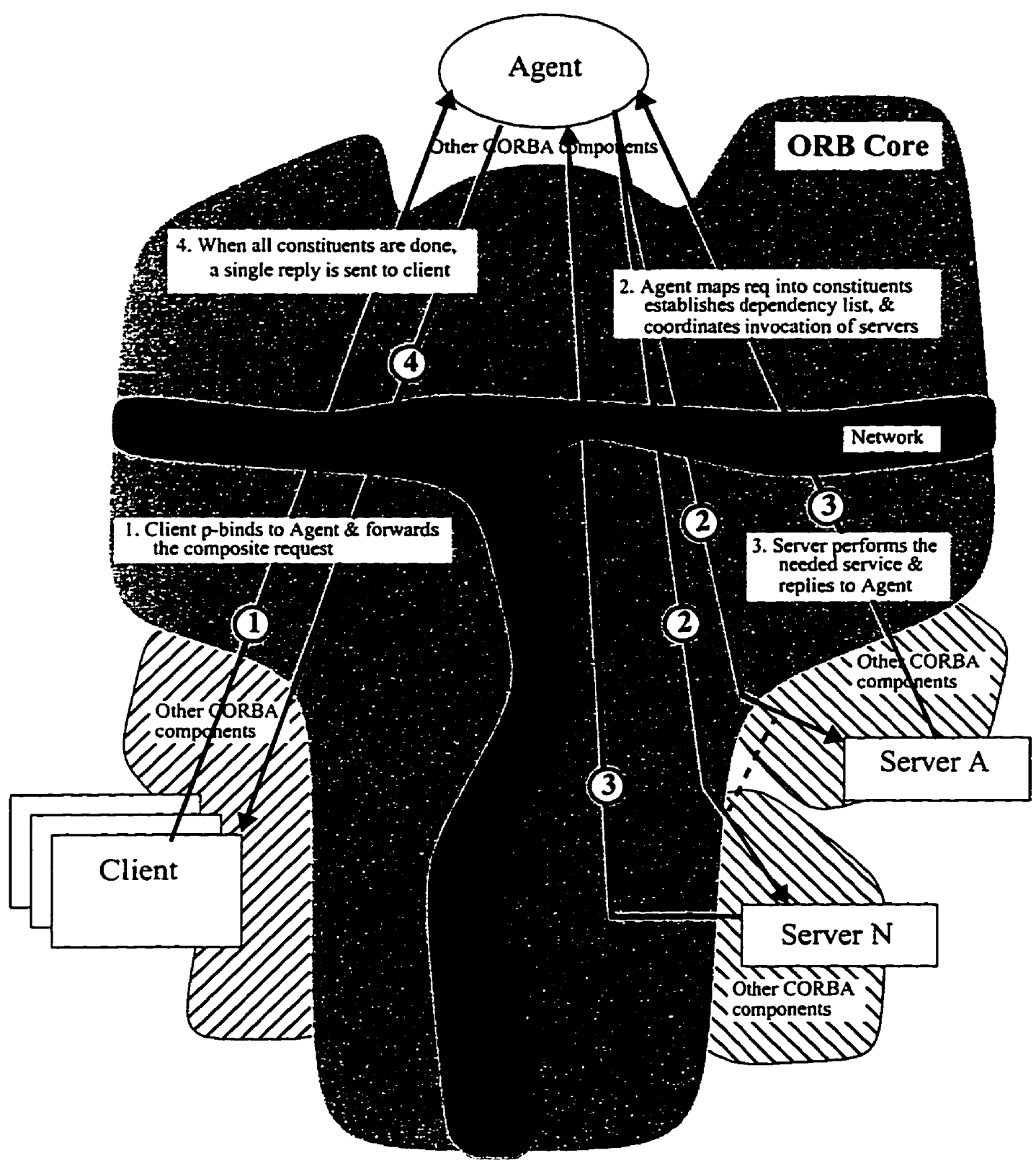

Figure 4-8: $A$ high level interaction description of the P-ORB Architecture. 
the client becomes free to do any further activities.

3) If the client is expecting a reply from the Agent (or any other process), then it must register itself with it to be accessible. After registration, the client can proceed to do some other tasks, including calling other remote services and receive the reply (to the composite request) later (if any) at its convenience and/or when the reply is available, or may choose to wait until the reply arrives from the Agent.

4) Upon the arrival of the reply, the intercepting client skeleton unmarshals the message parameters and passes them to the client.

\subsubsection{The Agent}

1) After getting activated, the Agent enters its main event loop and waits for incoming messages.

2) When a Registration request comes from a process, the Agent will use the information in the request to update one or more of its Repositories which will be used later for locating and activating the respective processes. Then an acknowledgment message is sent back to the calling process.

3) If a p-binding request arrives from a client, then the agent decomposes the composite request into its constituent services. The Agent identifies the servers capable of providing these constituent services, and establishes a record of dependency constraints among them.

4) Afterwards, the Agent gets the handle to all servers whose constituent services 
comply with the current dependency constraints, and dispatches all respective requests using the send-oneway mode, exploiting the maximum level of permitted concurrency, and embedding the Agent's name (or other identifying data) in the request.

5) The Agent enters the listening mode (main event loop) and waits for new client requests or replies from the sought servers. Whenever a reply comes back from a server, the dependency list of the respective request is updated, and step (4) above is repeated until no further constituent services remain.

6) The Agent assembles the final reply in a single form and sends it back to the originating client using the send-oneway mode.

7) The Agent enters its main event loop.

\subsubsection{The Server (Object Implementation)}

1) If the server is persistent (activated outside BOA), then it goes into its initialization phase. Afterwards, the server registers itself with the Agent, through its respective BOA, and waits for the Agent's acknowledgment. Once the acknowledgment is received, the server will enter its main event loop: enter a listening mode, and wait for clients requests.

2) If the server is non-persistent (not activated extemally), then it must register itself with an activation daemon. This daemon activates the server (if not already active) upon the arrival of a client's request. This activation must be done in accordance with the CORBA Server Activation Policies stated in the 


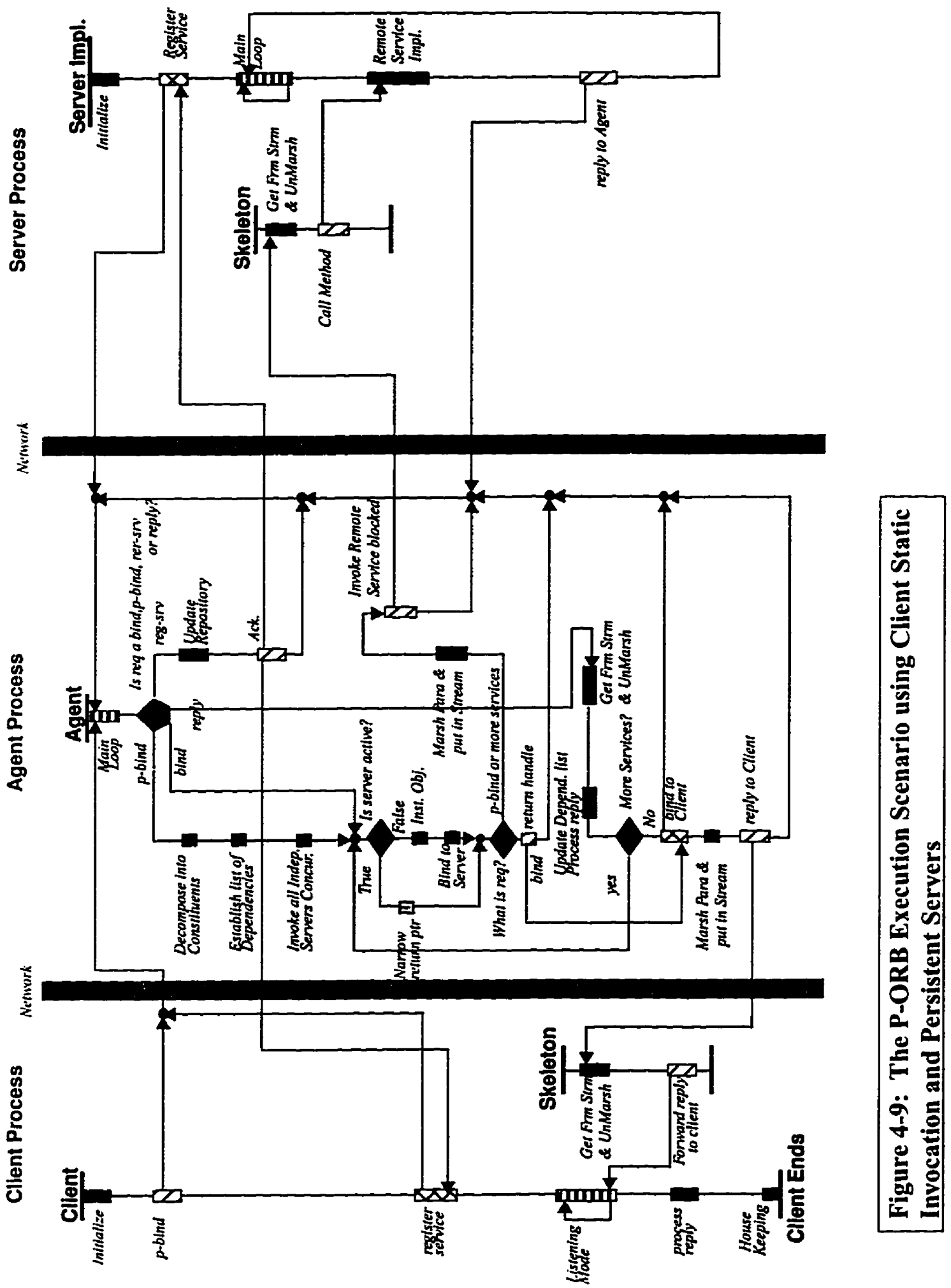


H-ORB architecture in Section 4.3.2.3, when multiple objects or implementations exist. The server's designated BOA and daemon cooperate in delivering the client's request to it.

3) When a constituent request, sent from the Agent, arrives at an active server, the skeleton receives the request and unmarshals the data then calls the sought method in the object implementation.

4) Upon completing the service and if the client is expecting a reply, then based on the Agent's identifying data embedded in the request, the server must reply back to the calling Agent using the Agent's id embedded in the request.

5) The server based on its implementation policy may go back into its main event loop or exit. 


\section{Chapter 5 Comparison of System}

\section{Performance}

\subsection{Case Study}

The application system is a banking system which offers two types of accounts, $a$ Checking account and a Savings account. The bank maintains two independent classes of servers. For the purpose of load balancing, Class $A$ is comprised of two identical checking servers, $A \boldsymbol{I}$ and $A 2$. Class $B$ is also comprised of two identical saving servers $B 1$, and $B 2$. A client request asks for the current balance of both of his accounts. For the purpose of investigating the performance analysis of the system, no implementation of the corresponding data bases was done. Instead, nested for loops are used to represent the request processing at the server. The next two subsections cover the workload and the experimental environment. The request processing steps will vary depending on the underlying CORBA architecture and will be described in details as well. The three main architectures are the H-ORB, the F-ORB and the P-ORB.

\subsubsection{The Workload}

The construction of the workload is based on specifying the characteristics of the system as well as those of the applications executing on it that have a significant effect on the system performance.

The main attributes that were investigated are as follow: 
1) Message Size $(L)$ : This is the length of the message (total number of bytes) the client sends per request to the sought server which in turn sends it back and is represented by $\mathrm{L}$. In all case studies, the message size was kept constant while propagating among all participating processes in each experiment. As shown in the subsequent sections $L$ has a significant effect on the performance of the three architectures.

2) Request Service Times $S_{A}$ and $S_{B}$ : This is the total CPU demand that each client request would need at each class of servers. It represents the actual processing time only and does not include any other overheads such as binding or marshalling etc. Whether or not loading the servers of both classes has an effect on the relative performance of the three architectures is an important question.

3) Number of clients $(N)$ : This is the total number of active clients during the life of the experiment. As illustrated in Section 5.2, increasing the number of clients reveals the relative scalability of the three architectures, another issue that deserves studying.

4) Inter-Node Communication Delay (D): Client nodes may be several nodes away from agent and server nodes in a geographically dispersed distributed system. Since store and forward mechanism is used at intermediate nodes in the path between the client, agent, and servers the delay is expected to increase with the number of such intermediate nodes. How much impact the delay in message delivery has on the relative performance of the three architectures 
warrants investigation. The delay experienced by a particular request is simulated by forcing the sending process to sleep for $D$ time units since the available LAN in the RADS lab is a 10 Mbps Ethernet LAN.

Table 1: Levels for the Workload Factors

\begin{tabular}{|l|l|}
\hline \multicolumn{1}{|c|}{ Factors } & \multicolumn{1}{c|}{ Levels } \\
\hline$N$ & $1, \ldots \ldots, 24$ \\
\hline$D$ (msec) & $200,250,500,1000$ \\
\hline$L$ (bytes) & $4800,9600,19200$ \\
\hline$S_{A} / S_{B}$ (msec) & $10 / 15,50 / 75,250 / 375$ \\
\hline
\end{tabular}

By using different combinations of $\mathrm{S}_{\mathrm{A}}, \mathrm{S}_{\mathrm{B}}, \mathrm{D}$, and $\mathrm{L}$ it is possible to investigate both computation bound and communication bound systems. In a computation bound system, the server are heavily loaded whereas the message sizes are small. A communication bound system is characterized by large messages and/or long inter-node delays but small service times for client requests. The different levels of the factors used in the generation of workload in the experiments are listed in Table 1. The number and choice of the levels for each factor reflect a compromise between representativeness and an acceptable run time for the experiments.

Degree of Cloning: Another important parameter is the degree of cloning used for the agent process that was varied from 1 (no cloning), to 4, to 8 . A clone of a process is its copy that shares a message queue with its parent. Thus, with a set of process clones one process can process an outstanding message when the others are busy. Since the H-ORB uses the ORBeline agent, we did not have any control over the agent process. Therefore, it will 
maintain its no-cloning status and the issue of cloning is applied only to the F-ORB and PORB. By cloning the agent process, the waiting time for a server is reduced: even though one agent process is tied up in an interaction another free agent process can interact with a responding server.

Client Request Path: This is the path along which the client request will go through from the instant of initiating the request on the client node, passing through all intermediate nodes, getting processed in the sought server on the destination node and all the way back to the originating client. As described earlier, each client is assumed to require to one service from both of the server classes A and B and these services are assumed to be independent of each other and can be invoked concurrently. The client request path and nature will vary depending on the underlying ORB architecture in addition to the type of the remote service invocations. In all the architectures, the Static invocation (more details in Chapter 3 Subsection 3.3.3) approach is used. The respective client request path is presented in the coming subsections.

\subsubsection{Experimental Environment}

The System in all the architectures is comprised of a total of (14) Sun workstations ranging from Sparc-2 to SparcUltra connected over a $10 \mathrm{Mbps}$ Ethernet LAN. A high level description of three different architectures for client/server interaction is provided in Chapter 4. A lower level description of their behaviors is presented in the following subsections. 


\subsubsection{The Handle-Driven ORB H-ORB System}

The System in this architecture consists of a number of Nodes as the main components. These nodes are distributed and interconnected via a 10Mbps Ethernet, and are described along with the respective request path.

a The Client Nodes: Each node is a Sun workstation that is chosen from a pool of available workstations including Sparc5,10, 20 and Ultras. A fixed number of identical client processes are distributed over the available nodes and run in a batch (closed) environment. Each client is designed to run for a predefined number of requests (cycles). Whenever a client finishes its cycle, it starts immediately another identical cycle, i.e. there is no think time. Due to the nature of the default agent's behavior (returning the handle of the sought server), a typical client cycle consists of accessing the checking account (class A) first then the saving account (class B). Since the clients have very little computational demand on their own nodes (workstations), negligible differences have been observed in the cycle time of the clients that are distributed over a range of heterogeneous nodes.

b- The Server Nodes: This architecture has a total of four server nodes. Each node is a Sparc-2 workstation. As described earlier, there are two classes of CORBA objects (servers), class A and class B. Each class has two instances, hence there is one server process per machine.

c- The Agent Node: This is a Sparc-20 workstation where the default agent is located and no clients/servers reside. It is connected to the clients and servers via the same ethernet described earlier. The agent is the dynamic directory server described in 
Chapter 4 in Section 4.1. According to the ORBeline specifications, there can be only one active agent in each user domain and this is the default agent supplied by ORBeline. It should be emphasized that the default agent distributes the requests among all the servers that belong to a certain class equally (by returning the handle of the server chosen using the round-robin approach) unless the client has identified a specific instance of the server (e.g. based on its name, host node or the ORB vendor) at which time the default agent returns the handle of that particular instance.

d - The H-ORB Request Path: As described earlier, for a client to reach a CORBA object (server), it must obtain its handle. The complete client Request Sequence Path per cycle is shown in Figure 5-1 and the path components (that are numbered in the figure) are explained below. It should be noted that all client calls are symchronous, hence the client is blocked waiting for the reply (be it from the default agent or the server).

- Steps 1-2: Obtaining the handle for a server of class $A$ from the default agent. Let $A I$ be the server, the handle of which is returned. The client sleeps twice (first before sending to the default agent and second after receiving the handle) to simulate the preset network inter-node delay.

- Steps 3-4: Invoking server $A 1$ and obtaining the reply. The client sleeps before sending the request to $A l$, while $A l$ sleeps prior to sending the reply to simulate the preset network inter-node delays.

- Steps 5-6: Obtaining the handle for a server of class $B$ from the default agent. Let $B I$ be the server, the handle of which is returned. The client sleeping habits 


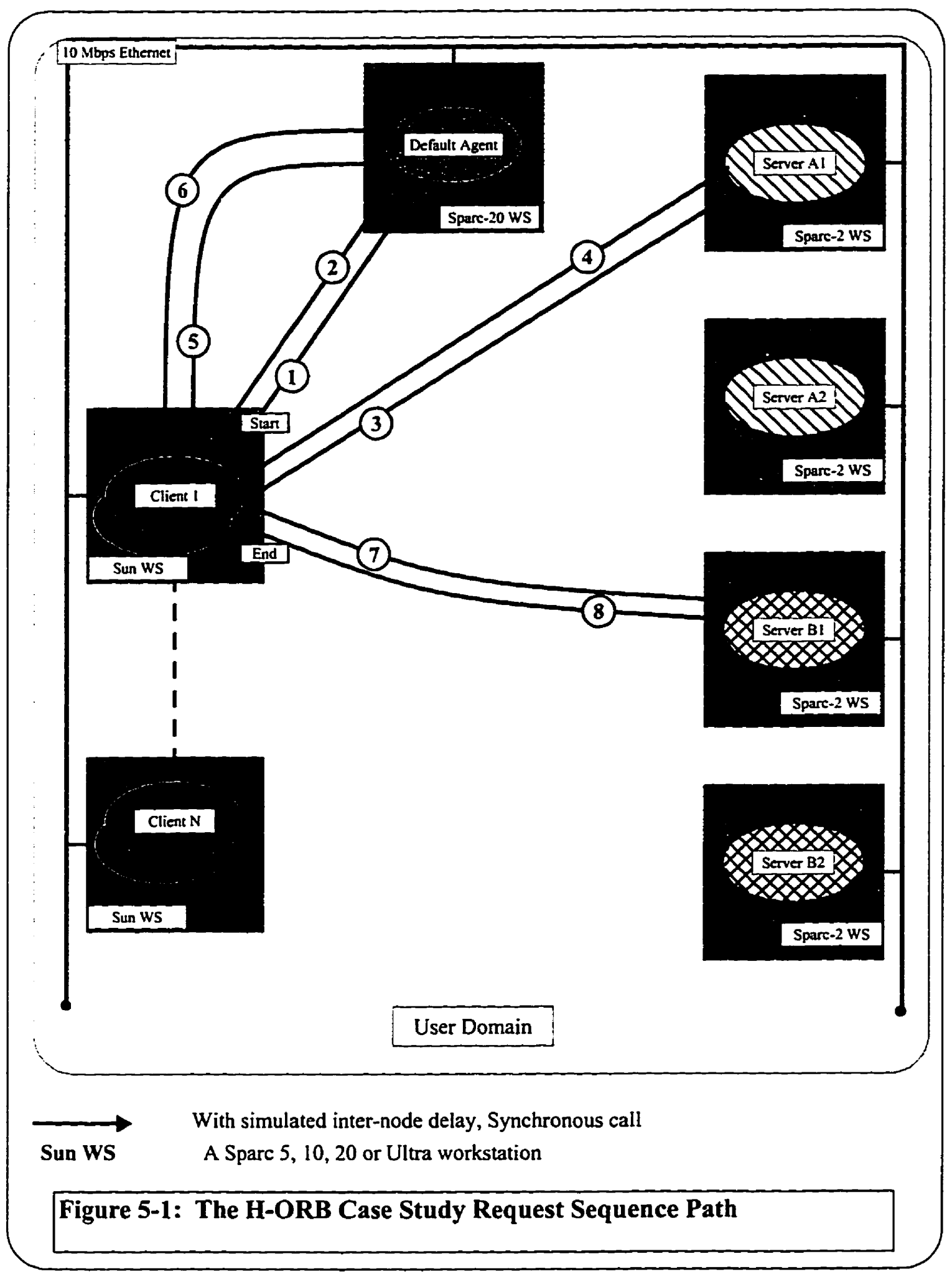


are identical to the ones in steps $1-2$.

- Steps 7-8: Invoking server $B I$ and obtaining the final reply which indicates the end of the client request. The sleeping behavior of both the client and $B I$ are identical to those mentioned in steps $3-4$ above.

\subsubsection{The Forwarding ORB F-ORB System}

The F-ORB architecture differs from the previous architecture in the sense that the $f$ agent forwards the reply to the sought server rather than returning the handle to the requesting client (more details are given in Section 4.4 in Chapter 4). Two versions of the F-ORB architecture, F-ORB Version 1 (F-ORB.VI) and Version 2 (F-ORB.V2), are implemented. The interaction among clients, agents, servers are different in each version. A brief discussion of the differences between the two architectures is presented.

1) The Client/Agent Interaction: Ideally, an F-ORB client should not ask the default agent for a handle to the f-agent since both agents are integrated into a single process. Instead, it should be able to reach the f-agent by simply invoking the f-bind operation (defined in Chapter 4, Subsection 4.4.2.1). Since access to ORBeline source code was not available the f-agent is achieved by incorporating extra functionalities that interact with the default agent. An FORB.VI client achieves this goal by obtaining the handles to all active fagents during an initialization period (details are in the respective request path description in this subsection), storing them locally, then selecting one randomly (for load balancing) and using that handle to send the request. An F- 
ORB.V2 client contacts the default agent in each request to obtain the handle for the f-agent. No simulated inter-node delays are involved in this call (to make it resemble an f-bind operation) and the load balancing is guaranteed by the default agent (since it returns the handle of an f-agent selected by a round robin approach). The F-ORB.V1 interaction behavior is may be preferable for cases where the overhead of the frequent calls for binding is expensive. FORB.V2 in which the default agent is contacted for every request provides a better way of fault tolerance and load balancing.

2) The Server/Agent Interaction: Due to the send oneway call nature (used in the F-ORB architectures), an F-ORB server needs the handle of the originating client to be able to return the reply. Ideally, the handle should be obtained and stringified by the f-agent and embedded in the request message sent to the server. The server in the F-ORB.VI architecture does that by performing a client-based initialization. When a client first request arrives, the server obtains its handle from the default agent and stores it locally. For subsequent requests from the same client, the server searches for the handle locally and use it. On the other hand, a server in an F-ORB.V2 must ask the default agent for the handle each time a request arrives (no inter-node delays are included to make the behavior closer to the ideal case). The F-ORB.VI behavior is suitable if the number of clients is small. However, as the number of clients becomes larger the F-ORB.V2 is more realistic especially if the agent node is not far away.

Both architectures were run and an in-depth analysis of their performance is presented 
in the Experimental Results section in this chapter. A detailed description of the related nodes and the request path steps is provided here,

a The Client Nodes: The clients are distributed over a pool of available workstations as in the previous architecture. Details are in Subsection 5.1.2.1.

b - The Server Nodes: The server nodes are identical to those described in Subsection 5.1 .2 .1

c- The Agent Node: This is the Sparc-20 workstation described in the H-ORB architecture. This node will have the default agent as well as the $f$-agents. During each experiment, a fixed number of f-agents are activated and set ready to receive and process any client request in cooperation with the default agent supplied by ORBeline. Two versions of the F-ORB architecture are implemented. For both architectures, as explained earlier in the workload in Subsection 5.1.1, the clients, the f-agents, and the servers need to obtain the handle of the sought process to be able to forward the request to it. Having no access to the source code of the default agent has made impossible to modify it to include the functionalities of the f-agent in it. Therefore, the co-existence of both the default-agent and the f-agents on the same workstation is necessary to simulate their integration into a single complex agent. Hence, the default-agent presence is mandatory in this architecture.

d - The F-ORB.VI Request Path: The request in this architecture follows a different path in comparison with the $H-O R B$ architecture. The f-agent, is the process which relays the request to the sought server of either class. The initialization phases and the 


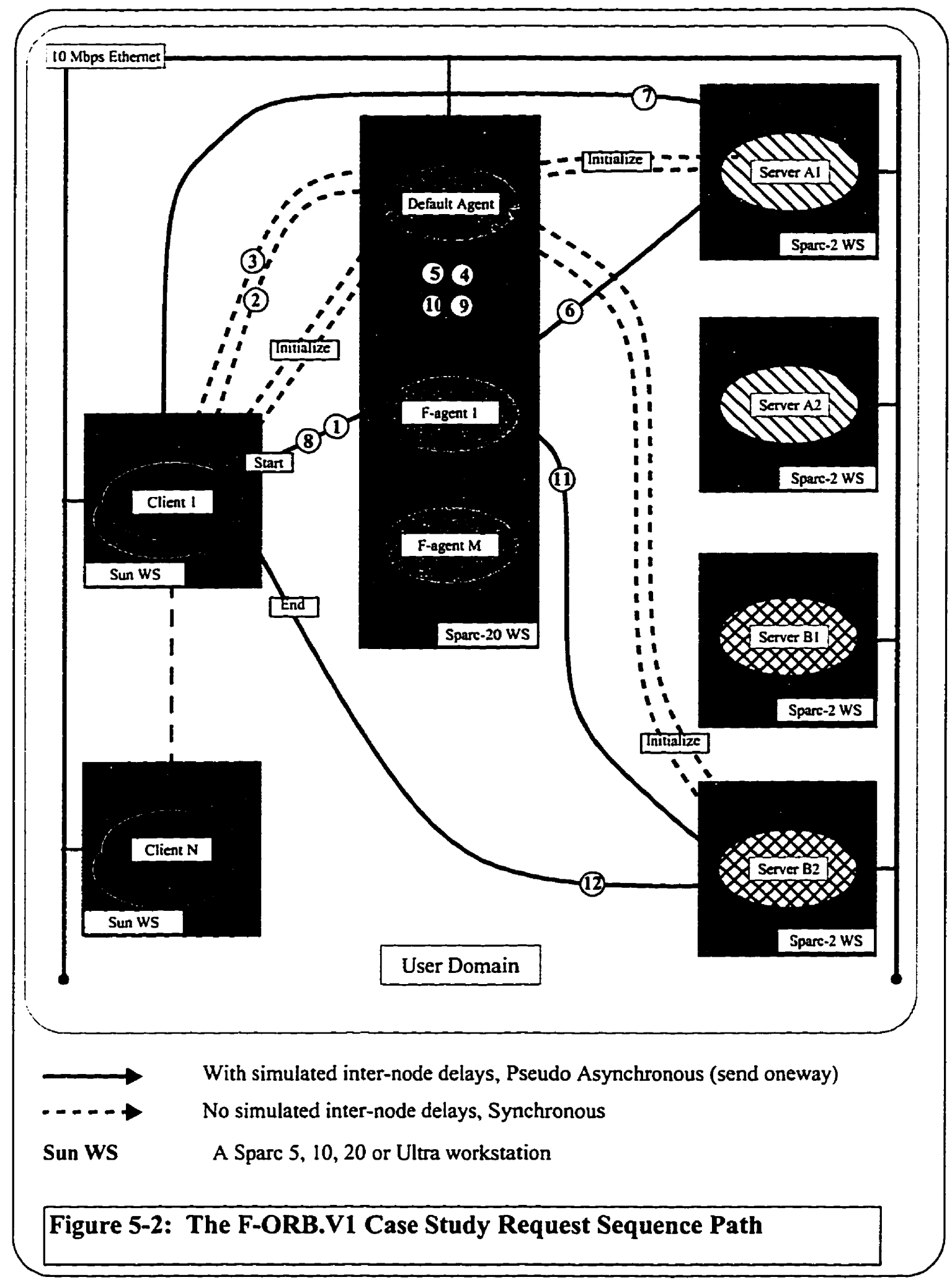


path steps that are numbered in Figure 5-2 are described below. It should be noted that all of the path steps, excluding the initialization phase, use ORBeline's Pseudo Asynchronous (send oneway) calls to the called process. No simulated delays are included in any initialization operation.

- Client Initialization: In practice, the client should not ask the default agent for a handle to an f-agent since both agents (the default and the f-agent) are integrated into a single process (complex agent) and can be contacted via a special ORB function call (the f-bind operation). Hence to make this architecture closer to the ideal F-ORB, the client obtains the handles for all the available f-agents and stores them locally.

- Server Initialization: Ideally, when a client request arrives at a server, its handle has already been stringified and embedded in the message by the agent. The server only needs to unstringify the handle and use it to return the reply. However, this approach, when implemented, was unsuccessful for medium to high system loads due to the problems raised up by ORBeline and Solaris. To simulate this ideal behavior, the server obtains the client's handle from the default agent while servicing the client's first request and stores it locally. For subsequent requests from the same client, the server searches its local data base to find out the respective client's handle and uses it to return the reply to the originating client.

- Step 1: The client selects an f-agent, randomly, and uses its handle to send the request (to resemble the $\mathrm{f}$-bind operation described in Chapter 4 , Subsection 
4.4.2.1). Let $\boldsymbol{F}$-agent 1 be the agent selected randomly by the client.The client forwards the request to the $F$-agentI using ORBeline's send oneway pseudo asynchronous call. The client sleeps before sending to the f-agent to simulate the preset inter-node delay.

- Steps 2-3: The client registers itself with the default agent in order to be accessible to the server processes in the domain. No simulated inter-node delays are involved. This is done only once during the lifetime of the experiment.

- Steps 4-5: F-agentI obtains handle for a server of class $A$. Let $A I$ be the server, the handle of which is returned. This dynamic binding is needed to achieve load balancing and fault tolerance as well.

- Step 6: F-agentI uses the handle obtained in (4-5) to relay the request to $A I$ in a pseudo asynchronous mode. The f-agent sleeps before sending to $A I$ to simulate the preset network inter-node delay.

- Step 7: In order for server $A I$ to return the reply (after processing the request) to the originating client, it must first have its handle. As discussed in the server initialization, the server $A I$ uses the respective handle, stored locally during the client's first request, to return the reply to the originating client using the same pseudo asynchronous mode. $A I$ sleeps prior to sending the reply to simulate the preset network inter-node delay.

- Step 8: The client uses $F$-agentl's handle to forward its request (destined for a class $B$ server) using a pseudo asynchronous call to $F$-agent $l$. The client 
sleeping behavior is identical to that of stepl.

- Steps 9-10: F-agentl obtains the handle for a class $B$ server. Let $B 2$ be the server, the handle of which is returned. This dynamic binding is needed to achieve load balancing and fault tolerance as well.

- Steps 11: F-agent1 uses the handle obtained above to relay the request to $B 2$ in a pseudo asynchronous mode. The agent sleeping behavior is similar to that in step 6.

- Step 12: In order for server $B 2$ to return the reply (after processing the request) to the originating client, it must first have its handle. As discussed in the server initialization, $B 2$ uses the respective handle, stored locally during the client's first request, to return the reply to the originating client using the same pseudo asynchronous mode. $B 2$ sleeps prior to sending the reply to simulate the preset network inter-node delay. The request cycle is complete when the reply message is received by the client

e - The F-ORB.V2 Request Path: The request in this architecture follows a different path in comparison with the F-ORB.VI architecture. The f-agent is the same process which relays the request to the sought server of either class. The path steps that are numbered in Figure 5-3 are described below. It should be noted that some of the steps include ORBeline's Pseudo Asynchronous (send oneway) calls to the called nodes:

- Steps 1-2: This architecture adopts a different approach to simulate the ideal fbind operation (discussed in Chapter 4 in Subsection 4.4.2.1) by obtaining the handle for an $f$-agent from the default agent (in each request cycle) without 


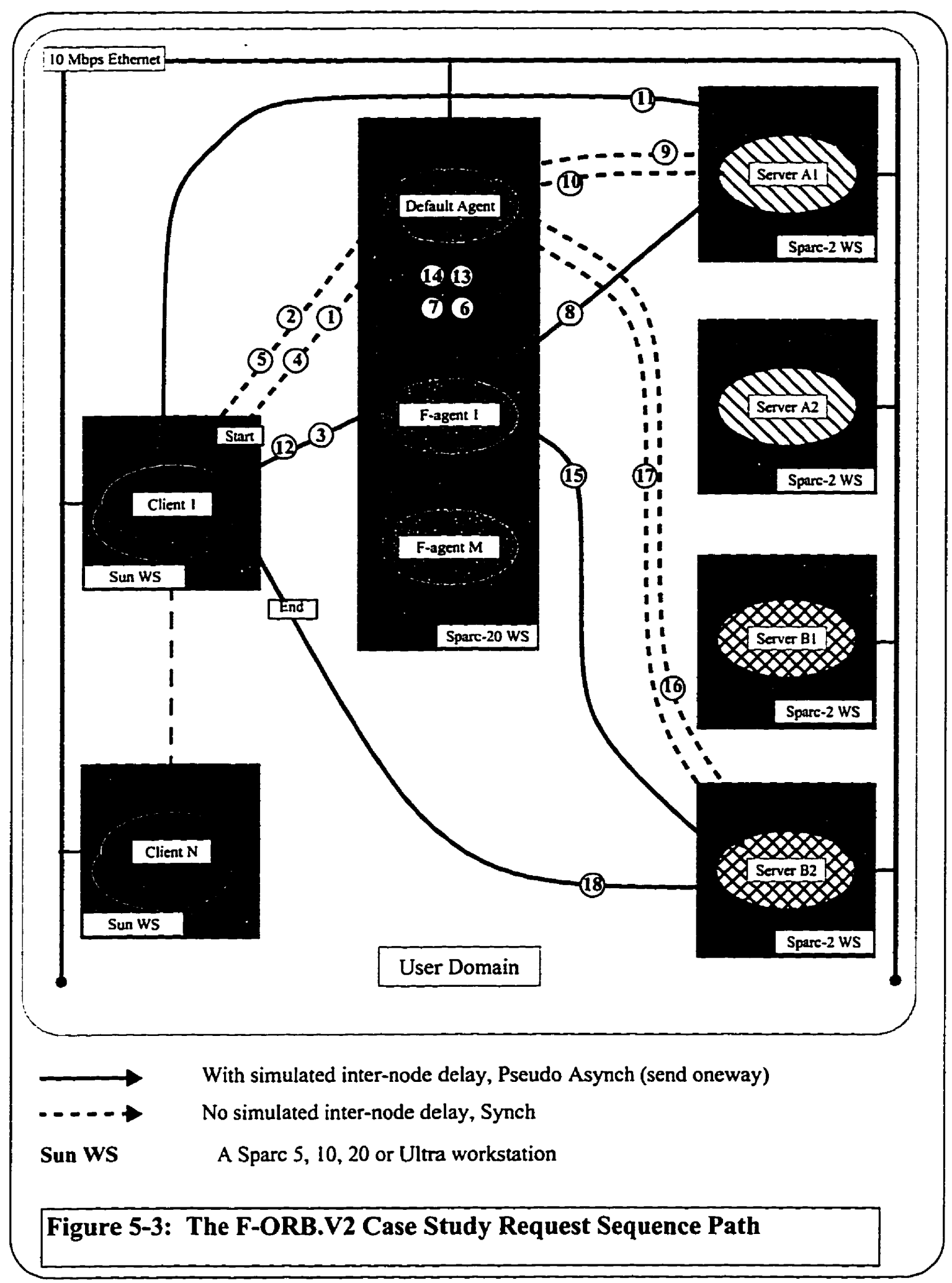


any simulated delays. Let $F$-agent $I$ be the agent, the handle of which is returned.

- Step 3: The client forwards the request to $F$-agentl using ORBeline's send oneway pseudo asynchronous call. The client sleeps before sending to the fagent to simulate the preset network inter-node delay.

- Steps 4-5: The client registers itself with the default agent in order to be accessible to the server processes in the domain. No simulated delays are involved. This is done only once during the lifetime of the experiment.

- Steps 6-7: F-agentl obtains handle for a server of class $A$. Let $A 1$ be the servers handle of which is retumed. This dynamic binding is necessary to maintain load balancing and fault tolerance. No simulated delays are involved

- Step 8: F-agentl uses the handle to relay the request to $A I$ in a pseudo asynchronous mode. The f-agent sleeps before sending to $A l$ to simulate the preset network inter-node delay.

- Step 9-10: In order for $A l$ to return the reply (after processing the request) to the originating client, it must first obtain its handle from the default agent. As discussed earlier in the agent/server interaction in this chapter, $A I$ does not sleep while getting this handle.

- Step 11: Al returns the reply to the originating client using the pseudo asynchronous mode. $A I$ sleeps prior to sending the reply to simulate the preset network inter-node delay.

- Step 12: Since the f-agent handle has been obtained earlier, there is no need for 
a second handle request (binding) from the default agent. Hence the client forwards its request (destined for a class $B$ ) server using a pseudo asynchronous call to F-agent 1 . The client sleeping behavior is identical to that of step3.

- Steps 13-14: F-agent I dynamically obtains a handle for a class $B$ server (for the same reasons stated in steps 6-7). Let $B 2$ be the server, the handle of which is returned. No simulated delays are involved

- Steps 15: $F$-agentI uses the handle to relay the request to $B 2$ in a pseudo asynchronous mode. The agent sleeping behavior is similar to that in step 8 .

- Steps 16-17: In order for B2 to return the reply (after processing it) to the originating client, it must first obtains its handle from the default agent. $B 2$ does not sleep while getting this handle for the same reasons stated in steps (910).

- Step 18: Server $B 2$ retums the reply to the respective client in a pseudo asynchronous mode and thereby ending the client request cycle. B2 sleeps prior to sending the reply to simulate the preset network inter-node delay.

\subsubsection{The Process Planner P-ORB System}

The P-ORB differs from the $\mathrm{H}-\mathrm{ORB}$ architecture because its p-agent is more complex than the default agent as described in Section 4.5 in Chapter 4. The major components are distributed as follows:

a The Client Nodes: The clients are distributed over a pool of available workstations 
as in the previous architecture. Details are in Subsection 5.1.2.1.The client can take advantage of the complex p-agent and send its two requests combined in one message to the p-agent. The p-agent decomposes the request into its simple constituent services (a checking request and a saving request in this case study). Since the design assumes no interdependencies between the two constituent requests, the p-agent invokes both servers in pseudo parallel asynchronous mode (both server are invoked using the send oneway call, however, there is only one inter-node delay involved). Since the clients have very little computational demand on their own nodes (workstations), negligible differences have been observed in the cycle time of the clients that are distributed over a range of heterogeneous nodes.

b - The Server Nodes: This architecture has a total of four server nodes. Each node is a Sparc-2 workstation. As described earlier, there are two classes of CORBA objects(servers), class A and class B. Each class has two instances, hence there is one server process per machine. Moreover, each server process has its own dispatcher (a FIFO queueing process) on the same node working to avoid any deadlocks created by the nature of the pseudo asynchronous message protocol. Figure 5-4 and Figure 5-5 illustrate two examples of deadlocks that may be encountered when no dispatchers are used in the design.

c The Agent Node: This is a Sparc-20 workstation. This node will have the default agent as well as the p-agents. During each experiment, a fixed number of $p$-agents are activated and set ready to receive and process any client request in cooperation with the default agent supplied by ORBeline. As explained earlier in the workload in 
Subsection 5.1.1, the clients, the p-agents, and the servers need to obtain the handle of the sought process to be able to forward the request to it. Having no access to the source code of the default agent has made impossible to modify it to include the functionalities of the p-agent in it. Hence, the co-existence of both the default-agent and the p-agents on the same workstation is necessary to simulate their integration into a complex agent. Hence, the default-agent presence is mandatory in this architecture. Figure 5-6 illustrates the client Request Sequence Path in this architecture.

d - The P-ORB Request Path: The request in this architecture follows a different path in comparison with the previous architectures. The p-agent is the process which decomposes the client's composite request into its constituent services, invokes the respective servers and when all services are performed, it relays back a single coherent reply to the originating client. The path steps that are numbered in Figure 56 are described below. It should be noted that some of the steps include ORBeline's Pseudo Asynchronous (send oneway) calls to the called nodes:

- Steps 1-2: Obtaining the handle for a p-agent from the default agent. Let $\boldsymbol{P}_{\text {- }}$ agentI be the agent, the handle of which is returned. In contrast to the H-ORB architecture, the client does not sleep (no simulated delay) while getting this handle to make its behavior close to the ideal P-ORB description in Chapter 4 in Subsection 4.5.2.1.

- Step 3: The client forwards the request to P-agentI using CORBA's send oneway call.The client sleeps before sending to the p-agent to simulate the 


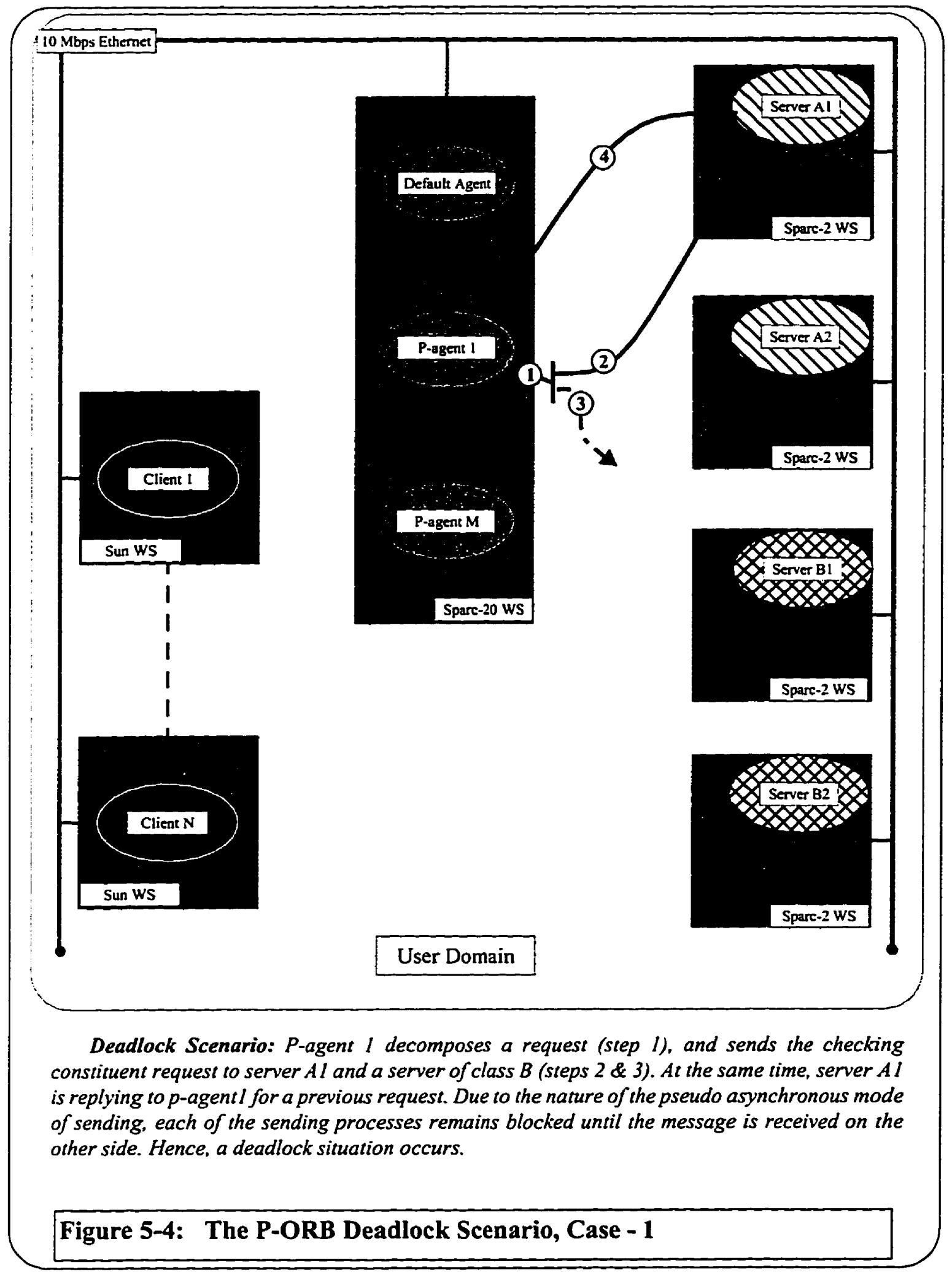




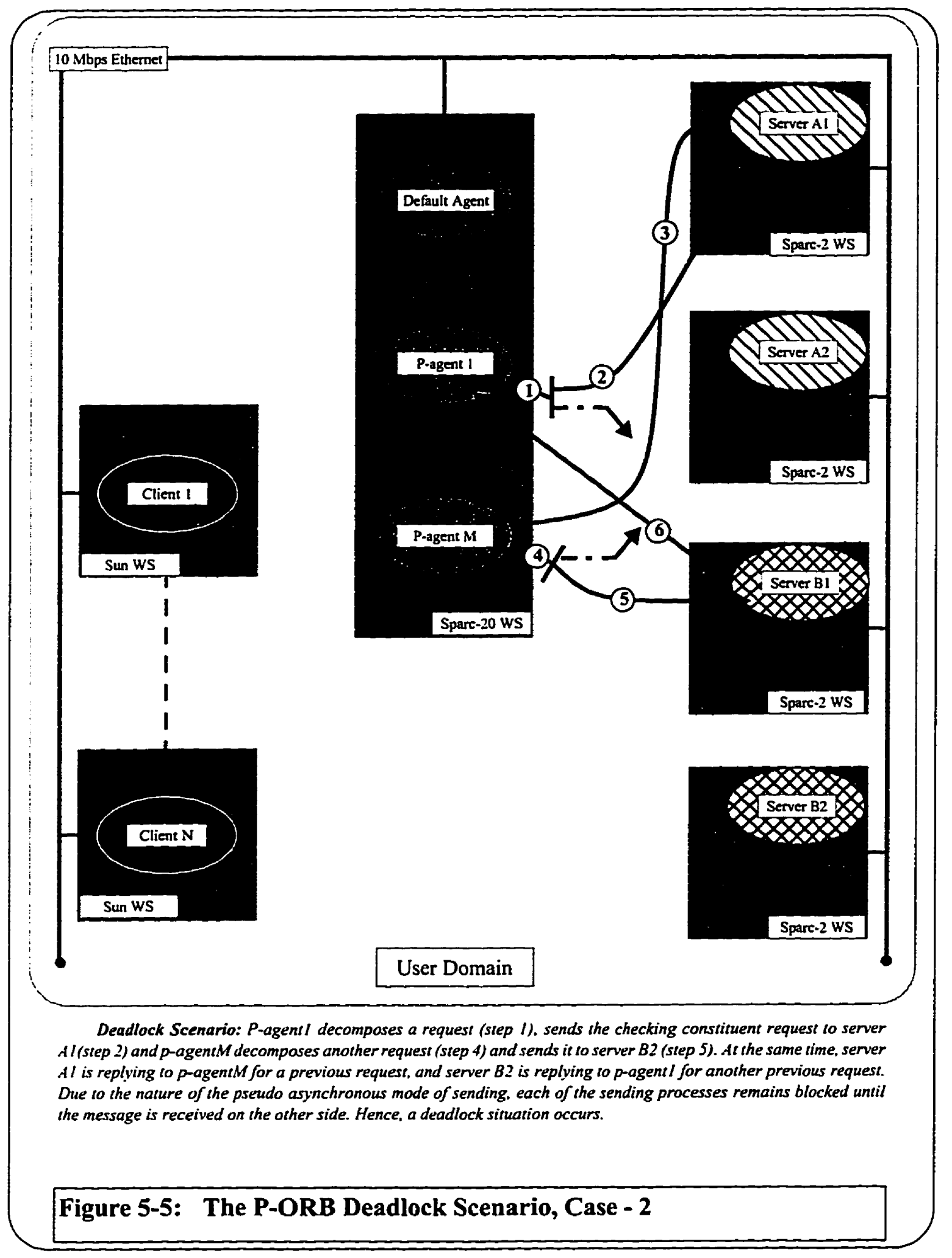




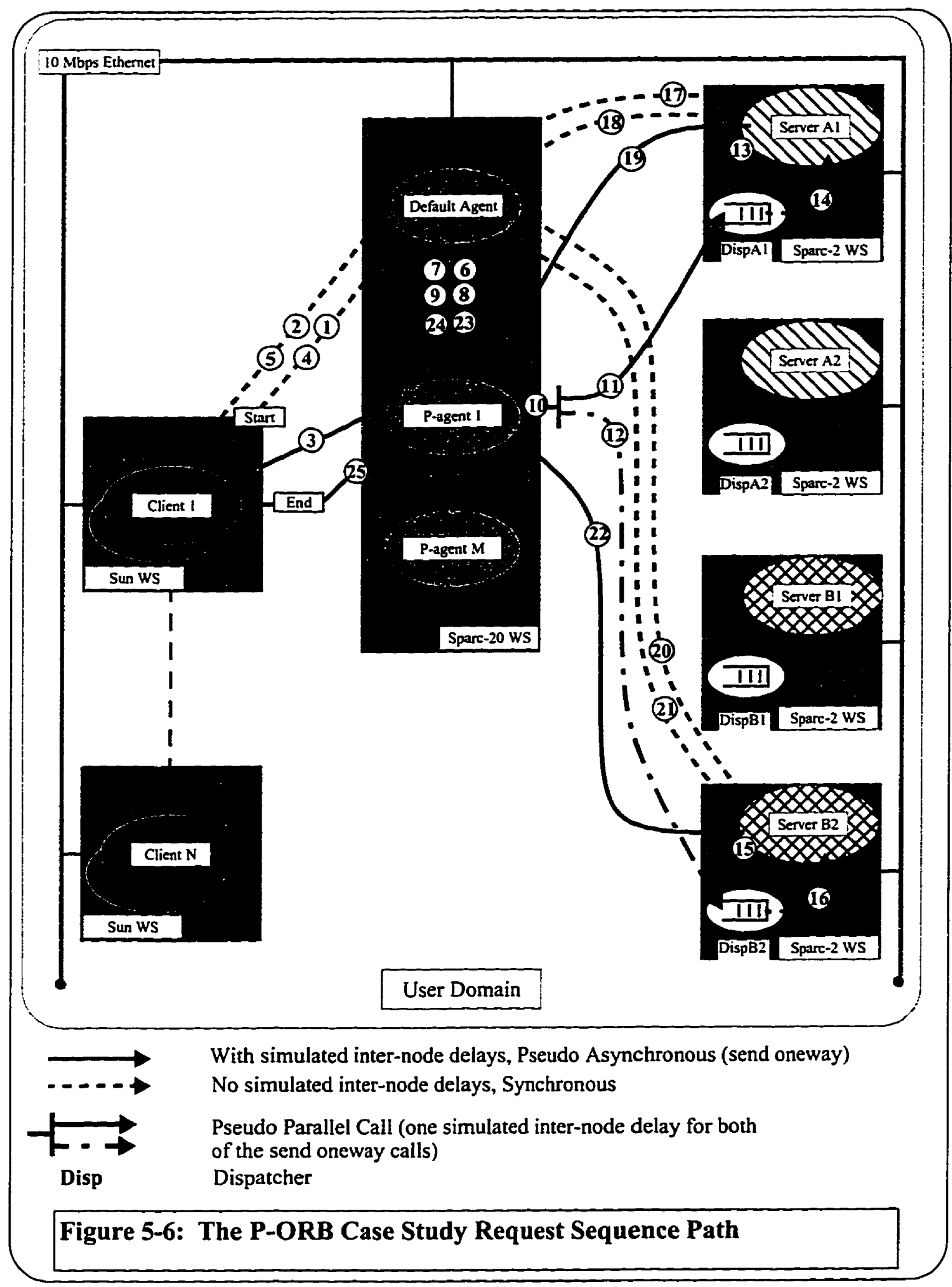


preset inter-node delay.

- Steps 4-5: The client registers itself with the default agent in order to be accessible to the server processes in the domain. No simulated network delays are involved.This is done only once during the lifetime of the experiment.

- Steps 6-7, 8-9: P-agentl decomposes the request into its constituents and obtains a handle for a dispatcher of a server of class $A$ (in $6-7$ ), and a second handle for a dispatcher of a class $B$ server (in 8-9). Let A1, and B2 be the servers whose dispatchers' handles are returned respectively. No simulated delays are involved.

- Step 10-11-12: P-agentl uses these handles to relay the request to the respective servers (dispatchers) in a pseudo parallel asynchronous mode (one simulated inter-node delay for both of the send oneway calls).

- Step 13-14, 15-16: Each server notifies the respective dispatcher (queue manager) that it is ready, and if there is a request waiting in the queue then it is dispatched. To avoid any dead locks, the queue will refrain from dispatching any further requests until the server notifies it again.

- Step 17-18: In order for $A 1$ to retum the reply to $p$-agent1, it must first obtain its handle from the default agent (without simulated delays). These steps can be optional if $A l$ has stored the handle locally from a previous invocation.

- Step 19: $A 1$ returns the reply to the calling p-agent (p-agnetl) in a send oneway mode after sleeping for the preset inter-node delay.

- Step 20-21: In order for $B 2$ to return the reply to the p-agent1, it must first 
obtain its handle from the default agent. These steps can be optional if $B 2$ has stored the handle locally from a previous invocation.

- Step 22: B2 returns the reply to $p$-agentl in a pseudo asynchronous mode after sleeping the preset inter-node delay.

- Step 23-24: In order for P-agentl to return the reply to the originating client, it must first obtain its handle from the default agent (without simulated delay). These steps can be optional if p-agent 1 has stored the handle locally from a previous invocation.

- Step 25: P-agentl packs all constituent replies into a final, single and coherent reply and send it to the originating client, after sleeping the preset inter-node delay. $P$-agent $I$ uses the same pseudo asynchronous mode to return the reply to the respective client. The message reception by the client side indicates the end of the request cycle.

\subsection{Experimental Results}

The three main CORBA-based client/server architectures, the H-ORB, the F-ORBCVI \& V2), and the P-ORB, were implemented. Sets of experiments were run to study the effect of the factors, stated in Subsection 5.1.1, that reflect the characteristics of both the system and the workload. A factor-at-a-time approach was adopted for each experiment. One of the factors was varied while the others were held constant. The results of each experiment is presented as a set of graphs that capture the relationship between one of the performance measures of interest (plotted along the $\mathrm{y}$ axis), and the number of clients $\mathrm{N}$, (plotted along 
the $\mathrm{x}$ axis) with all the other fixed parameters stated in the figure caption. The performance measures of interest are described next.

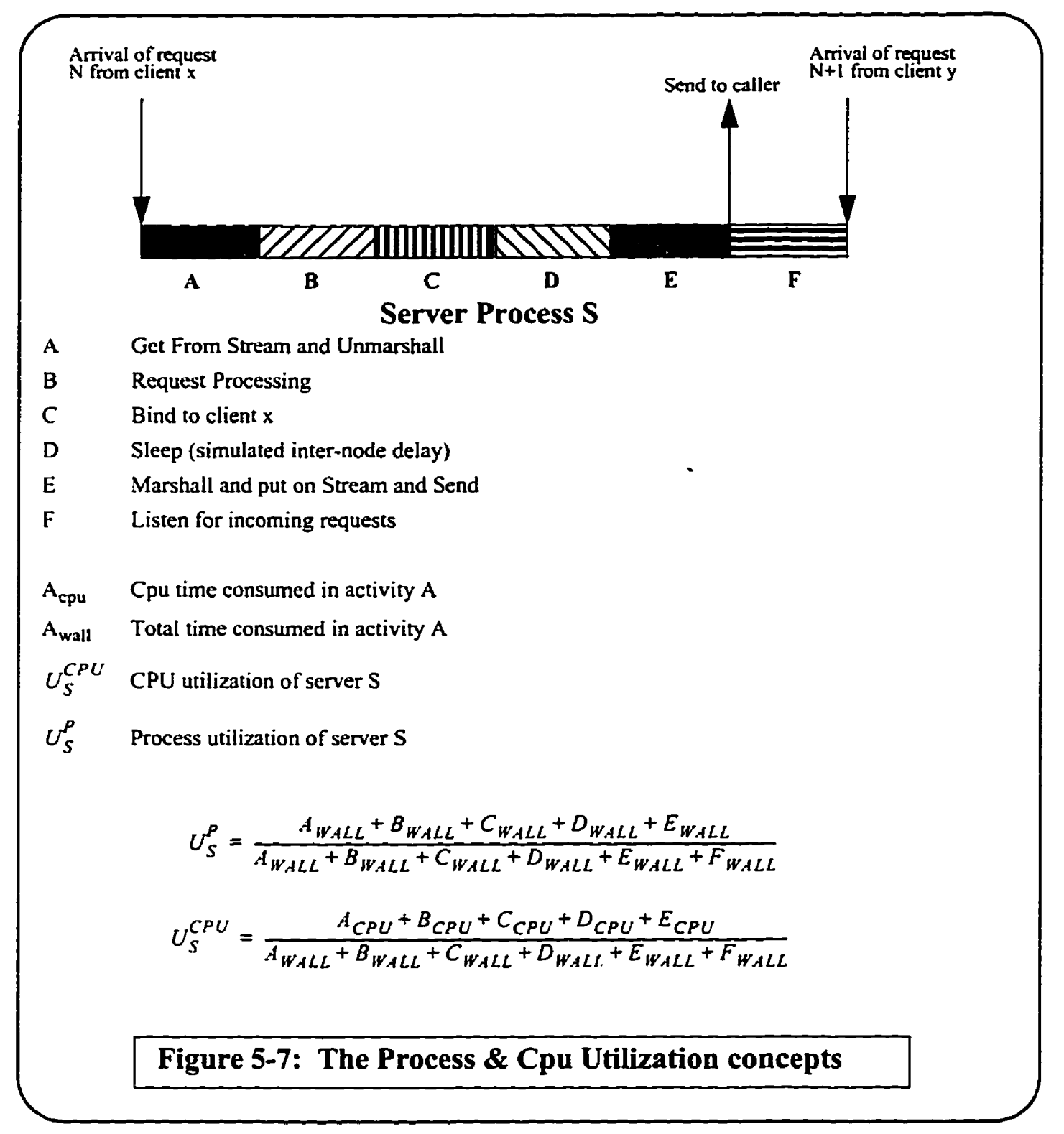

1) Mean Client Response Time (R) in seconds: This is the mean total elapsed time from the instant the client starts its request (including the message parameters initialization, obtaining the handle, marshalling etc.) until the final 
reply arrives and gets unmarshalled from all the servers (in this case study the reply has to be received from the two server classes). The mean client response time is a measure of the latency of the system.

2) Overall Mean System Throughput $(X)$ in client requests per second: This is the average total system throughput which is obtained by the summation of all client's mean throughputs. The throughput is a measure of the capacity of the system. The throughput and response time are related by little's law $(N=X R)$.

3) Process and CPU Utilization $(U)$ : These utilizations are measured to gain more insight into the system performance and to identify the hardware and software bottlenecks. Figure 5-7 illustrates the concepts of both utilizations and how they are calculated in the coming experiments. The wall clock time is used to measure the blocking time of the process as no other means, to our knowledge, exists.

All of the experiments were performed during night time runs to avoid the effect of other jobs on the network. The effect of occasional interference from system processes such as daemons was removed in the post processing of data: the results that were deviant from the others by more than three standards deviations were discarded. The number of client cycles was large enough to produce an interval that is less than $\pm 5 \%$ of the mean at a confidence level of $95 \%$ for the performance measure of interest. The impact of D, L, and $\mathrm{S}_{\mathrm{A}} / \mathrm{S}_{\mathrm{B}}$ on the relative performance of the three architectures is covered in the next subsections. For each factor, the relative performance of the three architectures is studied 
both at low as well as medium to high load levels of the system. The load is increased by increasing the value of $\mathrm{N}$. Two performance attributes, latency and scalability are of interest. At low load, there is no queueing, but the queueing behavior can be effectively captured at medium and high loads. At low load the performance measure of interest is the mean client response time that represents the latency properties of the three architectures. At medium to high load the overall system throughput is used to study the scalability properties of the three architectures.

\subsubsection{Experiment Control and Data Logging}

In order to setup the clients, agents, and servers on different machines and synchronize their data gathering, DECALS [ELG-94](Distributed Experiment Control and Logging System) - a tool used to run and control distributed applications over a network, collect measurements and trace the related data, was used. DECALS reads a set of configuration files (for the system configuration), a list of experiments file - LOE (which specifies the number of different experiments to be conducted) and one or more experiment description file - EXP (which indicates what is to be done for the experiments to be performed). An Experiment, in DECALS terminology, is the allocation of application processes to network nodes, the execution until completion of those application processes and the collection of measurements and trace information from those processes. Once the configuration information has been read, DECALS performs each experiment in turn. For each experiment, DECALS executes and synchronizes all the application processes (clients, agents and servers), and simultaneously runs the collector, a DECALS process used to collect the application's measurements and trace information using a procedural interface 
provided by DECALS libraries. For experiment termination, DECLAS uses a time-out period in addition to the natural death (normal completion) of the experiment. Due to the observed overhead of logging of the various events in each request cycle in the experiments, all measurements per request cycle were stored in data structures local to the respective process. At the end of the experiment, the stored data is processed and the final results are witten to DECALS log file as a single event.

\subsubsection{Elapsed and Cpu Time Measurements}

The source code for all the architectures was instrumented at the appropriate positions of code in order to measure the elapsed and/or the cpu time spent by the calling process during the execution of a specific portion of the source code. A description of the Unix System calls used is provided.

a - gettimeofday 0: This system call gets the system's notion of the current time. The current time is expressed in elapsed seconds and microseconds since 00:00 Universal Coordinated Time, January 1, 1970. The resolution of the system clock is hardware dependent and the time may be updated continuously or in clock ticks [MAN-95]. In all experiments, the elapsed time was measured using this function except for cases where another approach is stated explicitly.

b - gethrtime0: This function returns the current high-resolution real time. Time is expressed as nanoseconds since some arbitrary time in the past. It is not correlated in any way to the time of day, and thus is not subject to resetting, drifting, etc. via oldtime 0 or settimeofday 0 system calls. The hi-res timer is ideally suited to 
performance measurement tasks, where cheap, accurate interval timing is required [MAN-95].

c - ioctl0: This Solaris system call is used with the PIOCUSAGE flag to obtain the cpu time consumed by the calling process. In all the measurements, the cpu time is calculated by adding the user level cpu time and the system call cpu time [MAN-95].

\subsubsection{The Performance of the H-ORB on a Single Workstation}

Studying the performance of the main CORBA components that an H-ORB client request invokes during a complete cycle is important to gain insight into the system behavior. Therefore, the H-ORB architecture was implemented and tested under no network delays (the client, the agent and the servers are located on a single Sparc-20 workstation). The parameters of the experiments are listed in Table 2, and the execution scenario is given in Figure 5-8

Table 2: Parameters of the Local H-ORB Experiments

\begin{tabular}{|l|l|}
\hline \multicolumn{1}{|c|}{ Main Parameters } & \multicolumn{1}{c|}{ Range of values } \\
\hline Number of Clients & $I$ \\
\hline Service Times & $\begin{array}{l}\text { Class A server }=10 \mathrm{~ms} \\
\text { Class B server }=15 \mathrm{~ms}\end{array}$ \\
\hline Inter-Node Delay & NIL \\
\hline Message size & 150 Bytes \\
\hline
\end{tabular}

Both the Cpu and the Elapsed (high resolution timer was used) times are measured for the main functions invoked during the request cycle. The number of clients $\mathrm{N}$ was set to 1 . During each cycle, the invoked client stub functions were measured along with the corresponding functions in the servers' skeletons. A study of Table 3 reveals the fact that 
the binding operation consumes the largest amount of the cpu time used by the client in each cycle.

Table 3: Cpu and Elapsed Time of the Invoked Functions in a Client Request Cycle in a Local H-ORB Environment

\begin{tabular}{|c|c|c|c|}
\hline Measured & Client Process & Class A Server & Class B server \\
\hline (sec) & 1 client & 1 client & 1 client \\
\hline Bind $C_{p u C h k}$ & 0.004023 & - & - \\
\hline BindWallChk & 0.010858 & - & - \\
\hline MarshCpuChk & 0.000237 & 0.000250 & - \\
\hline MarshWallChk & 0.000502 & 0.000384 & - \\
\hline Invoke CpuChk & 0.000837 & 0.010656 & - \\
\hline InvokeWallChk & 0.015299 & 0.011251 & - \\
\hline UmarshCpuChk & 0.000356 & 0.000373 & - \\
\hline UmarshWallChk & 0.000709 & 0.000477 & - \\
\hline RlsCpuChk & 0.000805 & - & - \\
\hline RlsWallChk & 0.002202 & - & - \\
\hline BindCpuSav & 0.004004 & - & - \\
\hline BindWallSav & $0.01087 \mathrm{l}$ & - & - \\
\hline MarshCpuSav & 0.000245 & - & 0.000254 \\
\hline MarshWallSav & 0.000604 & - & 0.000466 \\
\hline Invoke CpuSav & 0.000875 & - & 0.015805 \\
\hline InvokeWallSav & 0.022627 & - & 0.018139 \\
\hline UmarshCpuSav & 0.000348 & - & 0.000374 \\
\hline UmarshWallSav & 0.000808 & - & 0.000495 \\
\hline RIsCPuSav & 0.000768 & - & - \\
\hline RlsWallSav & 0.001784 & - & - \\
\hline CycleTime & 0.062835 & - & - \\
\hline
\end{tabular}




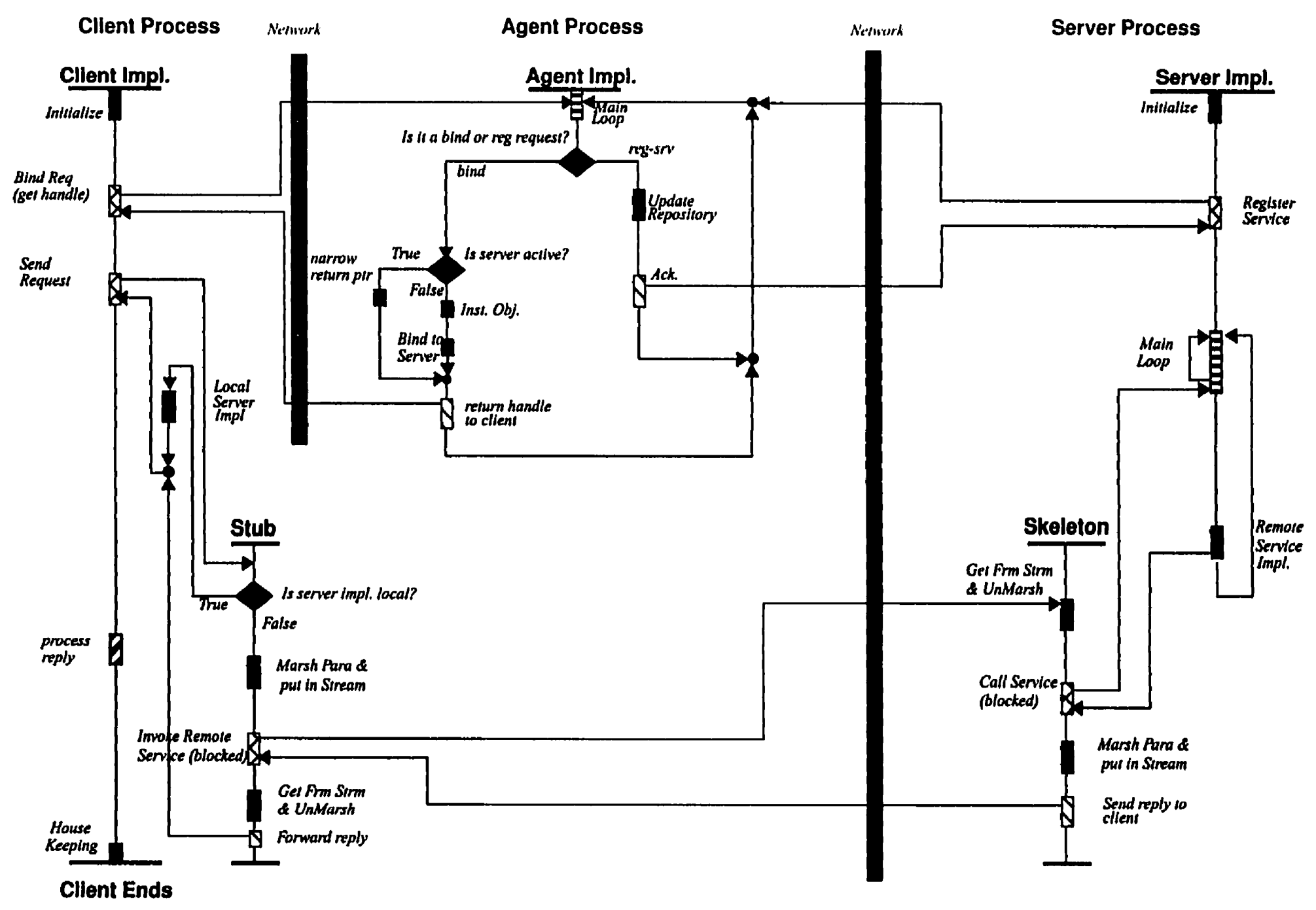

Figure 5-8: The H-ORB Execution Scenario using Client Static

Invocation and Persistent Servers 


\subsubsection{The Effect of Message Size}

Client requests are expected to vary in the size of the message sent to the sought servers depending on the type of the request and the nature of the service provided. This set of experiments is designed to investigate the relative performance of the three main architectures as the message size $\mathrm{L}$ increases. Table 4 presents the various parameters used in this set of experiments, with the variable parameters in italic fonts.

Table 4: Parameters of Message Size Experiments

\begin{tabular}{|l|l|}
\hline \multicolumn{1}{|c|}{ Main Parameters } & \multicolumn{1}{|c|}{ Range of values } \\
\hline Number of Clients & $1,2,4,816,24$ \\
\hline Service Times & $\begin{array}{l}\text { Class A server }=10 \mathrm{~ms} \\
\text { Class B server }=15 \mathrm{~ms}\end{array}$ \\
\hline Inter-Node Delay & $200 \mathrm{~ms}$ \\
\hline Message size & $4800,9600,19200$ Bytes \\
\hline $\begin{array}{l}\text { Degree of Cloning: } \\
\text { H-ORB Arch. } \\
\begin{array}{l}\text { F-ORB Arch. } \\
\text { P-ORB Arch. }\end{array}\end{array}$ & $\begin{array}{l}\text { Default Agent }=1 \\
\text { Default Agent }=1, \text { F-agents }=1,4,8 \\
\text { Default Agent }=1, \text { P-agents }=1,4,8\end{array}$ \\
\hline
\end{tabular}

For each value of $\mathrm{L}$ and a given degree of cloning, the number of clients $\mathrm{N}$ is increased from 1 to 24. As the number of bytes in each message increases, the marshalling/ unmarshalling overhead and the underlying ORB core utilization increases. The messages exchanged between the agent and the client are small in case of the H-ORB because they contain a request and the address of a server. The set of graphs shown in this section presents the results obtained when the message size was set at 4800 bytes under various degrees of cloning. Results for the other message sizes can be found in Appendix A Figures A-I to A- 
29. F-ORB.V1 and V2 architectures were run over all the ranges of message sizes and degrees of cloning. Both exhibited comparable results (with in $\pm 5 \%$ difference). However, due to the large number of graphs, only one case study of the F-ORB.V2 (with no-cloning and $\mathrm{L}=4800$ Bytes) is presented in Appendix A in Figures A-1 to A-3. It should be noted that unless the version number is explicitly stated all data for the F-ORB presented in this chapter correspond to the performance of the F-ORB.V1.

\subsubsection{No Cloning (Single Agent) Architectures:}

Message Size - 4800 Bytes: The influence of the message size as the number of clients increases on the mean client response time and the corresponding overall mean system throughput is investigated at low, medium, and high load conditions.

Table 5: Effect of $L$ on Latency at $N=1\left(D=200 \mathrm{~ms}, S_{A} / S_{B}=10 \mathrm{~ms} / 15 \mathrm{~ms}\right.$, no Cloning)

\begin{tabular}{|c|c|c|c|}
\hline L (bytes) & H-ORB (sec) & F-ORB (sec) & P-ORB (sec) \\
\hline 4800 & 1.7212 & 1.3142 & 0.9015 \\
\hline 9600 & 1.7425 & 1.3448 & 0.9030 \\
\hline 19200 & 1.7933 & 1.4141 & 0.9125 \\
\hline
\end{tabular}

\section{Low Load Conditions:}

As captured in Table 5, Figure 5-9, and Figure 5-10 at $\mathrm{N}=1$ the P-ORB demonstrates the best latency followed by the F-ORB and then the H-ORB. Since there is no queueing, the latency obtained at $\mathrm{N}=1$ is determined primarily by the number of messages exchanged per client cycle. The H-ORB requires four messages for interacting with any one server: the first two correspond to the communication between the H-ORB agent and the client 


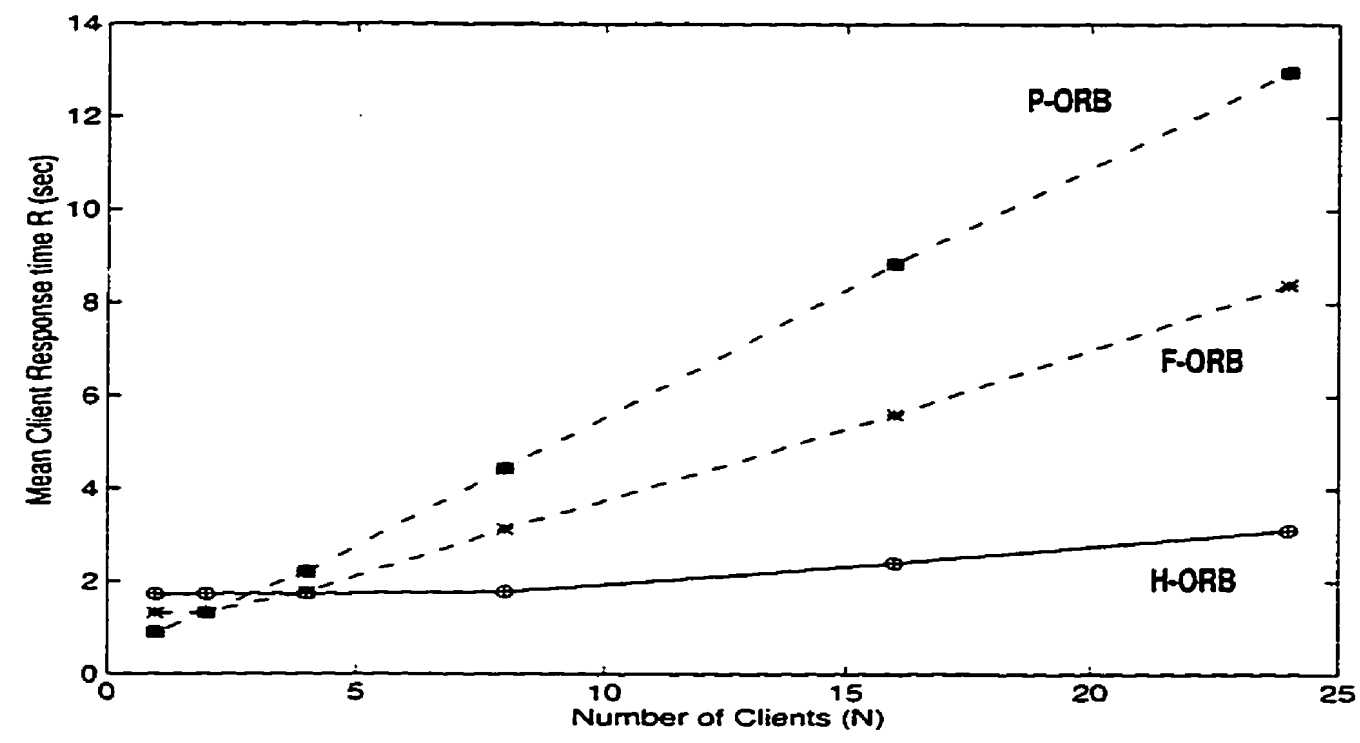

Figure 5-9: The Response Time of the H-ORB, the F-ORB and the P-ORB with $L=4800$ Bytes, and no Cloning.

Degree of Cloning $=1, D=200 \mathrm{~ms}, L=4800$ Bytes, $S_{A}=10 \mathrm{~ms}, S_{B}=15 \mathrm{~ms}$

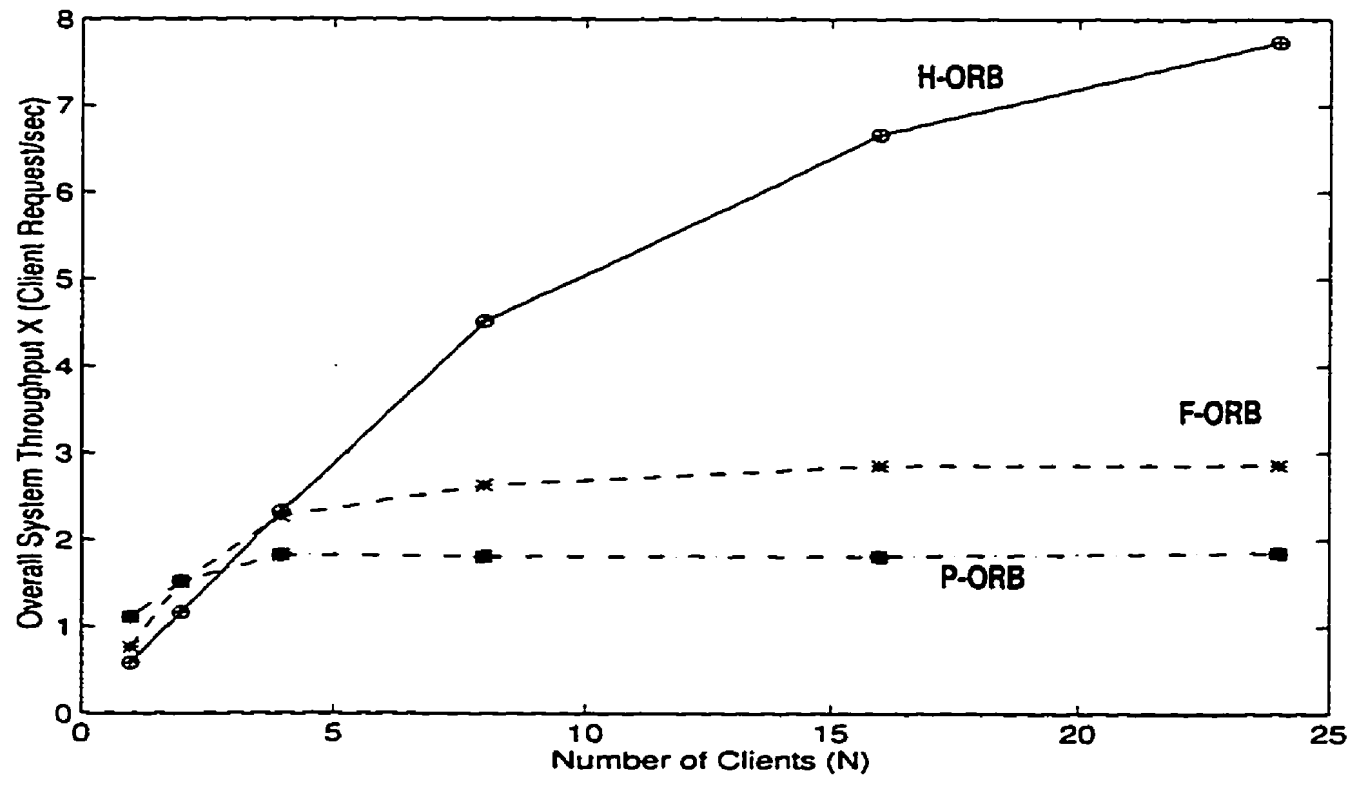

Figure 5-10: The Throughput of the H-ORB, the F-ORB and the P-

$O R B$ with $L=4800$ Bytes, and no Cloning.

Degree of Cloning $=1, D=200 \mathrm{~ms}, \mathrm{~L}=4800$ Bytes, $S_{A}=10 \mathrm{~ms}, S_{B}=15 \mathrm{~ms}$ 


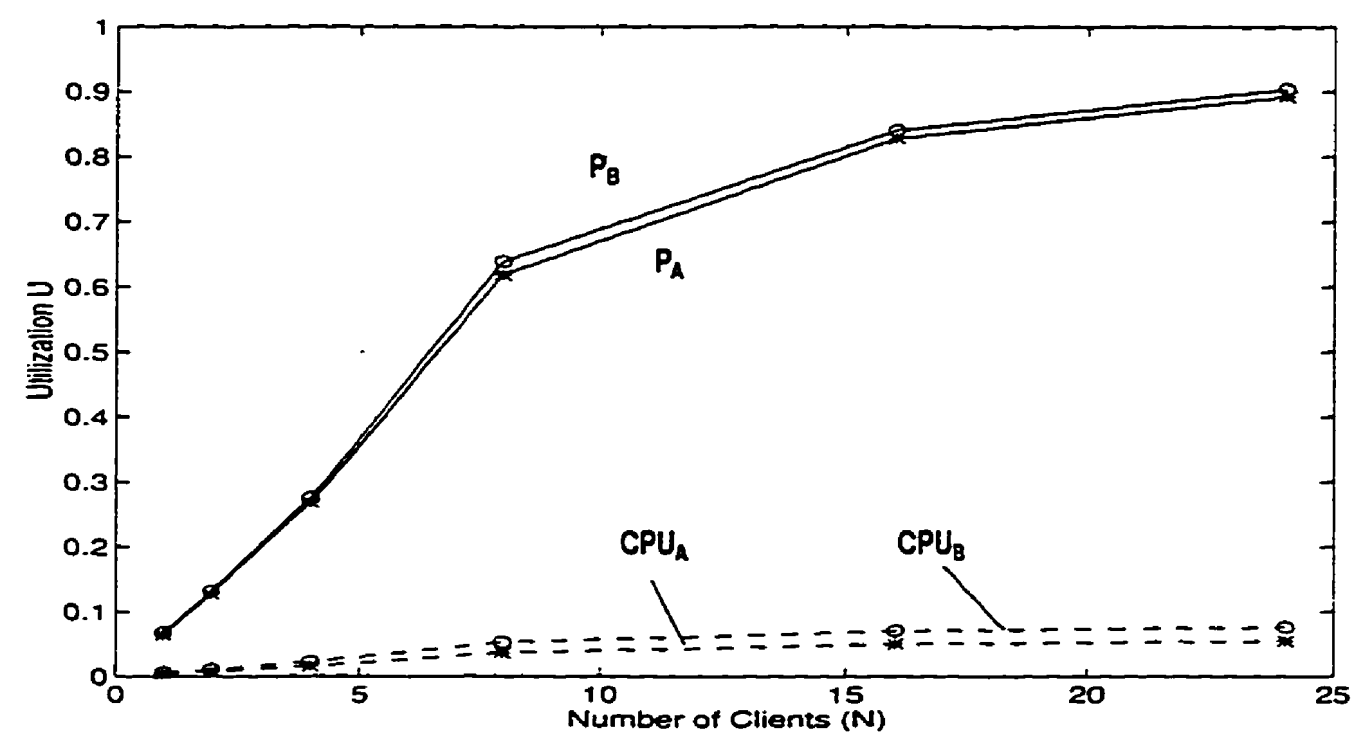

Figure 5-11: Cpu and Process Utilizations of the H-ORB with $L=4800$ Bytes and No Cloning. $P_{A}=$ Process Util. of Server $A, C P U_{A}=C p u$ Util of Server $A$. Degree of Cloning $=1, D=200 \mathrm{~ms}, L=4800$ Bytes, $S_{A}=10 \mathrm{~ms}, S_{B}=15 \mathrm{~ms}$

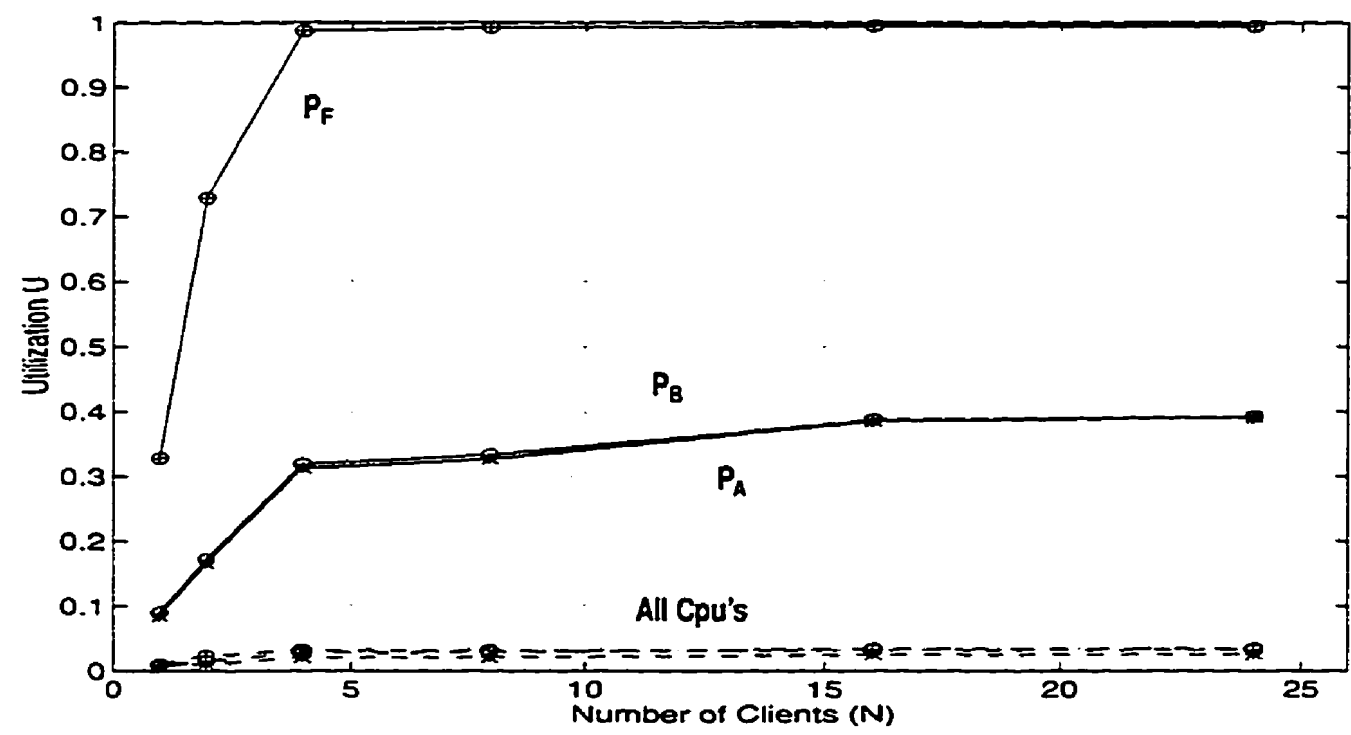

Figure 5-12: Cpu \&Process Utilizations of the F-ORB with $L=4800$ Bytes and No Cloning.

$P_{A}=$ Process Util. of Server $A, C P U_{A}=C p u$ Util of Server $A, P_{F}=$ Process Util. of f-agent. Degree of Cloning $=1, D=200 \mathrm{~ms}, L=4800$ Bytes, $S_{A}=10 \mathrm{~ms}, S_{B}=15 \mathrm{~ms}$ 


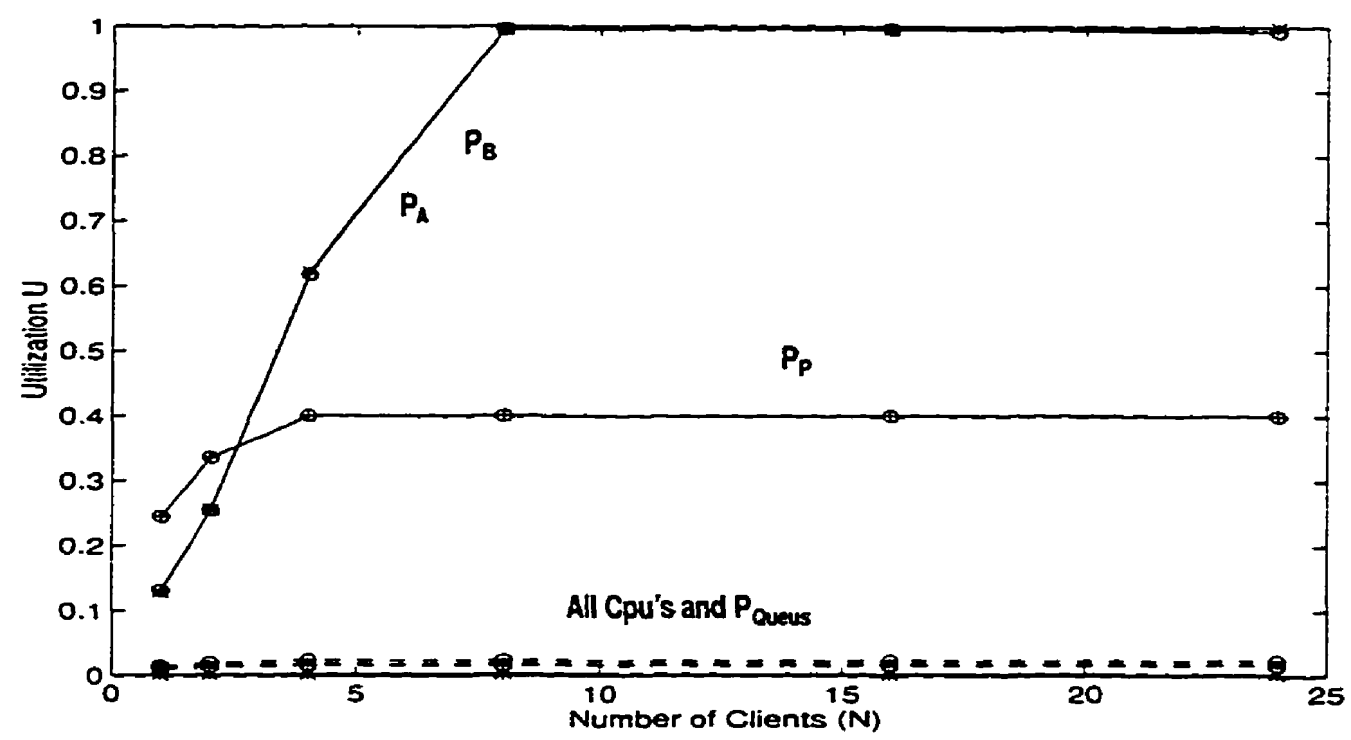

Figure 5-13: Cpu \&Process Utilizations of the P-ORB with $L=4800$ Bytes and No Cloning.

$P_{A}=$ Process Util. of Server $A, C P U_{A}=C p u$ Util of Server $A, P_{p}=$ Process Util. of p-agent.

$P$ Queues $=$ Process Util. of dispatcher, Degree of Cloning $=1, D=200 \mathrm{~ms}, L=4800$ Bytes, $\mathrm{S}_{\mathrm{A}}=10 \mathrm{~ms}, \mathrm{~S}_{\mathrm{B}}=15 \mathrm{~ms}$

whereas the next two result from the communication between the client and server (refer to Figure 5-1 and Subsection 5.1.2.1 for details). The F-ORB requires only three messages per server invocation: a message between the client and the F-ORB agent, a message between the agent and the server and the reply message from the server. Since there are two service requests in a cycle we get eight messages and six messages per cycle for the H-ORB and F-ORB respectively (see Figure 5-2, Figure 5-3, and Subsection 5.1.2.2). As a result FORB demonstrates a better latency in comparison to $H-O R B$ at $N=1$. The P-ORB on the other hand requires six messages per cycles: two between the client and the P-ORB agent and two between agent and server for each server interaction (consult Figure 5-6 and Subsection 5.1.2.3). Although the total number of messages is the same as in the case of the F-ORB, the P-ORB demonstrates a smaller latency because of the concurrency in 
execution: both the servers execute concurrently in the P-ORB architecture whereas they are invoked in sequence for the two other architectures. Moreover, due to the pseudo parallel communication calls (one simulated delay for both calls) initiated by the p-agent, the number of inter-node delays encountered by the request is reduced.

\section{Medium and High Load Conditions:}

Except for small values of $\mathrm{N}$ (fewer than 4), the H-ORB demonstrates a much superior performance in comparison to the two other architectures for medium to high load. The primary reason for this is a phenomenon called software bottlenecks. A performance bottleneck is the hardware device or a software process that limits the growth in system throughput in response to an increase in the number of clients. The bottleneck device is that device which saturates first in response to a growth in the workload. Conventional time sharing computer systems are characterized by bottlenecks that are hardware devices such as a CPU or disk. Software bottlenecks occur on client server systems [NEI-95] [FON-89] due to the synchronous nature of the send-wait-reply mechanism used in client-server interaction. With such a message passing mechanism, a process after sending a message to another process waits until the message is received. Thus it may be utilized $100 \%$ of the time in terms of executing and waiting for message delivery although the CPU on which it executes is under utilized. As shown in Figure 5-12 the F-ORB agent process, f-agent, saturates at $\mathrm{N}=8$ whereas its $\mathrm{CPU}$ utilization is under $10 \%$. Although the F-ORB requires a smaller number of messages in comparison to the H-ORB it spends more time in waiting to receive and send messages. This is due to the fact that both during the reception as well as forwarding of the message the F-ORB agent spends some time during which the ORB 
performs a number of internal operations including marshaling and unmarshalling of data. Moreover, due to the nature of ORBeline's send oneway the sender remains blocked until the message is picked up (or queued in) on the receiver side. Delays increase with the increase in message size and the number of senders. Additional waiting periods result from the simulated inter-node delays as well. Although the inter-node delays are the same for each message the lengths of messages handled by the H-ORB agent on the other hand are small and correspondingly the processing times spent by the ORB are also small. As a result the F-ORB agent gives rise to a software bottleneck whereas this is not so in case of the H-ORB. As shown in Figure 5-10 the H-ORB architecture is not saturated at $\mathrm{N}=24$ indicating the absence of a fully utilized device or process. This is also confirmed by Figure 5-11 in which both the server processes are below saturation. Note that since the agent process in case of the H-ORB is the default agent inside the ORBeline product we could not measure its utilization. However the non-zero slope of throughput curve at $\mathrm{N}=24$ in Figure 5-10 indicates that the default agent is not saturated. The software bottleneck in case of the P-ORB are the server processes. As shown in Figure 5-13 the server processes are heavily utilized with the underlying CPU utilized under $10 \%$. The P-ORB agent process, p-agent, demonstrates a utilization of $40 \%$ in comparison. This is due to the existence of a dispatch process with each server. As described earlier in this chapter, these dispatchers are necessary to prevent any deadlocks in the system as well as alleviating some of the waiting burden on the P-ORB agent by enqueueing the incoming message as soon as it arrives thereby freeing the agent and making it available for further requests/responses. Both the P-ORB agent process as well as the server processes wait after the sending of a message. 
The waiting time experienced by the agent process per interaction with a client or server is smaller on an average in comparison to that experienced by a server. The agent waits until the message is enqueued at a specific server dispatcher or received by the originating client whereas the server may have to wait if the agent is waiting for any of the other servers or for the respective client to receive a message or the agent itself could be busy updating the status of composite request stored internally (checking the local data base to find out whether there is a pending reply from a server for this request or not). Moreover the server execution time is also higher than that of the agent. As a result the server process saturates prior to the agent.

Message Size - 9600 Bytes: Similar behavioral patterns were observed as shown in Figure A-4 to Figure A-8 in Appendix A.

Message Size-19200 Bytes: Similar behavioral patterns to the previous case studies of were also observed as shown in Figure A-9 to Figure A-13 in Appendix A.

Table 6: Throughput at $\mathrm{N}=24\left(\mathrm{D}=200 \mathrm{~ms}, \mathrm{~S}_{\mathrm{A}} / \mathrm{S}_{\mathrm{B}}=10 \mathrm{~ms} / 15 \mathrm{~ms}\right.$, no Cloning)

\begin{tabular}{|c|c|c|c|}
\hline $\begin{array}{c}\mathbf{L} \\
\text { (Bytes) }\end{array}$ & $\begin{array}{c}\text { H-ORB } \\
\text { (Client Requestsec) }\end{array}$ & $\begin{array}{c}\text { F-ORB } \\
\text { (Client Request/sec) }\end{array}$ & $\begin{array}{c}\text { P-ORB } \\
\text { (Client Request/sec) }\end{array}$ \\
\hline 4800 & 7.7356 & 2.8625 & 1.8524 \\
\hline 9600 & 7.4533 & 2.5935 & 1.7938 \\
\hline 19200 & 6.8688 & 2.4378 & 1.7592 \\
\hline
\end{tabular}

In summary, the effect of message size on the maximum throughput observed at $\mathrm{N}=24$ is displayed in Table 6 for the three different architectures. As expected the throughput achieved with $\mathrm{N}=24$ deteriorates with an increase in message size for any given architecture. This is because more work needs to be performed per client cycle with larger 
messages. However, the relative performances of the architectures do not change at higher values of $L$.

\subsubsection{Four-Degree Cloned Architectures:}

To alleviate the performance problems with software bottlenecks encountered in the previous subsection, cloning of the agent process is used. A clone of a process is its copy that shares a message queue with its parent. Thus, with a set of process clones one process can process an outstanding message when the others are busy. Moreover, since the H-ORB uses the ORBeline agent, we did not have any control over the agent process. Since the agent in the H-ORB is not sanurated (form the analysis of the no-cloning study) cloning of the default agent used in the H-ORB is not required. Therefore, it will maintain its nocloning status and the issue of cloning is applied only to the F-ORB and P-ORB. By cloning the agent process the waiting time for a server is reduced: even though one agent process is tied up in an interaction another free agent process can interact with a responding server.

Hence, there will be four $f$-agents and four p-agents in each of the respective architectures in addition to the default agent supplied by ORBeline. In the implementation of the F-ORB and the P-ORB, the Unix forkO system call was used to create the agent clones. Multithreading the agents was unsuccessful due to the fact that the available ORBeline product was single threaded and would not recognize any thread initiated call.

Message Size - 4800 Bytes: Figure 5-14 and Figure 5-15 show the mean client response time and the corresponding system throughput of the three architectures respectively, under 


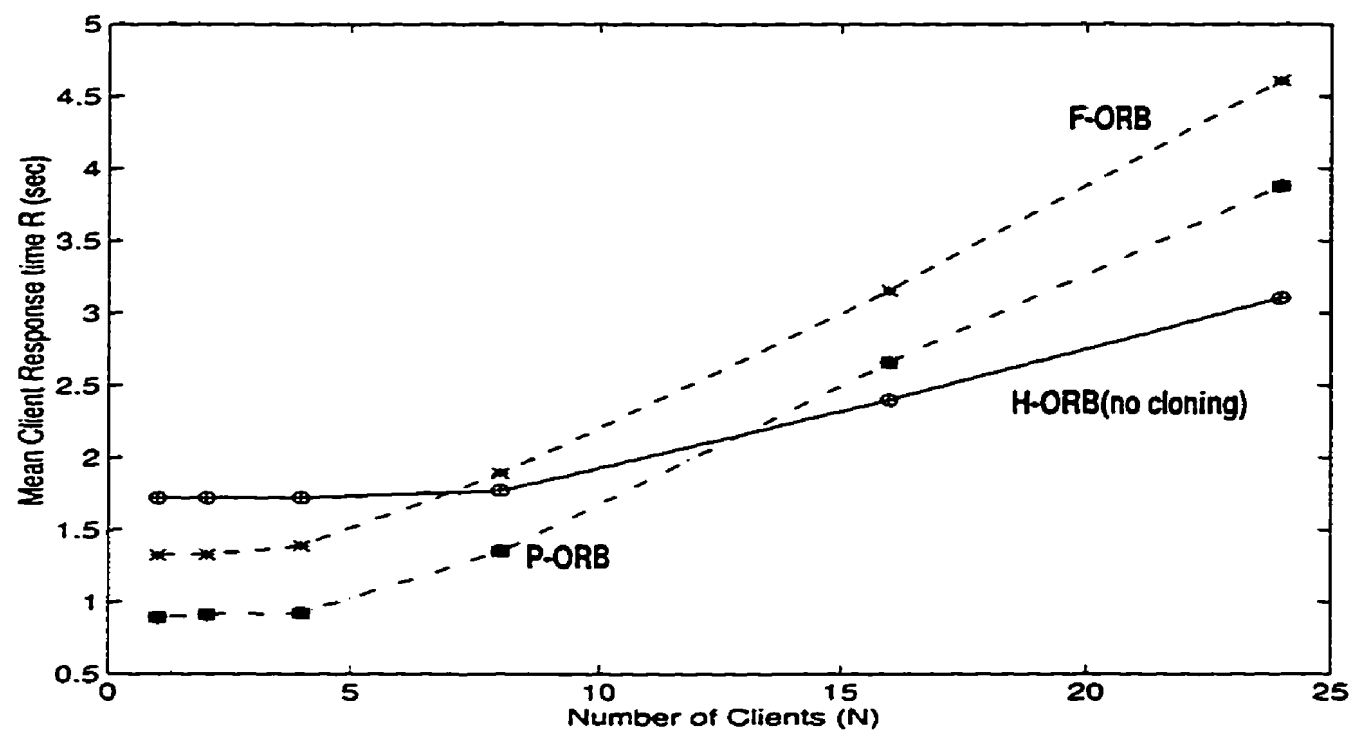

Figure 5-14: The Response Time of the H-ORB, the F-ORB and the PORB with $L=4800$ Bytes, and 4 Cloning Degrees.

Degree of Cloning $=4, D=200 \mathrm{~ms}, L=4800$ Bytes, $S_{A}=10 \mathrm{~ms}, S_{B}=15 \mathrm{~ms}$

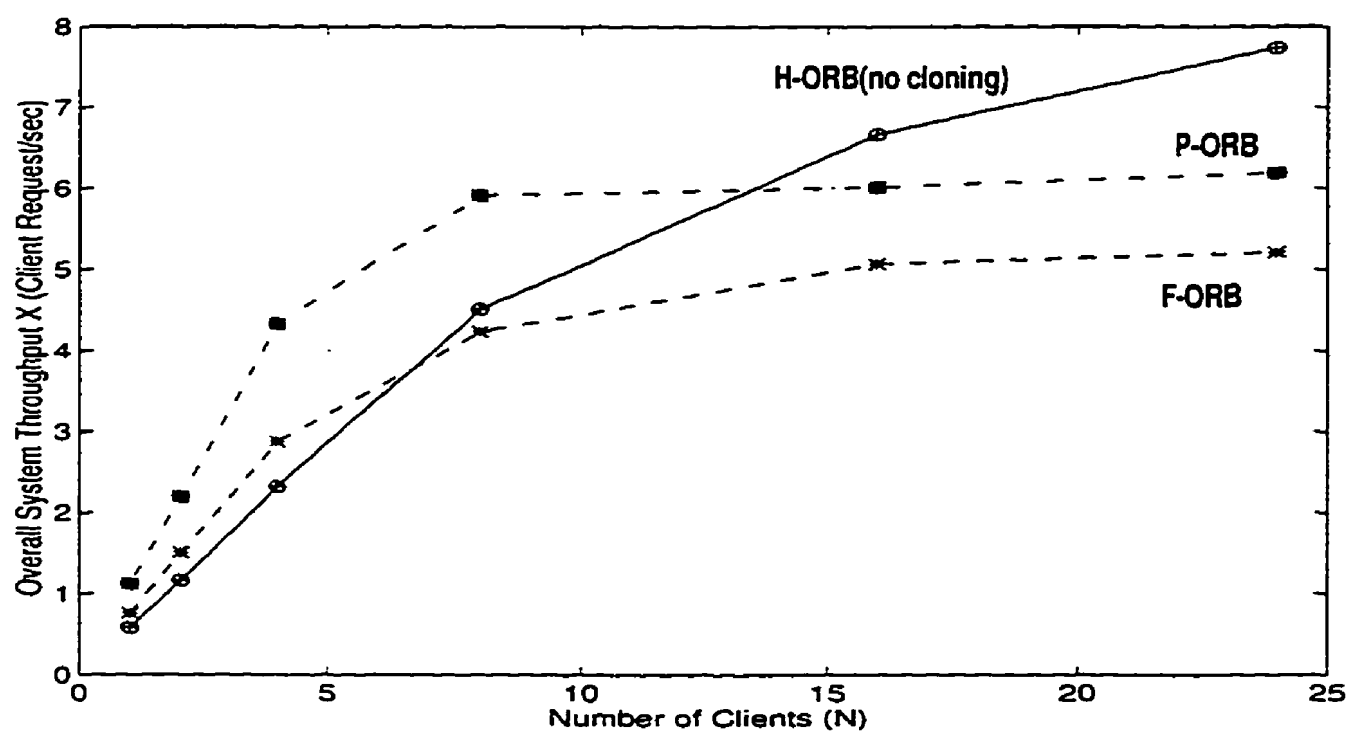

Figure 5-15: The Throughput of the H-ORB, the F-ORB and the PORB with $L=4800$ Bytes, and 4 Cloning Degrees

Degree of Cloning $=4, D=200 \mathrm{~ms}, L=4800$ Bytes, $S_{A}=10 \mathrm{~ms}, S_{B}=15 \mathrm{~ms}$ 
a cloning degree of 4 . As can be observed from the figures and Table 7, the cloning has significantly improved the overall system throughput for the F-ORB and the P-ORB architectures due to the load distribution on the four agents and the consequent reduction of the queueing time at the agents for all incoming requests/replies. Also, as a result of this improvement in performance, the crossover point of the H-ORB with the other two architectures has been shifted to higher values of $\mathrm{N}$.

Table 7: Effect of $L$ on Latency at $N=1\left(D=200 \mathrm{~ms}, S_{A} / S_{B}=10 \mathrm{~ms} / 15 \mathrm{~ms}, 4\right.$ Cloning Degrees)

\begin{tabular}{|c|c|c|c|}
\hline L (bytes) & $\begin{array}{c}\text { H-ORB (sec) } \\
\text { (no Cloning) }\end{array}$ & F-ORB (sec) & P-ORB (sec) \\
\hline 4800 & 1.7212 & 1.3241 & 0.8918 \\
\hline 9600 & 1.7425 & 1.3463 & 0.9017 \\
\hline 19200 & 1.7933 & 1.4232 & 0.9129 \\
\hline
\end{tabular}

The utilization graphs of the F-ORB and the P-ORB are shown in Figure 5-16 and Figure 5-17 respectively.

Message Size - 9600 Bytes: Similar behavioral patterns were observed as shown in Figure A-14 to Figure 5-17 in Appendix A.

Message Size - 19200 Bytes: Similar behavioral patterns to the previous case studies of were also observed as shown in Figure A-18 to Figure A-21 in Appendix A. 


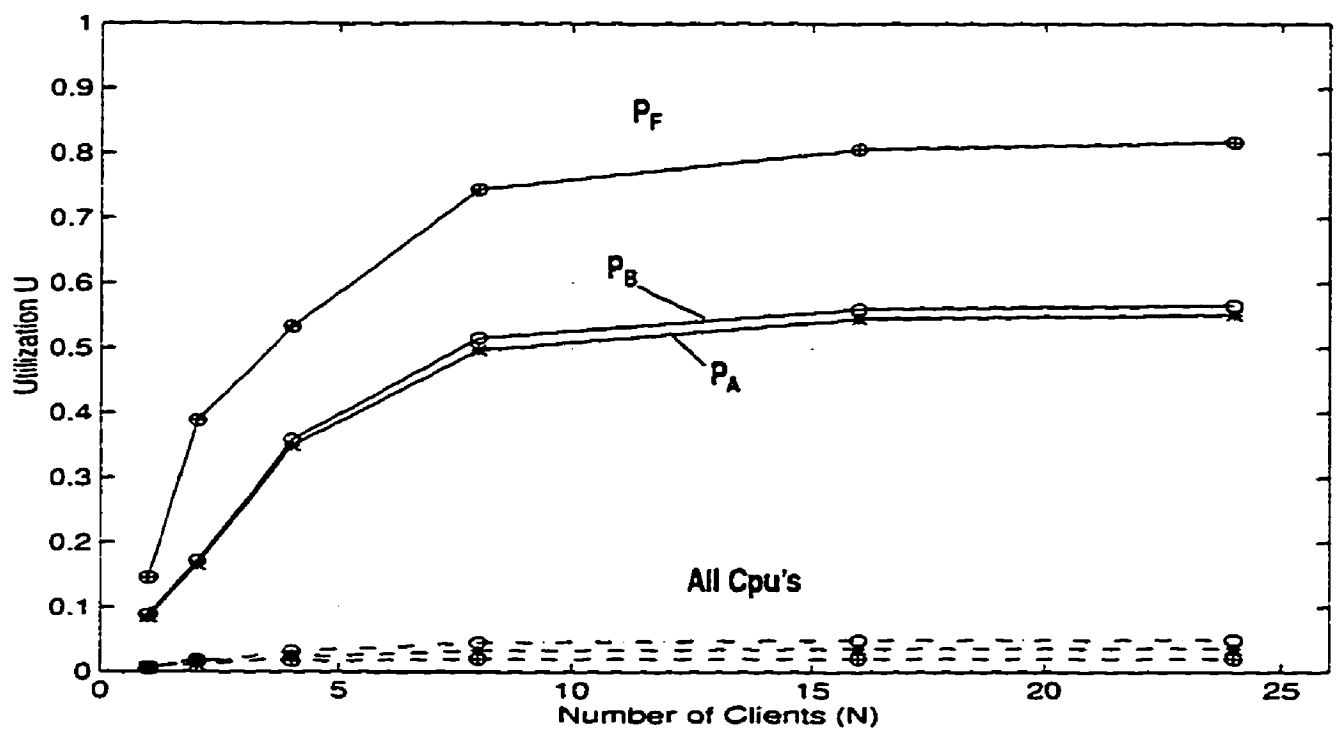

Figure 5-16: Cpu \&Process Utilizations of the F-ORB with $L=4800$ Bytes and 4 Cloning Degrees.

$P_{A}=$ Process Util. of Server $A, C P U_{A}=C p u$ Util of Server $A . P_{F}=$ Process Util. of $f$-agent. Degree of Cloning $=4, D=200 \mathrm{~ms}, L=4800$ Bytes, $S_{A}=10 \mathrm{~ms}, S_{B}=15 \mathrm{~ms}$

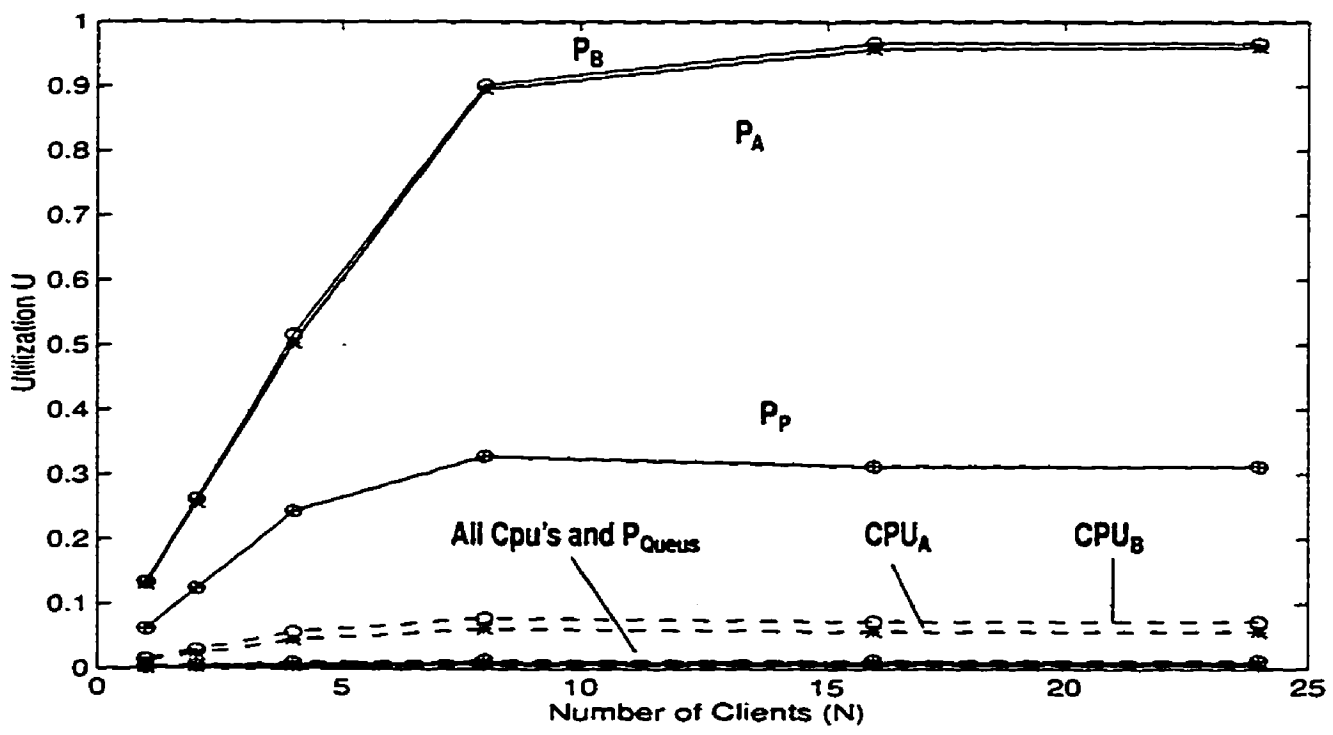

Figure 5-17: Cpu \&Process Utilizations of the P-ORB with $L=4800$ Bytes and 4 Cloning Degrees.

$P_{A}=$ Process Util. of Server $A, C P U_{A}=C p u$ Util of Server A.P $P=$ Process Util. of p-agent. $P_{Q u e u e s}=$ Process Util. of dispatcher, Degree of Cloning $=4, D=200 \mathrm{~ms}, L=4800$ Bytes, $S_{A}=10 \mathrm{~ms}, S_{B}=15 \mathrm{~ms}$ 
Table 8: Throughput at $N=24\left(D=200 \mathrm{~ms}, S_{A} / S_{B}=10 \mathrm{~ms} / 15 \mathrm{~ms}, 4\right.$ Cloning Degrees)

\begin{tabular}{|c|c|c|c|}
\hline $\begin{array}{c}\mathrm{L} \\
\text { (Byres) }\end{array}$ & $\begin{array}{c}\text { H-ORB } \\
\text { (Client Request/sec) }\end{array}$ & $\begin{array}{c}\text { F-ORB V1 } \\
\text { (Client Reques/sec) }\end{array}$ & $\begin{array}{c}\text { P-ORB } \\
\text { (Client Request/sec) }\end{array}$ \\
\hline 4800 & 7.7356 & 5.2095 & 6.1889 \\
\hline 9600 & 7.4533 & 4.9309 & 5.8226 \\
\hline 19200 & 6.8688 & 4.3032 & 5.5603 \\
\hline
\end{tabular}

Table 8 illustrates the effect of message size on the maximum throughput measured when $N=24$. It is noticed that the results follow the same pattern as in the no-cloning case study. The throughput deteriorates with an increase in message size for any given architecture. This is because more work needs to be performed per client cycle with larger messages. Moreover, by comparing the maximum throughputs obtained in both the nocloning and the 4-degree cloned architectures it is clear that the agent cloning has caused a substantial improvement in the overall system throughput by stretching the bottleneck. The throughput increase of $76 \%$ and $216 \%$ in the F-ORB and the P-ORB respectively manifests this fact.

\subsubsection{Eight-Degree Cloned Architectures:}

Following the same logic described in Subsection 5.2.4.2, the H-ORB maintains its nocloning status and the issue of cloning is confined to the F-ORB and P-ORB architectures. Hence, there will be eight $f$-agents and eight p-agents in each of the respective architectures in addition to the default agent supplied by ORBeline. The clone processes are forked in the same manner as in the previous case study. Increasing the degree of cloning from (4) 


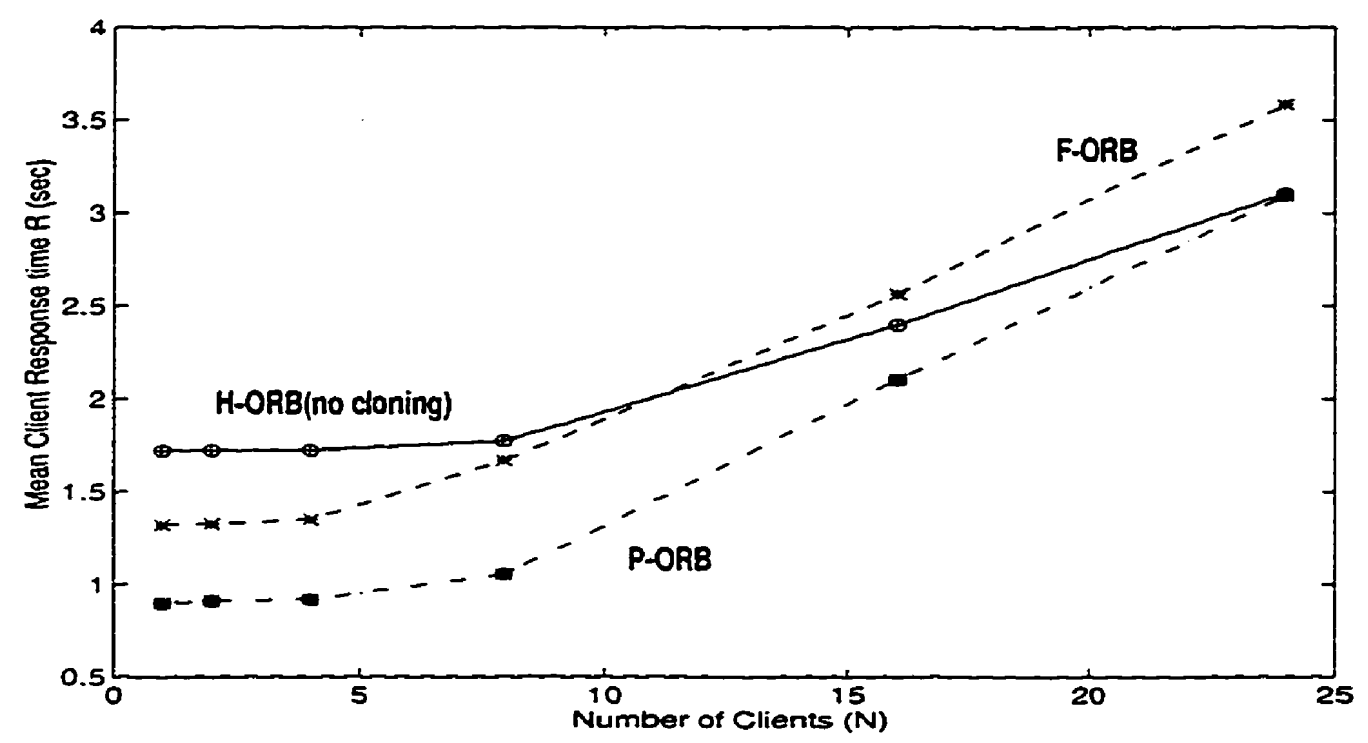

Figure 5-18: The Response Time of the H-ORB, the F-ORB and the P$O R B$ with $L=4800$ Bytes, and 8 Cloning Degrees.

Degree of Cloning $=8, D=200 \mathrm{~ms}, \mathrm{~L}=4800$ Bytes, $\mathrm{S}_{A}=10 \mathrm{~ms}, \mathrm{~S}_{B}=15 \mathrm{~ms}$

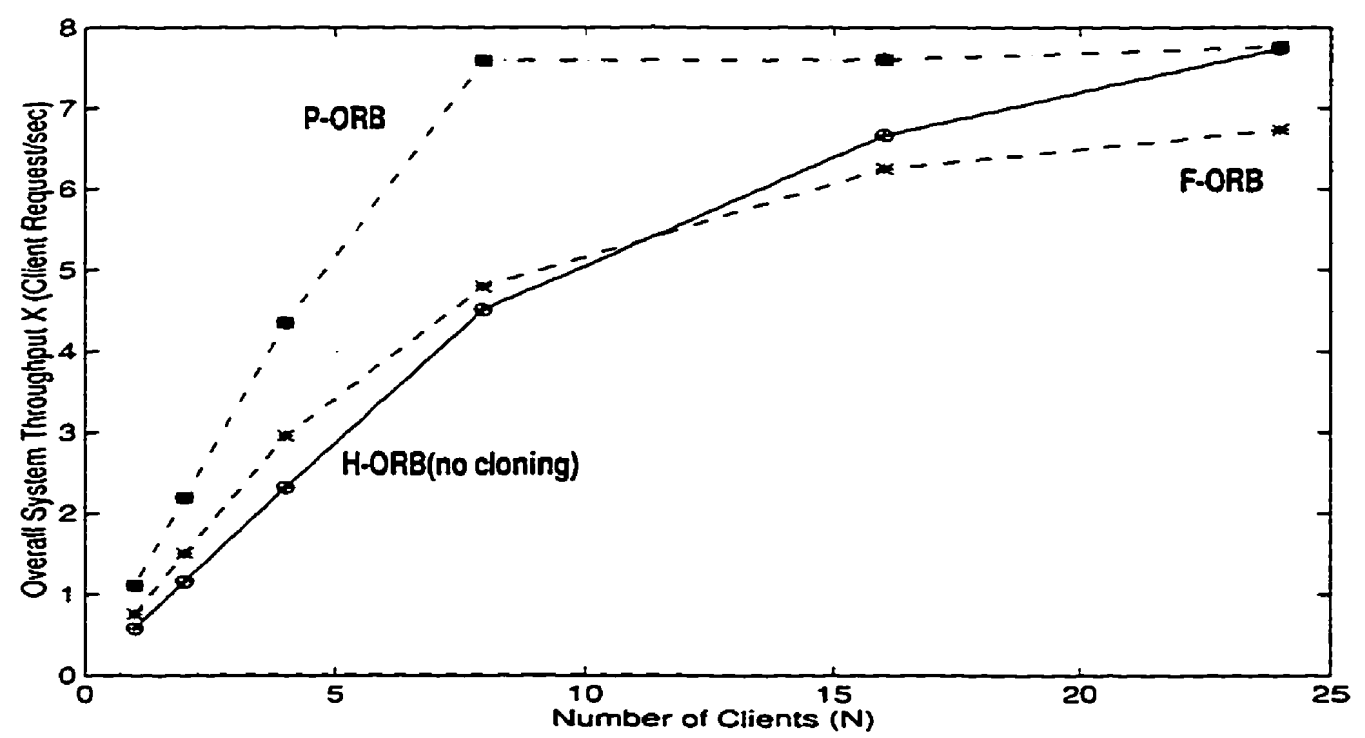

Figure 5-19: The Throughput of the H-ORB, the F-ORB and the P-ORB with $\mathrm{L}=4800$ Bytes, and 8 Cloning Degrees.

Degree of Cloning $=8, D=200 \mathrm{~ms}, L=4800$ Bytes, $S_{A}=10 \mathrm{~ms}, S_{B}=15 \mathrm{~ms}$ 


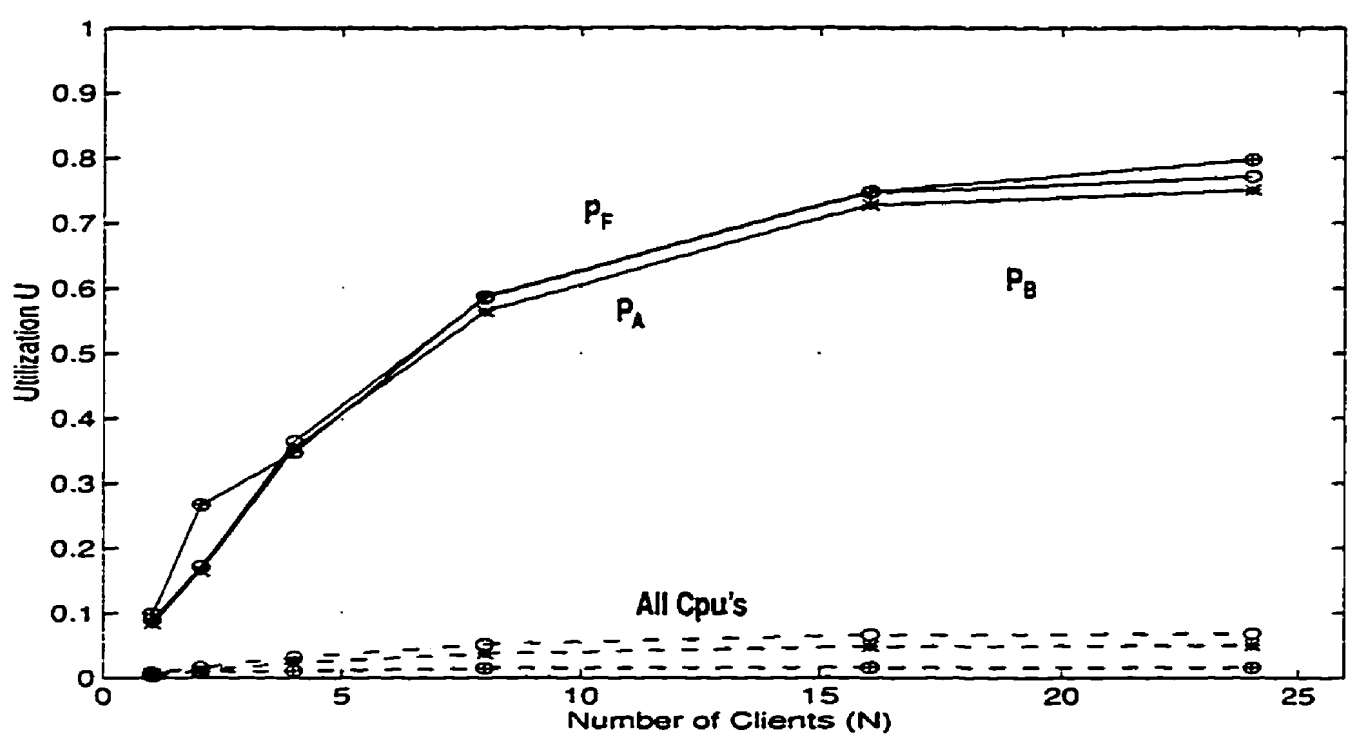

Figure 5-20: Cpu \&Process Utilizations of the F-ORB with $L=4800$ Bytes and 8 Cloning Degrees.

$P_{A}=$ Process Util. of Server $A, C P U_{A}=C p u$ Util of Server A.P $P_{F}=$ Process Util. of f-agent. Degree of Cloning $=8, D=200 \mathrm{~ms}, L=4800$ Bytes, $S_{A}=10 \mathrm{~ms}, S_{B}=15 \mathrm{~ms}$

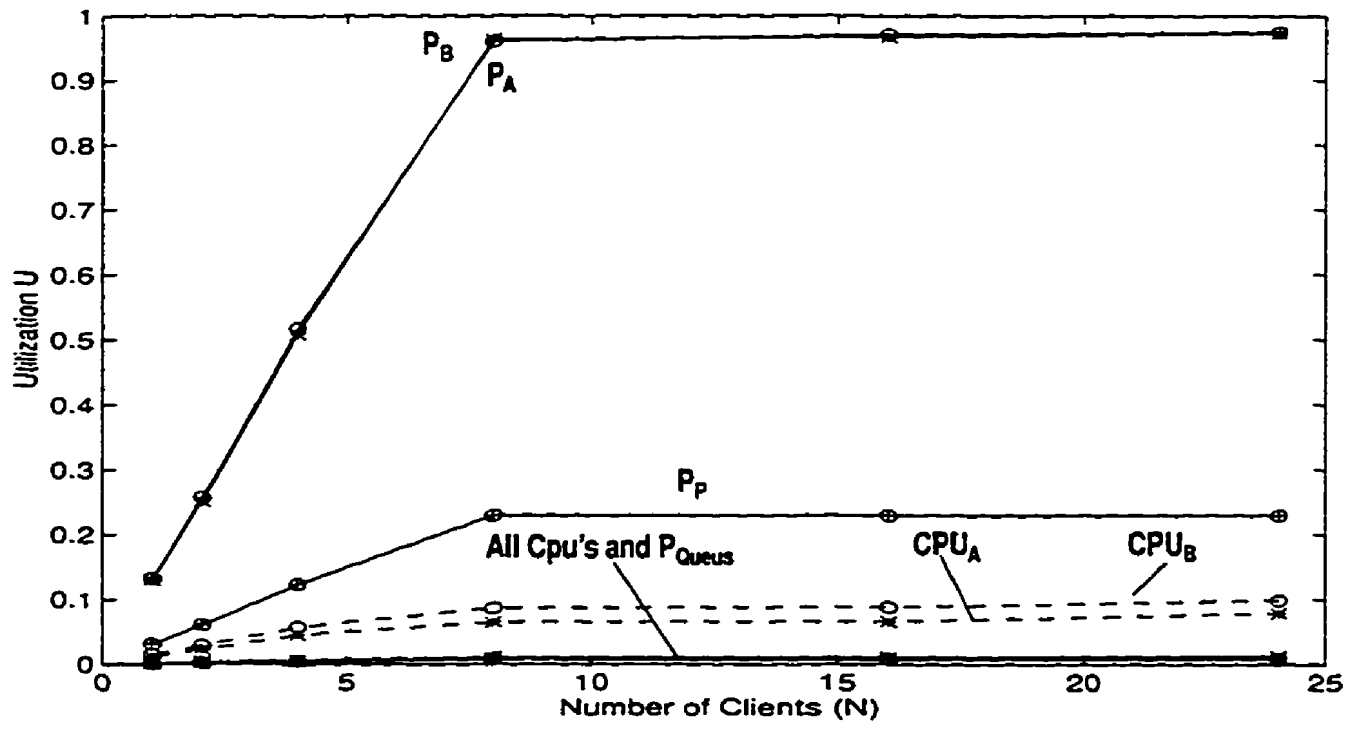

Figure 5-21: Cpu \&Process Utilizations of the $\mathrm{P}-\mathrm{ORB}$ with $\mathrm{L}=\mathbf{4 8 0 0}$ Bytes and 8 Cloning Degrees.

$\mathrm{P}_{\mathrm{A}}=$ Process Util. of Server $A, C P U_{A}=C p u$ Util of Server $A . P_{P}=$ Process Util. of p-agent. $P_{\text {Queues }}=$ Process Util. of dispatcher, Degree of Cloning $=8, D=200 \mathrm{~ms}, L=4800$ Bytes, $\mathrm{S}_{\mathrm{A}}=10 \mathrm{~ms}, \mathrm{~S}_{\mathrm{B}}=15 \mathrm{~ms}$ 
to (8) caused a further reduction of the queueing delays that the requests encounter at the agent processes as noted in this set of experimental results.

Message Size - 4800 Bytes: The mean client response time and the corresponding system throughput of the three architectures are captured in Figure 5-18 and Figure 5-19 respectively, and the related utilization graphs are in Figure 5-20 and Figure 5-21. As can be observed from the figures and Table 9, the cloning has significantly improved the overall system throughput for the F-ORB and the P-ORB architectures by $135 \%$ and $319 \%$, respectively, due to the load distribution on the eight agents and the consequent reduction of the queueing time at the agents for all incoming requests/replies. Also, as a result of this improvement in performance, the P-ORB maintains its supremacy over the whole range of $\mathrm{N}$ and the crossover point of the H-ORB with the F-ORB architectures has been shifted to a higher value of $\mathrm{N}$ than that of the 4-degree cloning.

Table 9: Effect of $L$ on Latency at $N=1\left(D=200 \mathrm{~ms}, S_{A} / S_{B}=10 \mathrm{~ms} / 15 \mathrm{~ms}, 8\right.$ Cloning Degrees)

\begin{tabular}{|c|c|c|c|}
\hline L (bytes) & $\begin{array}{c}\text { H-ORB (sec) } \\
\text { (no Cloning) }\end{array}$ & F-ORB (sec) & P-ORB (sec) \\
\hline 4800 & 1.7212 & 1.3210 & 0.8976 \\
\hline 9600 & 1.7425 & 1.3529 & 0.9053 \\
\hline 19200 & 1.7933 & 1.4366 & 0.9150 \\
\hline
\end{tabular}

Message Size - 9600 Bytes: Similar behavioral patterns were observed as shown in Figure A-22 to Figure A-25 in Appendix A. 
Table 10: Throughput at $N=24\left(D=200 \mathrm{~ms}, S_{A} / S_{B}=10 \mathrm{~ms} / 15 \mathrm{~ms}, 8\right.$ Cloning Degrees)

\begin{tabular}{|c|c|c|c|}
\hline $\begin{array}{c}\mathrm{L} \\
\text { (Byres) }\end{array}$ & $\begin{array}{c}\text { H-ORB } \\
\text { (Client Request/sec) }\end{array}$ & $\begin{array}{c}\text { F-ORB V1 } \\
\text { (Client Request/sec) }\end{array}$ & $\begin{array}{c}\text { P-ORB } \\
\text { (Client Reques/sec) }\end{array}$ \\
\hline 4800 & 7.7356 & 6.7353 & 7.7618 \\
\hline 9600 & 7.4533 & 6.4870 & 7.6175 \\
\hline 19200 & 6.8688 & 5.7304 & 7.4971 \\
\hline
\end{tabular}

Message Size - 19200 Bytes: Similar behavioral patterns to the previous case studies of were also observed as shown in Figure A-26 to Figure A-29 in Appendix A.

The effect of message size on the maximum throughput observed at $\mathrm{N}=24$ is displayed in Table 10 for the three different architectures and it is clear that it is following the same pattern as in the two previous cloning degrees case studies.

\subsubsection{The Impact of the Server Demand}

Client requests can be characterized according to their computational demands at the servers nodes. This set of experiments is designed to investigate the relative performance of the three main architectures as the computational demand for each request per server class increases over a range of cloning degrees (a variable number of f-agents and pagents). The experiments main parameters are listed in Table 11 with the variable parameters in italic fonts. The set of graphs shown in this section represents the results obtained for a representative set of the server demands for both classes of servers. 
Table 11: Parameters of Server Demand Experiments

\begin{tabular}{|l|l|}
\hline \multicolumn{1}{|c|}{ Main Parameters } & \multicolumn{1}{|c|}{ Range of values } \\
\hline Number of Clients & $1,2,4,816,24$ \\
\hline Service Times & $\begin{array}{l}\text { Class } A \text { server }=10 \mathrm{~ms}, 50 \mathrm{~ms}, 250 \mathrm{~ms} \\
\text { Class } B \text { server }=15 \mathrm{~ms}, 75 \mathrm{~ms}, 375 \mathrm{~ms}\end{array}$ \\
\hline Inter-Node Delay & $10 \mathrm{~ms}$ \\
\hline Message size & 150 Bytes \\
\hline $\begin{array}{l}\text { Degree of Cloning: } \\
\begin{array}{l}\text { H-ORB Arch. } \\
\text { P-ORB Arch } \text { Arch. }\end{array}\end{array}$ & $\begin{array}{l}\text { Default Agent }=1 \\
\text { Default Agent }=1, \text { F-agents }=1,4,8 \\
\text { Default Agent }=1, P \text {-agents }=1,4,8\end{array}$ \\
\hline
\end{tabular}

The remaining results can be consulted in Appendix B Figure B-1 to Figure B-25. More experiments need to be done to compare the performance of both of the F-ORB.VI and FORB.V2 architectures. Hence all experiment results in this set correspond to the performance of the F-ORB.Vl architecture.

\subsubsection{No Cloning (Single Agent) Architectures:}

Class A: $10 \mathrm{~ms}$, Class B: $15 \mathrm{~ms}$ - The influence of the cpu demand as the number of clients increases on the mean client response time and the corresponding overall mean system throughput is investigated at different level of system loading.

\section{Low Load Conditions:}

As seen in Table 12, Figure 5-22 and Figure 5-23, when $\mathrm{N}=1$, all architectures exhibit an increase in the latency with the increase in the computational demands of the servers. Since the inter-node delay $\mathrm{D}$ and the message size $\mathrm{L}$ are set to be small, the primary factor affecting the mean client response time is the servers demands. For any given combination 
of $\mathrm{S}_{\mathrm{A}}$ and $\mathrm{S}_{\mathrm{B}}$, the $\mathrm{P}-\mathrm{ORB}$ produces the smallest response time due to the concurrent invocation of the servers.

Table 12: Effect of $S_{A} / S_{B}$ on Latency at $N=1(D=10 \mathrm{~ms}, L=150$ Bytes, no Cloning)

\begin{tabular}{|c|c|c|c|}
\hline $\mathbf{S}_{\mathbf{A}}, \mathbf{S}_{\mathbf{B}}$ (ms) & H-ORB (sec) & F-ORB (sec) & P-ORB (sec) \\
\hline 10,15 & 0.1786 & 0.1838 & 0.1733 \\
\hline 50,75 & 0.2844 & 0.2502 & 0.1979 \\
\hline 250,375 & 0.8238 & 0.9518 & 0.5209 \\
\hline
\end{tabular}

\section{Medium to High Load Conditions:}

As $\mathrm{N}$ goes beyond 2, the H-ORB starts to take the lead and provides a high throughput until $N$ reaches 16 at which time this architecture can not give more throughput due to the saturation of both servers as shown in Figure 5-24. As discussed earlier in Subsection 5.2.4, the scalability of the F-ORB and the P-ORB is severely limited due to the presence of software bottlenecks, at the f-agent and the p-agent respectively. The analysis of the process utilizations of the aforementioned processes in Figure 5-25 and Figure 5-26 respectively proves this fact.

Class A: $50 \mathrm{~ms}$, Class B: $75 \mathrm{~ms}$ - The mean client response time the related overall mean system throughput are shown in Appendix B in Figure B-1 and Figure B-2, and the corresponding utilization graphs are in Figure B-3 to Figure B-5. Analyzing those figures reveals that the system performance follows a relatively similar pattern to that of the previous case study. One observation worth mentioning is that when the system is lightly loaded ( $\mathrm{N}$ is fewer than 4 ), the P-ORB behaves best due to the effect of the concurrent 


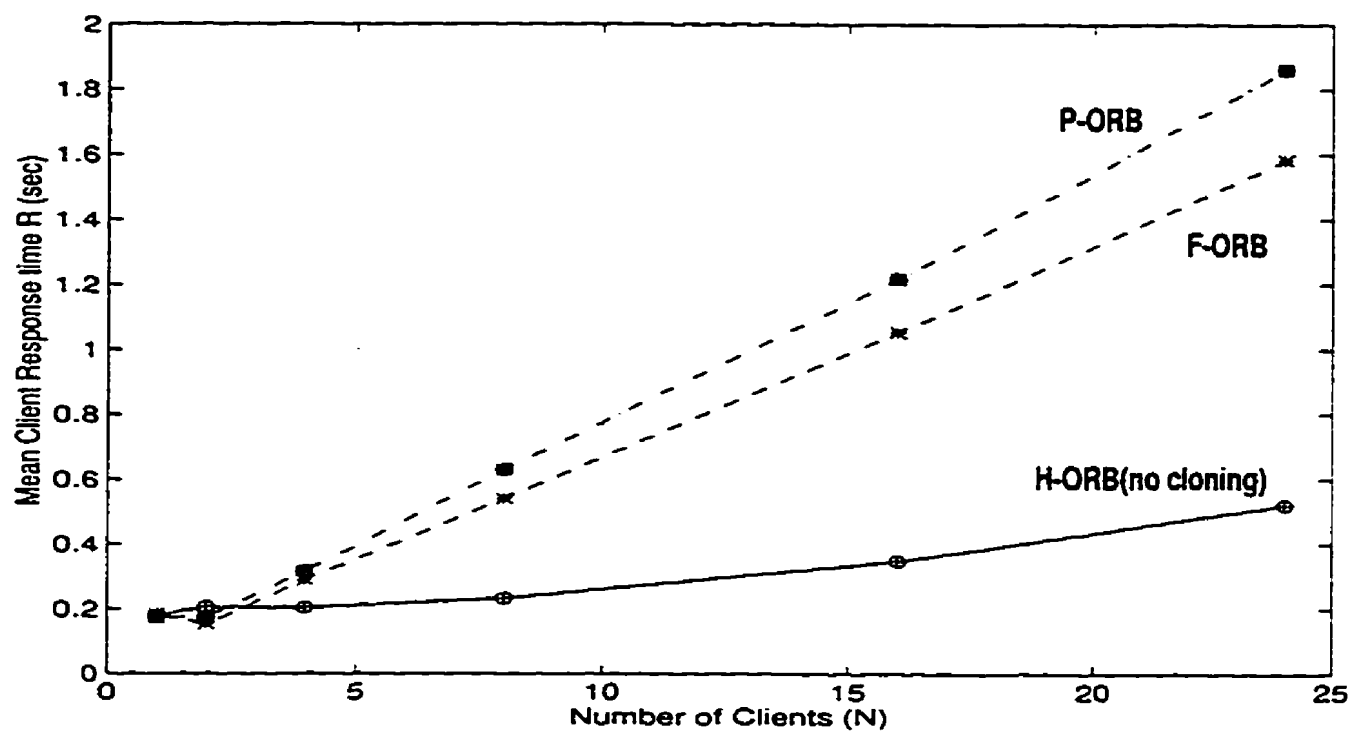

Figure 5-22: The Response Time of the H-ORB, the F-ORB and the PORB with $S_{A} / S_{B}=10 / 15 m s$, and no Cloning.

Degree of Cloning $=1, D=10 \mathrm{~ms}, L=150$ Bytes, $S_{A}=10 \mathrm{~ms}, S_{B}=15 \mathrm{~ms}$

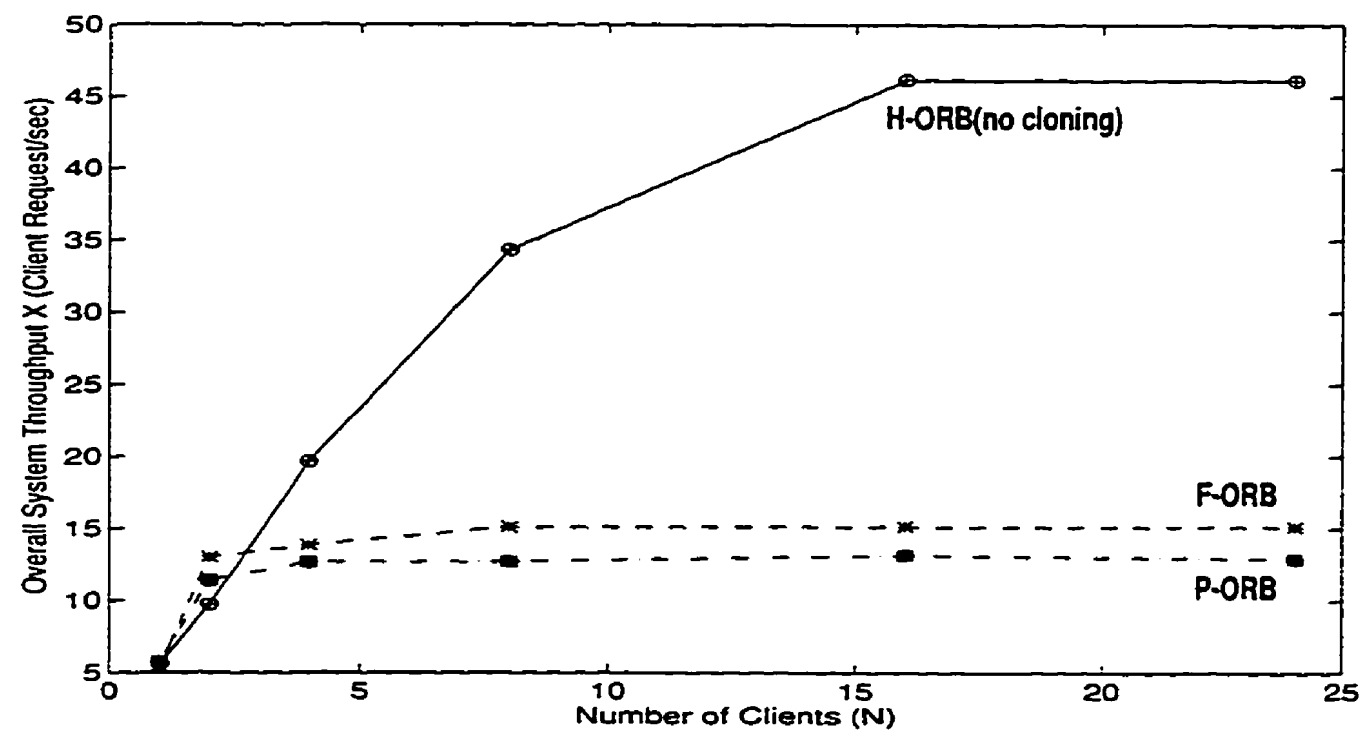

Figure 5-23: The Throughput of the H-ORB, the F-ORB and the P-ORB with $S_{A} / S_{B}=10 / 15 m s$, and no Cloning.

Degree of Cloning $=1, D=10 \mathrm{~ms}, L=150$ Bytes, $S_{A}=10 \mathrm{~ms}, S_{B}=15 \mathrm{~ms}$ 


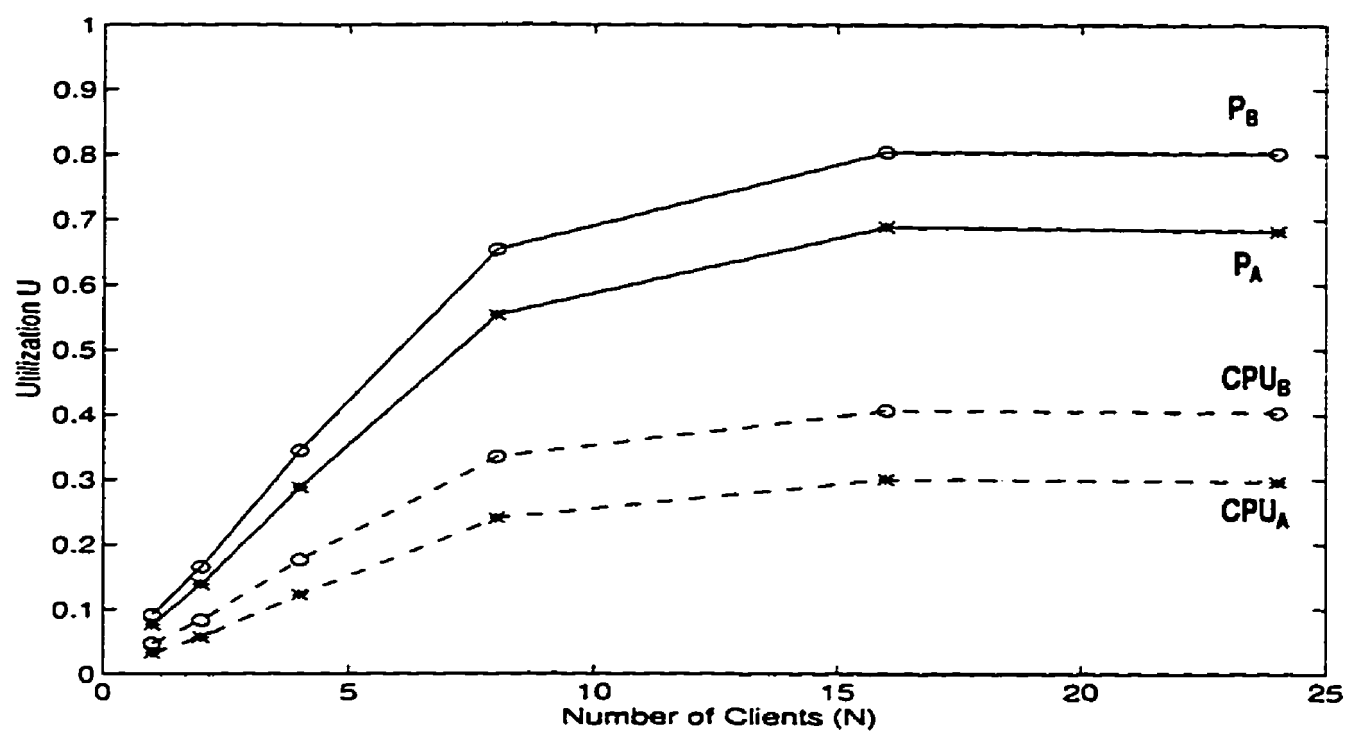

Figure 5-24: Cpu and Process Utilizations of the H-ORB with $\mathrm{S}_{\mathrm{A}} / \mathrm{S}_{\mathrm{B}}=10 / 15 \mathrm{~ms}$, and no Cloning.

$P_{A}=$ Process Util. of Server $A, C P U_{A}=C p u$ Util of Server A.

Degree of Cloning $=1, D=10 \mathrm{~ms}, \mathrm{~L}=150$ Bytes, $S_{A}=10 \mathrm{~ms}, S_{B}=15 \mathrm{~ms}$

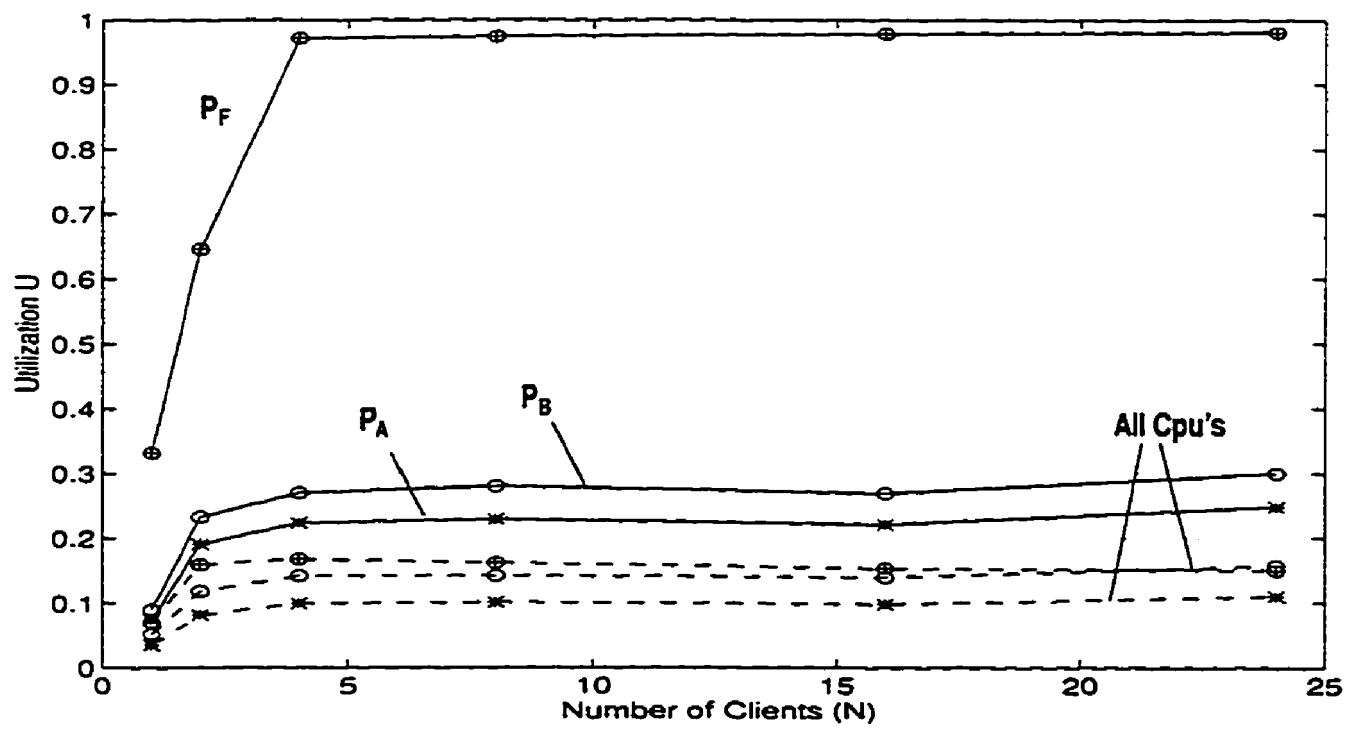

Figure 5-25: Cpu \&Process Utilizations of the F-ORB with $S_{A} / S_{B}=10 / 15 m s$, and no Cloning.

$P_{A}=$ Process Util. of Server $A, C P U_{A}=C p u$ Util of Server $A, P_{F}=$ Process Util. of f-agent. Degree of Cloning $=1, D=10 \mathrm{~ms}, \mathrm{~L}=150$ Bytes, $S_{A}=10 \mathrm{~ms}, S_{B}=15 \mathrm{~ms}$ 


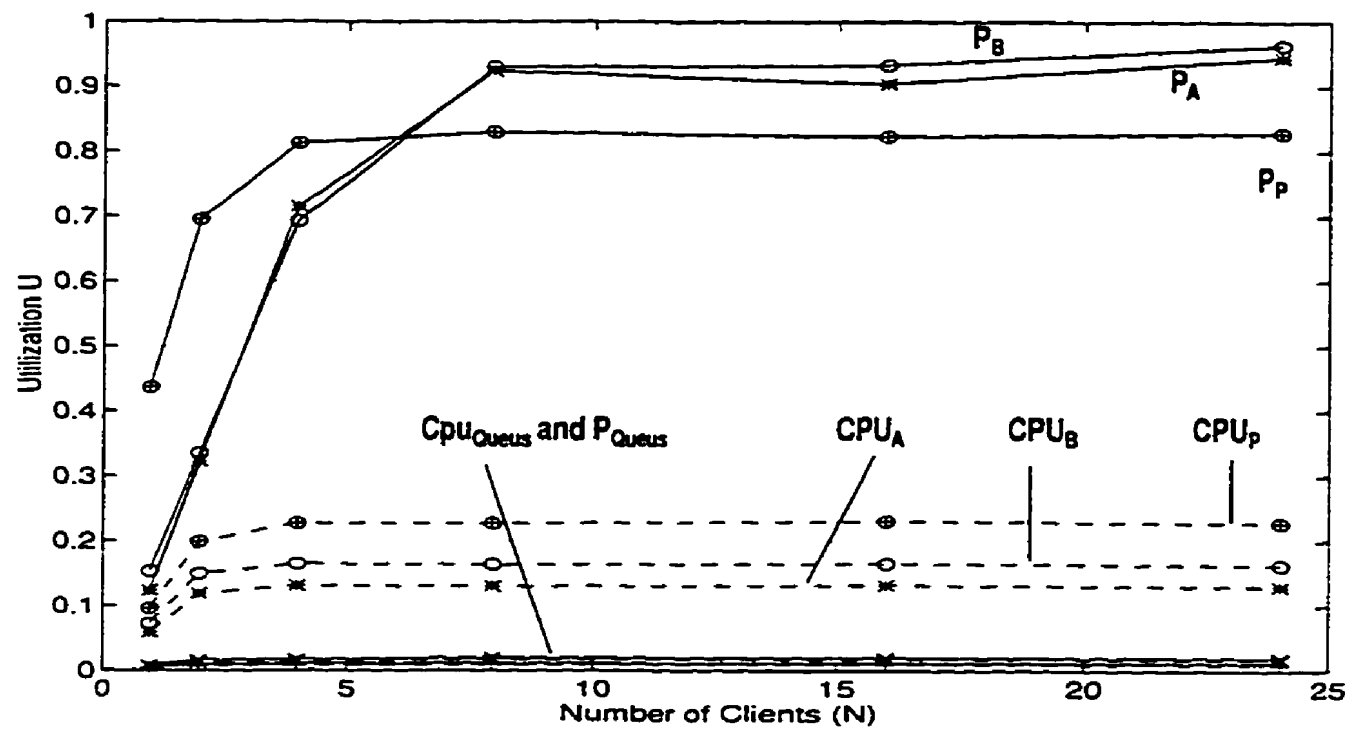

Figure 5-26: Cpu \&Process Utilizations of the P-ORB with $S_{A} / S_{B}=10 / 15 m s$, and no Cloning.

$P_{A}=$ Process Util. of Server $A, C P U_{A}=C p u$ Util of Server $A, P_{P}=$ Process Util. of p-agent.

$P_{\text {Queues }}=$ Process Util. of dispatcher, Degree of Cloning $=1, D=10 \mathrm{~ms}, L=150$ Byres,

$\mathrm{S}_{\mathrm{A}}=10 \mathrm{~ms}, \mathrm{~S}_{\mathrm{B}}=15 \mathrm{~ms}$

invocation of the servers. Since the server demands are higher, the lead of the P-ORB is clear.

Class A: $250 \mathrm{~ms}$, Class B: $375 \mathrm{~ms}$ - The mean client response time and the overall mean system throughput under low to high system loading are investigated.

\section{Low Load Conditions:}

As shown in Table 12 and in Figure 5-27 and Figure 5-28, when $\mathrm{N}=1$, the $\mathrm{P}-\mathrm{ORB}$ has the best latency since there is no queueing delays and the client's request cycle time is mainly affected by the concurrent invocation of the servers. The H-ORB has a slight lead over the F-ORB since the message interchanged between the client and the default agent is smaller in addition to the fact that the agent has less work to do compared to the f-agent. 
Hence the default agent is faster than the f-agent

\section{Medium to High Load Conditions:}

As shown in Table 13 and in Figure 5-28, both the H-ORB and the P-ORB throughputs start to flatten as $\mathrm{N}$ increases due to the saturation of server class $\mathrm{B}$ and give very close results at $\mathrm{N}=24$. However, the $\mathrm{F}-\mathrm{ORB}$ behaves badly due to the early saturation of the $\mathrm{f}$ agent and the consequent increase in the queueing time at the agent. Figure 5-29 to Figure 5-31 can be consulted for more information on the various utilization levels for the three architectures.

Table 13: Throughput at $N=24(D=10 \mathrm{~ms}, L=150$ Bytes, no Cloning)

\begin{tabular}{|c|c|c|c|}
\hline $\begin{array}{c}\mathbf{S}_{\mathbf{A}}, \mathbf{S}_{\mathbf{B}} \\
(\mathrm{ms})\end{array}$ & $\begin{array}{c}\text { H-ORB } \\
\text { (Client Request/sec) }\end{array}$ & $\begin{array}{c}\text { F-ORB } \\
\text { (Client Reques/sec) }\end{array}$ & $\begin{array}{c}\text { P-ORB } \\
\text { (Client Request/sec) }\end{array}$ \\
\hline 10,15 & 46.1460 & 15.1543 & 12.9206 \\
\hline 50,75 & 16.1142 & 11.8707 & 11.4470 \\
\hline 250,375 & 4.0213 & 2.7032 & 4.1040 \\
\hline
\end{tabular}

\subsubsection{Four-Degree Cloned Architectures:}

The cloning approach is used again to alleviate the software bottlenecks encountered in the no-cloning case study. As explained in Subsection 5.2.4.2, the H-ORB retains its nocloning status while each of the F-ORB and P-ORB will have four $f$-agents and four pagents respectively, in addition to the default agent

Class A: $10 \mathrm{~ms}$, Class B: $15 \mathrm{~ms}$ - Figure 5-32 and Figure 5-33 show the mean client response time and the corresponding overall mean system throughput of the three 


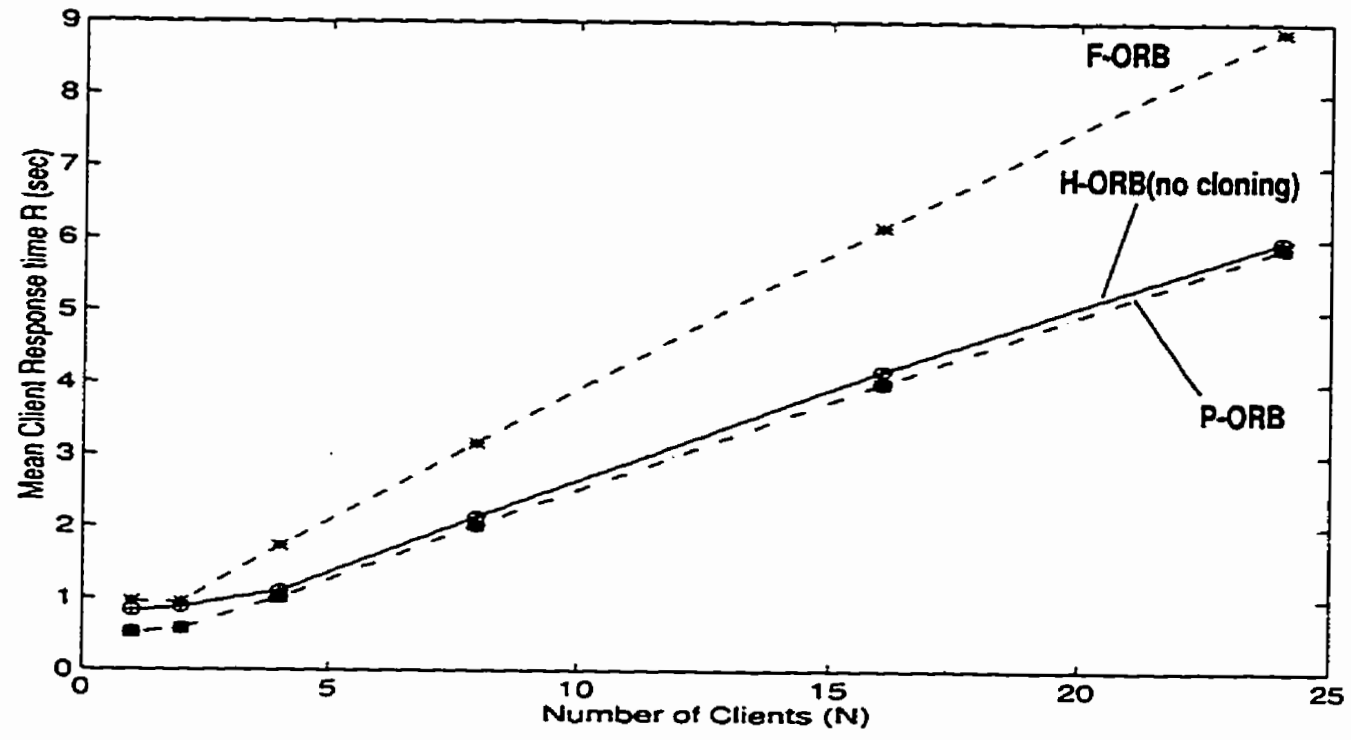

Figure 5-27: The Response Time of the H-ORB, the F-ORB and the P$O R B$ with $S_{A} / S_{B}=250 / 375 m s$, and no Cloning.

Degree of Cloning $=1, D=10 \mathrm{~ms}, L=150$ Bytes, $S_{A}=250 \mathrm{~ms}, S_{B}=375 \mathrm{~ms}$

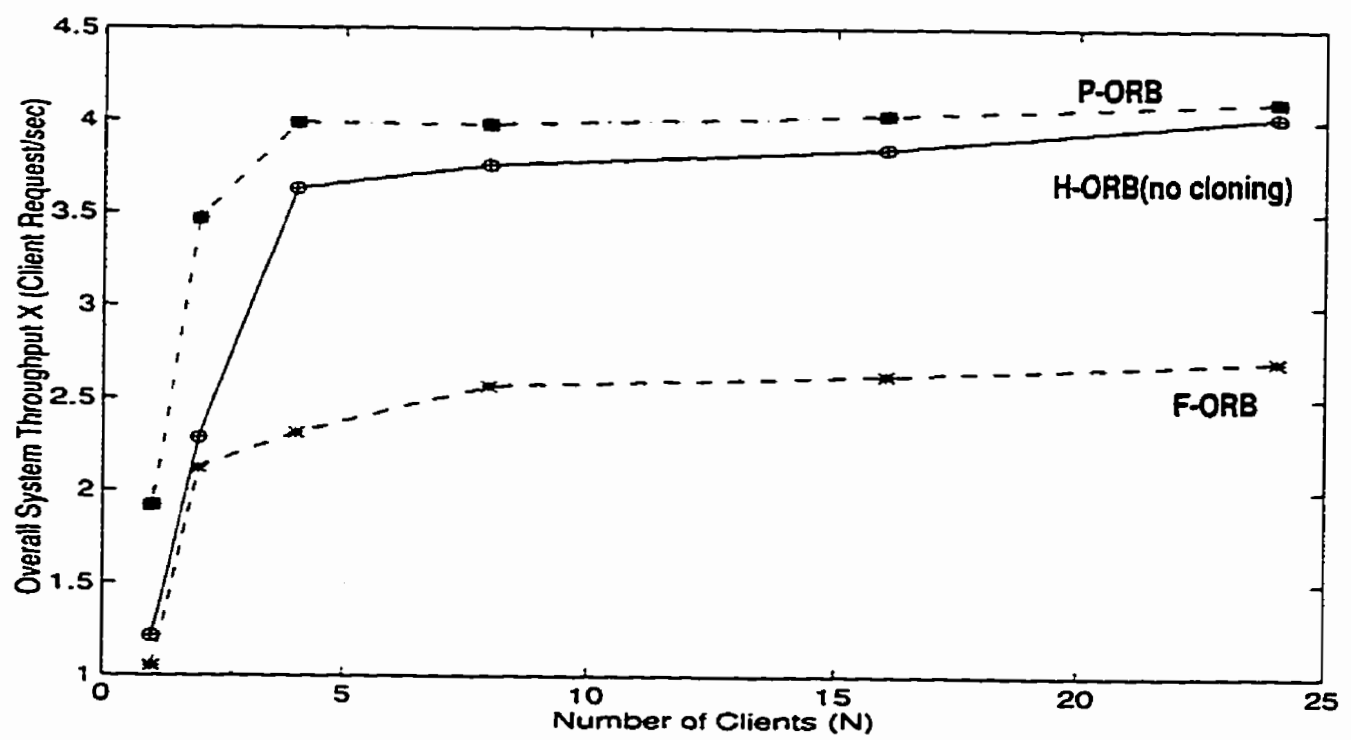

Figure 5-28: The Throughput of the H-ORB, the F-ORB and the P-ORB with $S_{A} / S_{B}=250 / 375 m s$, and no Cloning.

Degree of Cloning $=1, D=10 \mathrm{~ms}, L=150$ Bytes, $S_{A}=250 \mathrm{~ms}, S_{B}=375 \mathrm{~ms}$ 


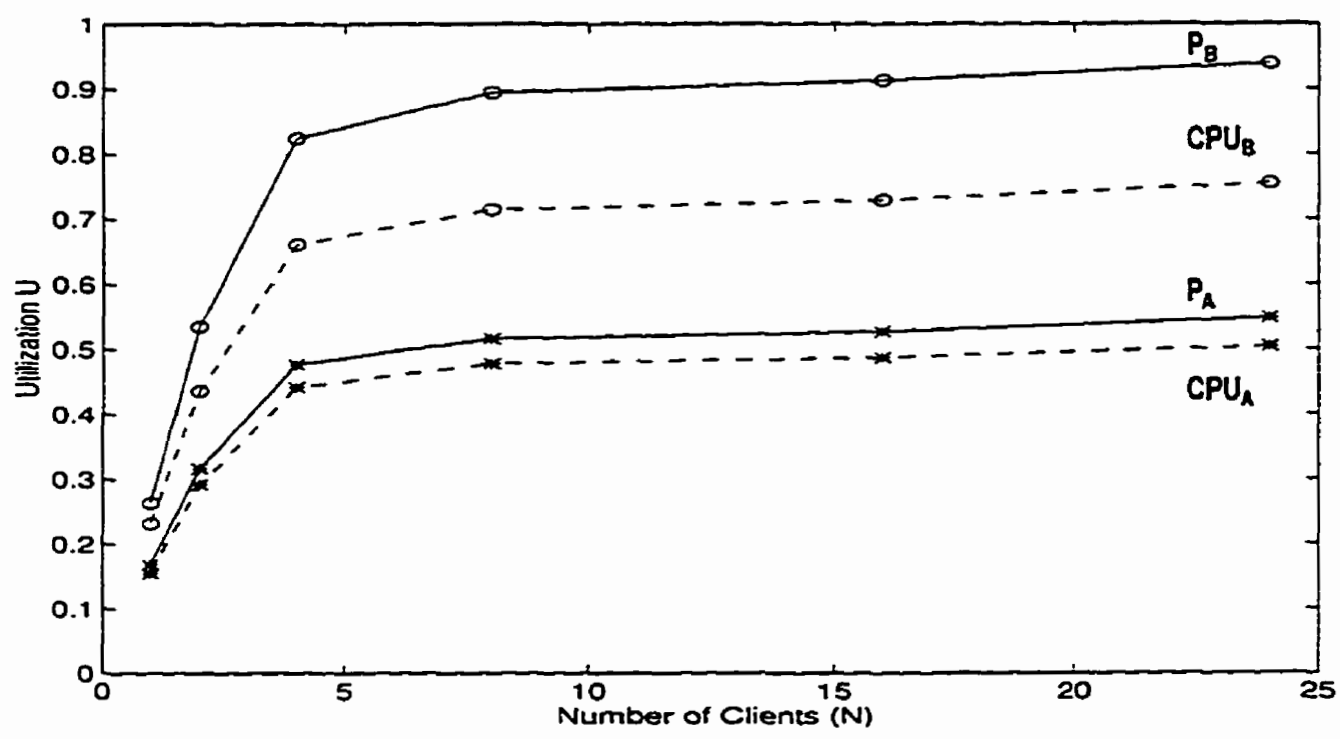

Figure 5-29: Cpu and Process Utilizations of the H-ORB with $\mathrm{S}_{\mathrm{A}} / \mathrm{S}_{\mathrm{B}}=250 / 375 \mathrm{~ms}$, and no Cloning.

$P_{A}=$ Process Util. of Server $A, C P U_{A}=C$ pu Util of Server $A$.

Degree of Cloning $=1, D=10 \mathrm{~ms}, L=150$ Bytes, $S_{A}=250 \mathrm{~ms}, S_{B}=375 \mathrm{~ms}$

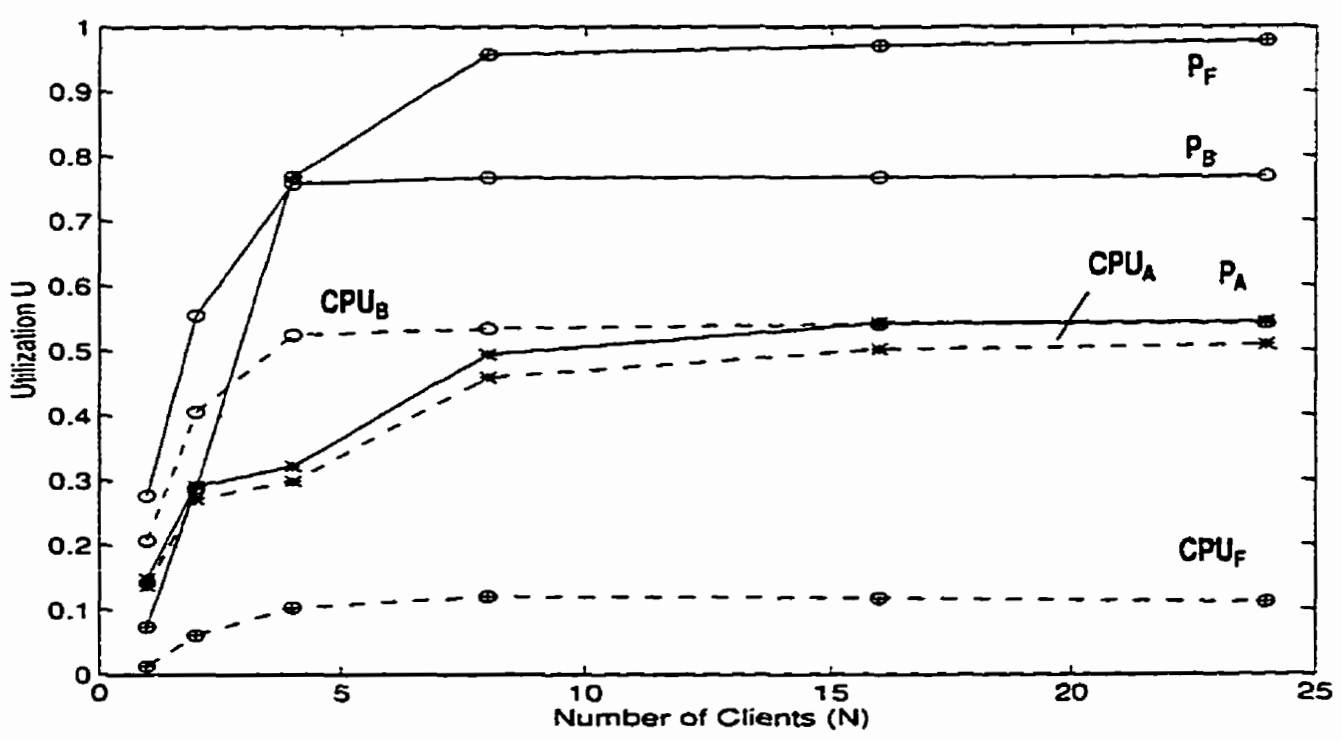

Figure 5-30: Cpu \&Process Utilizations of the F-ORB with $S_{A} / S_{B}=250 / 375 m s$, and no Cloning.

$P_{A}=$ Process Util. of Server $A, C P U_{A}=C$ pu Util of Server $A, P_{F}=$ Process Util. of $f$-agent.

Degree of Cloning $=1, D=10 \mathrm{~ms}, \mathrm{~L}=150$ Bytes, $S_{A}=250 \mathrm{~ms}, S_{B}=375 \mathrm{~ms}$ 


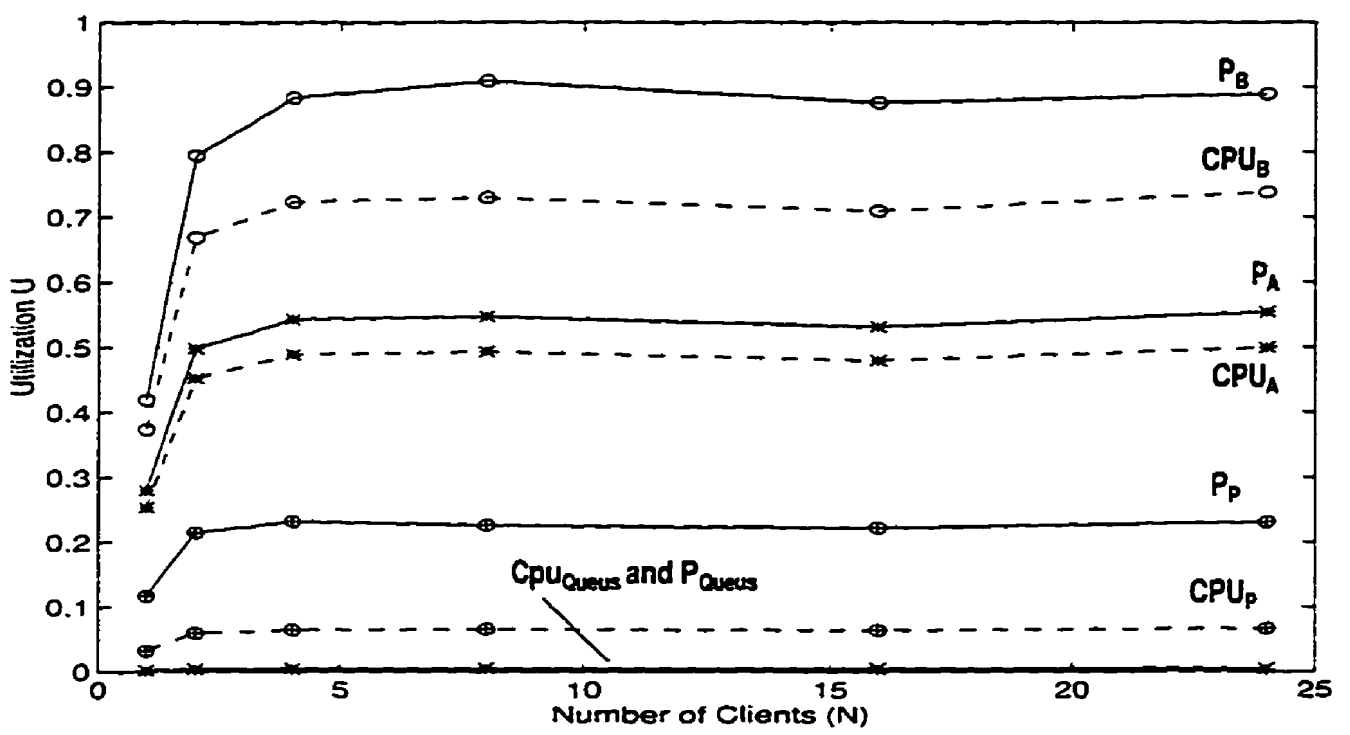

Figure 5-31: Cpu \&Process Utilizations of the P-ORB with $S_{A} / S_{B}=250 / 375 m s$, and no Cloning.

$P_{A}=$ Process Util. of Server $A, C P U_{A}=C$ pu Util of Server $A, P_{p}=P$ rocess Util. of p-agent.

$P_{\text {Queves }}=$ Process Util. of dispatcher, Degree of Cloning $=I, D=10 \mathrm{~ms}, L=150$ Bytes,

$\mathrm{S}_{\mathrm{A}}=250 \mathrm{~ms}, \mathrm{~S}_{\mathrm{B}}=375 \mathrm{~ms}$

architectures with 4 degrees of cloning. As can be observed from the figures and Table 14, the cloning has significantly improved the overall system throughput for the F-ORB and the $\mathrm{P}-\mathrm{ORB}$ architectures due to the load distribution on the four agents and the consequent reduction of the queueing time at the agents for all incoming requests/replies. Also, as a result of this improvement in performance, the crossover point of the H-ORB with the other two architectures has been shifted to higher values of $\mathrm{N}$ than that of the no-cloning case.

Table 14: Effect of $S_{A} / S_{B}$ on Latency at $N=1(D=10 \mathrm{~ms}, L=150$ Bytes, 4 Cloning Degrees)

\begin{tabular}{|c|c|c|c|}
\hline $\mathbf{S}_{\mathbf{A}}, \mathbf{S}_{\mathbf{B}}$ (ms) & $\begin{array}{c}\text { H-ORB (sec) } \\
\text { (no cloning) }\end{array}$ & F-ORB (sec) & P-ORB (sec) \\
\hline 10,15 & 0.1786 & 0.1521 & 0.1362 \\
\hline 50,75 & 0.2844 & 0.2601 & 0.1969 \\
\hline 250,375 & 0.8238 & 0.8436 & 0.5163 \\
\hline
\end{tabular}


However, the P-ORB behaved worse than the F-ORB.V1 due to the early saturation of the class B server.

Table 15: Throughput at $N=24(D=10 \mathrm{~ms}, \mathrm{~L}=150$ Bytes, 4 Cloning Degrees)

\begin{tabular}{|c|c|c|c|}
\hline $\begin{array}{c}\mathbf{S}_{\mathbf{A}}, \mathbf{S}_{\mathbf{B}} \\
(\mathrm{ms})\end{array}$ & $\begin{array}{c}\mathbf{H}-\mathbf{O R B} \\
\text { (no cloning) } \\
\text { (Client Request/sec) }\end{array}$ & $\begin{array}{c}\text { F-ORB } \\
\text { (Client Reques/sec) }\end{array}$ & $\begin{array}{c}\text { P-ORB } \\
\text { (Client Request/sec) }\end{array}$ \\
\hline 10,15 & 46.1460 & 30.2729 & 20.1084 \\
\hline 50,75 & 16.1142 & 12.3782 & 13.8401 \\
\hline 250,375 & 4.0213 & 2.5871 & 4.3269 \\
\hline
\end{tabular}

Class A: $50 \mathrm{~ms}$, Class B: $75 \mathrm{~ms}$ - The mean client response time and the corresponding overall system throughput are shown on Figure B-6 and Figure B-7 in Appendix B.With four degrees of cloning, both the F-ORB and the P-ORB architectures have produced higher throughputs. At low number of clients $\mathrm{N}$, identical behavioral patterns to the respective no-cloning case were obtained from all architectures. At higher values of $\mathrm{N}$, the P-ORB surpassed the F-ORB. The utilization graphs are also presented in Appendix B in Figure B-8 and Figure B-9.

Class A: $250 \mathrm{~ms}$, Class B: $375 \mathrm{~ms}$ - Similar behavioral patterns to the corresponding nocloning case were observed. Figure B-10 to Figure B-13 in Appendix B give the mean client response time, the respective overall system throughput of all three architectures and their utilization graphs. Cloning of the agent does not seem to affect the system performance since the large service times of both classes of servers seem to dominate system performance. 


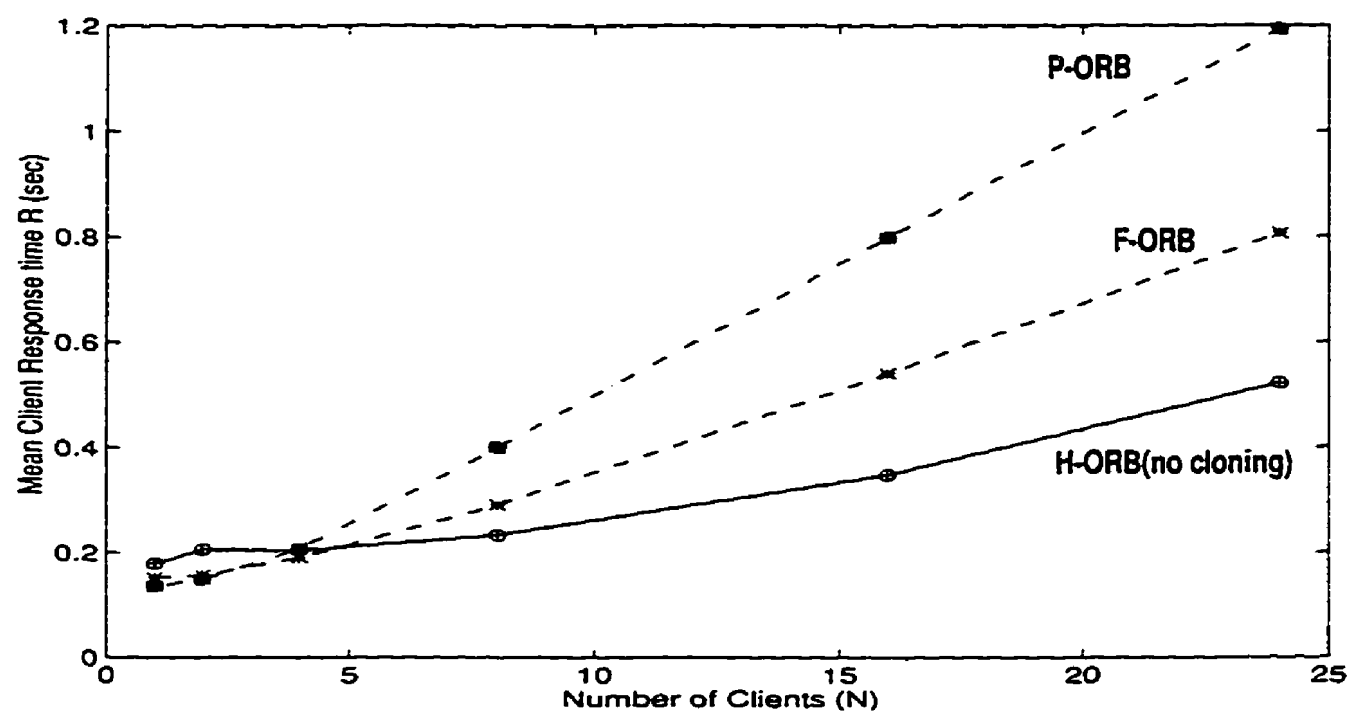

Figure 5-32: The Response Time of the H-ORB, the F-ORB and the $P$ ORB with $S_{A} / S_{B}=10 / 15 m s$, and 4 Cloning Degrees.

Degree of Cloning $=4, D=10 \mathrm{~ms}, L=150$ Bytes, $S_{A}=10 \mathrm{~ms}, S_{B}=15 \mathrm{~ms}$

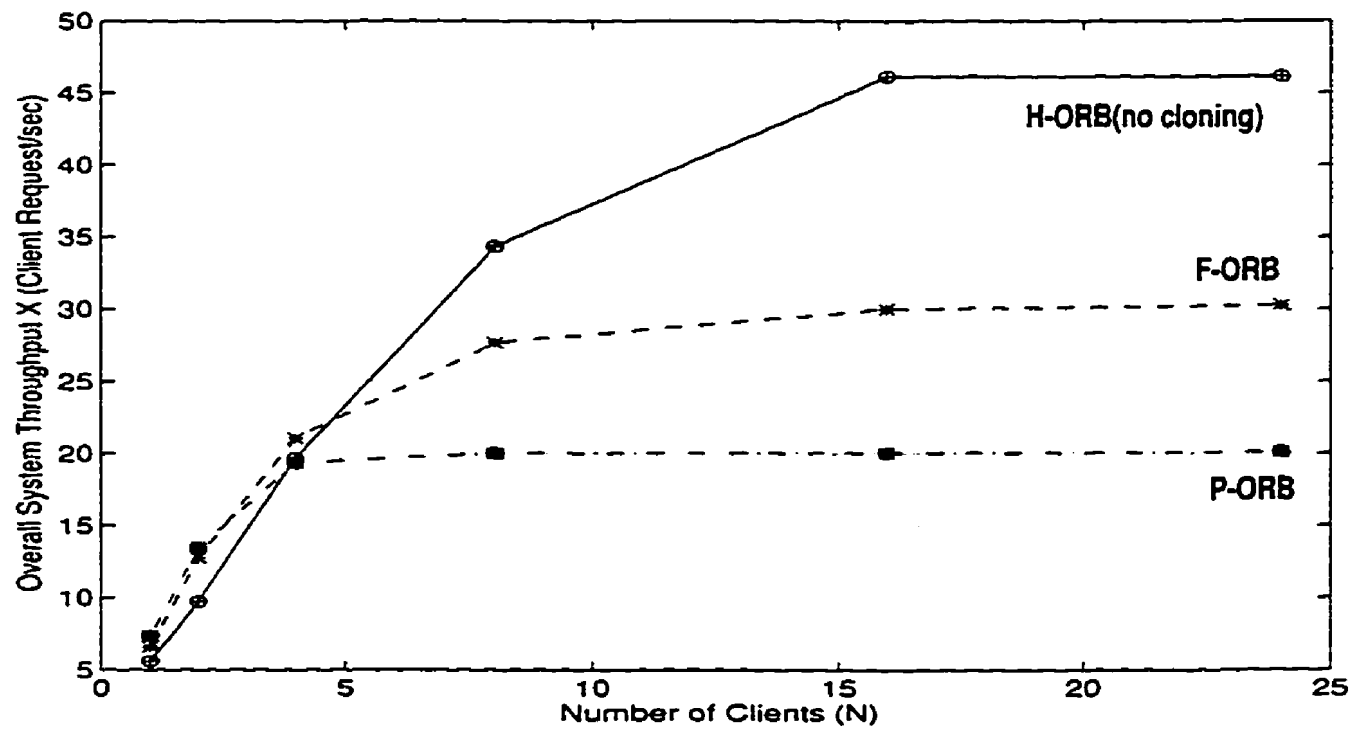

Figure 5-33: The Throughput of the H-ORB, the F-ORB and the P-ORB with $S_{A} / S_{B}=10 / 15 m s, 4$ Cloning Degrees.

Degree of Cloning $=4, D=10 \mathrm{~ms}, L=150$ Bytes, $S_{A}=10 \mathrm{~ms}, S_{B}=15 \mathrm{~ms}$ 


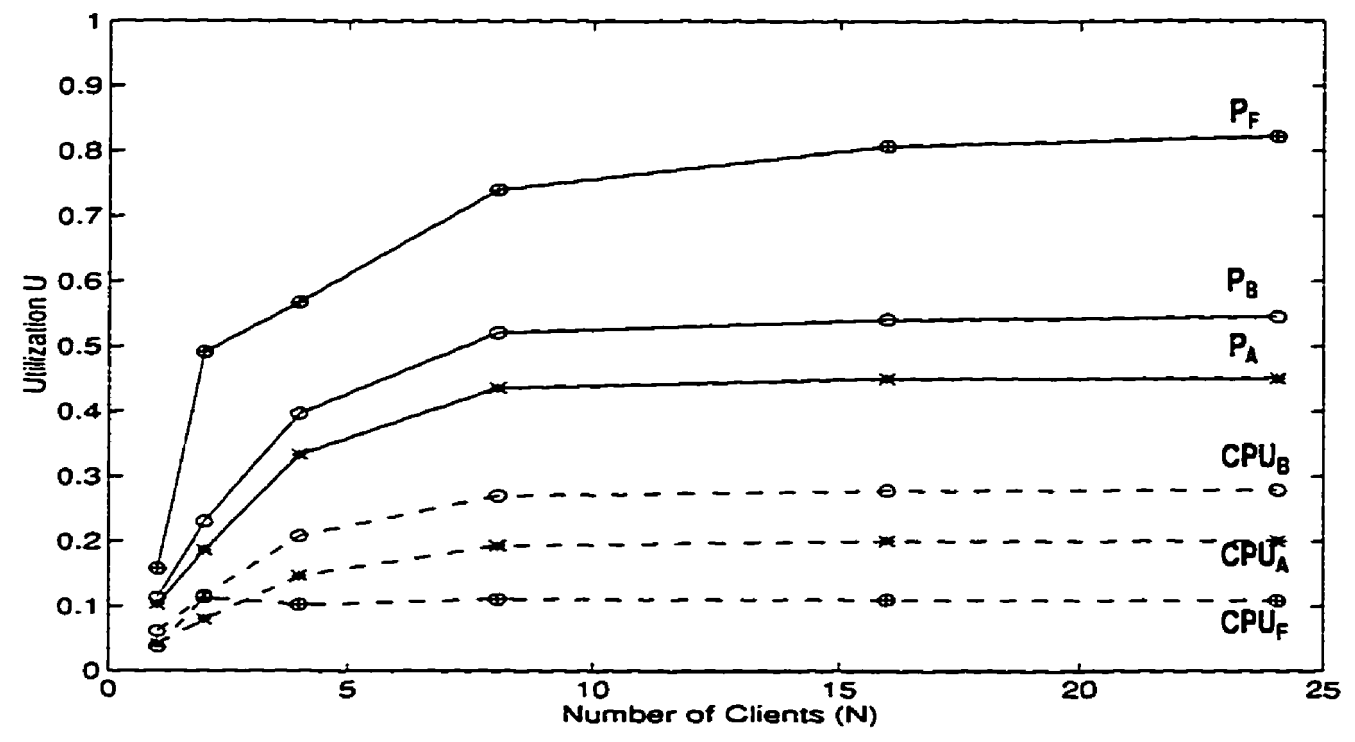

Figure 5-34: Cpu \& Process Utilizations of the F-ORB with $S_{A} / S_{B}=10 / 15 \mathrm{~ms}$, and 4 Cloning Degrees.

$P_{A}=$ Process Util. of Server $A, C P U_{A}=C$ pu Util of Server $A, P_{F}=$ Process Util. of $f$-agent. Degree of Cloning $=4, D=10 \mathrm{~ms}, \hat{\mathrm{L}}=150$ Bytes, $\mathrm{S}_{A}=10 \mathrm{~ms}, \mathrm{~S}_{B}=15 \mathrm{~ms}$

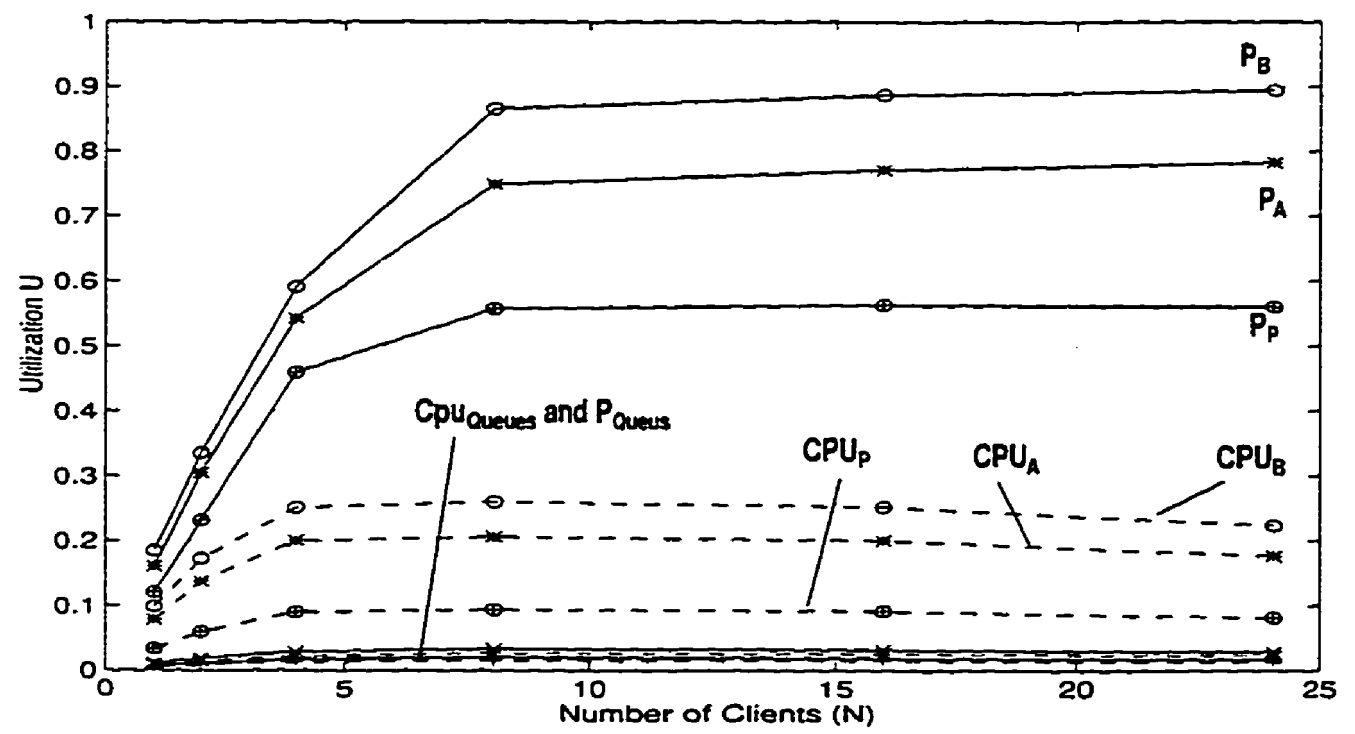

Figure 5-35: Cpu \&Process Utilizations of the P-ORB with $S_{A} / S_{B}=10 / 15 m s$, and 4 Cloning Degrees.

$\mathrm{P}_{\mathrm{A}}=$ Process Util. of Server $\mathrm{A}, \mathrm{CPU} \mathrm{A}_{\mathrm{A}}=\mathrm{Cpu}$ Util of Server $\mathrm{A}, \mathrm{P}_{\mathrm{P}}=$ Process Util. of $\mathrm{p}$-agent. $P_{\text {Queues }}=$ Process Util. of dispatcher, Degree of Cloning $=4, D=10 \mathrm{~ms}, L=150$ Bytes, $\mathrm{S}_{\mathrm{A}}=10 \mathrm{~ms}, \mathrm{~S}_{\mathrm{B}}=15 \mathrm{~ms}$ 


\subsubsection{Eight-Degree Cloned Architectures:}

Following the same approach in Subsection 5.2.4.2, the H-ORB maintains its nocloning status. Hence each of the F-ORB and P-ORBs has eight $f$-agents and eight p-agents respectively in addition to the default agent. The increase in the number of agents reduces the queueing delays on them.

Class A: $10 \mathrm{~ms}$, Class B: $15 \mathrm{~ms}$ - Similar behavioral patterns to the corresponding case of the 4-degree cloned architectures were observed. The related figures are Figure B-14 to Figure B-17 in Appendix B.

Class A: 50 ms, Class B: 75 ms - Similar behavioral patterns to the corresponding case of the 4-degree cloned architectures were observed. The related figures are Figure B-18 to Figure B-21 in Appendix B.

Class A: 250 ms, Class B: 375 ms - Similar behavioral patterns to the corresponding case of the 4-degree cloned architectures were observed. The related figures are Figure B22 to Figure B-25 in Appendix B. A final word on the impact of the server demand is that going from 4 to 8 degrees of cloning does not seem to have a tangible effect on the overall system throughput due to the early saturation of server B which limits any further performance improvement. 


\subsubsection{The Effect of Inter-Node Delay}

Client, server and agent nodes can be located several nodes away from each other. This set of experiments is designed to investigate the relative performance of the three main architectures as the inter-node delay (as the dominant factor) increases under a variable number of $f$-agents and p-agents. Values of the main parameters used in the experiment are listed in Table 16 with the variable parameters in italic fonts. It should be mentioned that the sender process sleeps before sending to simulate the inter-node delay. The F-ORB.VI and V2 were run for all the ranges of inter-node delays and degrees of cloning and their behavior were within $\pm 5 \%$ difference.

Table 16: Parameters of the Inter-Node Delay Experiments

\begin{tabular}{|c|c|}
\hline Main Parameters & Range of values \\
\hline Number of Clients & $1,2,4,816,24$ \\
\hline Service Times & $\begin{array}{l}\text { Class A server }=10 \mathrm{~ms} \\
\text { Class B server }=15 \mathrm{~ms}\end{array}$ \\
\hline Inter-Node Delay & $250 \mathrm{~ms}, 500 \mathrm{~ms}, 1000 \mathrm{~ms}$ \\
\hline Message size & 150 Bytes \\
\hline $\begin{array}{l}\text { Degree of Cloning } \\
\text { H-ORB Arch } \\
\text { F-ORB Arch. } \\
\text { P-ORB Arch. }\end{array}$ & $\begin{array}{l}\text { Default Agent }=1 \\
\text { Default Agent }=1, F \text {-agents }=1,4,8 \\
\text { Default Agent }=1, P \text {-agents }=1,4,8\end{array}$ \\
\hline
\end{tabular}

Therefore, due to the large number of graphs, only one case study of V2 (no cloning, $\mathrm{D}=250 \mathrm{~ms}$ ) is included in Appendix C in Figure C-1 to Figure C-3. Therefore, all the other graphs represent the behavior of the F-ORB.V1. 


\subsubsection{No Cloning (Single Agent) Architectures:}

Inter-Node Delay $250 \mathrm{~ms}$ - The influence of the inter-node delay as the number of clients increases on the mean client response time and the corresponding overall system throughput is studied at low to high levels of system loading.

\section{Low Load Conditions:}

As captured in Table 17, Figure 5-36 and Figure 5-37, when $N=1$, the P-ORB has the best latency due to the concurrent invocation of the servers which reduces the total number of inter-node delays as well as the absence of any queueing delays. The F-ORB comes second due to its fewer number of delays compared with the $\mathrm{H}-\mathrm{ORB}$. When $\mathrm{N}$ is 2 , both the F-ORB and the P-ORB exhibit identical behavior and the reason for that is the fact that the gain obtained from the concurrent invocation of the servers is lost by the queueing delay faced by the server returning the reply to the p-agent.

Table 17: Effect of $D$ on Latency at $N=1\left(L=150\right.$ bytes, $S_{A} / S_{B}=10 / 15$ ms, no Cloning)

\begin{tabular}{|c|c|c|c|}
\hline D (ms) & H-ORB (sec) & F-ORB (sec) & P-ORB (sec) \\
\hline 250 & 2.0902 & 1.5928 & 1.0923 \\
\hline 500 & 4.0986 & 3.0952 & 2.0935 \\
\hline 1000 & 8.0974 & 6.0971 & 4.0916 \\
\hline
\end{tabular}

\section{Medium to High Load Conditions:}

When $\mathrm{N}$ goes beyond 4, the $\mathrm{H}-\mathrm{ORB}$ demonstrates a much superior performance in comparison with the other two architectures. The reason for this behavior is the software 


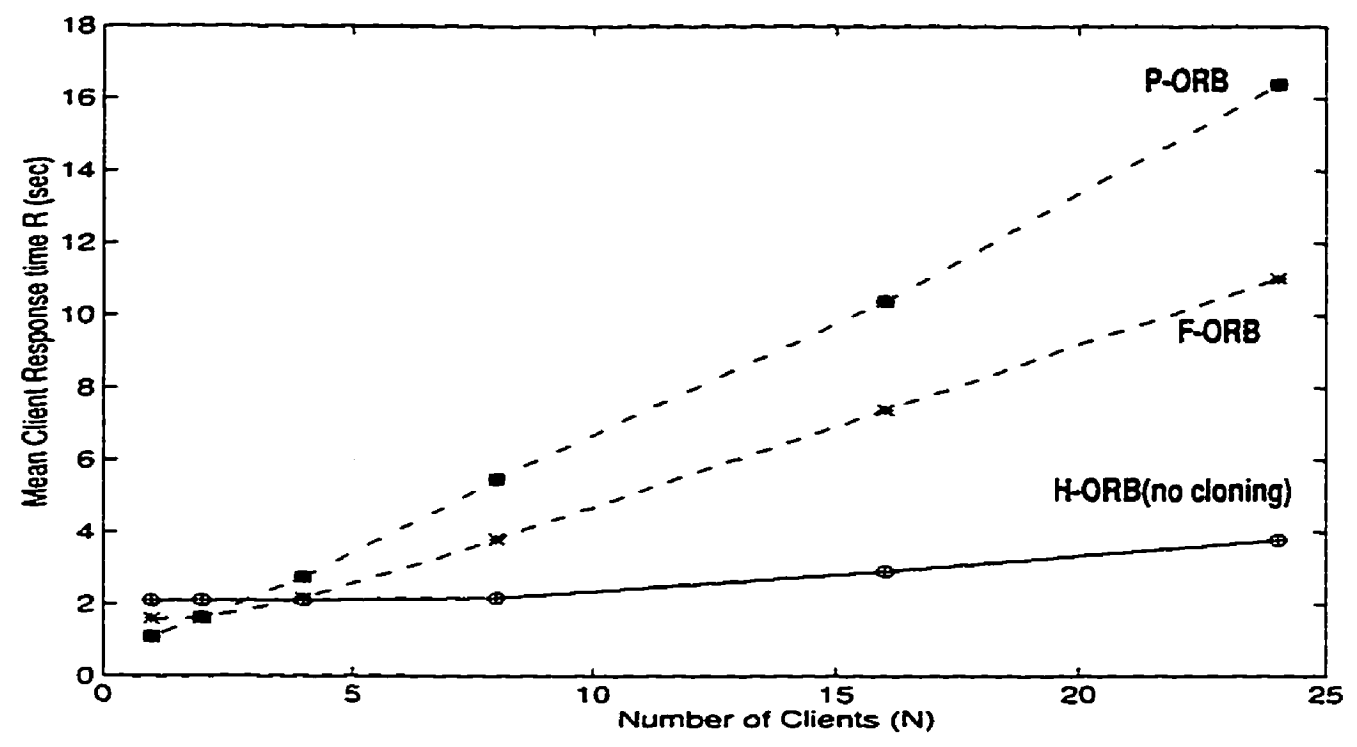

Figure 5-36: The Response Time of the H-ORB, the F-ORB and the PORB with $D=250 \mathrm{~ms}$, and no Cloning.

Degree of Cloning $=1, D=250 \mathrm{~ms}, L=150$ Bytes, $S_{A}=10 \mathrm{~ms}, S_{B}=15 \mathrm{~ms}$

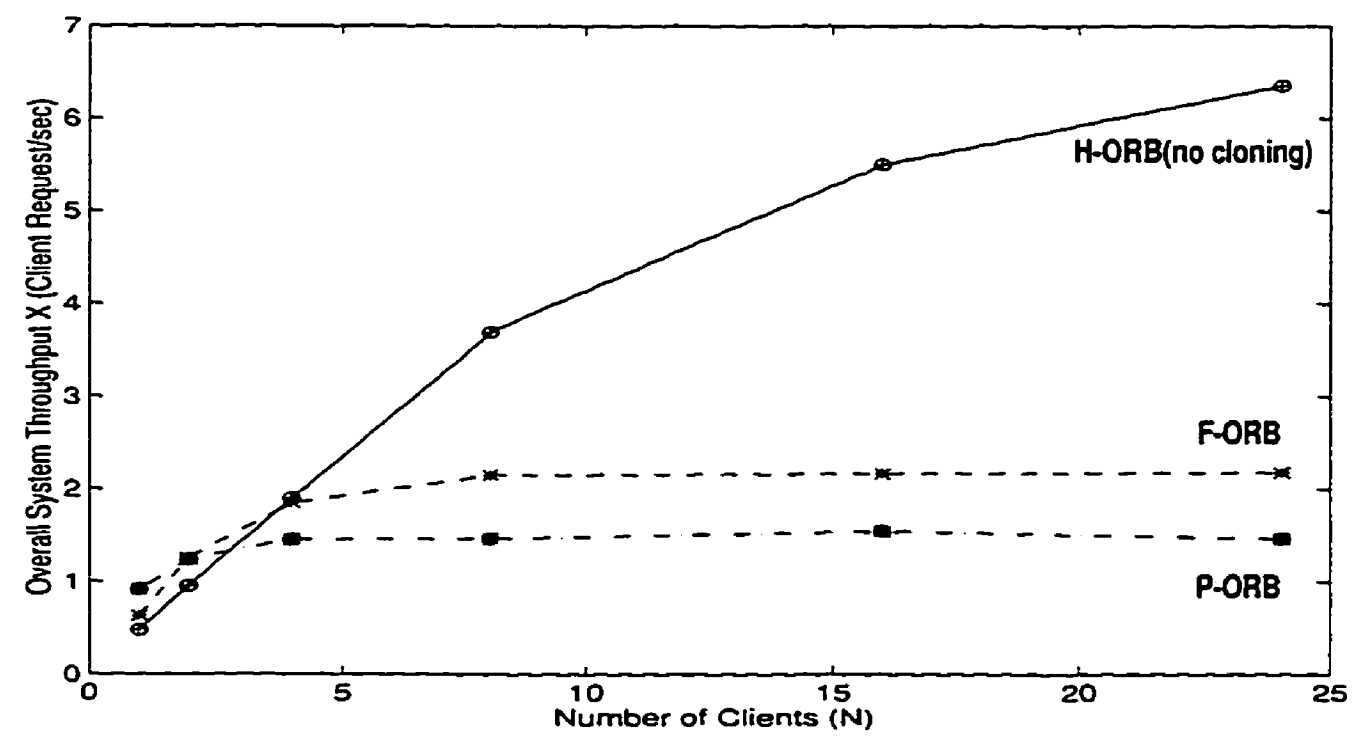

Figure 5-37: The Throughput of the H-ORB, the F-ORB and the P-ORB with $D=250 \mathrm{~ms}$, and no Cloning.

Degree of Cloning= 1, D $=250 \mathrm{~ms}, L=150$ Bytes, $S_{A}=10 \mathrm{~ms}, S_{B}=15 \mathrm{~ms}$ 


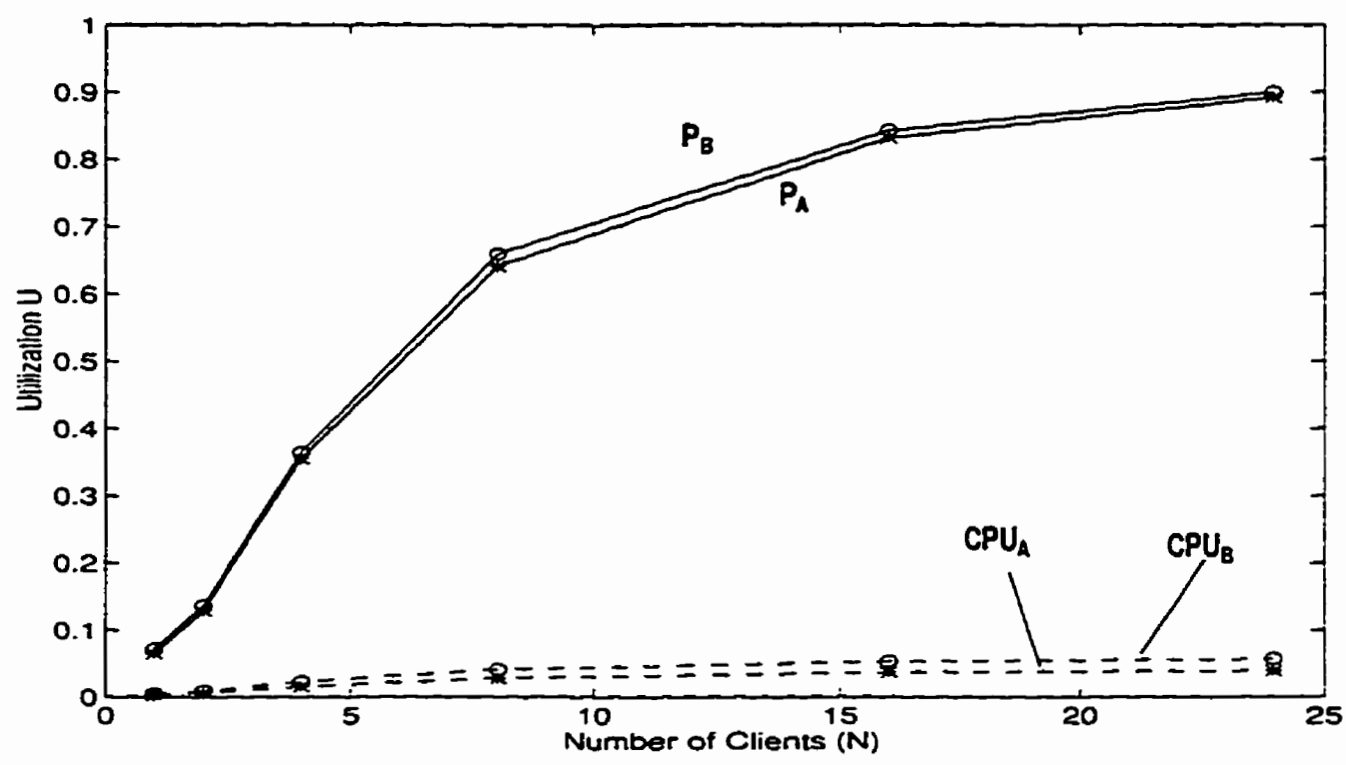

Figure 5-38: Cpu and Process Utilizations of the H-ORB with $D=250 \mathrm{~ms}$, and no Cloning.

$P_{A}=$ Process Util. of Server $A, C P U_{A}=C$ pu Util of Server $A$

Degree of Cloning $=1, D=250 \mathrm{~ms}, L=150$ Bytes, $S_{A}=10 \mathrm{~ms}, S_{B}=15 \mathrm{~ms}$

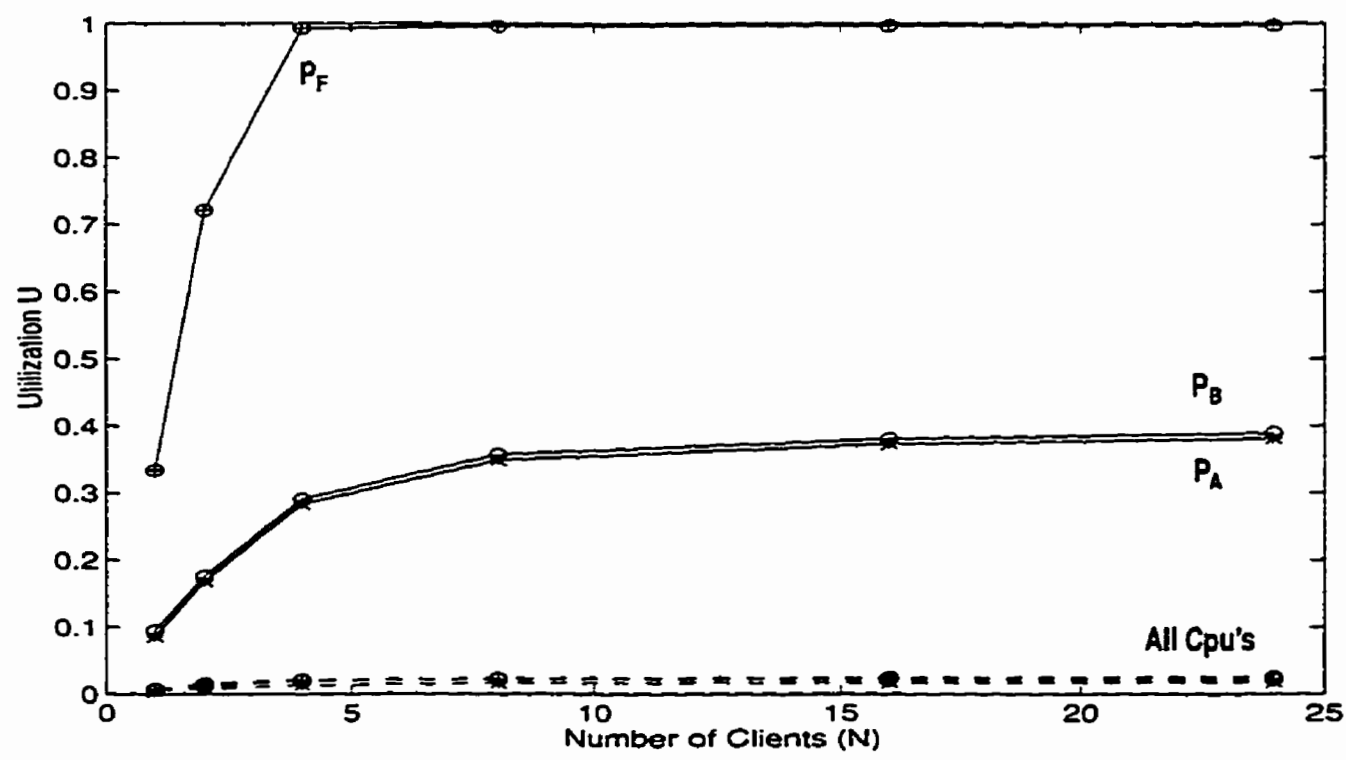

Figure 5-39: Cpu \&Process Utilizations of the F-ORB with $D=250 \mathrm{~ms}$, and no Cloning.

$P_{A}=$ Process Util. of Server $A, C P U_{A}=C$ pu Util of Server $A, P_{F}=$ Process Util. of f-agent.

Degree of Cloning $=1, D=250 \mathrm{~ms}, \mathrm{~L}=150$ Bytes, $S_{A}=10 \mathrm{~ms}, S_{B}=15 \mathrm{~ms}$ 


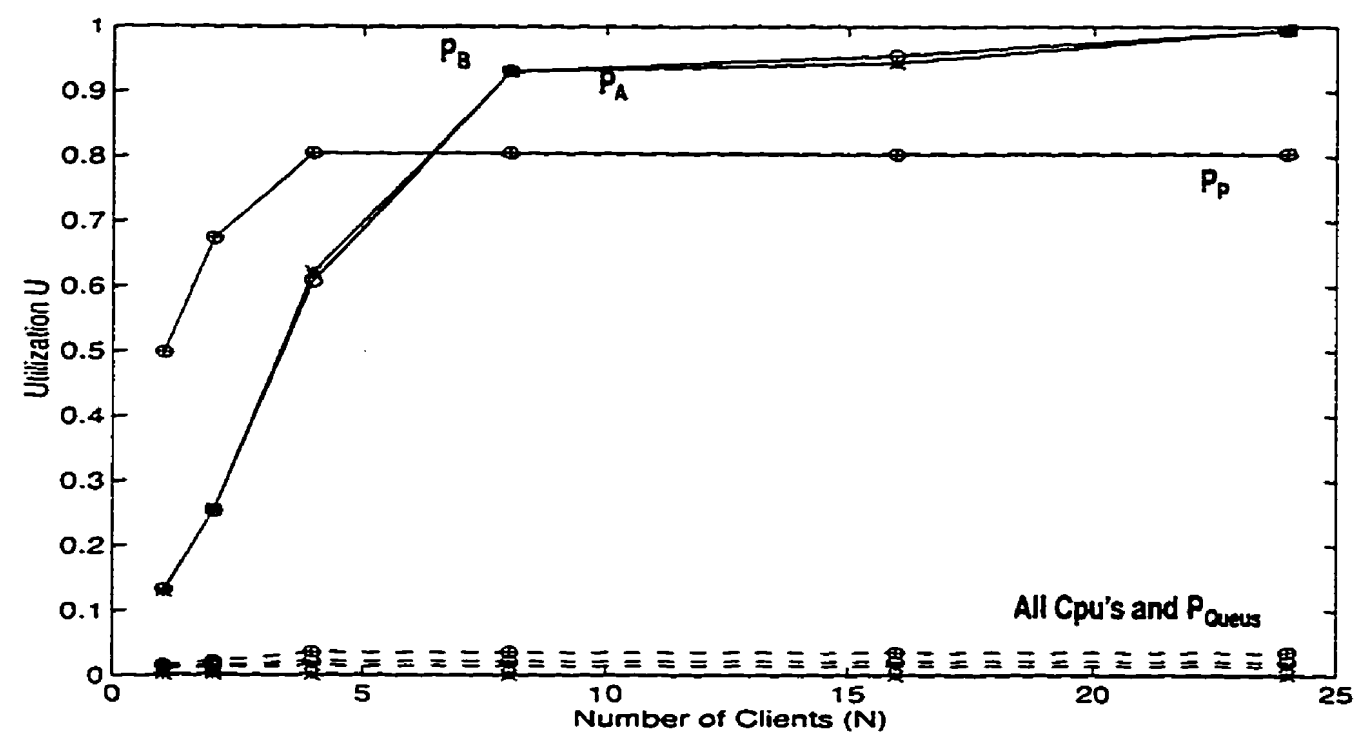

Figure 5-40: Cpu \&Process Utilizations of the P-ORB with $D=250 \mathrm{~ms}$, and no Cloning Degrees.

$P_{A}=$ Process Util. of Server $A, C P U_{A}=C$ pu Util of Server $A, P_{P}=$ Process Util. of p-agent. $P_{\text {Queues }}=$ Process Util. of dispatcher, Degree of Cloning $=1, D=250 \mathrm{~ms}, L=150$ Bytes, $\mathrm{S}_{\mathrm{A}}=10 \mathrm{~ms}, \mathrm{~S}_{\mathrm{B}}=15 \mathrm{~ms}$

bottlenecking phenomenon described in detail in Subsection 5.2.4.1. The related utilization graphs in Figure 5-39 shows that the f-agent is saturating around $N=8$, whereas in the $P$ ORB utilization graphs of Figure 5-40, both servers are saturating. (in this case study, both server are saturating since their service times are much smaller than the simulated intermode delays).

Inter-Node Delay 500 ms - Identical behavioral patterns were observed under the whole range of request loads as shown in Appendix C in Figure C-4 to Figure C-8. However, as expected, longer mean client response time and lower overall system throughput were observed. Table 17 and Table 18 show the latency when $\mathrm{N}=1$ and the maximum throughput at $N=24$, respectively, for the three architectures for all values of $D$. 
Table 18: Throughput at $N=24\left(L=150\right.$ bytes, $S_{A} / S_{B}=10 / 15$ ms, no Cloning)

\begin{tabular}{|c|c|c|c|}
\hline $\begin{array}{c}\mathbf{D} \\
(\mathrm{ms})\end{array}$ & $\begin{array}{c}\text { H-ORB } \\
\text { (Client Request/sec) }\end{array}$ & $\begin{array}{c}\text { F-ORB } \\
\text { (Client Request/sec) }\end{array}$ & $\begin{array}{c}\text { P-ORB } \\
\text { (Client Reques/sec) }\end{array}$ \\
\hline 250 & 6.3525 & 2.1773 & 1.4637 \\
\hline 500 & 3.3100 & 1.1283 & 0.8248 \\
\hline 1000 & 1.7449 & 0.5788 & 0.3904 \\
\hline
\end{tabular}

Inter-Node Delay $1000 \mathrm{~ms}$ - Identical behavioral patterns were also observed as shown in Appendix C in Figure C-9 to Figure C-13. However, as expected, the mean client response time were the longest to be measured and minimum values for throughput were observed.

\subsubsection{Four-Degree Cloned Architectures:}

The cloning approach is also used to alleviate the software bottlenecks encountered in the no-cloning case study. As explained in Subsection 5.2.4.2, the H-ORB retains its nocloning status while each of the F-ORB and P-ORB will have four f-agents and four pagents respectively, in addition to the default agent.

Inter-Node Delay 250 ms - Figure 5-41 and Figure 5-42 capture the mean client response time and the corresponding overall system throughput respectively. The analysis of these figures reveals that the cloning has significantly improved the overall system throughput for the F-ORB and the P-ORB architectures due to the load distribution on the four agents and the consequent reduction of the queueing time at the agents for all incoming requests/replies. Also, as a result of this improvement in performance, the crossover point 
of the H-ORB with the other two architectures has been shifted to higher values of $\mathrm{N}$ than that of the corresponding no-cloning case. The P-ORB supremacy is due to the effect of the pseudo asynchronous parallel calls, used when invoking the servers.

Table 19: Effect of $D$ on Latency at $N=1\left(L=150\right.$ bytes, $S_{A} / S_{B}=10 / 15 \mathrm{~ms}, 4$ Cloning Degrees)

\begin{tabular}{|c|c|c|c|}
\hline D (ms) & $\begin{array}{c}\text { H-ORB (sec) } \\
\text { (no cloning) }\end{array}$ & F-ORB (sec) & P-ORB (sec) \\
\hline 250 & 2.0902 & 1.6007 & 1.0923 \\
\hline 500 & 4.0986 & 3.0899 & 2.0935 \\
\hline 1000 & 8.0974 & 6.0899 & 4.0916 \\
\hline
\end{tabular}

Inter-Node Delay $500 \mathrm{~ms}$ - Identical behavioral patterns were observed under the whole range of request loads as shown in Appendix C in Figure C-14 to Figure C-17. However, as expected, longer mean client response time and lower throughput were measured. Table 19 and Table 20 show the latency when $\mathrm{N}=1$ and the maximum throughput at $\mathrm{N}=24$, respectively, for the three architectures.

Table 20: Throughput at $N=24\left(L=150\right.$ bytes, $S_{A} / S_{B}=10 / 15$ ms, 4 Cloning Degrees)

\begin{tabular}{|c|c|c|c|}
\hline $\begin{array}{c}\text { D } \\
\text { (ms) }\end{array}$ & $\begin{array}{c}\text { H-ORB } \\
\text { (no cloning) } \\
\text { (Client Request/sec) }\end{array}$ & $\begin{array}{c}\text { F-ORB } \\
\text { (Client Request/sec) }\end{array}$ & $\begin{array}{c}\text { P-ORB } \\
\text { (Client Requestsec) }\end{array}$ \\
\hline 250 & 6.3525 & 4.2103 & 1.4637 \\
\hline 500 & 3.3100 & 2.2572 & 0.8248 \\
\hline 1000 & 1.7449 & 1.1619 & 0.3904 \\
\hline
\end{tabular}




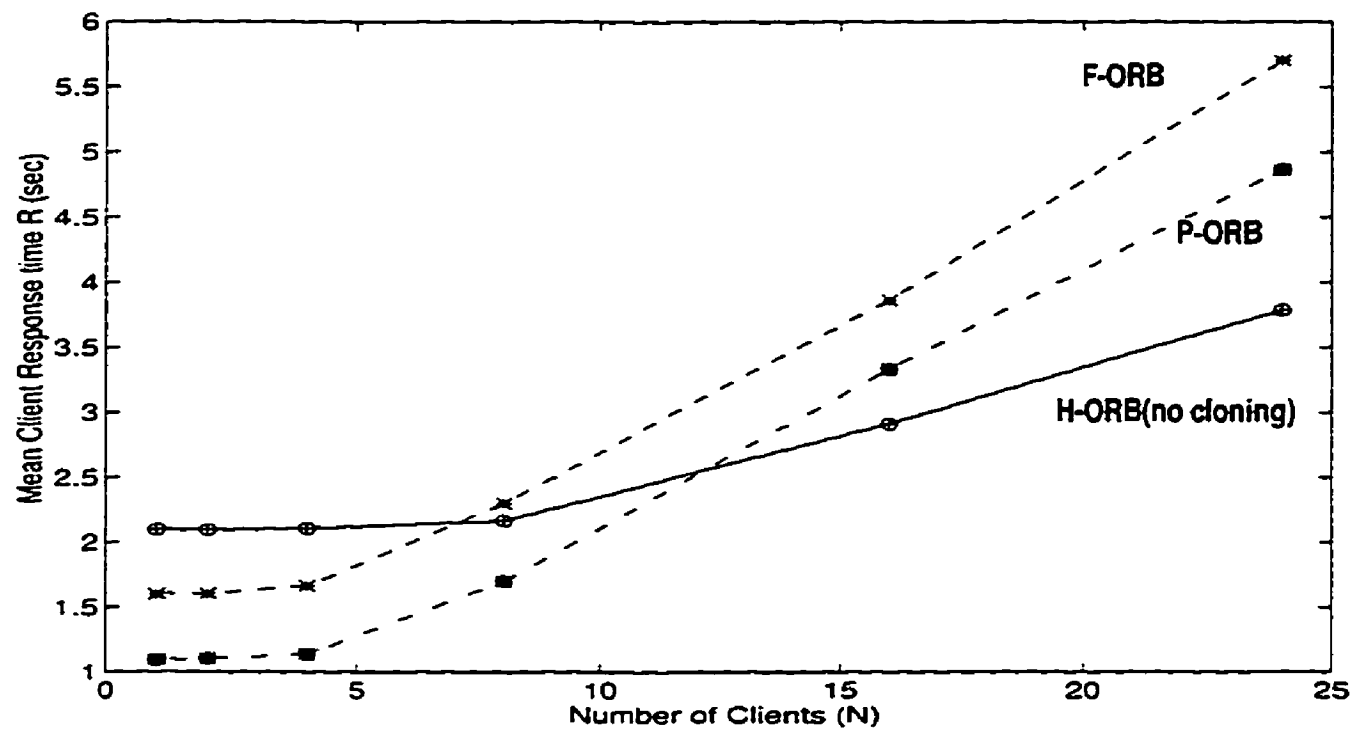

Figure 5-41: The Response Time of the H-ORB, the F-ORB and the PORB with $D=250 \mathrm{~ms}$, and 4 Cloning Degrees.

Degree of Cloning $=4, D=250 \mathrm{~ms}, L=150$ Bytes, $S_{A}=10 \mathrm{~ms}, S_{B}=15 \mathrm{~ms}$

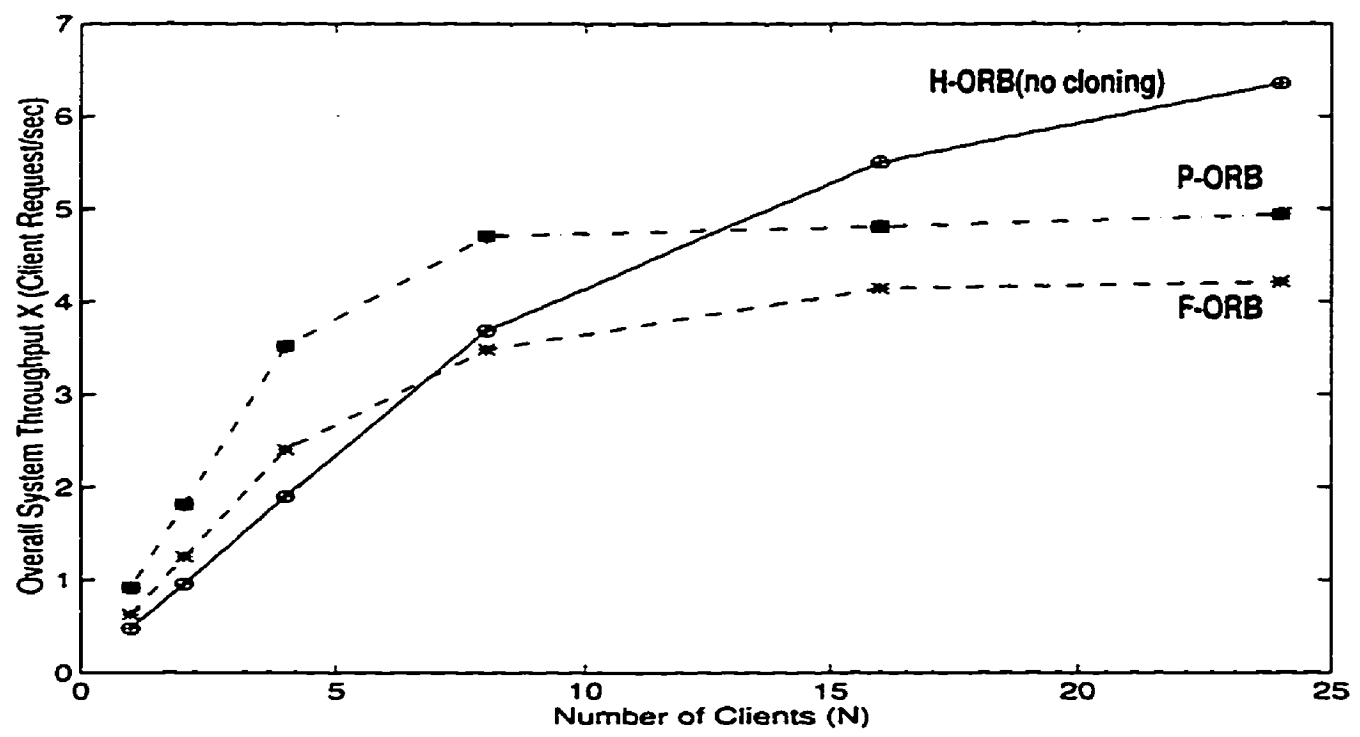

Figure 5-42: The Throughput of the H-ORB, the F-ORB and the P-ORB with $D=250 \mathrm{~ms}$, and 4 Cloning Degrees.

Degree of Cloning $=4, D=250 \mathrm{~ms}, L=150$ Bytes, $S_{A}=10 \mathrm{~ms}, S_{B}=15 \mathrm{~ms}$ 


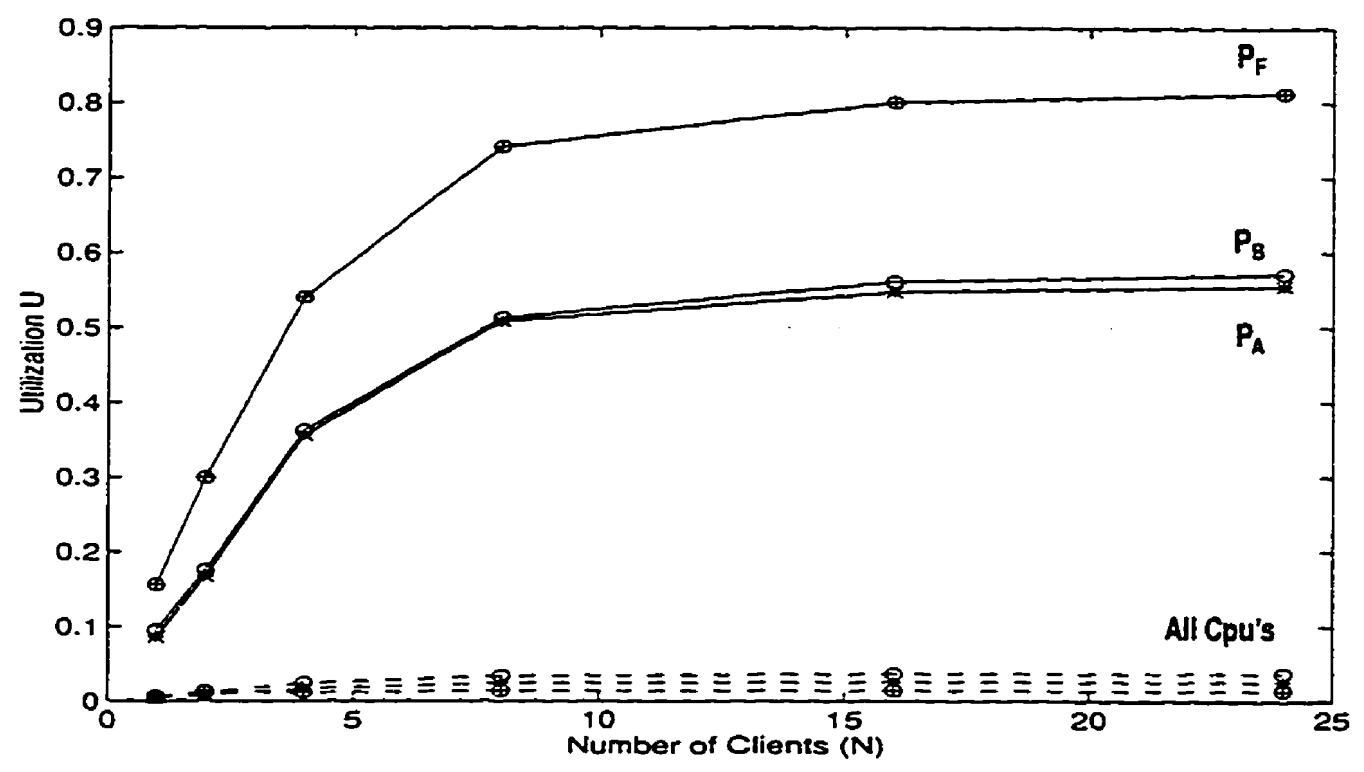

Figure 5-43: Cpu \&Process Utilizations of the F-ORB with $D=250 \mathrm{~ms}$, and 4 Cloning Degrees.

$P_{A}=$ Process Util. of Server $A, C P U_{A}=C p u$ Util of Server $A, P_{F}=$ Process Util. of f-agent. Degree of Cloning $=4, D=250 \mathrm{~ms}, L=150$ Bytes, $S_{A}=10 \mathrm{~ms}, S_{B}=15 \mathrm{~ms}$

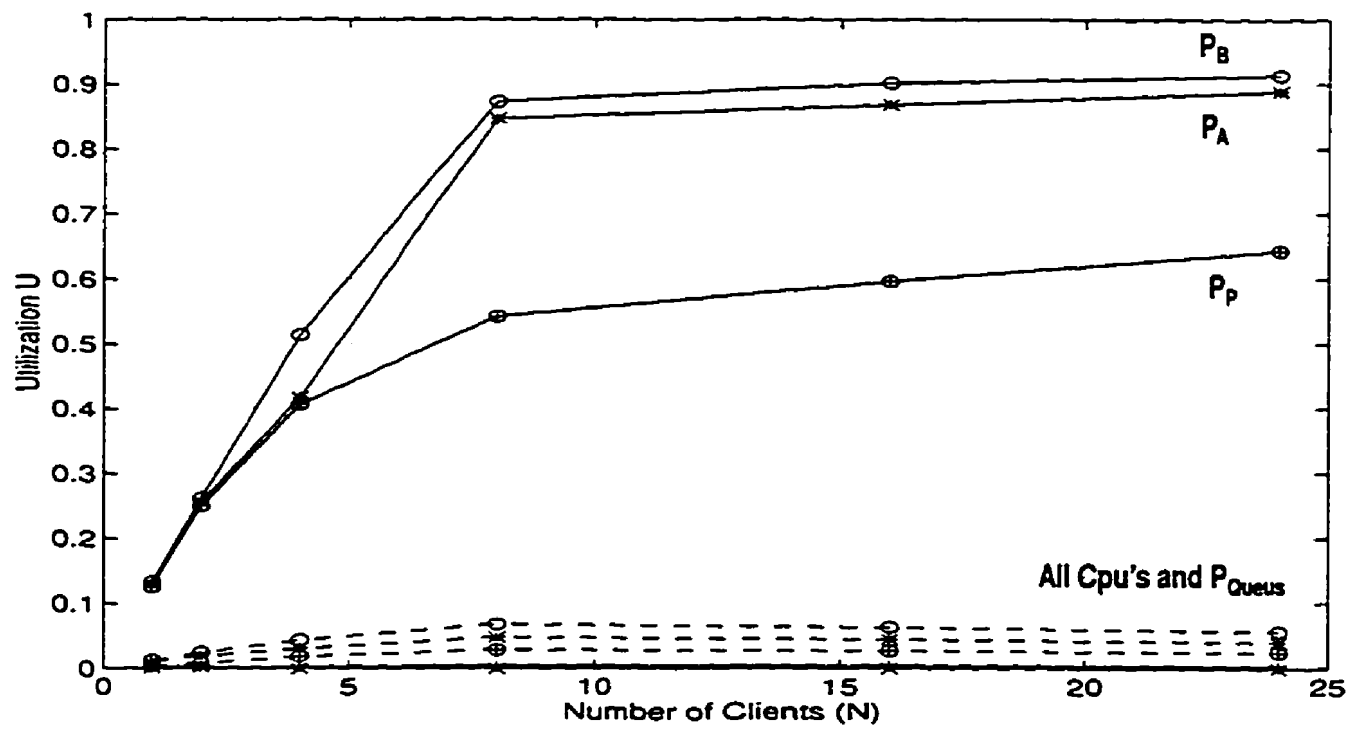

Figure 5-44: Cpu \& Process Utilizations of the P-ORB with $D=250 \mathrm{~ms}$, and 4 Cloning Degrees.

$P_{A}=$ Process Util. of Server $A, C P U_{A}=C p u$ Util of Server $A, P_{P}=$ Process Util. of p-agent. $P_{\text {Queves }}=$ Process Util. of dispatcher, Degree of Cloning $=4, D=250 \mathrm{~ms}, L=150$ Bytes, $\mathrm{S}_{\mathrm{A}}=10 \mathrm{~ms}, \mathrm{~S}_{\mathrm{B}}=15 \mathrm{~ms}$ 
Inter-Node Delay $1000 \mathrm{~ms}$ - Identical behavioral patterns were also observed in Appendix C in Figure C-18 to Figure C-21. However, as expected, the mean client response time was the longest to be measured. Thus, the corresponding throughput was minimized.

\subsubsection{Eight-Degree Cloned Architectures:}

Following the same approach in Subsection 5.2.4.2, the H-ORB maintains its nocloning status. Hence each of the F-ORB and P-ORBs has eight $f$-agents and eight $p$-agents respectively in addition to the default agent. The increase in the number of agents reduces the queueing delays on the agents.

Table 21: Effect of $D$ on Latency at $N=1\left(L=150\right.$ bytes, $S_{A} / S_{B}=10 / 15 \mathrm{~ms}, 8$ Cloning Degrees)

\begin{tabular}{|c|c|c|c|}
\hline$D(m s)$ & $\begin{array}{c}\text { H-ORB (sec) } \\
\text { (no cloning) }\end{array}$ & F-ORB (sec) & P-ORB (sec) \\
\hline 250 & 2.0902 & 1.5972 & 1.0942 \\
\hline 500 & 4.0986 & 3.1036 & 2.0972 \\
\hline 1000 & 8.0974 & 6.0912 & 4.0981 \\
\hline
\end{tabular}

Inter-Node Delay $250 \mathrm{~ms}$ - Figure 5-41 and Figure 5-42 capture the mean client response time and the corresponding overall system throughput respectively. The analysis of these figures reveals that the cloning has significantly improved the overall system throughput for the F-ORB and the P-ORB architectures due to the load distribution on the four agents and the consequent reduction of the queueing time at the agents for all incoming requests/replies. Also, as a result of this improvement in performance, the crossover point of the $\mathrm{H}-\mathrm{ORB}$ with the F-ORB architecture has been shifted to higher values of $\mathrm{N}$ compared 


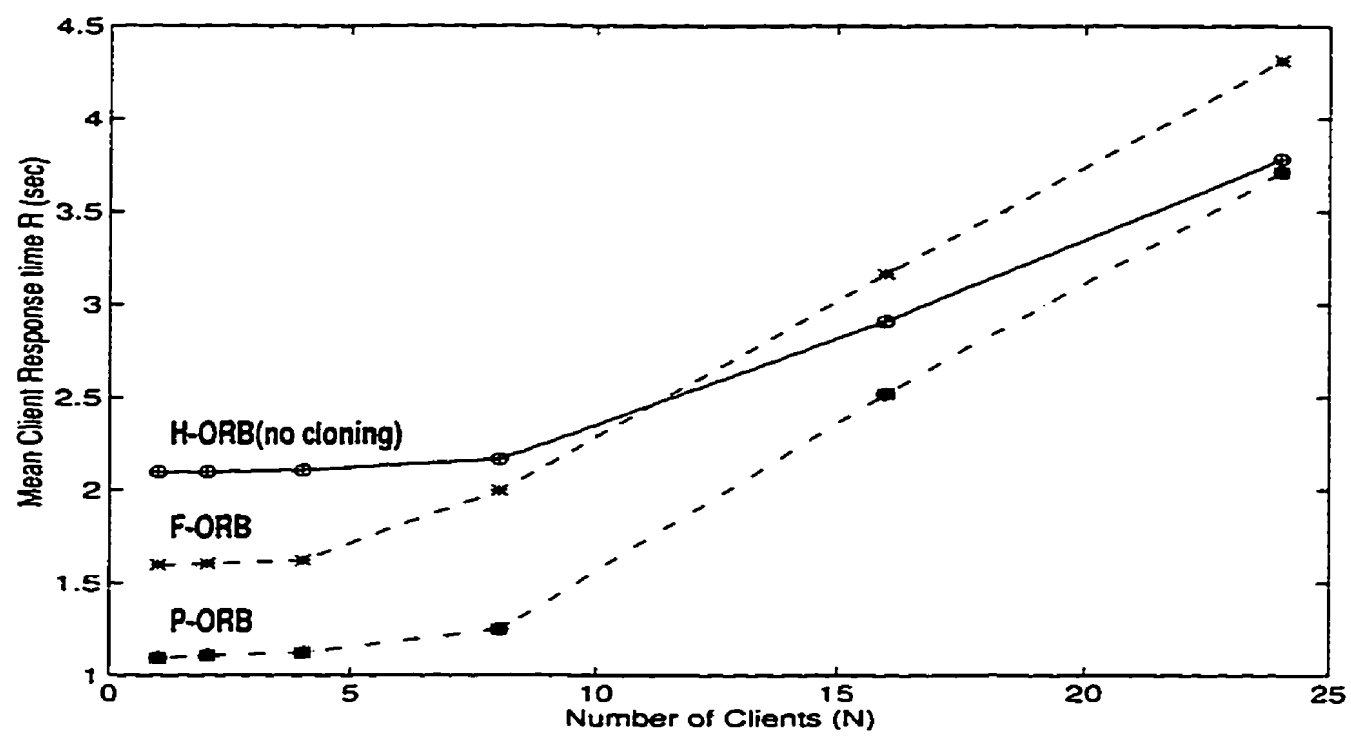

Figure 5-45: The Response Time of the H-ORB, the F-ORB and the P$O R B$ with $D=250 \mathrm{~ms}$, and 8 Cloning.

Degree of Cloning $=8, D=250 \mathrm{~ms}, L=150$ Bytes, $S_{A}=10 \mathrm{~ms}, S_{B}=15 \mathrm{~ms}$

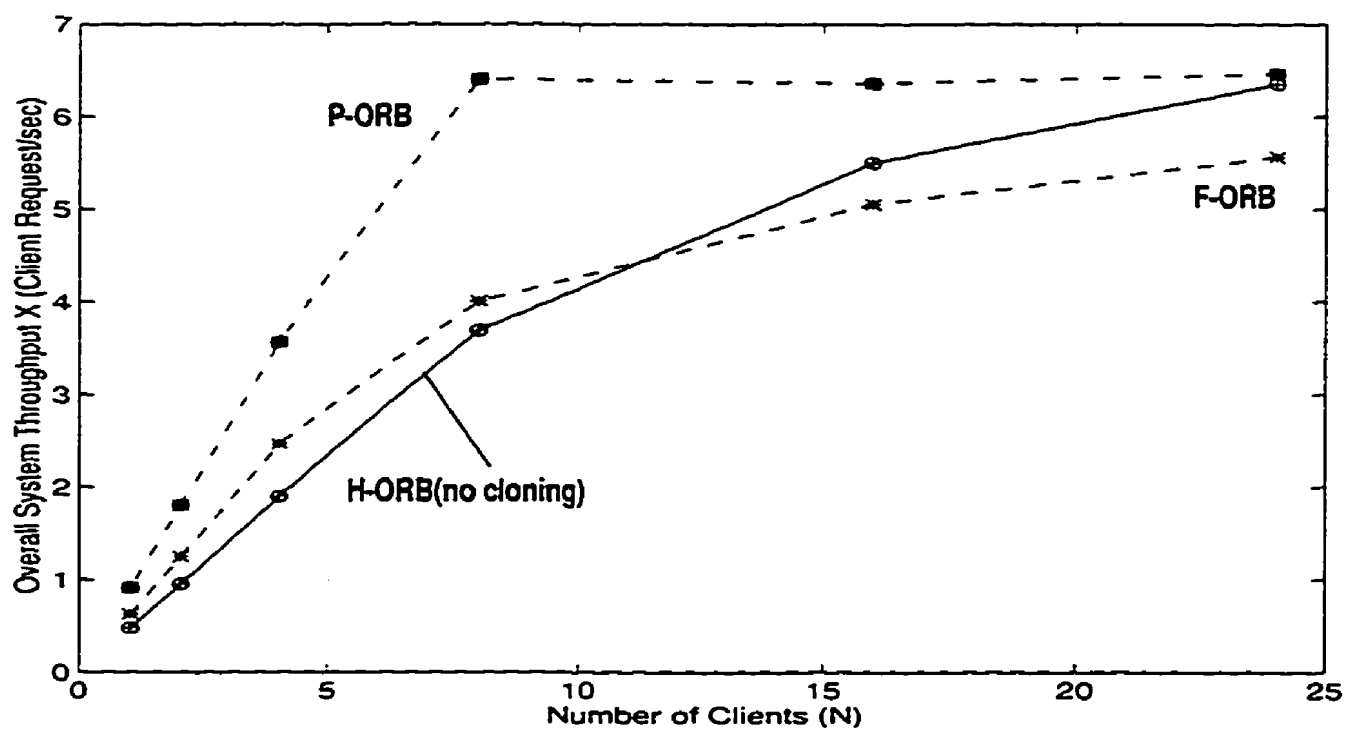

Figure 5-46: The Throughput of the H-ORB, the F-ORB and the P-ORB with $D=250 \mathrm{~ms}$, and 8 Cloning.

Degree of Cloning $=8, D=250 \mathrm{~ms}, L=150$ Bytes, $S_{A}=10 \mathrm{~ms}, S_{B}=15 \mathrm{~ms}$ 


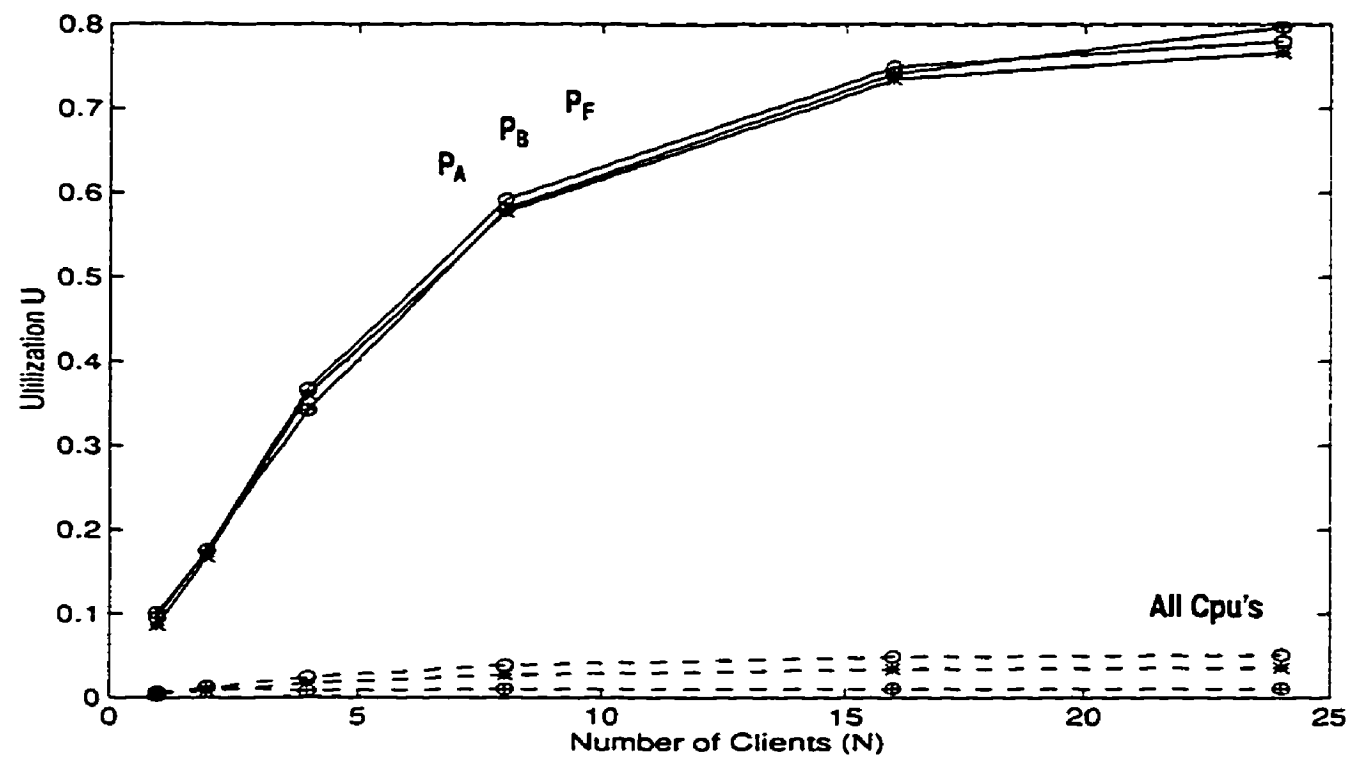

Figure 5-47: Cpu \& Process Utilizations of the F-ORB with D $=250 \mathrm{~ms}$, and 8 Cloning Degrees.

$P_{A}=$ Process Util. of Server $A, C P U_{A}=C$ pu Util of Server $A, P_{F}=$ Process Util. of $f$-agent. Degree of Cloning $=8, D=250 \mathrm{~ms}, L=150$ Bytes, $S_{A}=10 \mathrm{~ms}, S_{B}=15 \mathrm{~ms}$

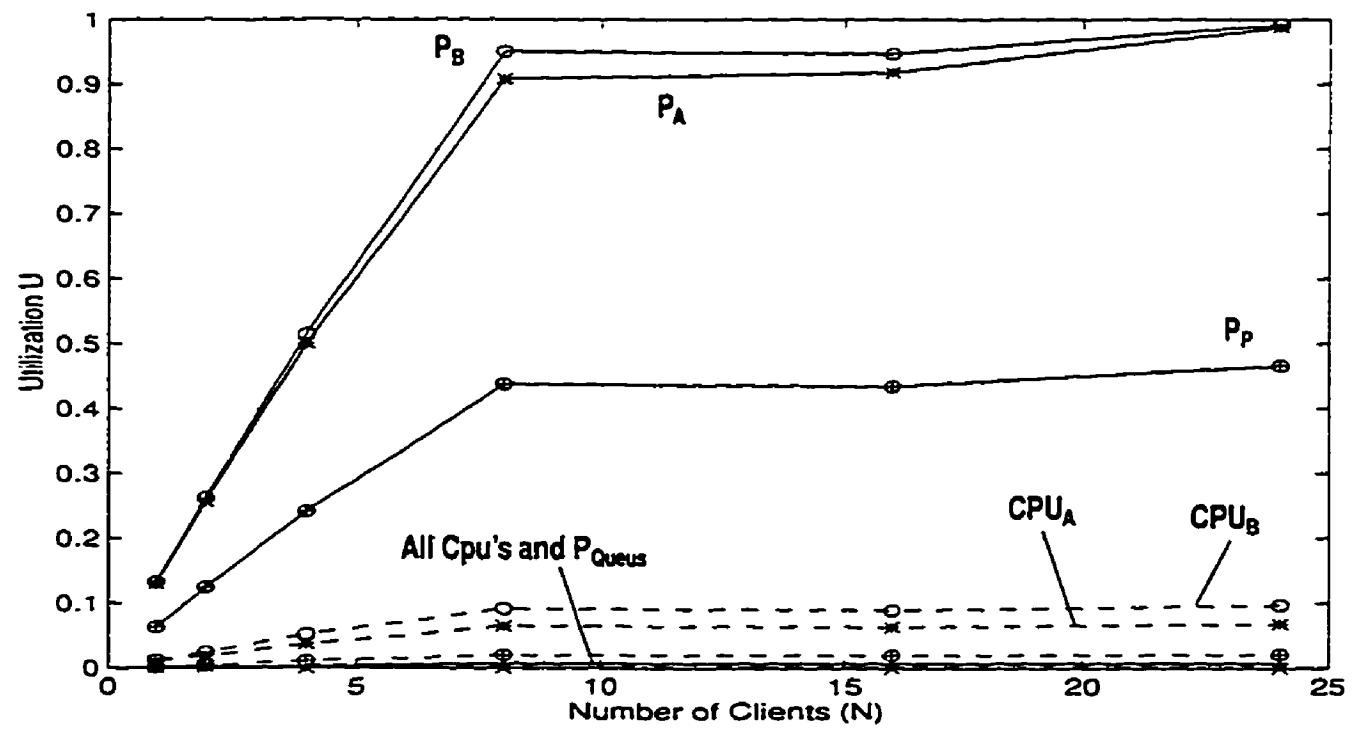

Figure 5-48: Cpu \&Process Utilizations of the $P-O R B$ with $D=250 \mathrm{~ms}$, and 8 Cloning Degrees.

$\mathrm{P}_{\mathrm{A}}=$ Process Util. of Server $\mathrm{A}, C P U_{\mathrm{A}}=\mathrm{Cpu}$ Util of Server $\mathrm{A}, \mathrm{P}_{\mathrm{p}}=$ Process Util. of p-agent. $P_{\text {Queues }}=$ Process Util. of dispatcher, Degree of Cloning $=8, D=250 \mathrm{~ms}, L=150$ Bytes, $\mathrm{S}_{\mathrm{A}}=10 \mathrm{~ms}, \mathrm{~S}_{\mathrm{B}}=15 \mathrm{~ms}$ 
with that of the respective 4-degree cloned case. The P-ORB supremacy is due to the effect of the pseudo asynchronous parallel calls, used when invoking the servers and the reduction in the agent queueing time.

Inter-Node Delay $500 \mathrm{~ms}$ - Similar behavioral patterns were observed as illustrated in Appendix $C$ in Figure C-22 to Figure C-25. However, it is noticed that the H-ORB crossover point is shifted to the left near $\mathrm{N}=16$ because the larger the inter-node delays the more the effect of the F-ORB shorter path. Also, as expected, the values of the response time are longer than their respective values at the $250 \mathrm{~ms}$ delay case study and consequently the overall throughput is reduced.

Table 22: Throughput at $N=24\left(L=150\right.$ bytes, $S_{A} / S_{B}=10 / 15$ ms, 8 Cloning Degrees)

\begin{tabular}{|c|c|c|c|}
\hline $\begin{array}{c}\text { D } \\
(\mathrm{ms})\end{array}$ & $\begin{array}{c}\text { H-ORB } \\
\text { (no cloning) } \\
\text { (Client Requestsec) }\end{array}$ & $\begin{array}{c}\text { F-ORB } \\
\text { (Client Reques/sec) }\end{array}$ & $\begin{array}{c}\text { P-ORB } \\
\text { (Client Request/sec) }\end{array}$ \\
\hline 250 & 6.3525 & 5.5612 & 6.4616 \\
\hline 500 & 3.3100 & 3.2446 & 3.5640 \\
\hline 1000 & 1.7449 & 1.6180 & 1.8308 \\
\hline
\end{tabular}

Inter-Node Delay $1000 \mathrm{~ms}$ - Similar behavioral patterns were observed as illustrated in Appendix C in Figure C-26 to Figure C-29. However, it is noticed that the H-ORB crossover point is shifted further to the left near $\mathrm{N}=17$ because the larger the inter-node delays the more the effect of the F-ORB shorter path. Also, as expected, the values of the response time are longer than their respective values at the $250 \mathrm{~ms}$ delay case study and consequently the overall throughput is reduced. 


\subsection{Discussion of Results}

A comprehensive experimental study on the relative performance of the H-ORB, the F$\mathrm{ORB}$ and the P-ORB architectures under different workload parameters and cloning levels was presented in the previous subsections. A summary of the results of the experiments on the impact of the message size, server demand, and inter-node delay is presented.

\subsubsection{The Message Size L}

The effect of message size on system performance is covered in details in Subsection 5.2.4. The throughput of all three architectures deteriorates as the message size increases. This is due to the overhead of marshalling/unmarshalling and the time needed to send the message to the destination node. Both of the F-ORB and the P-ORB behaved poorly due to the software bottleneck effect in the f-agent and server B, respectively, (details in Subsection 5.2.4.1). No software bottlenecks have been identified in the H-ORB.

Another issue that warrants studying is the sensitivity of the three architectures to the increase in the message size (the Architecture Sensitivity in this context is the percentage change in the overall system throughput when a specific parameter is changed from its minimum to its maximum value with a fixed degree of cloning). It has been noted that when L was increased from 4800 to 19200 bytes (no cloning), throughput was reduced by $11.2 \%$, $17.4 \%$ and $5.1 \%$ for the $\mathrm{H}-\mathrm{ORB}$, the F-ORB and the P-ORB respectively. The F-ORB suffered most due to the marshalling/unmarshalling overhead incurred by the six message transfers in each request. The H-ORB was less sensitive since the messages exchanged with the default agent are very short (request/handle) and are not affected by the increase in the 
message size $L$. The P-ORB is least sensitive due to the alleviating effect of the concurrent invocation of the servers which reduces the effect of the inter-node delays as well as the extra marshalling/unmarshalling overhead. This sensitivity pattem holds for higher degrees of cloning.

The cloning of the agent is introduced to counteract the effect of the software bottleneck discovered in the F-ORB and the P-ORB. Significant improvement in the throughput of the F-ORB and the P-ORB has been observed. As a result of this cloning, and in certain cases ( $L=4800, N=24$ ) the improvement was $157 \%$ and $319 \%$ for the $F-O R B$ and the P-ORB respectively when the degree of cloning was increased form 1 to 8 . Consequently, the relative performance of the three architectures has changed. While the P-ORB behaved worst with no cloning, it became the best with 8 cloned agents.

\subsubsection{The Server Demand $S_{A} / S_{B}$}

The impact of server demand on the overall system performance is discussed in details in Subsection 5.2.5. Without agent cloning, the throughputs of all three architectures deteriorate as $\mathrm{S}_{\mathrm{A}}$ and $\mathrm{S}_{\mathrm{B}}$ increase as a result of an increase in the queueing delay and the service time at the server. With small to medium server demands, both the F-ORB and the $\mathrm{P}-\mathrm{ORB}$ gave an inferior performance relative to the $\mathrm{H}-\mathrm{ORB}$. This can be attributed to the software bottlenecking described earlier. However, with large server demands (250/375 ms) the P-ORB performed better taking advantage of the concurrent invocation of the servers.

As for the sensitivity of the three architectures to the increase in the server demands, it has been observed that the throughputs (at $\mathrm{N}=24$ ) were decreased by $91 \%, 82 \%$, and $68 \%$ 
for the $\mathrm{H}-\mathrm{ORB}, \mathrm{F}-\mathrm{ORB}$ and the P-ORB, respectively, when the server demand were increased from (10/15) to (250/375) ms with no cloning. Slightly higher sensitivities were observed at higher degrees of cloning.

Using the same approach as in the message size case study, agent cloning has also been used. A significant increase of $146 \%$ in the F-ORB throughput was observed when the server demands was (10/15 ms) and the cloning degree was increased form 1 to 8 . However, the P-ORB demonstrated only a modest increase due to the overhead of the frequent binding operations. This is because the P-ORB does not store any object reference locally, and all references are obtained dynamically in each invocation. The small value of the internode delay makes the binding effect more dominant. As the sever demands increases, agent cloning becomes less effective for both of the F-ORB and the P-ORB due to the early saturation of class B servers.

\subsubsection{The Inter-Node Delays D}

An in-depth analysis of the effect of inter-node delays on the relative performance of the H-ORB, the F-ORB and the P-ORB is presented in Subsection 5.2.6. With no agent cloning, the throughputs of all three architectures decreased as the inter-node delay increased. As in the previous two cases, the F-ORB and the P-ORB behaved poorly due to the software bottleneck effect.

The three architectures exhibited similar sensitivities to an increase in the inter-node delays. A reduction of approximately $72 \%$ in overall system throughput $(\mathrm{N}=24)$ of all architectures was observed when the inter-node delay was increased form 250 to $1000 \mathrm{~ms}$ 
with/without cloning.

Significant improvement in system throughput is attained as the number of cloned agents increases. An increase of $155 \%$ and $341 \%$ in the throughput was observed for the FORB and the $P-O R B$ respectively (at $N=24, D=250 \mathrm{~ms}$ ) when the cloning degree was increased form 1 to 8 . The P-ORB behaved worst with no cloning. However with 8 cloned agents, it ourperformed the H-ORB and the F-ORB. Similar patterns were observed for higher inter-node delays as well. 


\section{Chapter 6 The Adaptive ORB (A-ORB)}

\subsection{Motivation}

Each of the three architectures, the H-ORB, the F-ORB and the P-ORB has a special feature that makes it more attractive than the other in a specific situation. The marshalling/ unmarshalling cost is minimized in the H-ORB. Both, the F-ORB and the P-ORB result in a smaller number of message transfers in comparison with the $\mathrm{H}-\mathrm{ORB}$. The P-ORB also uses concurrency in server execution to improve performance. The analysis of the experimental results in Chapter 5 reveals that there is a trade-off between latency obtained at low load and throughput obtained at higher loads when no cloning is used. The reason behind that is the existence of software bottlenecks (details in Chapter 5 in Subsection 5.2.4.1). The analysis of the throughput curves of Figure 5-37 in Chapter 5 shows that while the number of clients is smaller than 4 , the F-ORB behaves better than the H-ORB. However, the H-ORB curve crosses over the F-ORB curve and exhibits a better performance when the number of clients goes beyond 4 . The experimental results also show that the degree of agent cloning has a direct effect on the position of the cross-over point. Therefore, designing an architecture that exhibits the best behavior of the H-ORB and the F-ORB under the whole range of load conditions warrants studying. This chapter covers a preliminary investigation of a new ORB architecture, the Adaptive ORB (A-ORB). The AORB combines the functionalities of both the H-ORB and the F-ORB and can switch dynamically from an $\mathrm{H}-\mathrm{ORB}$ mode to an F-ORB mode and vice versa depending on the 
load condition.

\subsection{Definition}

The $A-O R B$ is an architecture in which the agent, the $A$-agent (defined in the following section), based on the current load condition, switches its behavior to the ORB architecture that performs best under this load condition. In this introductory study, the a-agent, depending on the number of the waiting client requests, would behave as the default agent or the $f$-agent defined in Chapter 3. In other words, the A-ORB can dynamically switch from an $\mathrm{H}-\mathrm{ORB}$ mode to an F-ORB mode and vice versa. If the number of waiting client requests is less than a certain threshold, then the a-agent forwards the client request to the respective server which in turn performs the needed service (an f-agent behavior). However, once the number of waiting requests becomes equal to the threshold, the a-agent returns the handle of the sought server to the client, which in turn calls that server directly (a default agent behavior). In either case, the server replies back directly to the originating client. The name of the client or other identifying data, is embedded within the request so that the reply can be sent back directly without going through the $a$-agent. The high level interaction between the main components is shown in Figure 6-1. Since the Agent is a special process, its interface with the ORB core is presented through a different shade than that of the client and the server processes.

\subsubsection{The A-ORB Scenario}

The scenario below describes the behavior and roles of the components of the A-ORB client/server architecture. Figure 6-2 shows the execution sequence and interaction of the 


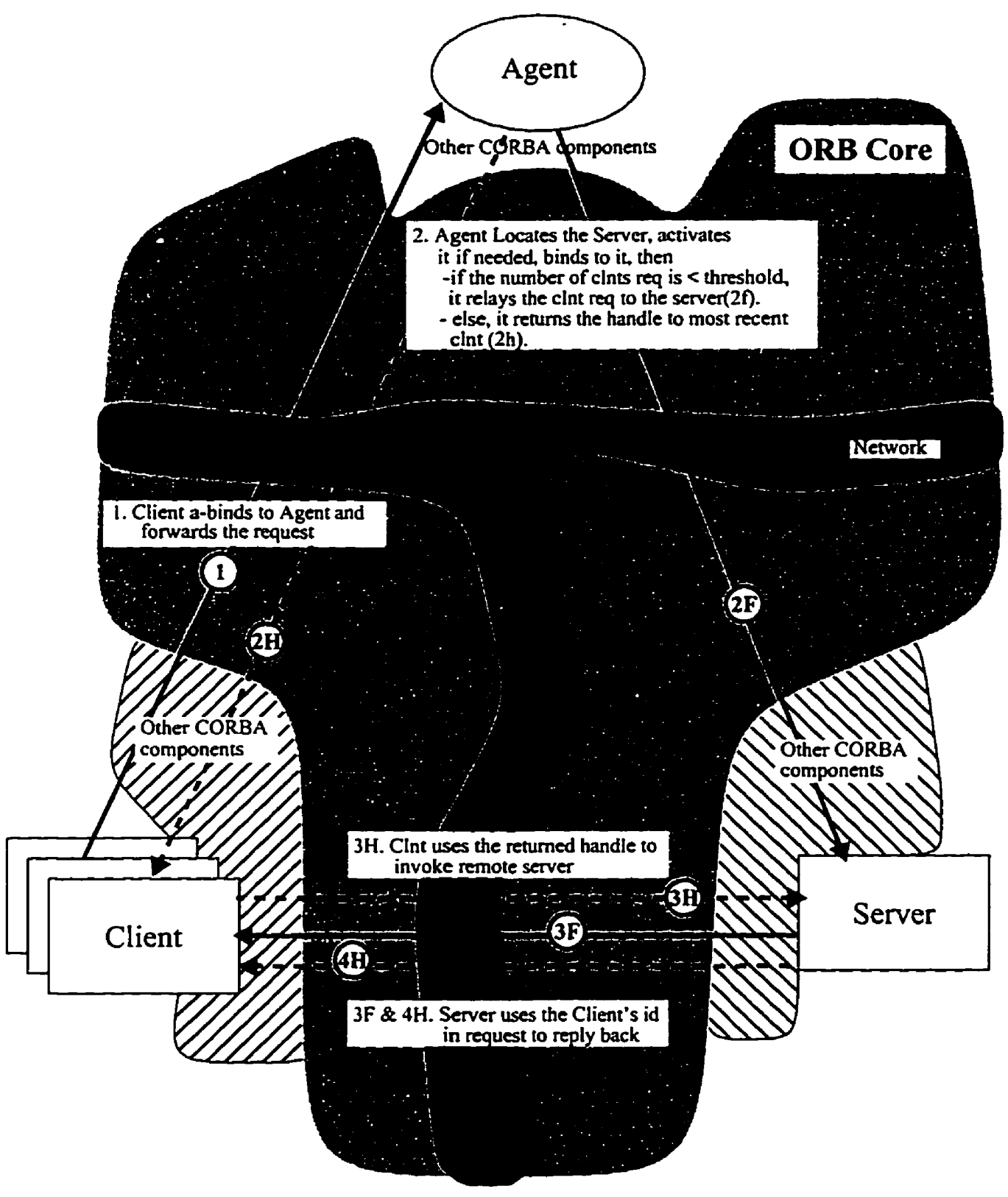

Figure 6-1: A high level interaction description of the A-ORB Architecture 
main components of this architecture. The description of the objects used in the execution scenario diagram is given in Chapter 4 in Figure 4-3.

\subsubsection{The Client}

1) The client rises and enters its initialization phase (details in Subsection 6.3.2.1) including the preparation of the needed arguments to be passed to the sought server.

2) The client, in accordance with the CORBA specifications, does not know nor care about the sought server location and must contact the ORB Agent in its domain to reach that server. Unlike the bind operation defined earlier in the previous architectures, the proposed binding operation, called hereinafter " $a$ bind", forwards the client request that includes the service needed and the types and values of the passed arguments to the Agent in the local domain of the client. Since the client is not always expecting a reply from the Agent, it is logical to implement the a-bind call as a "send oneway" call (as described in details in Chapter 4). Upon receiving the request on the Agent's side, the client becomes free to do any further activities.

3) Because the client is expecting a reply from the server (or any other process), it must register itself with the Agent to be accessible. After registration, the client can proceed to do some other tasks, including calling other remote services and receive replies or may choose to wait until the reply arrives from the remote server. In this case study, the client enters its main listening loop and waits for 
any messages.

4) When the client receives a message it terminates its listening mode.The client has the ability of knowing the type and reason for this call. In this case study the client faces the choices described below:

- The call is from the a-agent returning the handle of the desired server due to the high request load. In this case, the client uses this handle to invoke the remote server directly, via its stub, and then listens for the reply.

- The call is the server reply intercepted by the client skeleton, which in turn unmarshais the message parameters and passes them to the client. The client processes the final reply followed by some housekeeping activities (if need be), thereby ending its request cycle.

\subsubsection{The Agent's Dispatcher}

1) When activated, the dispatcher starts its initialization phase (details in Subsection 6.3.2.1). The dispatcher enters in main event loop and waits for incoming messages.

2) When an a-binding request arrives from a client, the dispatcher checks the number of enqueued client requests as explained next.

i) If the queue is empty and the agent is free, the request is forwarded to the agent. Otherwise the request is enqueued. The dispatcher goes into its main listening loop and waits for further requests.

ii) If the number of enqueued requests is fewer than the preset load threshold, 


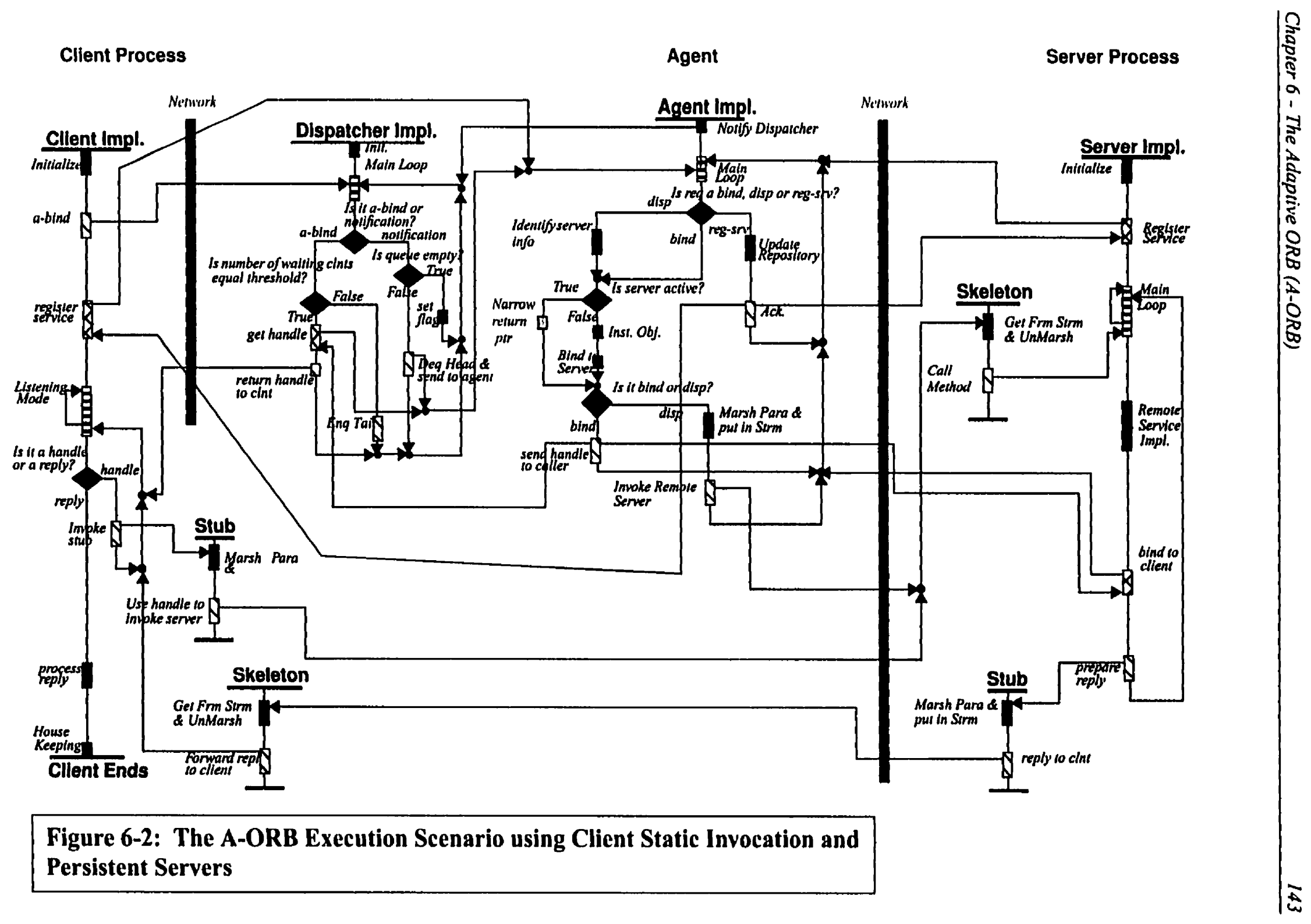


the dispatcher will enqueue the request and enter it main event loop.

iii) If the number of waiting requests is equal to the threshold, the dispatcher obtains the server's handle from the agent and returns the stringified handle of the server to the calling client, which in tum calls that server directly (identical to the default agent's behavior). As soon as the client gets the handle the dispatcher enters its main event loop.

3) When an agent notification arrives from the respective a-agent (the one on the same workstation), the dispatcher dequeues the request at the head of the queue and disparches it to the agent for further processing. However, if the queue is empty then the dispatcher sets an internal flag indicating that the agent is free. The dispatcher enters its main event loop.

\subsubsection{The Agent}

1) After getting activated, the agent notifies the dispatcher that it is free and enters its main event loop and waits for incoming messages.

2) When a Registration request arrives from a process, the agent uses the information in the request to update one or more of its repositories which are used later for locating and activating the respective processes. Then an acknowledgment message is sent back to the calling process.

3) If a client request is relayed from a dispatcher, then the agent extracts the information needed to identify the sought server from the message. The agent searches for the location and the status of the sought server in its repositories. 
If the server is active its reference is narrowed down to have it statically typed to the requested derived interface. Otherwise, if the server is inactive (nonpersistent as described in Subsection 4.3.2.3) then the address of its activation daemon, which performs the actual activation, is obtained. The agent marshals the request parameters and uses the obtained handle to send the message to the originally sought server using a send oneway call (identical to the f-agent's behavior). As soon as the server stub gets the message the agent is freed. It notifies the dispatcher enters its main event loop.

4) If a binding request arrives from an active process, then the agent looks for the location and the status of the sought server in its repositories. If the server is active, then its reference is narrowed down, to have it statically typed to the requested derived interface, and returned to the calling process. Otherwise, if the server is inactive (non-persistent) then the address of its activation daemon, which performs the actual activation, is returned to the calling process. The agent is freed and can enter its main event loop.

\subsubsection{The Server (Object Implementation)}

1) If the server is persistent (activated outside BOA), then it goes into its initialization phase. Afterwards, the server registers itself with the Agent, through its respective BOA, and waits for the Agent's acknowledgment. Once the acknowledgment is received, the server enters its main event loop: enters a listening mode, and waits for clients requests. 
2) If the server is non-persistent (not activated externally), then it must register itself with an activation daemon. This daemon activates the server (if not already active) upon the arrival of a client's request. This activation must be done in accordance with the Activation Policies stated in the H-ORB architecture in Subsection 4.3.2.3, when multiple objects or implementations exist. The server's designated BOA and daemon cooperate in delivering the client's request to it.

3) When a request arrives directly from the calling clients or being relayed from the Agent, the skeleton intercepts the request (the server can not identify the sender). It unmarshals the data and then calls the sought method in the object implementation.

4) Upon completing the request processing, a reply is sent (if the client is expecting one) using the send oneway mode described earlier. Based on the client name (or other identifying data) embedded in the request, the server must obtain the client's handle and use it to send the reply. Getting the handle can be achieved in one of the following approaches:

i) The handle can be obtained, stringified and embedded in the request by the Agent prior to relaying the request to the server. Hence the server would only need to unstringify the handle and use it.

ii) A smart server obtains the handle of the originating client from the Agent (only once during the life time of the experiment) and stores it locally for 
future invocations. (This is the approach used in the case study, details are in Subsection 6.3.2.1).

iii) A simple server calls the Agent each time a request arrives to get the handle.

5) The server uses the handle to send the reply back to the client through the server stub after marshalling the parameters using a send oneway call.

6) The server based on its activation policy, may go back into its main event loop or terminate.

\subsection{Case Study}

The experimental system is the same banking application described in Section 5.1. The following subsections describes the workload and the experimental environment.

\subsubsection{The Workload}

The construction of the workload is based on specifying the characteristics of the system as well as those of the applications executing on it that have a significant effect on the system performance. The main attributes that were investigated are as listed below, and a detailed description of the first four is presented in Subsection 5.1.1.

1) Message Size $(L)$ in Bytes.

2) Request Service Times $S_{A}$ and $S_{B}$ in msec.

3) Number of clients $(N)$.

4) Inter-Node Communication Delay (D) in msec. 
5) The Queue Length ( $Q L)$ in clients: This is the load threshold level set for the system. If the number of clients waiting at the a-agent dispatcher seen by an incoming request is equal to this threshold, the A-ORB agent returns the handle of the desired server to the calling client (switch to an H-ORB mode), otherwise the request is enqueued at the dispatcher (an F-ORB mode). In this introductory study of the A-ORB, the dispatcher's queue length is chosen to be the system load monitoring factor.

Table 23: Levels for the A-ORB Workload Factors

\begin{tabular}{|l|l|}
\hline \multicolumn{1}{|c|}{ Factors } & \multicolumn{1}{c|}{ Levels } \\
\hline $\mathrm{N}$ & $1, \ldots \ldots \ldots, 24$ \\
\hline $\mathrm{D}$ (msec) & 250,1000 \\
\hline $\mathrm{L}$ (bytes) & 150 \\
\hline $\mathrm{S}_{\mathrm{A}} / \mathrm{S}_{\mathrm{B}}$ (msec) & $10 / 15$ \\
\hline QL (clients) & $0,1,3$ \\
\hline
\end{tabular}

The different levels of the factors used in the experiments are listed in Table 23. The number and choice of the levels for each factor reflect a compromise between representativeness and an acceptable run time for the experiments.

\subsubsection{The Experimental Environment}

As described in all previous architectures, the system is comprised of a total of (14) Sun workstations ranging from Sparc-2 to Ultra workstations connected over a $10 \mathrm{Mbps}$ Ethernet LAN. A detailed description of the A-ORB architecture is presented along with the respective request path. 


\subsubsection{The Adaptive ORB A-ORB System}

The A-ORB system differs from the previous architectures in the sense that the a-agent may forward the reply to the sought server or return the handle to the requesting client depending on the load conditions (more details are given in the previous sections). The system consists of the following distributed Nodes:

a Client Nodes: The clients are distributed over a pool of available workstations as in the previous architectures. Details are in Subsection 5.1.2.1.

b Server Nodes: The server nodes are identical to those described in Subsection 5.1.2.1.

c Agent Node: This is a Sparc-20 workstation on which the default agent as well as the a-agent reside (no clients nor servers are installed on this machine).During each experiment, an a-agent is activated and set ready to receive and process any client request in cooperation with the default agent supplied by ORBeline. As explained later in the workload in the next section, the clients, the a-agent, and the servers need to obtain the handle of the sought process to forward the request to it. Having no access to the source code of the default agent has made impossible to modify it to include the functionalities of the a-agent in it in. Hence, the co-existence of both the default-agent and the a-agent on the same workstation is necessary to simulate their integration into a single complex agent and the default-agent presence is mandatory. Moreover, the a-agent process has its own dispatcher (a FIFO queueing process) on the same node working to monitor the client request load. The presence of this dispatcher is necessary since CORBA does not provide a means of accessing its internal buffers. However, when multiple a-agents are used, there will be a trade-off 
between having a single dispatcher for all agents or one dispatcher per agent. One dispatcher offers a better load balancing than multiple dispatchers, however larger queueing delays are faced by the client requests with one dispatcher than with multiple ones. Figure 6-3 illustrates the client Request Sequence Path for the A-ORB architecture.

d - The A-ORB Request Path: The request in this architecture follows a different path in comparison with the previous architectures described in Chapter 5. The a-agent, as described in the previous sections, is the process which, depending on the load conditions, relays the request to the sought server of either class or returns the handle of the server to the calling client. The path steps that are numbered in Figure 6-3 are described below. It should be noted that all of the path steps, excluding the initialization phase, use ORBeline's Pseudo Asynchronous (send oneway) calls to the called nodes. The initialization calls for the client, the servers, and the dispatcher are not shown in Figure 6-3.

- Client Initialization: In practice, the client should not ask the default agent for a handle to an a-agent (to its dispatcher in this case study) since both agents (the default and the a-agent) are integrated into a single process (complex agent) and can be contacted via a special ORB function call (the a-bind operation). Another issue is that the client should be able to unstringify the server handle returned by the dispatcher due to high load conditions. The unstringification approach was unsuccessful due to the same reasons stated in Subsection 5.1.2.2 in the F-ORB architecture. Hence, to make this architecture 


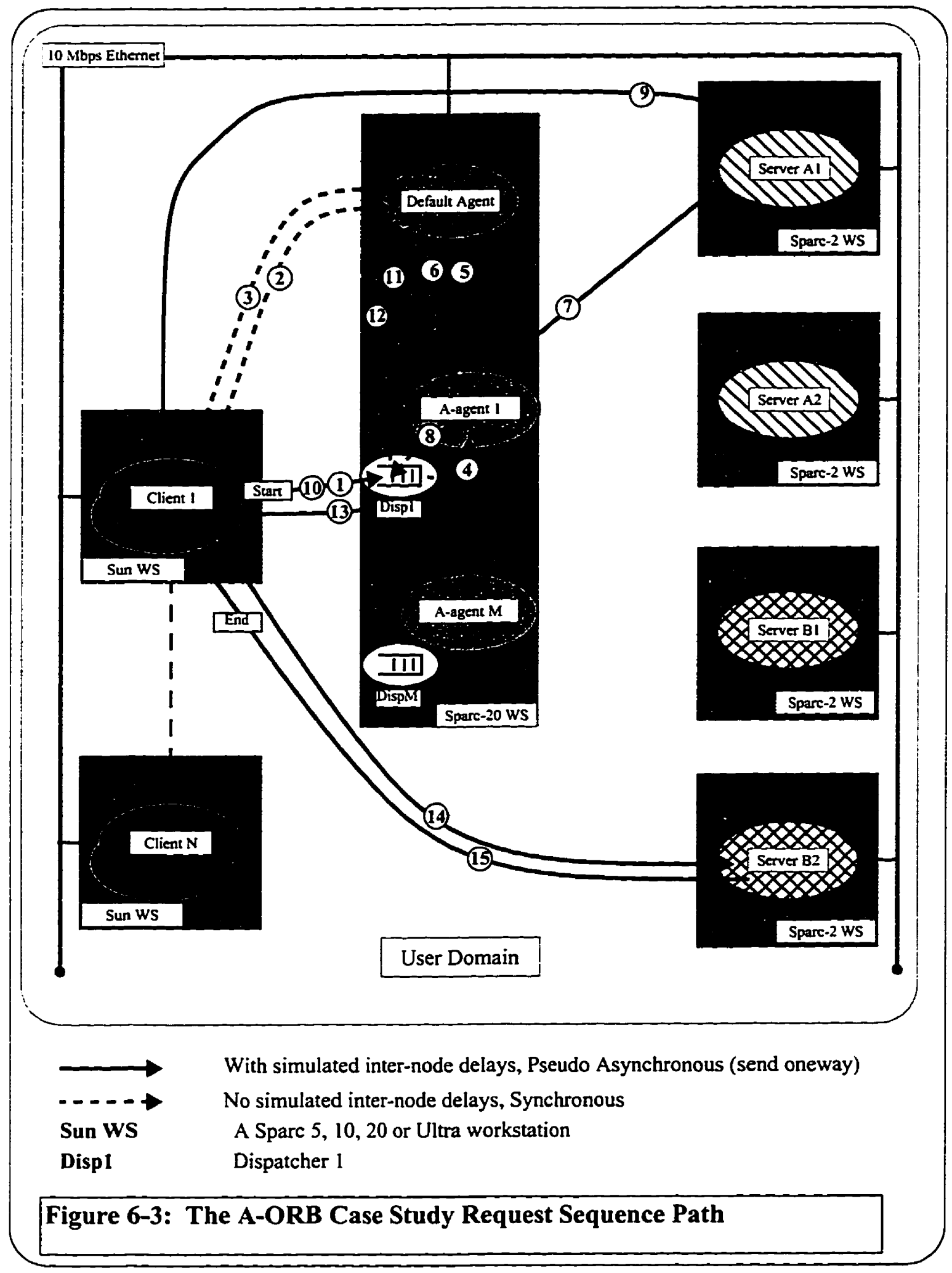


closer to the ideal A-ORB, the client obtains the handles for all the available $a$ agents' dispatchers and both class $A$ and $B$ servers and stores them locally.

- Server Initialization: Ideally, when a client request arrives at a server, its handle has already been stringified and embedded in the message by the agent. The server only needs to unstringify the handle and use it to return the reply. However, this approach, when implemented, was unsuccessful for medium to high system loads due to the problems raised up by ORBeline and Solaris. To simulate this ideal behavior, the server obtains the client's handle from the default agent while servicing the client's first request and stores it locally. For subsequent requests from the same client, the server searches its local data base to find out the respective client's handle and uses it to return the reply to the originating client.

- Dispatcher Initialization: The dispatcher, depending on the load conditions, may retum the stringified handle of the desired server to the calling client. However, due to the problems mentioned earlier with ORBeline and Solaris, an alternative approach was used to simulate this behavior. When the dispatcher wants to return a handle to the client, it obtains the server handle and releases it. A notification message is sent back to the client which in turns selects one of the stored handles randomly and use it to communicate with the server directly. Moreover, since the dispatcher, the agent and the default agent are ideally integrated into a single complex agent, during the initialization phase, the dispatcher obtains the handles of all the active clients and stores 
them locally for future reference.

- Step I: The client selects an a-agent, randomly, and uses its respective handle to send the request (thereby simulating the a-bind operation described earlier in this chapter). Let the $A$-agentI dispatcher be the process selected randomly by the client. The client forwards the request to this dispatcher using a send oneway call. The client sleeps before sending to the dispatcher to simulate the preset inter-node delay.

- Steps 2-3: The client registers itself with the default agent in order to be accessible to any other active process in the domain. No simulated inter-node delays are involved. This is done only once during the life time of the experiment.

- Step 4: The dispatcher checks the number of clients waiting in the queue to be serviced. In this case, it is assumed that this number is less than the preset threshold number. Hence, the dispatcher forwards the request to A-agentI using a send oneway call.

- Steps 5-6: $A$-agentl obtains a handle for a server of class $A$. Let $A I$ be the server, the handle of which is returned. This dynamic binding is needed to achieve load balancing and fault tolerance as well.

- Step 7: $A$-agent $I$ uses the handle obtained in (5-6) to relay the request to the $A I$ server using the send oneway mode. The a-agent sleeps before sending to $A I$ to simulate the preset inter-node delay.

- Step 8: As soon as the request reaches $A 1, A$-agent $l$ is freed and notifies its 
dispatcher that it is ready to service new requests. This is also done as a send oneway call.

- Step 9: In order for server $A l$ to return the reply (after processing the request) to the originating client, it must first have its handle. As discussed in the server initialization, $A l$ uses the respective handle, stored locally during the client's first request, to return the reply to the originating client using the same pseudo asynchronous mode. $A I$ sleeps prior to sending the reply to simulate the preset inter-node delay.

- Step 10: The client uses A-agentl dispatcher's handle to forward its request destined for a class $\boldsymbol{B}$ server using a pseudo asynchronous call to $A$-agent dispatcher. The client sleeping behavior is identical to that of stepl.

- Step 11-12: To demonstrate the dynamic change in the behavior of the AORB, it is assumed that the dispatcher, upon the arrival of the request, finds the number of client requests in the queue to be equal to the preset threshold value. The dispatcher binds to the sought server then releases the handle to simulate the ideal behavior of the A-ORB (details in dispatcher initialization).

- Step 13: The dispatcher does not sleep before replying to the calling client to simulate the behavior of the H-ORB. Once the client receives the reply, the dispatcher is freed and can enter its main event loop.

- Step 14: Upon the arrival of the reply from the dispatcher, the client sleeps to simulate the preset inter-node delay between the dispatcher and the client. The client then selects randomly a handle for a class $B$ server, stored locally, to 
simulate the ideal behavior (details in client initialization). Let B2 be the server, the handle of which is picked up. The client uses the handle to forward the request to $B 2$ using the send oneway call. The client sleeps before sending to simulate the preset inter-node delay.

- Step 15: In order for server $B 2$ to return the reply (after processing the request) to the originating client, it must first have its handle. As discussed in its initialization phase, $B 2$ uses the respective handle, stored locally during the client's first request, to return the reply using the same send oneway mode. $B 2$ sleeps prior to sending the reply to simulate the preset inter-node delay. The reception of the reply at the client side indicates the end of the client cycle.

\subsection{Experimental Results}

The A-ORB architecture was implemented. Sets of experiments were run to study the effect of the inter-node delays $\mathrm{D}$ on the relative performance of the H-ORB, F-ORB.V1 and the A-ORB architectures. The results of each experiment is presented as a set of graphs that capture the relationship between one of the performance measures of interest (plotted along the $y$ axis), and the number of clients $N$, (plotted along the $x$ axis) with all the other fixed parameters stated in the figure caption.

The number of client cycles was large enough to produce an interval that is less than $\pm 5 \%$ of the mean at a confidence level of $95 \%$ for the performance measure of interest. The impact of $\mathrm{D}$ and $\mathrm{QL}$ on the relative performance of the three architectures is covered in the next subsection. 


\subsubsection{The Effect of the Inter-Node Delay}

In this case study, only non-cloned architectures were considered, hence further studies should be performed to cover the other aspects of the system. The architecture main parameters are listed in Table 24 with the variable parameters in italic fonts. As in the previous case studies, the sender process sleeps before sending to simulate the inter-node delay.

Table 24: Parameters of the A-ORB Inter-Node Delay Experiments

\begin{tabular}{|l|l|}
\hline \multicolumn{1}{|c|}{ Main Parameters } & \multicolumn{1}{c|}{ Range of values } \\
\hline Number of Clients & $1,2,4,816,24$ \\
\hline Service Times & $\begin{array}{l}\text { Class A server }=10 \mathrm{~ms} \\
\text { Class B server }=15 \mathrm{~ms}\end{array}$ \\
\hline Inter-Node Delay & $10 \mathrm{~ms}, 250 \mathrm{~ms}, 1000 \mathrm{~ms}$ \\
\hline Message size & 150 Bytes \\
\hline $\begin{array}{c}\text { Degree of Cloning } \\
\text { H-ORB Arch } \\
\text { F-ORB Arch. } \\
\text { A-ORB Arch. }\end{array}$ & $\begin{array}{l}\text { Default Agent }=1 \\
\text { Default Agent }=1, \text { F-agents }=1 \\
\text { Default Agent }=1, \text { A-agents }=1\end{array}$ \\
\hline \begin{tabular}{c} 
Queue Length (A-ORB) \\
\hline
\end{tabular} & $0,1,3$ \\
\hline
\end{tabular}

In this case study, the effect of the queue threshold on system behavior is studied in two different scenarios:

Equidistant Nodes - The inter-node delay is the same between any two nodes in the system.

Non-Equidistant Nodes - In this case study it is assumed that the server nodes are closer to the agent node (the inter-node delay is set to $10 \mathrm{~ms}$ ), while the client nodes are far away from both (inter-node delay is set to $250 \mathrm{~ms}$ ). 


\section{a. Equidistant Nodes}

Inter-Node Delay: $250 \mathrm{~ms}$, Queue Length: 0,1,3-The influence of the inter-node delay as the number of clients increases on the mean client response time and the corresponding overall system throughput for the three architectures are shown in Figure 6-4 and Figure 65 respectively. Three A-ORB curves are presented in each figure to study the effect of the different thresholds $(0,1,3)$ used for the $A$-ORB queue length on system performance.

\section{Low Load Conditions:}

As shown in Figure 6-4 and Figure 6-5, while the number of clients, N, is 1 or 2 the A-ORB architectures for all the different values of QL are identical to the F-ORB. This is because with such a small number of clients, the queue length threshold is never exceeded and the A-ORB behaves like an F-ORB. As discussed in Subsection 5.2.6.1, the $\mathrm{H}-\mathrm{ORB}$ is worst due to its long path length. When $\mathrm{N}=4$, the $\mathrm{H}-\mathrm{ORB}$, the $\mathrm{F}-\mathrm{ORB}$ and the $\mathrm{A}-\mathrm{ORBs} / \mathrm{QL}=1,3$ all give comparable performance. This is due to the fact that the gain obtained by returning the handles to the clients is compensated by the extra time the a-agent is blocked waiting for a free server, as more clients are going to compete directly with the a-agent for the servers. As a consequence, the clients with enqueued requests at the dispatcher will face higher delays and have longer cycle times than those communicating directly with the servers. An examination of Figure 6-10 and Figure 6-11 shows that the process utilization of the a-agent is $100 \%$ which supports this observation. 


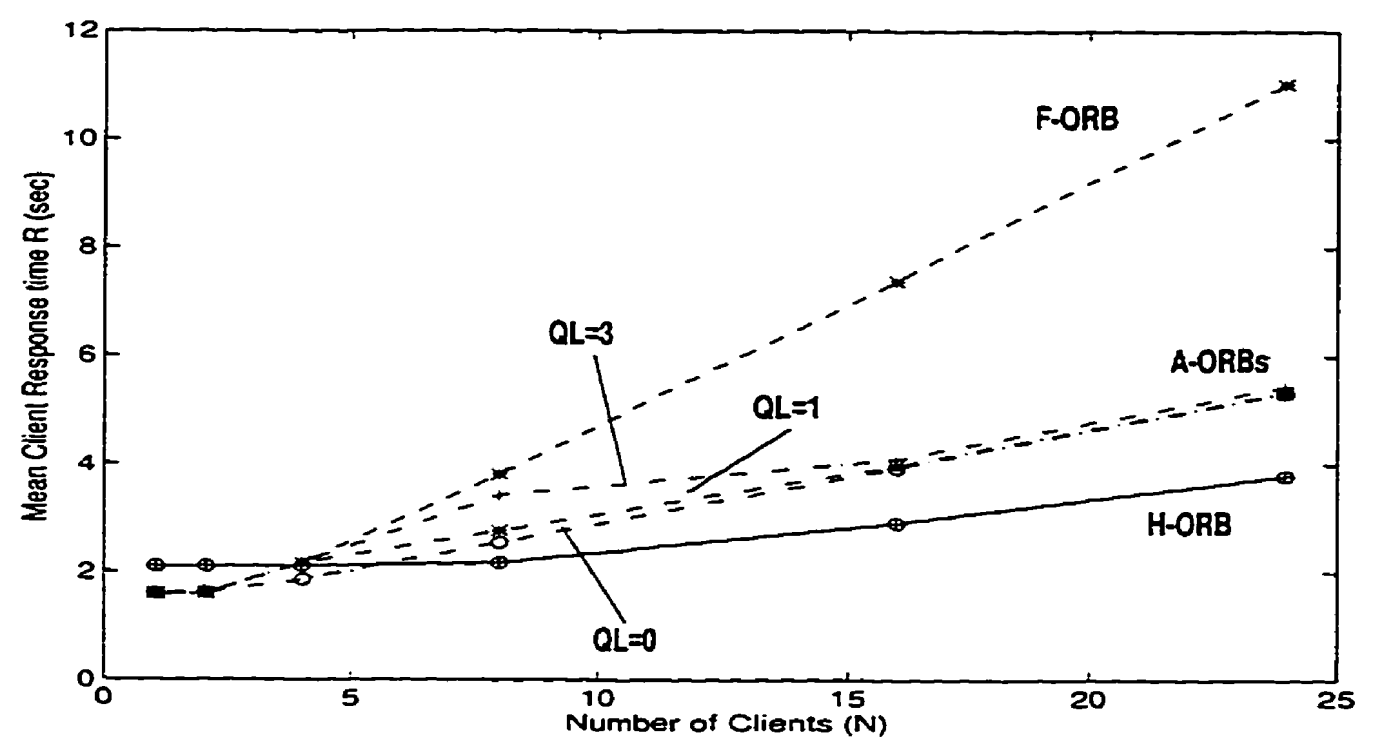

Figure 6-4: The Response Time of the H-ORB, the F-ORB and the A-ORB with Equidistant Nodes.

Degree of Cloning $=1$, Del=250 ms, $L=150$ Bytes, Server $A=10 \mathrm{~ms}$, Server $B=15 \mathrm{~ms}$. Queue Length $=0,1,3$.

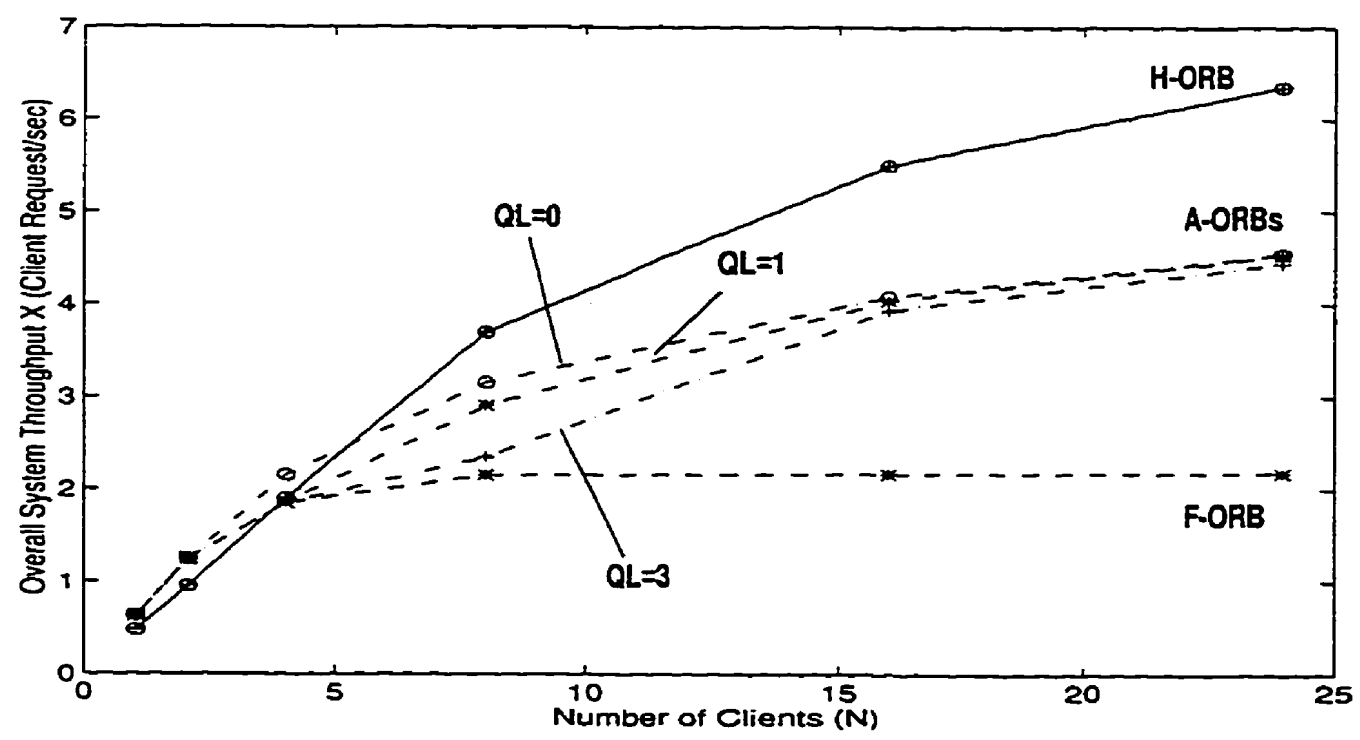

Figure 6-5: The Throughput of the H-ORB, the F-ORB and the A$O R B$, with Equidistant Nodes.

Degree of Cloning $=1$, Del $=250 \mathrm{~ms}, \mathrm{~L}=150$ Bytes, Server $A=10 \mathrm{~ms}$, Server $B=15 \mathrm{~ms}$. Queue Length $=0,1,3$. 


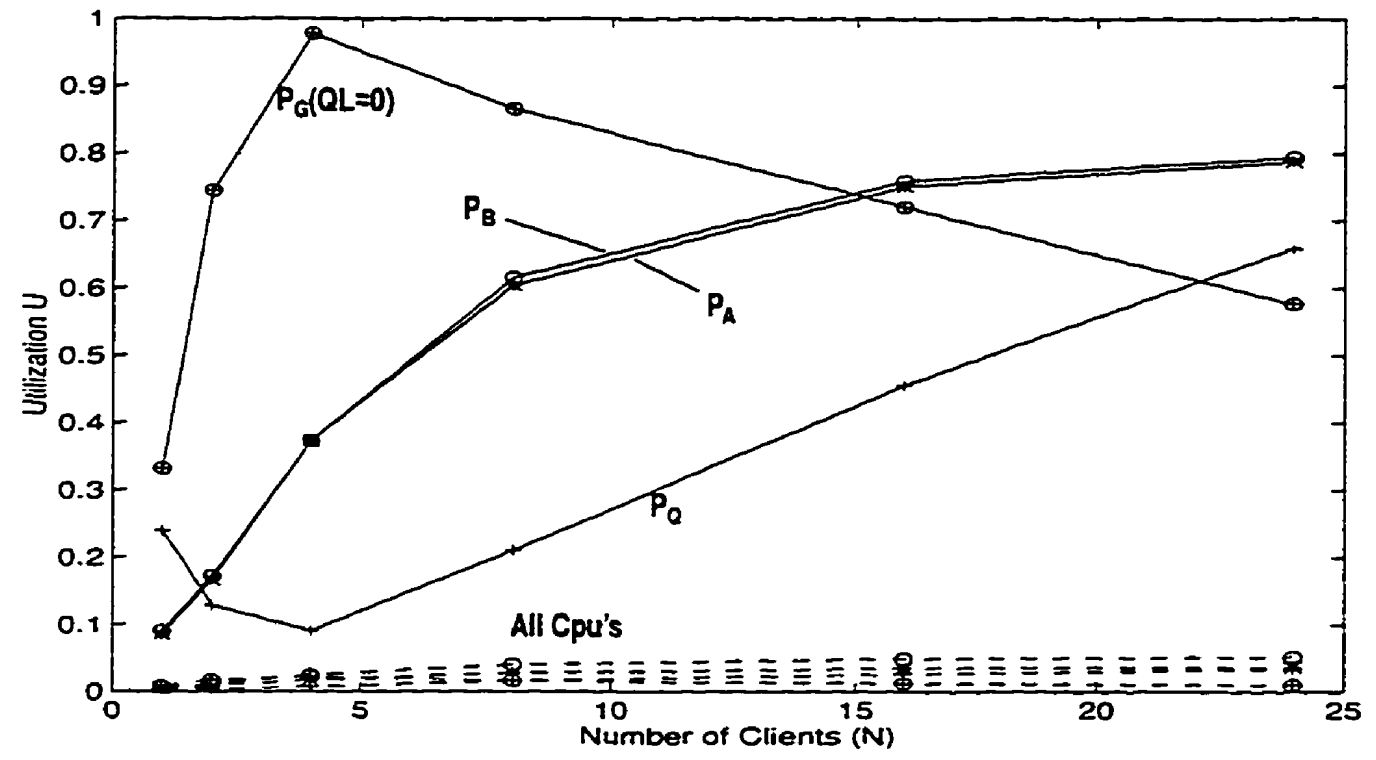

Figure 6-6: The Process and Cpu Utilizations of the A-ORB (QL=0)

$P_{A}=$ Process Util of Server $A, C P U_{A}=C p u$ Util of server $A, P_{G}=$ Process Util of a-agent, $P_{Q}=$ Process Util of Dispatcher, Degree of Cloning $=1$, Del=250 ms, $L=150$ Bytes, Server $A=10 \mathrm{~ms}$, Server $B=15 \mathrm{~ms}$, Queue Length $=0$

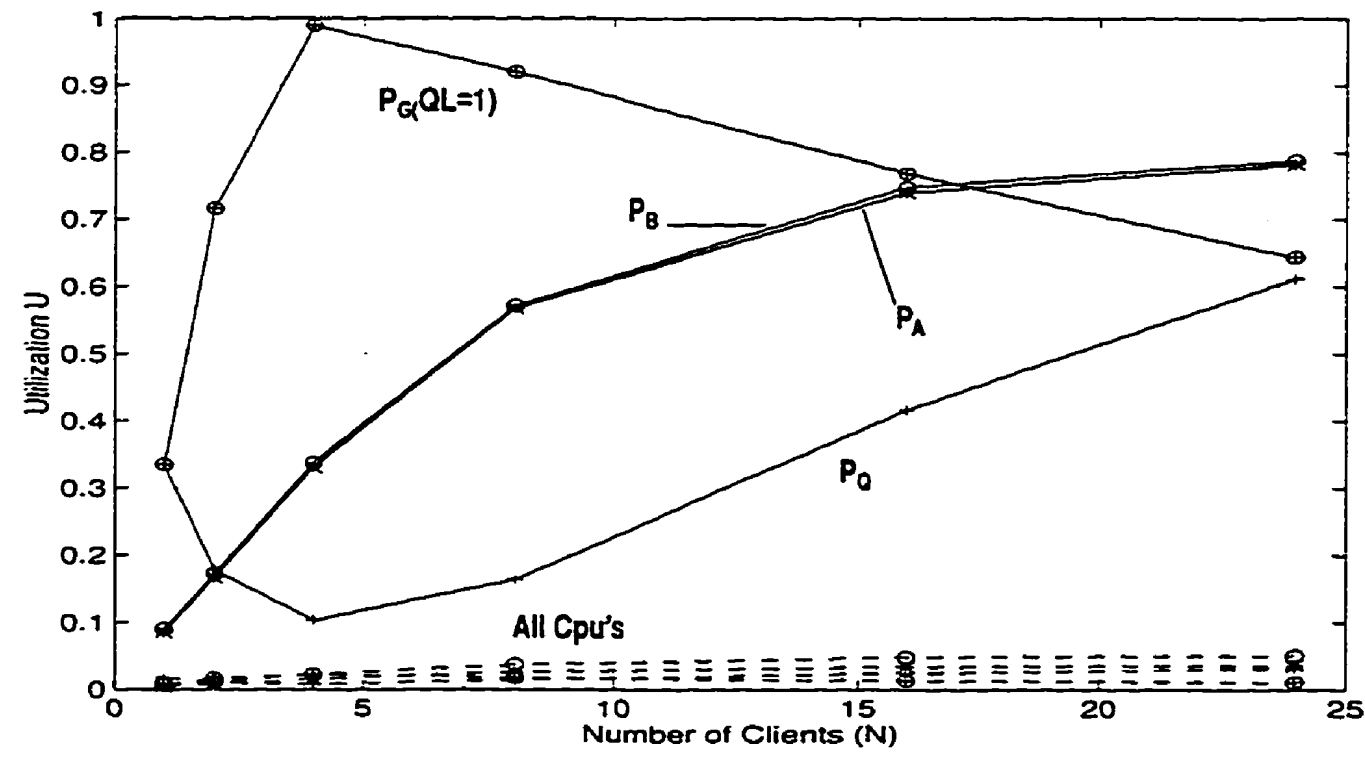

Figure 6-7: The Process and Cpu Utilizations of the $A-O R B(Q L=1)$

$P_{A}=$ Process Util of Server $A, C P U_{A}=C p u$ Util of server $A, P_{G}=$ Process Util of a-agent, $P_{Q}=$ Process Util of Disparcher, Degree of Cloning $=1$, Del=250 ms, $L=150$ Bytes, Server $A=10 \mathrm{~ms}$, Server $B=15 \mathrm{~ms}$, Queue Length $=1$. 


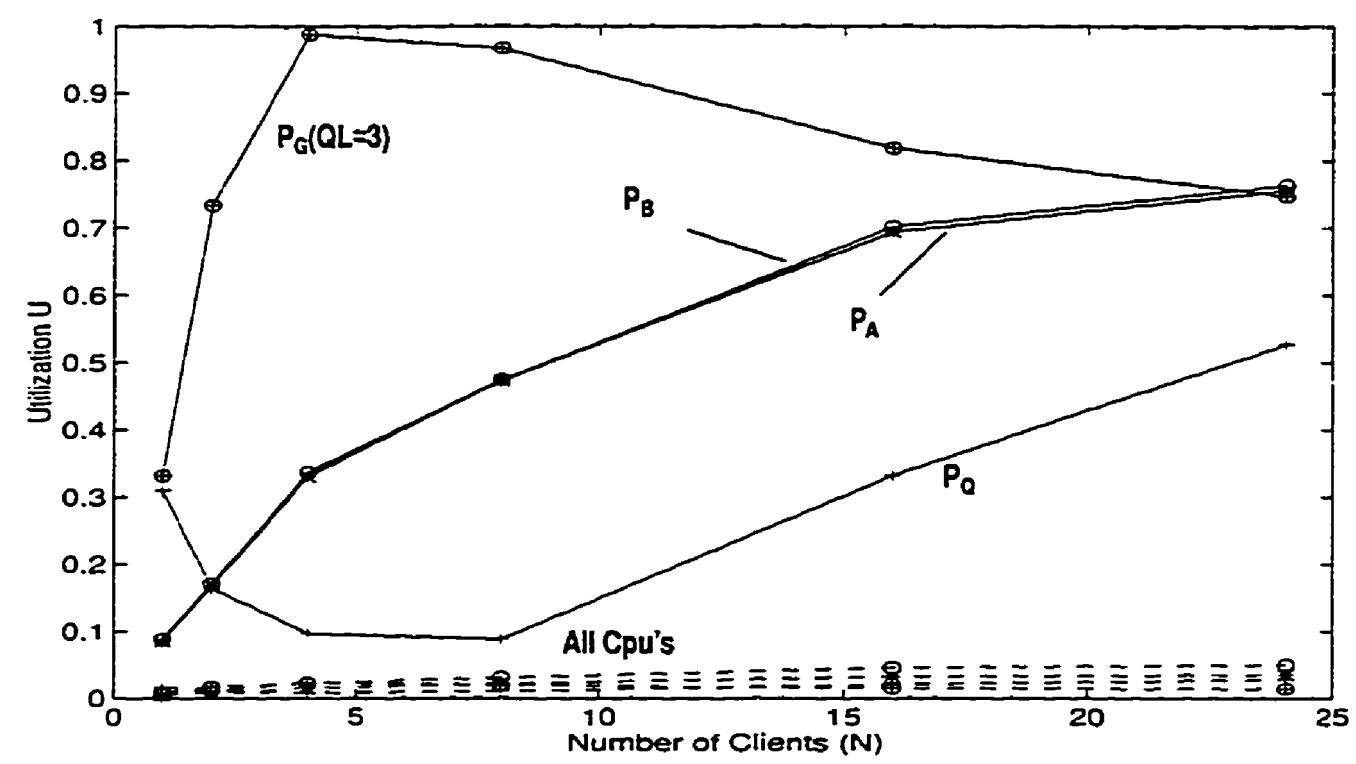

Figure 6-8: The Process and Cpu Utilizations of the A-ORB (QL=3) $P_{A}=$ Process Util of Server $A, C P U_{A}=C$ pu Util of server $A, P_{G}=$ Process Util of a-agent. $P_{Q}=$ Process Util of Dispatcher, Degree of Cloning $=1$, Del $=250 \mathrm{~ms}, \mathrm{~L}=150$ Bytes, Server $A=10 \mathrm{~ms}$, Server $B=15 \mathrm{~ms}$, Queue Length $=3$.

However, the $A-O R B / Q L=0$ exhibits the best performance surpassing even the $H$ ORB. The reason behind that is the gain obtained by returning the handle to any client calling the a-agent if the a-agent is currently busy with another request. As a result, no requests are enqueued and the extra delays are avoided. This is manifested by the relatively lower utilization of the a-agent at $N=4$ (shown in Figure 6-6) compared with the other $\mathrm{A}-\mathrm{ORB} / \mathrm{QL}=1,3$ architectures.

\section{Medium to High Load Conditions:}

As soon as $\mathrm{N}$ goes beyond 5 , the $\mathrm{H}-\mathrm{ORB}$ takes the lead as the best architecture while the F-ORB becomes worst. Two distinct regions are observed for all A-ORB architectures: 
In the first region, when $N$ is between 4 and 16, and especially for $N=8$ the $A-O R B /$ $\mathrm{QL}=0$ is best due to the maximum reduction of queueing delays at the a-agent. As the QL level becomes larger, more clients are forced to remain queued behind the a-agent. Consequently, the throughput is reduced and their respective curves are pulled down towards the F-ORB curve. As $\mathrm{N}$ approaches 16 , the A-ORB architectures for all values of QL exhibit close performance, since the utilization of the a-agent and the two servers becomes closer to each other. Hence the system performance will be affected by both of them.

The second region covers the range of $\mathrm{N}$ when it is between 16 and 24 . It is noticed that the A-ORB architecture maintains very close performance with all the three values of QL.

Inter-Node Delay: 1000 ms, Queue Length: 0,1,3 - Identical behavioral patterns were also observed as shown in Appendix D from Figure D-1 to Figure D-5.

\section{b - Non-Equidistant Nodes}

Inter-Node Delay: $250 / 10 \mathrm{~ms}$, Queue Length: 0,1,3 - The mean client response time and the corresponding overall system throughput are shown in Figure 6-9 and Figure 6-10 respectively. The two main observations are First, is the behavior of the F-ORB.VI is significantly improved and surpassed the H-ORB as long as the number of clients are lower than 20 due to the reduced waiting time of the $\mathrm{f}$-agent. The second is the A-ORB behavior. In contrast to the equidistant node case study, the system throughput increases as the queue threshold goes form 0 to 1 to 3 when $\mathrm{N}$ is less than 8 . This is due to the fact that returning 


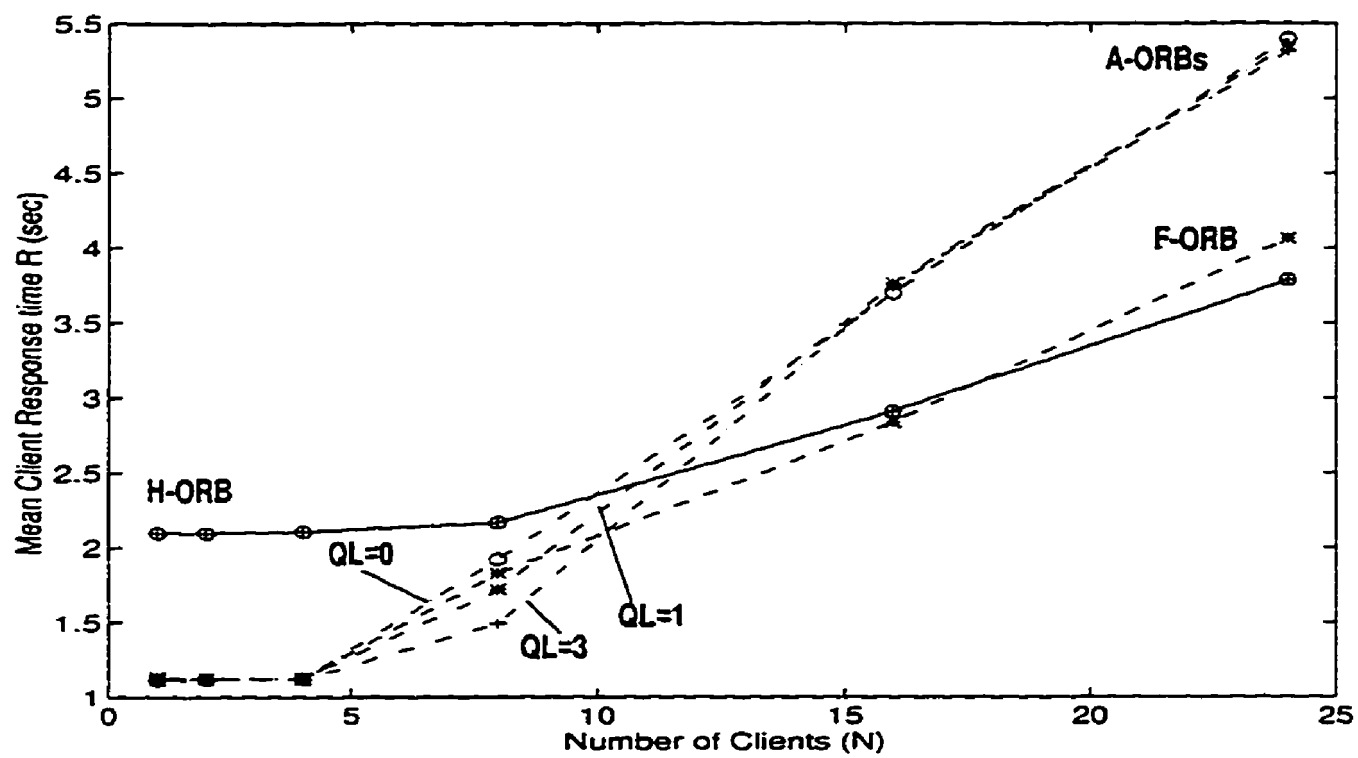

Figure 6-9: The Response Time of the H-ORB, the F-ORB and the A-ORB with Non Equidistant Nodes.

Degree of Cloning $=1$, Client $->$ Agent $/$ Server del $=250 \mathrm{~ms}$, Agent $\rightarrow$ Server del $=10 \mathrm{~ms}, \mathrm{~L}=150$ Bytes, Server $A=10 \mathrm{~ms}$, Server $B=15 \mathrm{~ms}$, Queue Length $=0,1,3$.

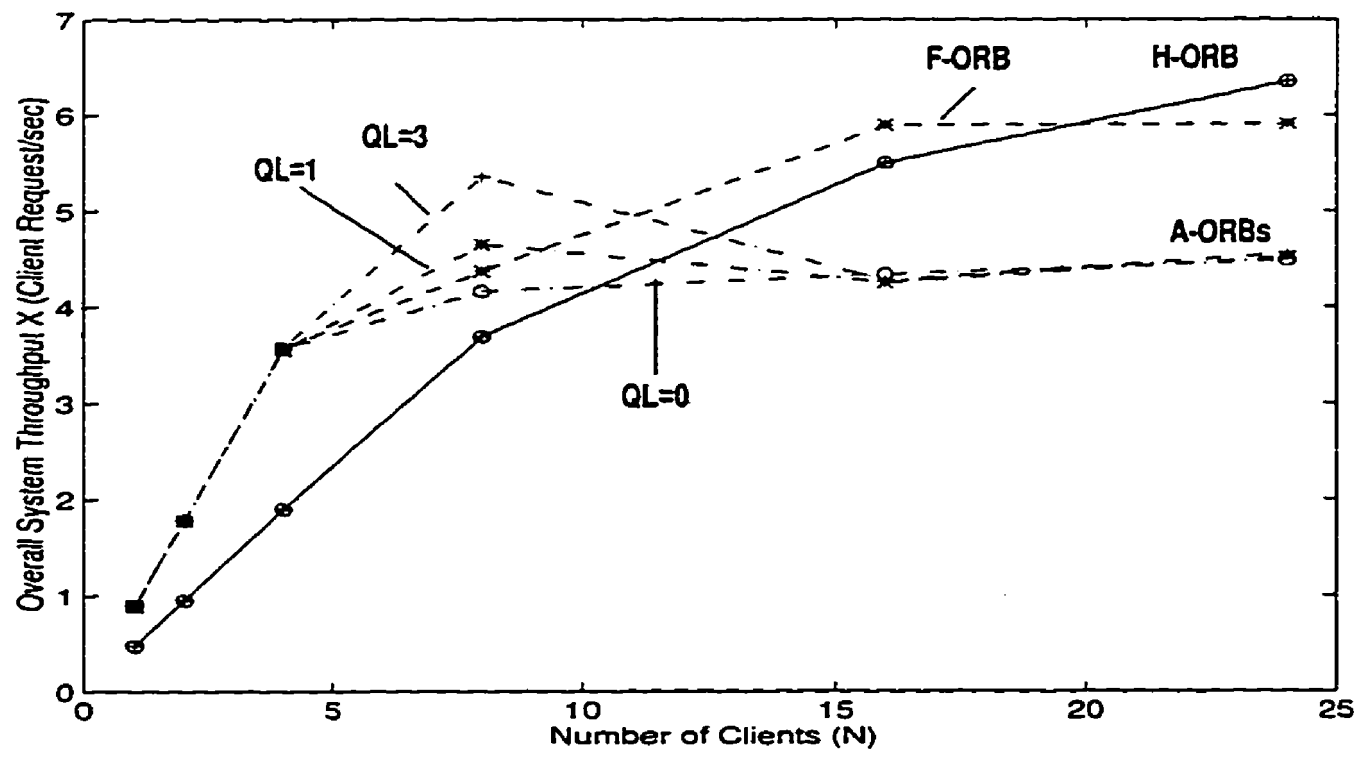

Figure 6-10: The Throughput of the H-ORB, the F-ORB and the AORB with Non Equidistant Nodes.

Degree of Cloning $=1$, Client $->$ Agent Server del $=250 \mathrm{~ms}$, Agent $\rightarrow$ Server del $=10 \mathrm{~ms}, \mathrm{~L}=150$ Bytes, Server $A=10 \mathrm{~ms}$, Server $B=15 \mathrm{~ms}$, Queue Length $=0,1,3$. 


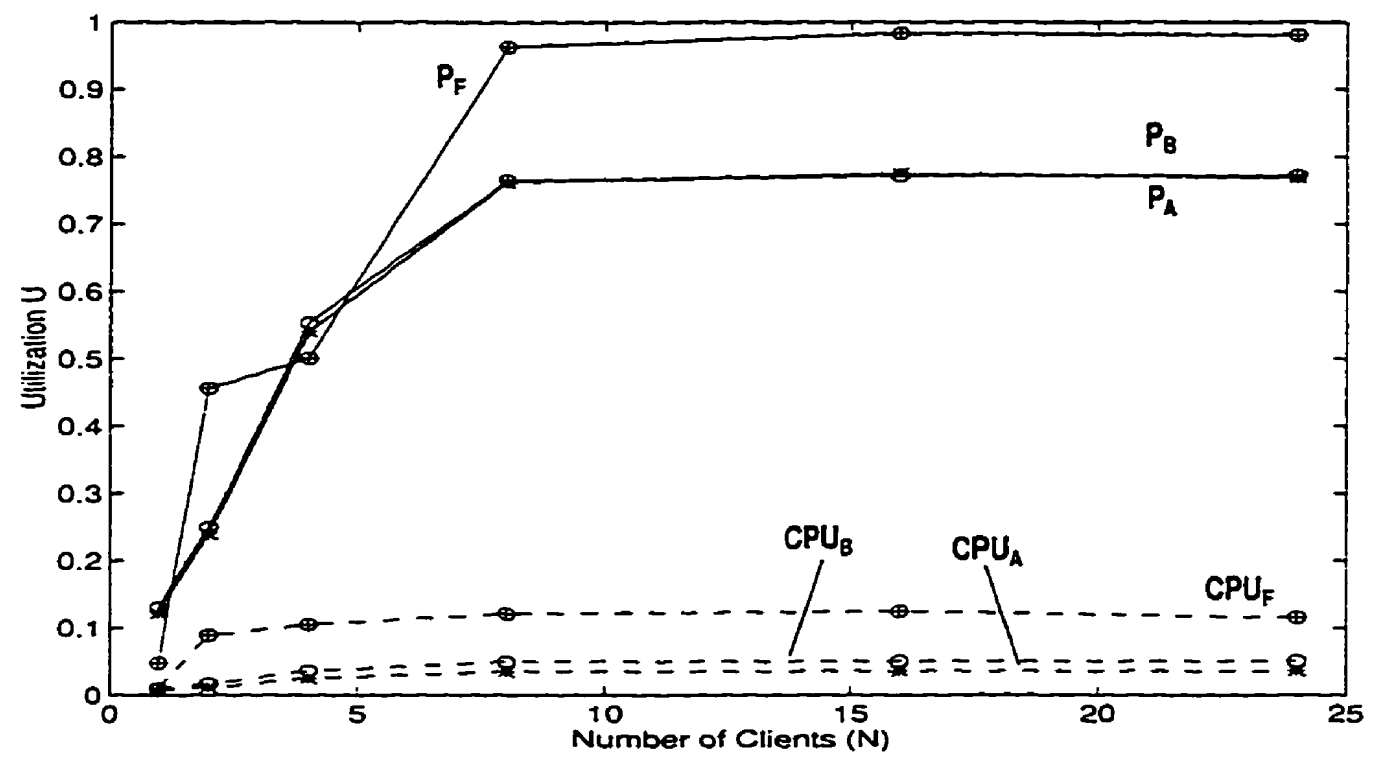

Figure 6-11: The Process and Cpu Utilizations of the F-ORB with Non Equidistant Nodes.

$P_{A}=$ Process Util of Server A. $C P U_{A}=$ Cpu Util of server $A_{,} P_{F}=$ Process Util of $f$-agent Degree of Cloning $=1$, Client-> Agent/Server del $=250 \mathrm{~ms}$. Agent $\rightarrow$ Server del $=10 \mathrm{~ms}, \mathrm{~L}=150$ Bytes, Server $A=10 \mathrm{~ms}$, Server $B=15 \mathrm{~ms}$.

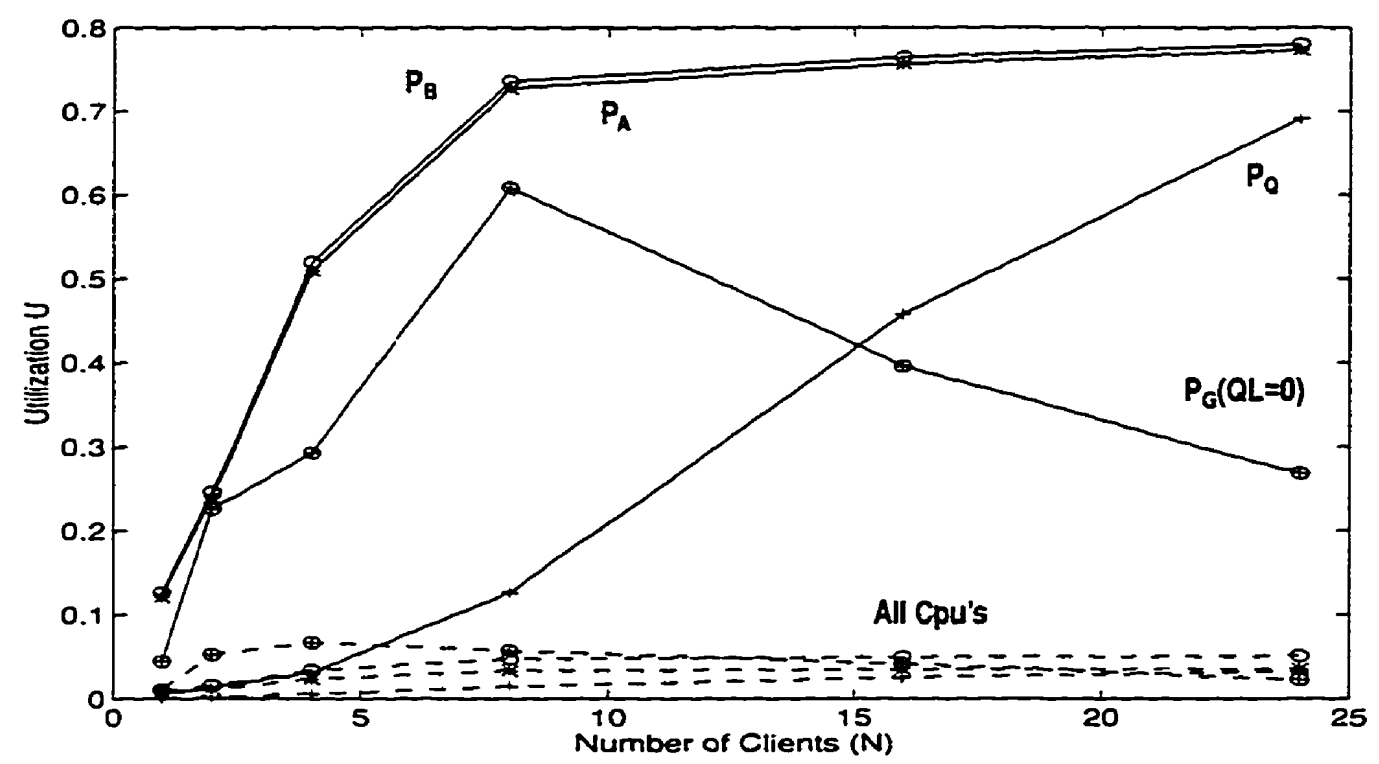

Figure 6-12: The Process and Cpu Utilizations of the A-ORB with Non Equidistant Nodes $(\mathrm{QL}=\mathbf{0})$

$P_{A}=$ Process Util of Server $A, C P U_{A}=C p u$ Util of server $A, P_{G}=$ Process Util of a-agent. $P_{Q}=$ Process Util of Dispatcher, Degree of Cloning $=1$, Client $\rightarrow$ Agent/Server del $=250 \mathrm{~ms}$,

Agent $\rightarrow$ Server del $=10 \mathrm{~ms}, L=150$ Bytes, Server $A=10 \mathrm{~ms}$, Server $B=15 \mathrm{~ms}$, Queue Length $=0$. 


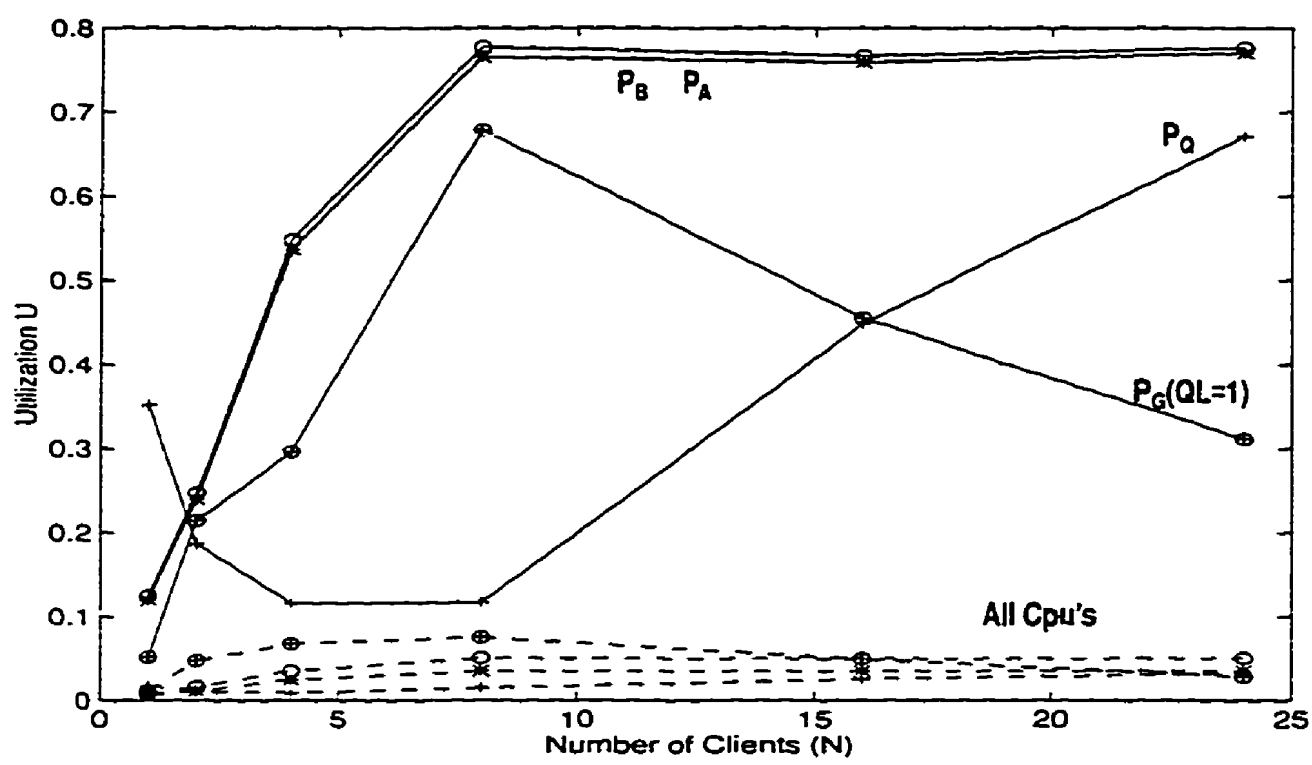

Figure 6-13: The Process and Cpu Utilizations of the A-ORB with Non Equidistant Nodes (QL $=1$ )

$P_{A}=$ Process Util of Server $A, C P U_{A}=C p u$ Util of server A,P $P_{G}=$ Process Util of a-agent, $\mathrm{P}_{\mathrm{Q}}=$ Process Util of Dispatcher, Degree of Cloning $=1$, Client- $>$ Agent $/$ Server del $=250 \mathrm{~ms}$, Agent $\rightarrow$ Server del $=10 \mathrm{~ms}, L=150$ Bytes, Server $A=10 \mathrm{~ms}$, Server $B=15 \mathrm{~ms}$, Queue Length $=1$.

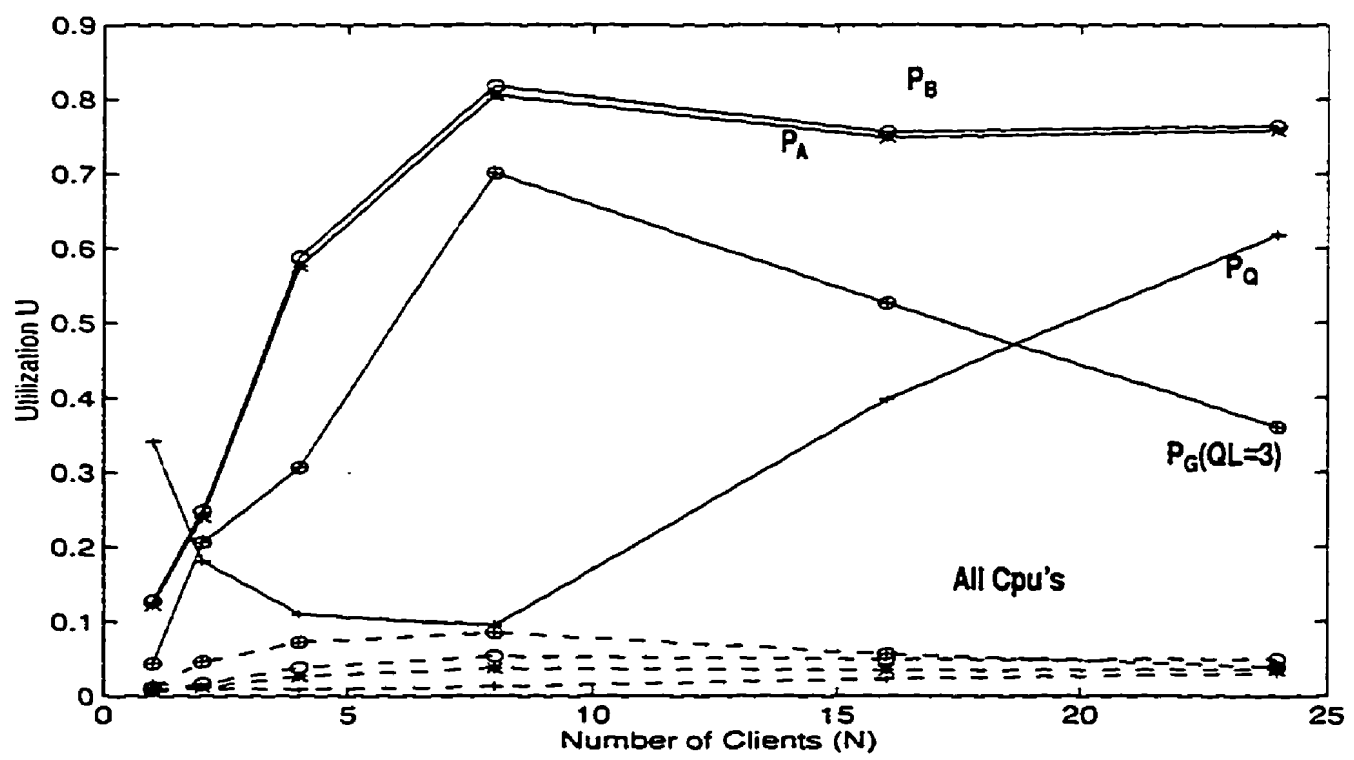

Figure 6-14: The Process and Cpu Utilizations of the A-ORB with Non Equidistant Nodes (QL=3)

$P_{A}=$ Process Util of Server $A, C P U_{A}=C p u$ Util of server $A, P_{G}=$ Process Util of a-agent, $P_{Q}=$ Process Util of Dispatcher, Degree of Cloning $=1$. Client $\rightarrow$ Agent $/$ Server del $=250 \mathrm{~ms}$, Agent $\rightarrow$ Server del $=10 \mathrm{~ms}, L=150$ Bytes, Server $A=10 \mathrm{~ms}$, Server $B=15 \mathrm{~ms}$, Queue Length $=3$ 
the handle would cause the request to go through a longer path than that through the aagent. As $\mathrm{N}$ increases even further, the performance of $\mathrm{A}-\mathrm{ORB}$ architecture for all different thresholds converge to the same value due to the saturation of both servers. Therefore, the queued requests will face longer delays. The utilization graphs in Figure 6-11 to Figure 614 can be consulted for more details.

\subsubsection{Discussion}

It is evident from the previous experiments that the queue length threshold have a significant effect on the system performance especially at medium load. The current set of experiments has revealed that the threshold value to be used is determined by the relative delay among the various nodes of the system.

However, more experiments are needed to study the effect of other factors including the cloning of the agent, the message size and the server demands. Further studies should be done to investigate the possibility of integrating the P-ORB functionalities into the A-ORB. 


\section{Chapter 7 Conclusions}

\subsection{Summary}

The main focus of the thesis was the design and implementation of three different CORBA-based client-server architectures, the H-ORB, the F-ORB, and the P-ORB using ORBeline, and analyzing their performance. In order to identify the best architecture, the relative performance of those three architectures was investigated under different workload and environment conditions. Description of the experiments as well as the interpretation of the results are presented in chapter 5 in section 5.3.

One of the contributions of the thesis is the introduction of a forth architecture the AORB. Based on the insights gained from analyzing the behavior of the H-ORB and the F$\mathrm{ORB}$, the A-ORB was designed to combine the functionalities of both of the H-ORB and the F-ORB in addition to the ability to dynamically switch its behavior from an H-ORB mode to an F-ORB mode and vice versa in response to a change in system load. A preliminary research has been done on the relative performance of the A-ORB and its parent architectures under a selected set of workload and environment conditions. The interpretation of the results are given in chapter 6 in section 6.4 .

\subsection{Gained Insights}

A number of insights have been gained from the experimental analysis and can be used as guidelines for system designers and users. These insights are based on the observations 
of the behavior of CORBA-based systems as captured by the ORBeline product and include the following.

a None of the architectures exhibited the existence of a hardware bottleneck. However, software bottlenecks have been identified in each of the F-ORB, P-ORB and the $A-O R B$.

b - Agent cloning lead to a significant improvement in system performance in almost all cases. Hence it is a strongly recommended approach.

c. The message size and the inter-node delays have a strong impact on system performance. Hence, careful consideration should be made with respect to these two factors when selecting the appropriate ORB architectures.

d - Latency-Scalability Trade-off: There seems to be a trade-off between latency obtained at low load and throughput obtained at higher loads when no cloning is used. The architecture that exhibits best latency at low load may rank lower when scalability at high load is considered. Hence, a combined latency/scalability profile of the architecture must be taken into consideration.

e - Sequential versus Concurrent Invocation: The P-ORB uses concurrency in server invocations to improve performance. As a result, its performance can be significantly higher than that of the F-ORB or the H-ORB. Therefore, if the client's composite request consists of a number of independent constituent requests, the P-ORB can be a very beneficial choice in many cases.

f - Threshold factor: The dynamic behavior of the A-ORB is controlled by a preset value 
of a specific system parameter. In this introductory study of the A-ORB, the impact of the preset threshold value on system performance was found to be strongly related to the given environment conditions.

\subsection{Derived Guidelines}

The overall analysis of the results indicates that there is no ideal ORB architecture that performs best under all conditions. However, knowing the workload requirements for the application, the suitable architecture can be identified for a given environment. Based on the insights stated in the previous section, the following guidelines are recommended:

- Communication Bound (large message size) Applications - Without agent cloning, the $\mathrm{P}-\mathrm{ORB}$ is best for lightly loaded systems, while the H-ORB ranks first for medium to high load levels. If cloning is applicable, then high degree of cloning is recommended and the P-ORB seems to be the best choice for all load level.

- Computation (server demand) Bound Applications - For large independent server demands, the P-ORB seems to be the best for all load levels of cloned/non-cloned systems.

- Environment Bound (large inter-node delay) Applications - For non-cloned systems, the P-ORB is best at low load levels, while the H-ORB is best for medium to high load levels. With high degree of cloning, the P-ORB is best over all load levels.

\subsection{Thesis Contributions}

The main contributions of this thesis are:

- The design and implementation of three different ORB architectures based on a 
commercially available product.

- An investigation of the relative performance of the three client-server architectures for which, to the best of our knowledge, no previous results existed.

- The identification of agent cloning as an important approach in CORBA-based clientserver architectures to improve performance.

- Insights and guidelines to be used by system designers and users to assist in selecting the best architectures.

- The proposal of a new ORB architecture, the A-ORB and the preliminary investigation of its performance.

A portion of the research presented in this thesis has been summarized in a paper entitled "Performance Comparison of Architectures for Client-Server Interactions in CORBA", has been submitted for publication.

\subsection{Future Research}

This research leads to a number of future research areas some of which are presented:

- More investigation is needed on the performance of the P-ORB architecture in cases where the client composite request contains multiple levels of dependent/independent constituent requests.

- Choosing an appropriate threshold for the A-ORB architecture requires further investigation of the system performance under different workload and environment conditions. Integrating more features into the agent of the A-ORB such as the PORB's decomposing ability warrants studying. Along the same avenue of research is 
agent cloning. Since agent cloning has proved itself to be very beneficial, studying the performance of cloned A-ORB architectures is important.

- Agent cloning has been implemented using multiple processes. To reduce the overhead of context switching, multithreading of the agent calls for investigation. Three different threading policies: thread per request, thread per client, and thread pool are suggested in [SCH-96.2]. Performance of these policies in the context of pattern-based design are discussed in [SOM-97]. Investigating these policies in the context of CORBA agents is important.

- Scheduling in client-server systems has an important impact on system performance. Therefore, scheduling of requests at the CORBA agent is another important area of research. Scheduling policies can be based on several factors such as request priority, path length and server demands.

- All the research in this thesis was performed using static invocation of persistent servers. More research needs to be done to examine the performance of all architectures using dynamic invocation of transient/persistent servers.

- All CORBA implementation architectures use stationary agents. However, in many cases, the network traffic is confined between a set of client and server nodes. In cases where the agent node is located far away from all other nodes, the concept of a mobile agent comes into the picture. A mobile agent is the agent which, based on the network traffic state and the relative location of its node, can migrate into a closer node to improve the system performance. Studying the performance of such mobile agents is worth investigation. 


\section{References}

[ADL-95] R. Adler, "Distributed Coordination Models for Client/Server Computing", IEEE Computer, pp 14-22, April 1995.

[BAN-84] J. Banks and J.S. Carson,II, "Discrete Event System Simulation". Prentice-Hall Inc., Englewood Cliffs, N.J. 07632, 1984.

[CHA-96] D. Chappell, "CORBA and DCE: What is the Story?", Chappell \& Associates, Minneapolis, Minnesota.

[ELG-94] T. Elgillani, A. Hubbard, C. Schramm, and F. Sheikh, "DECALS, Distributed Experiment Control and Logging System, User's Guide and Reference Manual", Real-time and Distributed Systems Group, Carleton University, 1125 Colonel Bye Drive, Ottawa, Ontario, Canada K1S 5B6, August 1994..

[FON-89] M.J. Fontenot, "Software Congestion, Mobile Servers, and the Hyperbolic Model", IEEE Transactions on Software Engineering, Vol. 15, 1989, pp. 947-962.

[LAV-95] R.G. Lavender and D.C. Schmidt, "Active Object: an Object Behavioral Pattern for Concurrent Programming", Proceedings of the $2^{\text {nd }}$ Annual Conference on the Pattern Languages of Programs, (Monticello, IIl), pp 17, Sept. 1995.

[LOC-94] H.W. Lockhart, Jr., "OSF DCE Guide to Developing Distributed Applications", McGraw Hill, Inc., 1121 Avenue of the Americas, New York, N.Y. 10020. 
[MAN-95] Solaris 2.5 On-line Reference Manuals, Sun Microsystems, Feb. 1, 1995

[NEI-95] J.E. Neilson, C.M. Woodside, D.C. Petriu, S. Majumdar, "Software Bottlenecking in Client-Server Systems and Rendezvous Networks", IEEE Transactions on Software Engineering, Vol. 21, No. 9, September 1995, pp 776-782.

[OMG-91] Object Management Group, "The Common Object Request Broker: Architecture and Specification, OMG TC Document Number 91.12.1, Rev. 1.1ed., Dec. 6, 1991.

[OMG-93] Object Management Group, "The Common Object Request Broker: Architecture and Specification", 1.2 ed., 1993.

[OMG-95] Object Management Group, "The Common Object Request Broker: Architecture and Specification", 2.0 ed., July 1995.

[ORB-94.1] PostModern Computing Technologies Inc., "ORBeline User's Guide" Mountain View, CA 94043, 1994.

[ORB-94.2] PostModern Computing Technologies Inc., "ORBeline Reference Guide”, Mountain View, CA 94043, 1994.

[OTT-96] R. Otte, P. Patrick, and M. Roy, "Understanding CORBA (The Common Object Request Broker Architecture)", Prentice Hall PTR, A Simon and Schuster Company, Upper Saddle River, New Jersy 07458, 1996.

[PRA-95] S. Prata, "C++ Primer Plus", Waite Group Press, Corte Madera, CA $94925,1995$.

[SCH-92.11] D.C. Schmidt. "IPC SAP, A Family of Object-Oriented Interfaces for Local and Remote Interprocess Communication. C++ Report, vol. 4, Nov/ Dec 1992. 
[SCH-95.11] D.C. Schmidt, "Acceptor: a Design Pattern for Passively Initializing Network Services," C++Report, Nov./Dec. 1995.

[SCH-95.1] D.C. Schmidt and S. Vinoski. "Introduction to Distributed Object Computing", C++ Report, Jan. 1995.

[SCH-95.2] D.C. Schmidt and S. Vinoski. "Modeling Distributed Object Applications", C++ Report, Feb. 1995.

[SCH-95.5] DC. Schmidt and S. Vinoski, "Comparing Alternative Client-side Distributed Programming Techniques". SIGS C++ Report, May 1995.

[SCH-95.6] D.C.Schmidt, T.H. Harrison, and E. Al-Shaer, "Object-Oriented Components for High-speed Network Programming," in Proceedings of the Ist Conference on Object-Oriented Technologies, (Monterey, CA), USENIX, June 1995.

[SCH-95.10] D.C. Schmidt and S. Vinoski, "Comparing Alternative Server Programming Techniques", SIGS C++ Report, Oct. 1995.

[SCH-95.31] D.C. Schmidt, "An OO Encapsulation of Lightweight OS Concurrency Mechanisms in the ACE Toolkit", Technical Report Number WUCS-9531, Washington University, St. Louis.

[SCH-96.1] D.C. Schmidt, "Connector: a Design Pattern for Actively Initializing Network Services," C++ Report, vol. 8, January 1996.

[SCH-96.2] D.C. Schmidt and S. Vinoski, "Comparing Alternative Programming Techniques for Multi-threaded Servers", SIGS C++Report, Feb. 1996.

[SCH-96.4] D.C. Schmidt and S. Vinoski, "Comparing Alternative Programming Techniques for Multi-threaded CORBA Servers", SIGS C++ Report, April 1996. 
[SCH-96.6] D.C. Schmidt and S. Vinoski, "Comparing Alternative Programming Techniques for Multi-threaded CORBA Servers", SIGS C++ Report, June 1996.

[SIE-96] J. Siegel, "CORBA Fundamentals and Programming", John Wiley \& Sons, Inc, 1996, 605 Third Avenue, New York, N.Y. 10158-0012.

[SME-91] B. Smetaniuk, "Distributed operation of the X.500 directory", Computer Networks and ISDN Systems 21(1991) 17-40, North Holland.

[SOM-97] G. Somadder, "OO Multi-Threaded Servers: Design with reusable components, Performance Measurement and Analysis", M.Eng. Thesis, Carleton University, Ottawa, Canada, March 1997.

[STA-95] M. Stal, "The Broker Architectural Framework", Object-Oriented Programming Systems, Languages and Applications (OOPSLA'95), Maitland, Florida, U.S.A.

[STE-92] W.R. Stevens. "Advanced Programming in the Unix Environment". Reading, Massachusetts. Addison-Wesley, 1992.

[STR-91] Bjarne Stroustrup, "The C+ Programming Language", 2 nd Edition. Addison-Wesley, 1991.

[TAN-95] A.Tanenbaum, "Distributed Operating Systems", Prentice-Hall Inc., Englewood Cliffs, N.J. 07632, 1995.

[VIN-93] S. Vinoski, "Distributed Object Computing with CORBA", C++Report, vol.5 July/August, 1993. 
Appendix A: Supplementary Results for

the Message Size Set of

\author{
Experiments
}




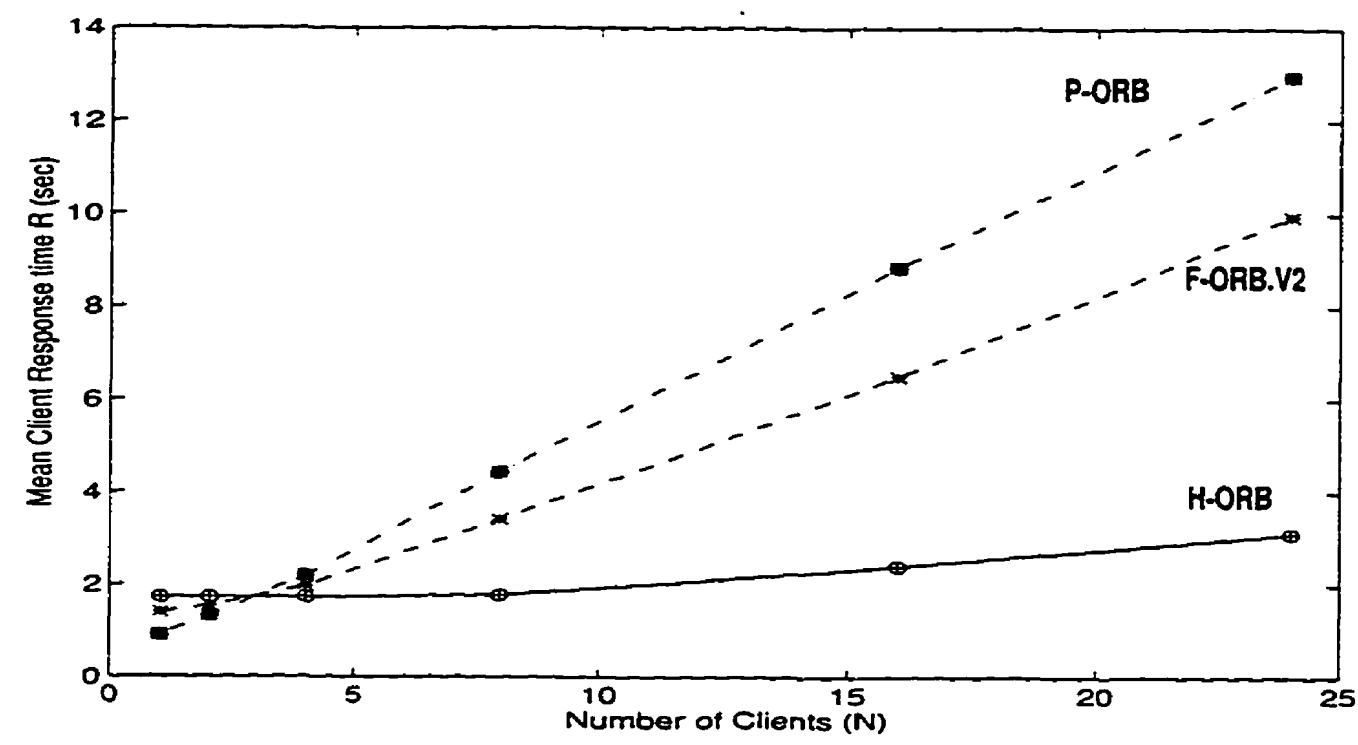

Figure A-1: The Response Time of the H-ORB, the F-ORB.V2 and the $P$-ORB with $L=4800$ Bytes, and No Cloning.

Degree of Cloning $=1, D=200 \mathrm{~ms}, L=4800$ Bytes, $S_{A}=10 \mathrm{~ms}, S_{B}=15 \mathrm{~ms}$

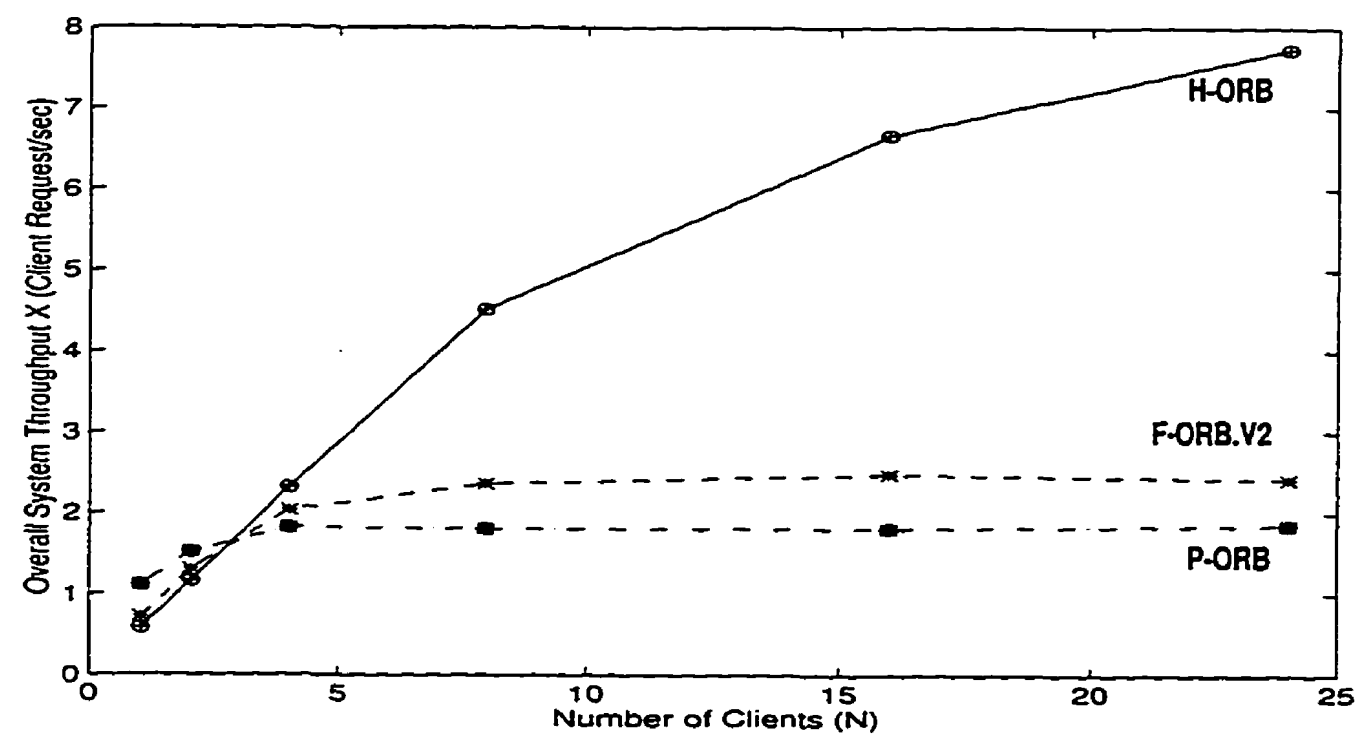

Figure A-2: The Throughput of the H-ORB, the F-ORB.V2 and the P$O R B$ with $L=4800$ Bytes, and No Cloning.

Degree of Cloning $=1, D=200 \mathrm{~ms}, L=4800$ Bytes, $S_{A}=10 \mathrm{~ms}, S_{B}=15 \mathrm{~ms}$ 


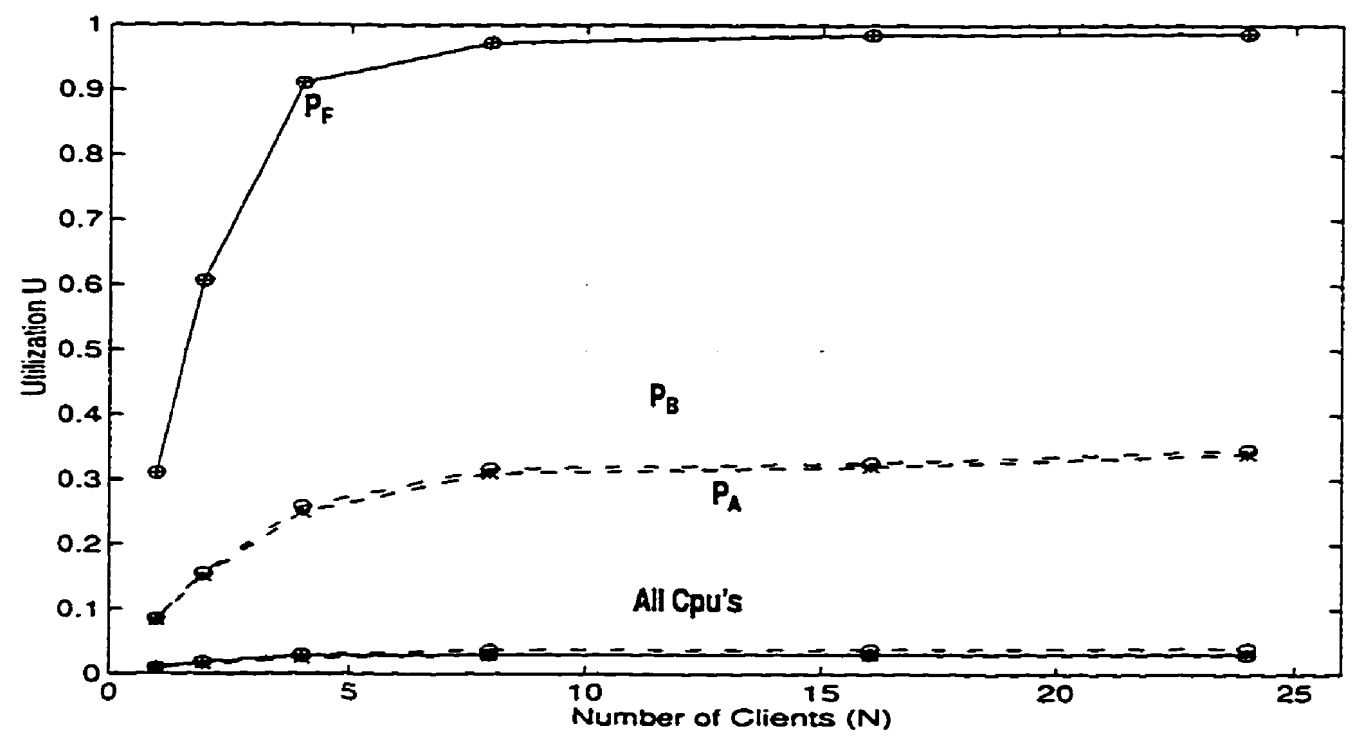

Figure A-3: Cpu \&Process Utilizations of the F-ORB.V2 with $L=4800$ Bytes and No cloning.

$P_{A}=$ Process Util. of Server $A, C P U_{A}=C p u$ Util of Server $A . P_{p}=$ Process Util. of p-agent. Degree of Cloning $=1, D=200 \mathrm{~ms}, L=4800$ Bytes, $S_{A}=10 \mathrm{~ms}, S_{B}=15 \mathrm{~ms}$ 


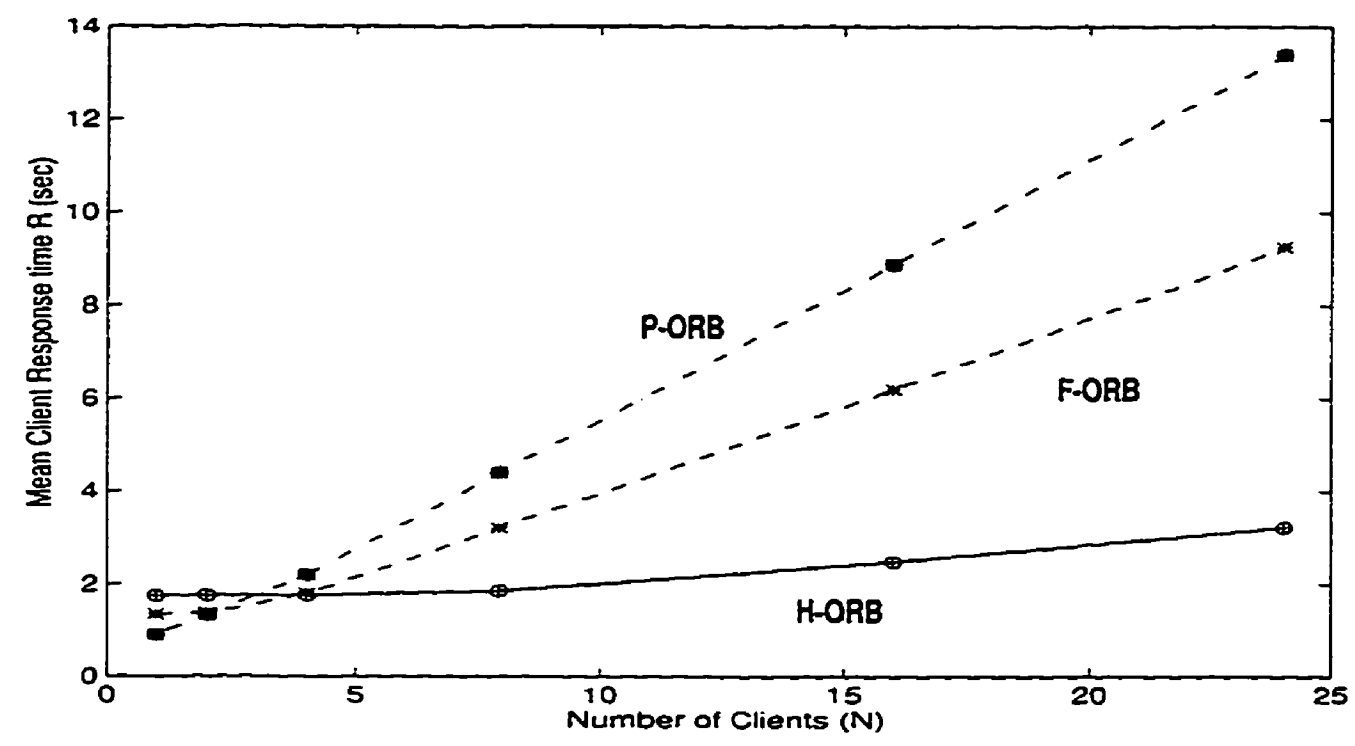

Figure A-4: The Response Time of the H-ORB, the F-ORB and the PORB with $L=9600$ Bytes, and No Cloning.

Degree of Cloning $1, D=200 \mathrm{~ms}, L=9600$ Bytes, $S_{A}=10 \mathrm{~ms}, S_{B}=15 \mathrm{~ms}$

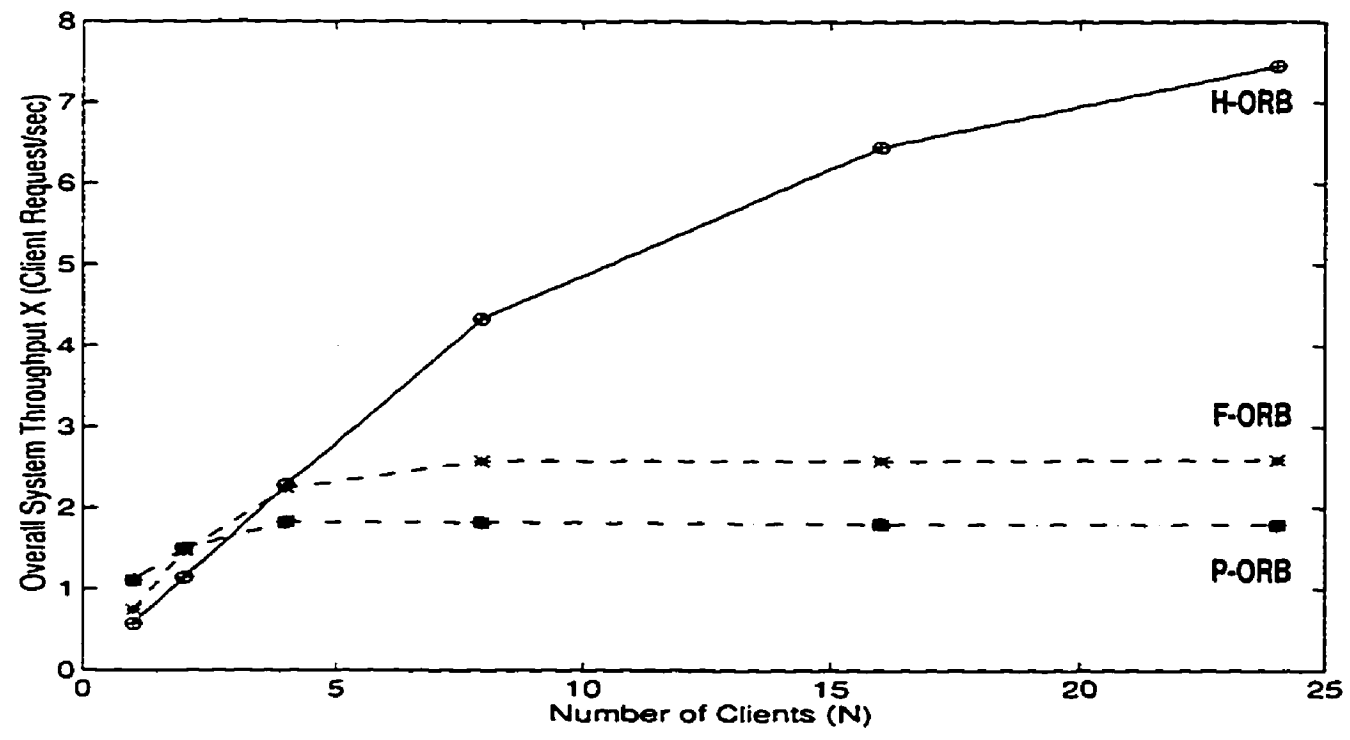

Figure A-5: The Throughput of the H-ORB, the F-ORB and the P$O R B$ with $L=9600$ Bytes, and No Cloning.

Degree of Cloning $=1, D=200 \mathrm{~ms}, L=9600$ Bytes, $S_{A}=10 \mathrm{~ms}, S_{B}=15 \mathrm{~ms}$ 


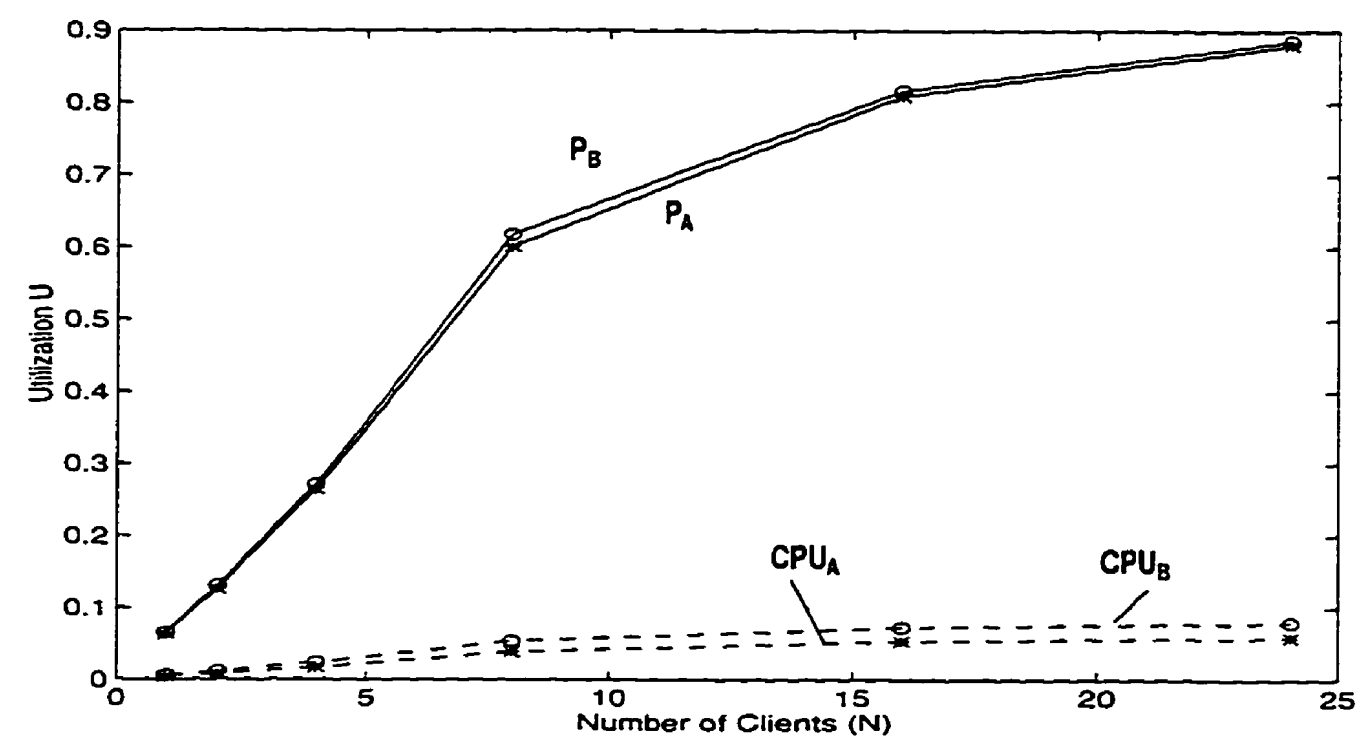

Figure A-6: Cpu and Process Utilizations of the H-ORB with $L=9600$ Bytes and No cloning.

$P_{A}=$ Process Util. of Server $A, C P U_{A}=C p u$ Util of Server $A$.

Degree of Cloning $=1, D=200 \mathrm{~ms}, L=9600$ Bytes, $S_{A}=10 \mathrm{~ms}, S_{B}=15 \mathrm{~ms}$

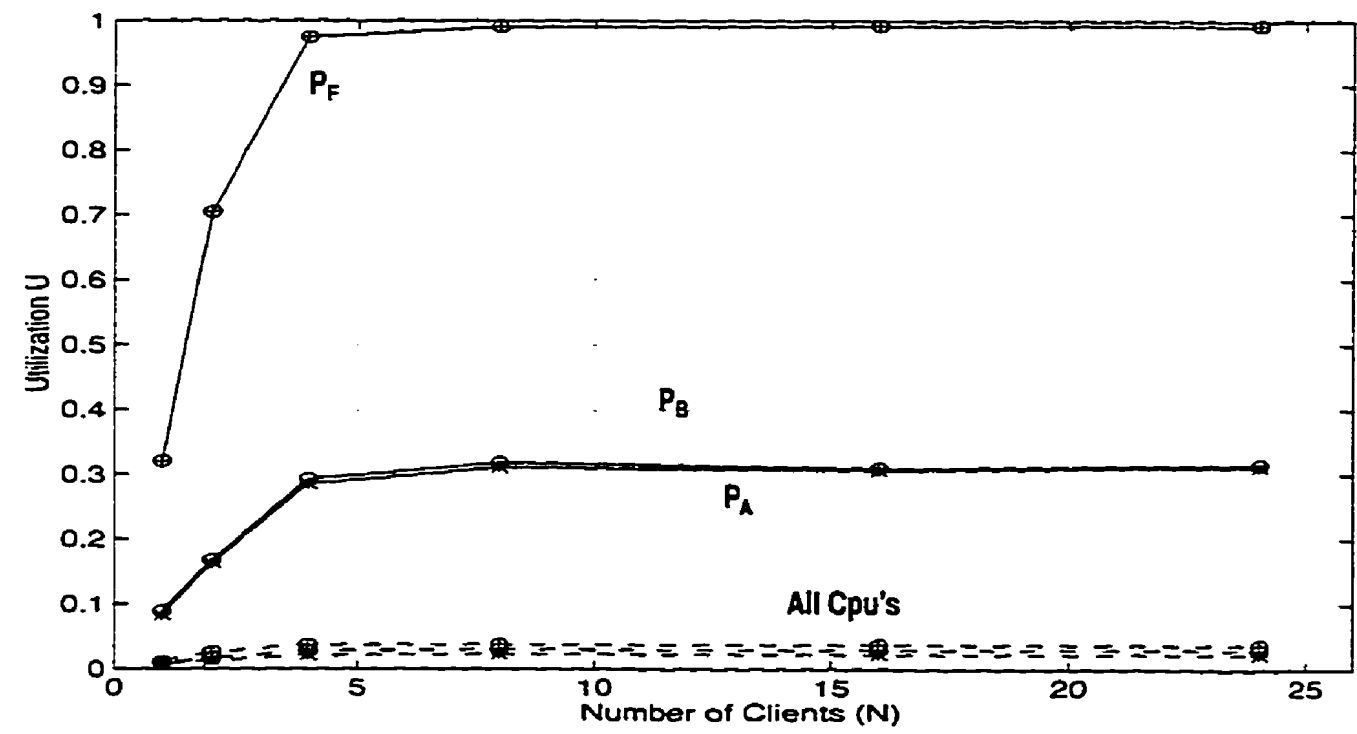

Figure A-7: Cpu \&Process Utilizations of the F-ORB with L=9600 Bytes and No cloning.

$P_{A}=$ Process Util. of Server $A, C P U_{A}=C$ u Util of Server $A . P_{F}=$ Process Util. of f-agent.

Degree of Cloning $=1, D=200 \mathrm{~ms}, L=9600$ Bytes, $S_{A}=10 \mathrm{~ms}, S_{B}=15 \mathrm{~ms}$ 


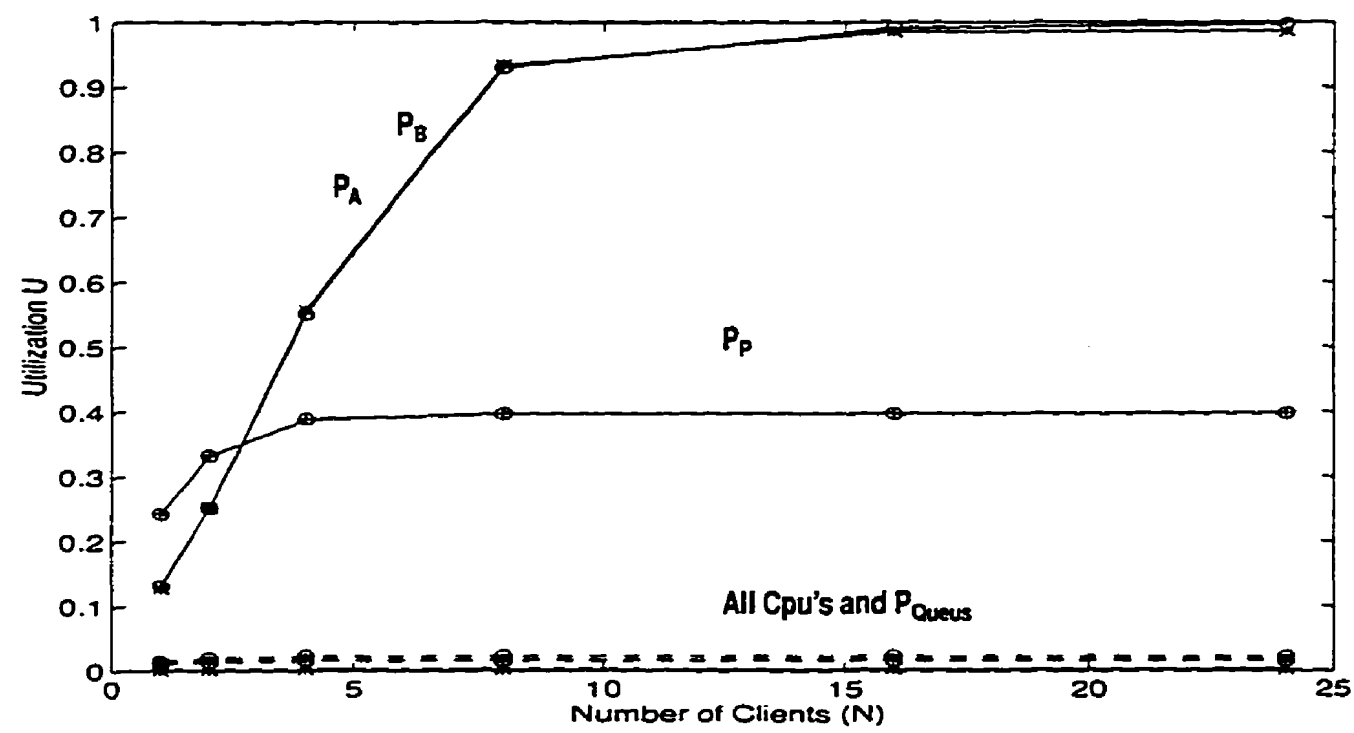

Figure A-8: Cpu \& Process Utilizations of the P-ORB with $L=9600$ Bytes and No cloning.

$P_{A}=$ Process Util. of Server $A, C P U_{A}=C p u$ Util of Server A.P $=$ Process Util. of p-agent. $P_{\text {Queues }}=$ Process Util. of dispatcher, Degree of Cloning $=1, D=200 \mathrm{~ms}, L=9600$ Bytes, $S_{A}=10 \mathrm{~ms}, S_{B}=15 \mathrm{~ms}$ 


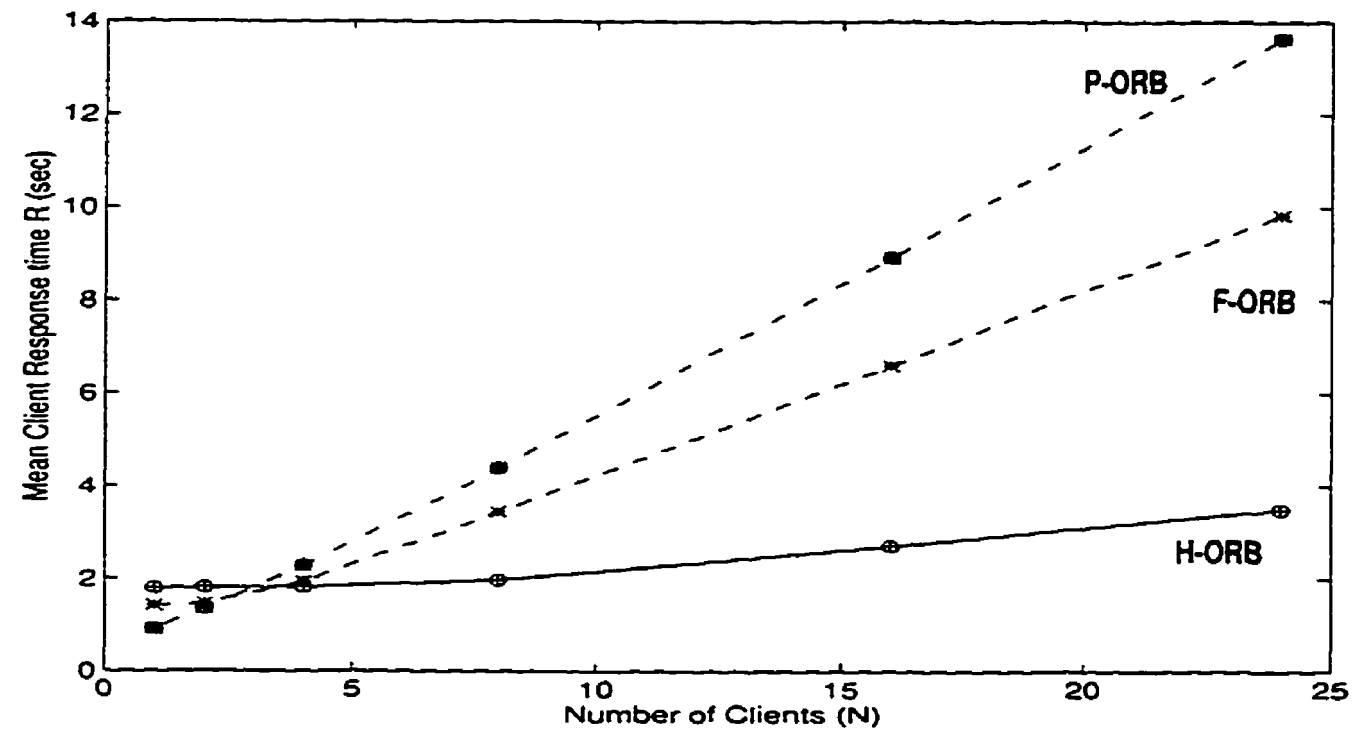

Figure A-9: The Response Time of the H-ORB, the F-ORB and the PORB with $L=19200$ Bytes, and No Cloning.

Degree of Cloning $=1, D=200 \mathrm{~ms}, L=19200$ Bytes, $S_{A}=10 \mathrm{~ms}, S_{B}=15 \mathrm{~ms}$

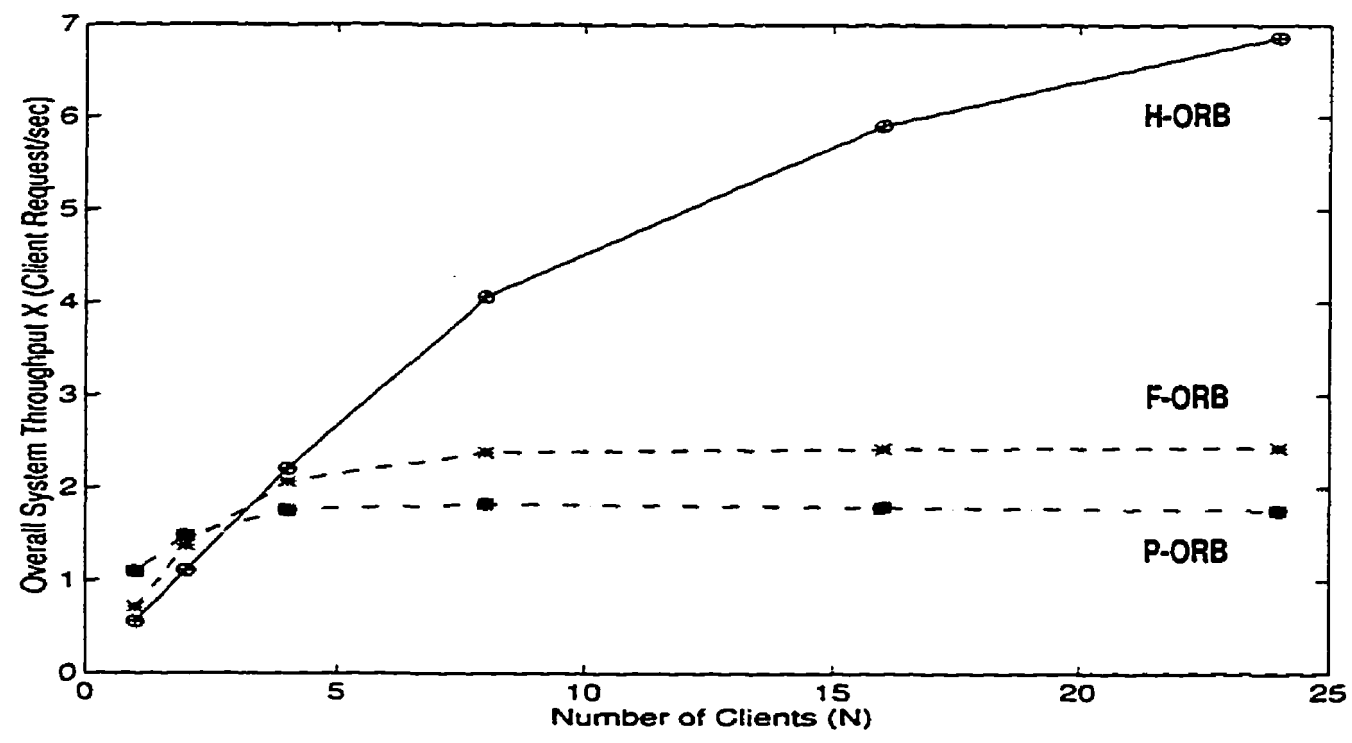

Figure A-10: The Throughput of the H-ORB, the F-ORB and the PORB with $L=19200$ Bytes, and No Cloning.

Degree of Cloning $=1, D=200 \mathrm{~ms}, L=19200$ Byres, $S_{A}=10 \mathrm{~ms}, S_{B}=15 \mathrm{~ms}$ 


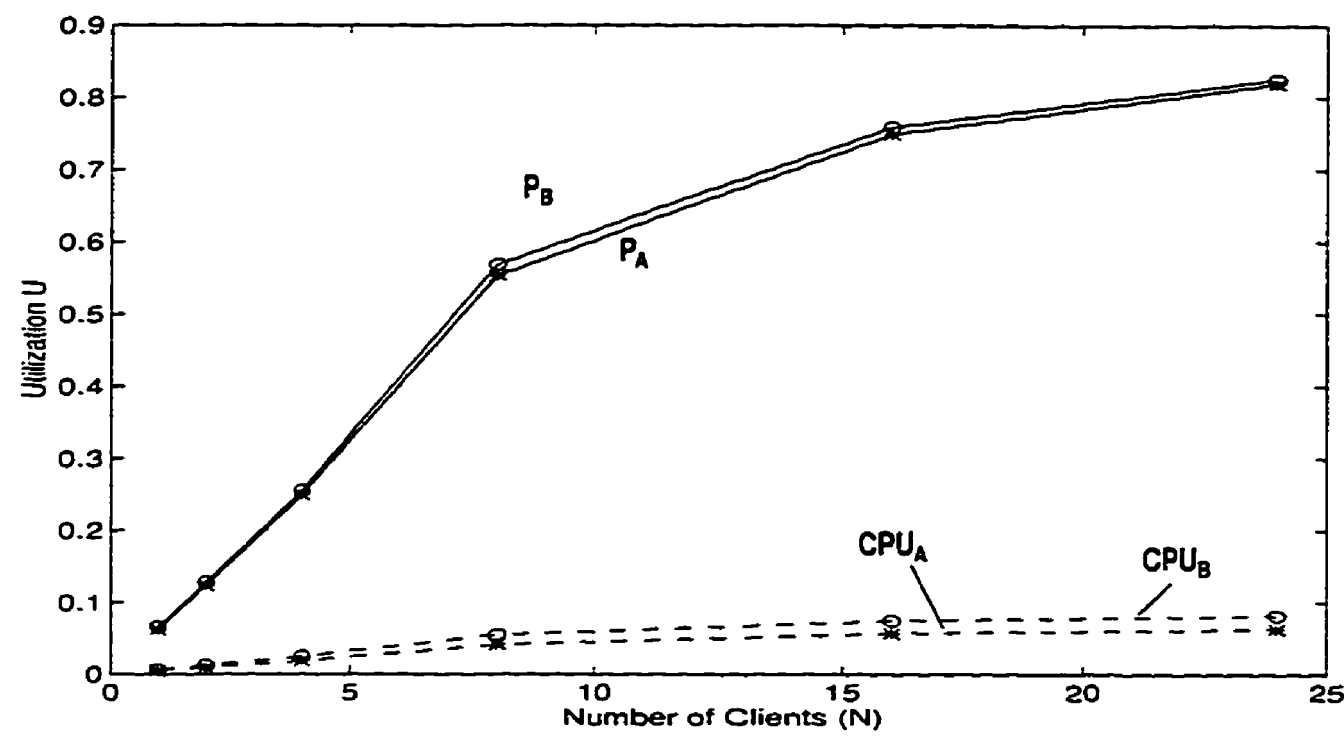

Figure A-11: Cpu and Process Utilizations of the H-ORB with $L=19200$ Bytes and No cloning.

$P_{A}=$ Process Util. of Server $A, C P U_{A}=C p u$ Util of Server $A$.

Degree of Cloning $=1, D=200 \mathrm{~ms}, \mathrm{~L}=19200$ Bytes, $S_{A}=10 \mathrm{~ms}, S_{B}=15 \mathrm{~ms}$

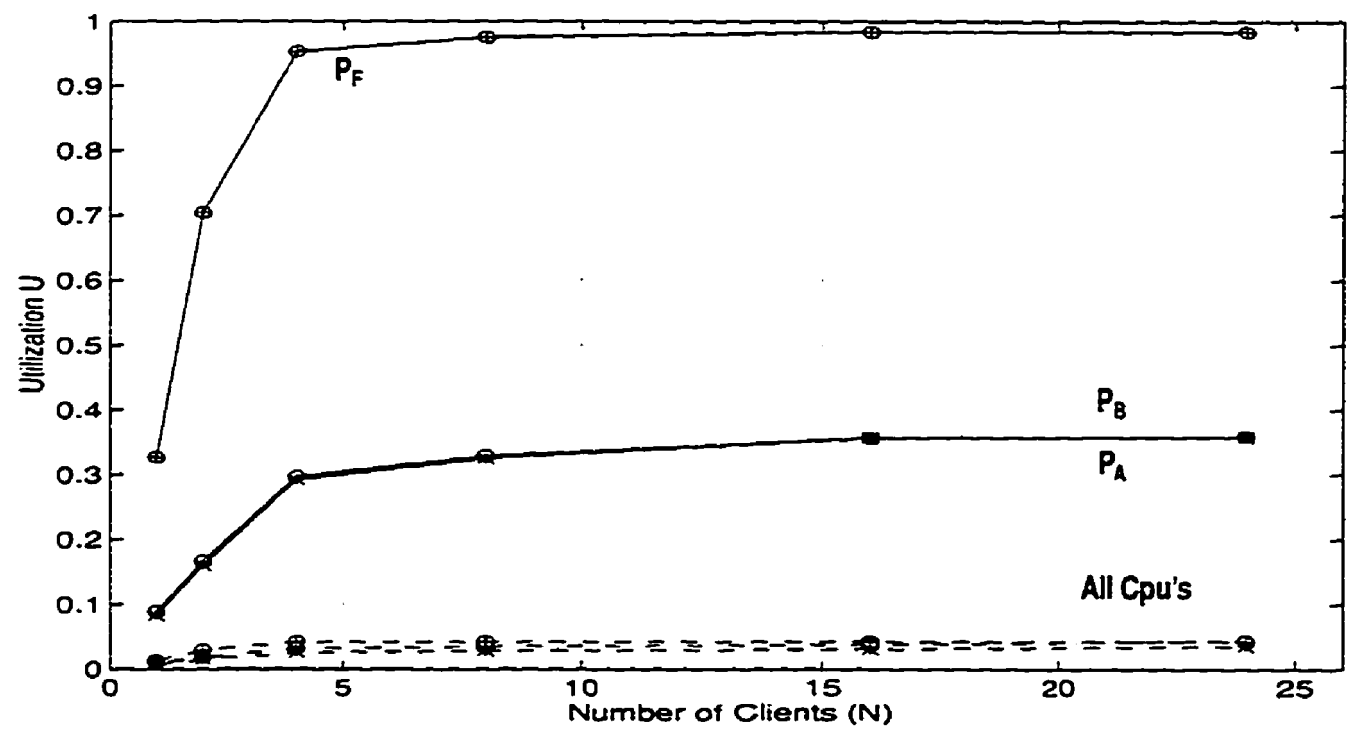

Figure A-12: Cpu \& Process Utilizations of the F-ORB with $L=19200$ Bytes and No cloning.

$P_{A}=$ Process Util. of Server A, CPU $U_{A}=C p u$ Util of Server A.P $P_{F}=$ Process Util. of f-agent.

Degree of Cloning $=1, D=200 \mathrm{~ms}, L=19200$ Bytes, $S_{A}=10 \mathrm{~ms}, S_{B}=15 \mathrm{~ms}$ 


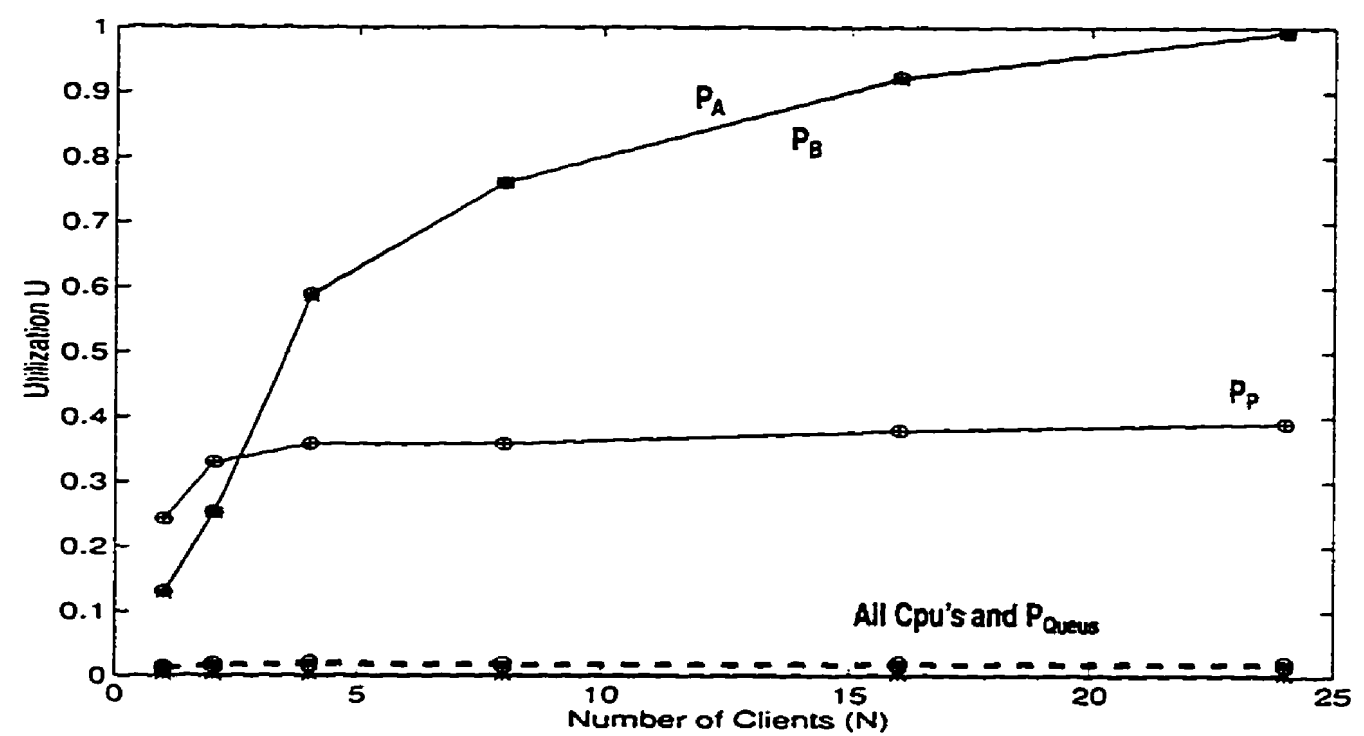

Figure A-13: Cpu \& Process Utilizations of the P-ORB with $L=19200$ Bytes and No Cloning.

$P_{A}=$ Process Util. of Server A. CPU $U_{A}=C p u$ Util of Server A.P $P_{P}=$ Process Util. of p-agent. $P_{\text {Queues }}=$ Process Util. of dispatcher, Degree of Cloning $=1, D=200 \mathrm{~ms}, L=19200$ Bytes, $\mathrm{S}_{\mathrm{A}}=10 \mathrm{~ms}, \mathrm{~S}_{\mathrm{B}}=15 \mathrm{~ms}$ 


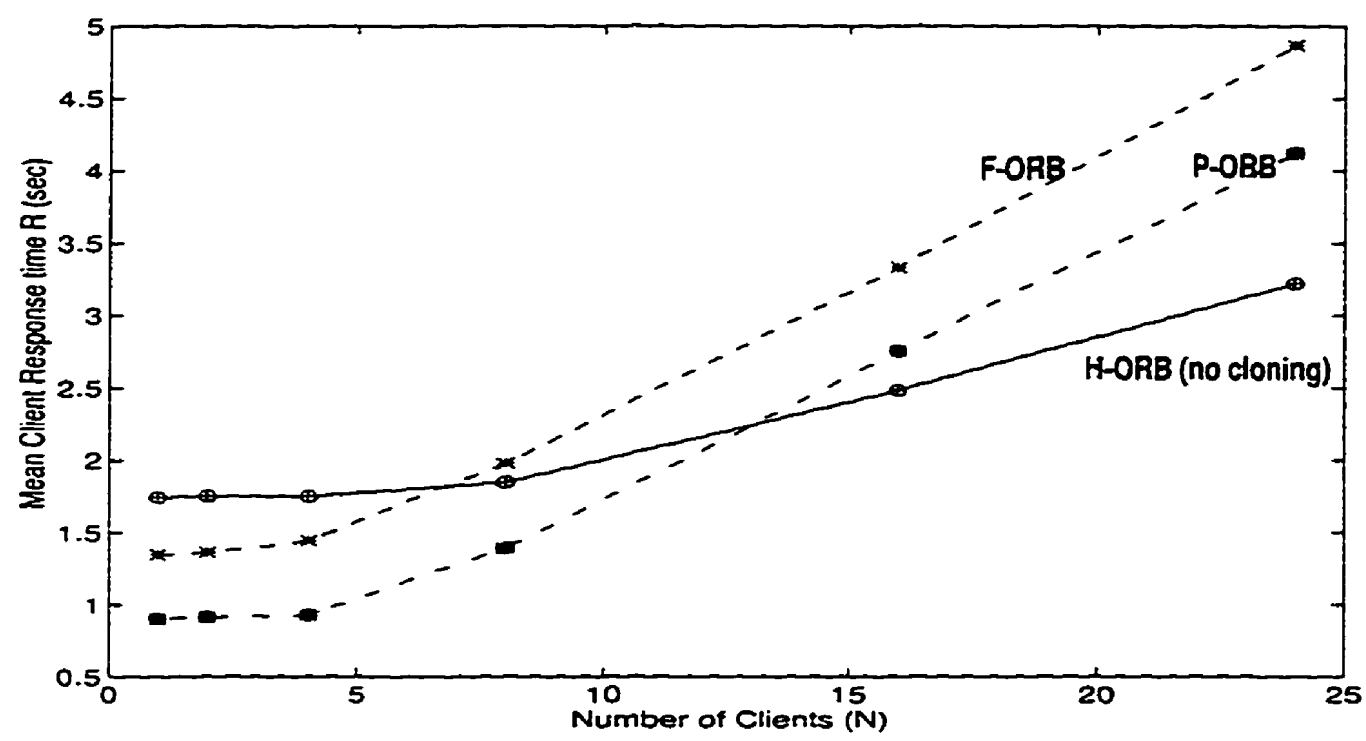

Figure A-14: The Response Time of the H-ORB, the F-ORB and the P$O R B$ with $L=9600$ Bytes, and 4 Cloning Degrees.

Degree of Cloning $=4, D=200 \mathrm{~ms}, L=9600$ Bytes, $S_{A}=10 \mathrm{~ms}, S_{B}=15 \mathrm{~ms}$

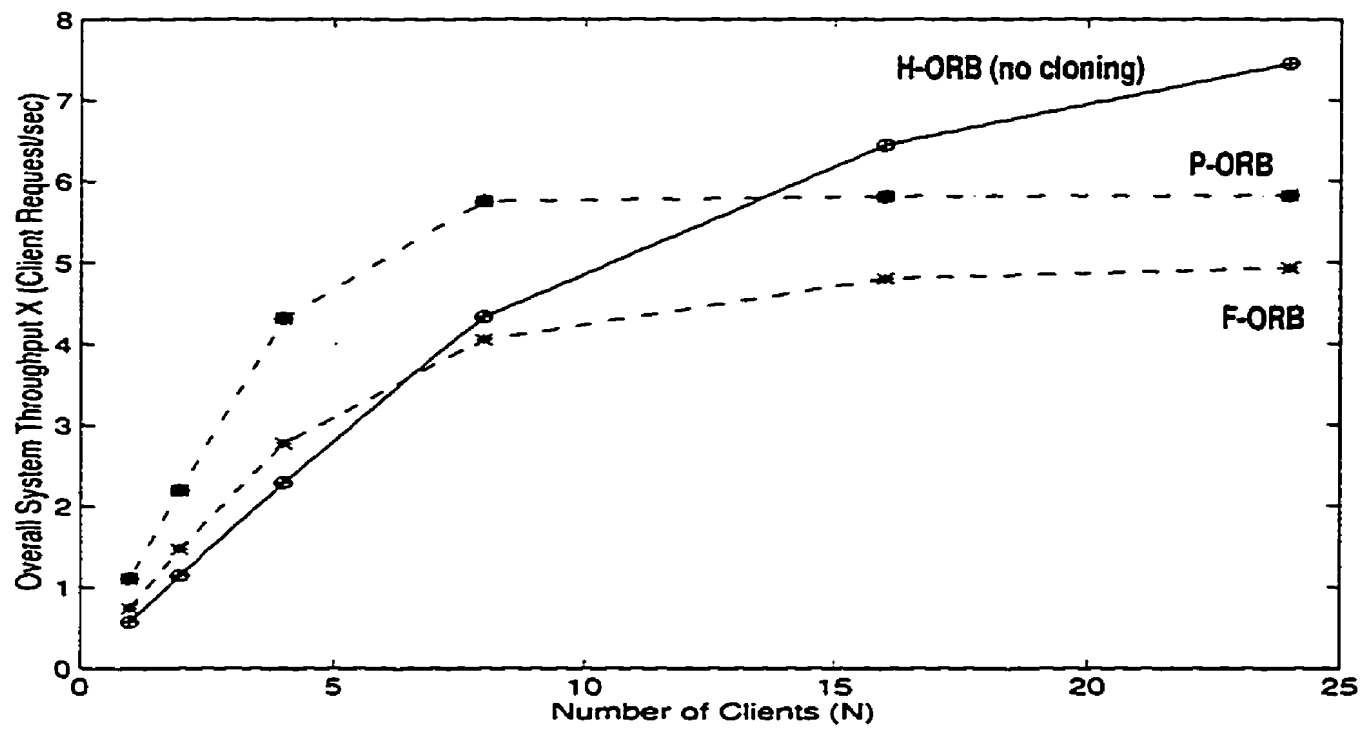

Figure A-15: The Throughput of the H-ORB, the F-ORB and the PORB with $L=9600$ Bytes, and 4 Cloning Degrees.

Degree of Cloning $=4, D=200 \mathrm{~ms}, \mathrm{~L}=9600$ Bytes, $S_{A}=10 \mathrm{~ms}, S_{B}=15 \mathrm{~ms}$ 


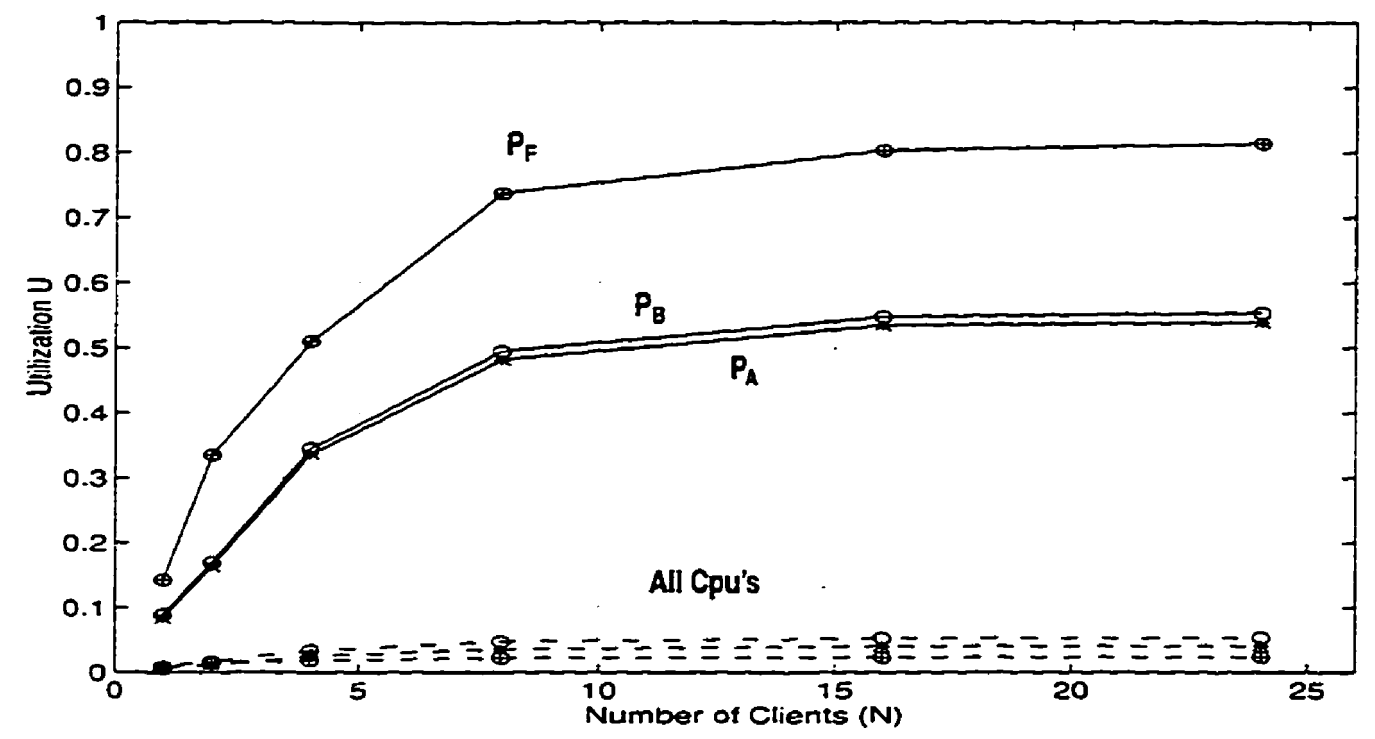

Figure A-16: Cpu \&Process Utilizations of the F-ORB with $L=9600$ Bytes and 4 Cloning Degrees.

$P_{A}=$ Process Util. of Server A. $C P U_{A}=C p u$ Util of Server A. $P_{F}=$ Process Util. of f-agent. Degree of Cloning $=4, D=200 \mathrm{~ms}, L=9600$ Bytes, $S_{A}=10 \mathrm{~ms}, S_{B}=15 \mathrm{~ms}$

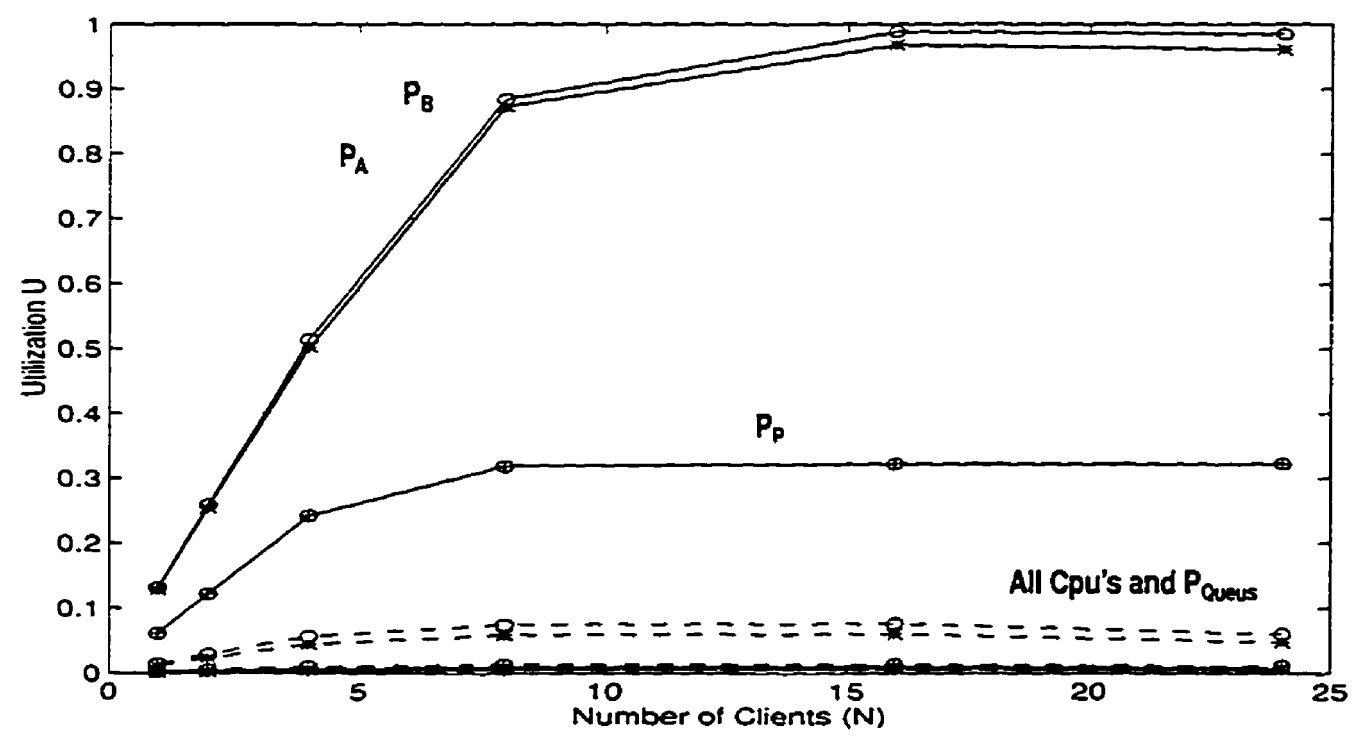

Figure A-17: Cpu \&Process Utilizations of the P-ORB with $L=9600$ Bytes and 4 Cloning Degrees.

$P_{A}=$ Process Util. of Server $A, C P U_{A}=C p u$ Util of Server A.P $P=$ Process Util. of p-agent. $P_{\text {Queres }}=$ Process Util. of dispatcher, Degree of Cloning $=4, D=200 \mathrm{~ms}, L=9600$ Bytes, $S_{A}=10 \mathrm{~ms}, S_{B}=15 \mathrm{~ms}$ 


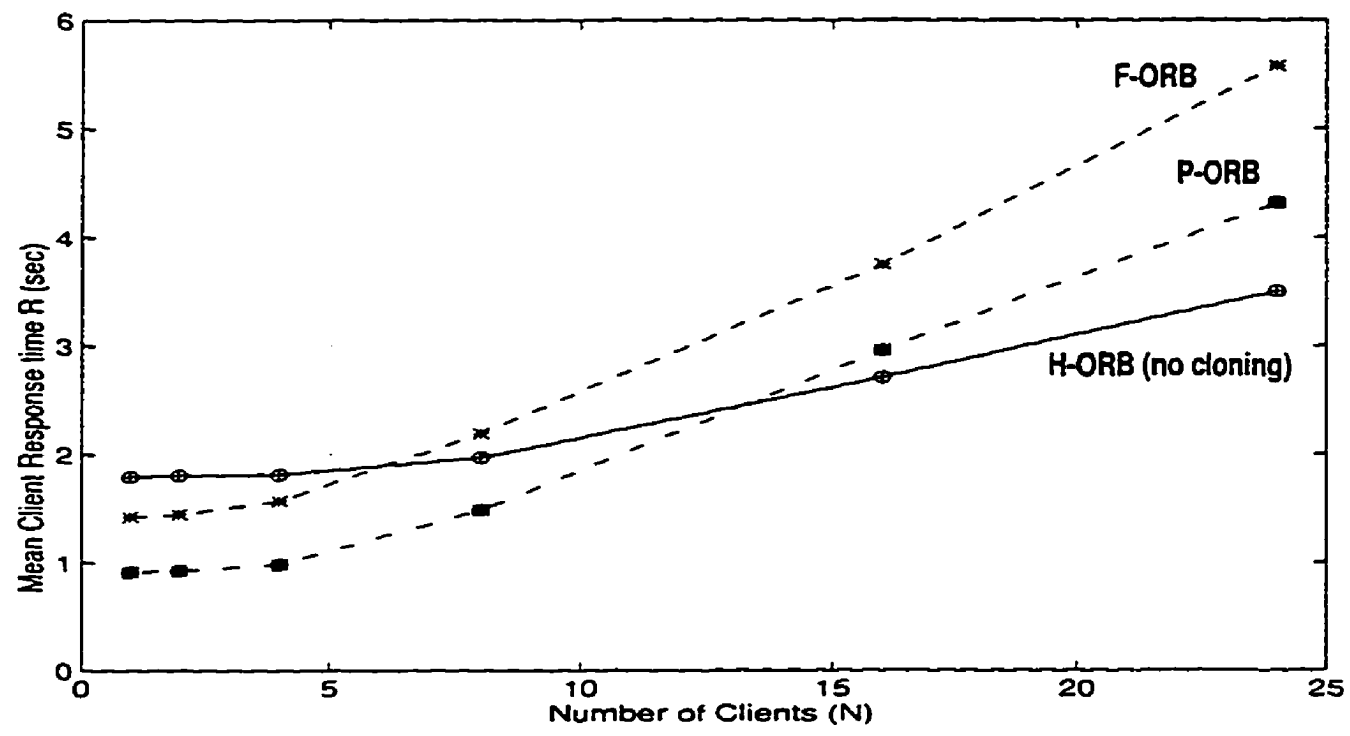

Figure A-18: The Response Time of the H-ORB, the F-ORB and the PORB with $L=19200$ Bytes, and 4 Cloning Degrees.

Degree of Cloning $=4, D=200 \mathrm{~ms}, L=19200$ Bytes, $S_{A}=10 \mathrm{~ms}, S_{B}=15 \mathrm{~ms}$

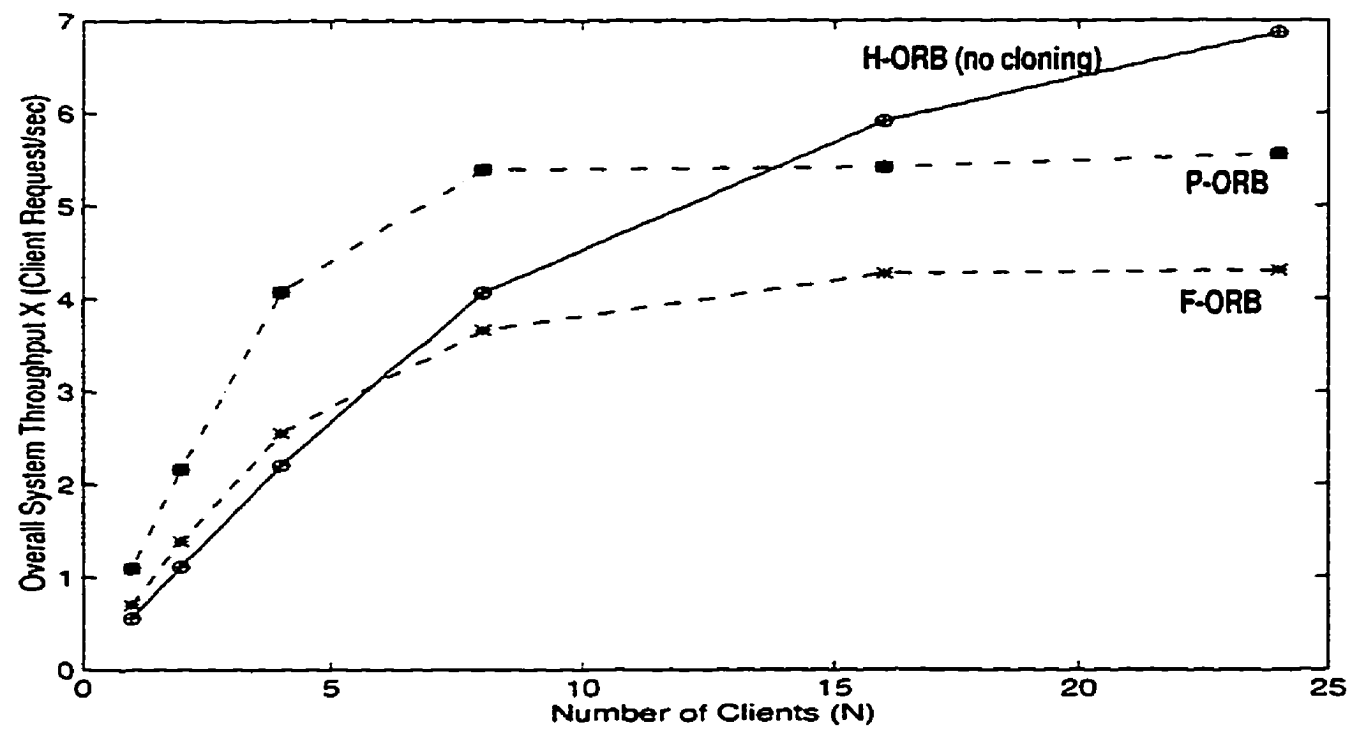

Figure A-19: The Throughput of the H-ORB, the F-ORB and the P-

ORB with $L=19200 B y t e s$, and 4 Cloning Degrees.

Degree of Cloning $=4, D=200 \mathrm{~ms}, L=19200$ Bytes, $S_{A}=10 \mathrm{~ms}, S_{B}=15 \mathrm{~ms}$ 


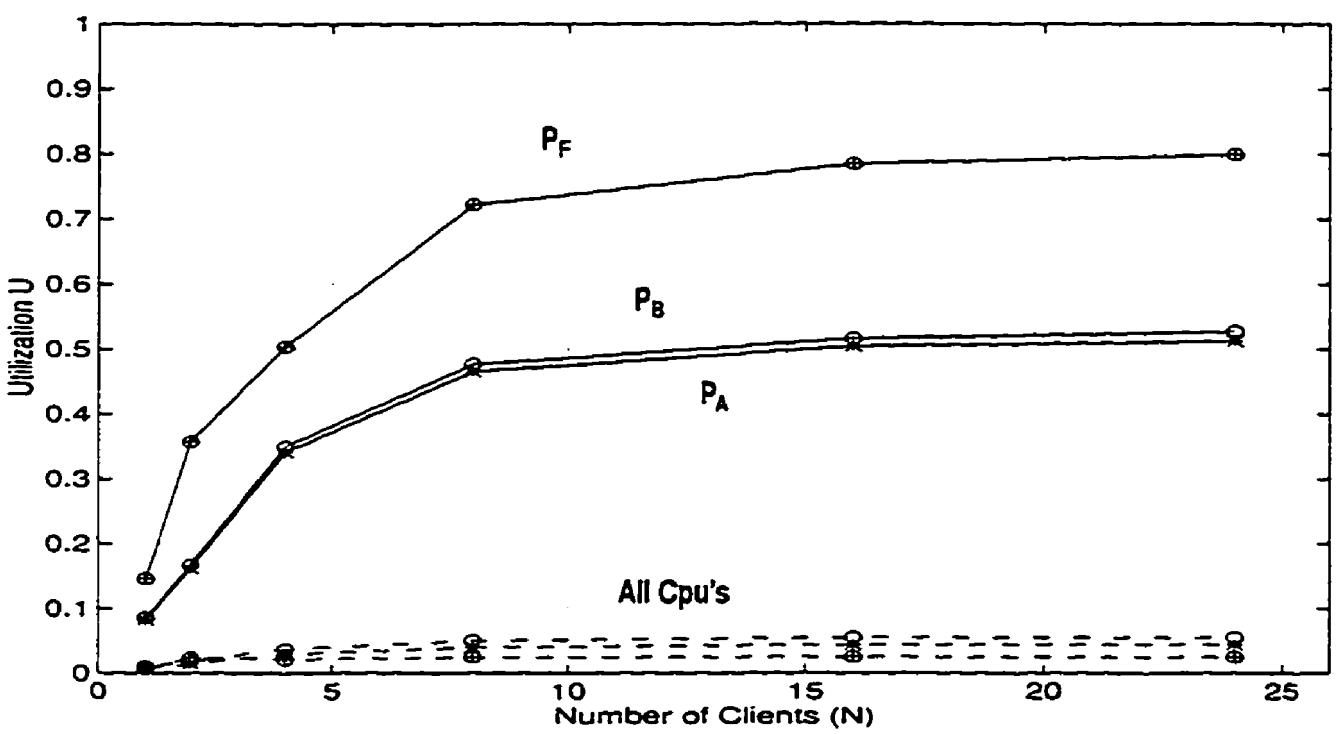

Figure A-20: Cpu \&Process Utilizations of the F-ORB with $L=19200$ Bytes and 4 Cloning Degrees.

$P_{A}=$ Process Util. of Server $A . C P U_{A}=C p u$ Util of Server A.P $P_{F}=$ Process Util. of f-agent. Degree of Cloning $=4, D=200 \mathrm{~ms}, L=19200$ Bytes, $S_{A}=10 \mathrm{~ms}, S_{B}=15 \mathrm{~ms}$

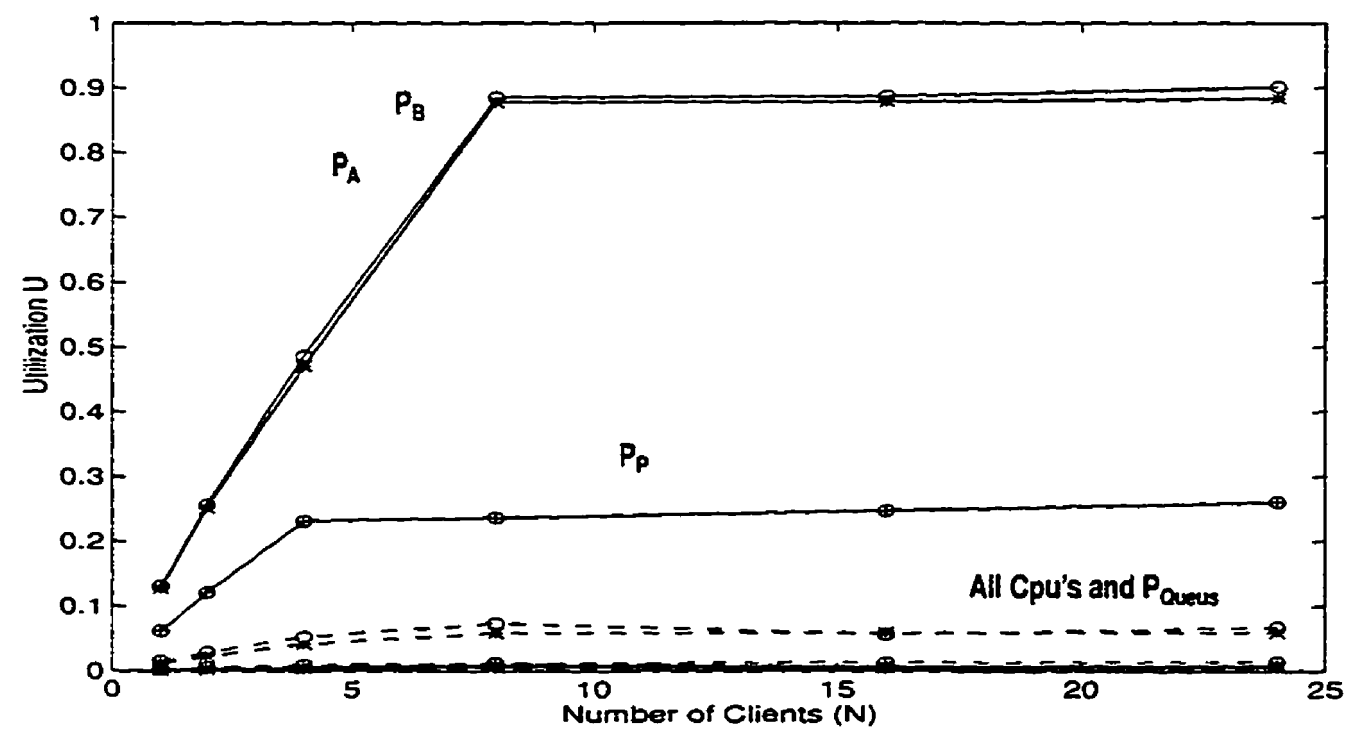

Figure A-21: Cpu \&Process Utilizations of the P-ORB with L=19200 Bytes and 4 Cloning Degrees.

$P_{A}=$ Process Util. of Server A, $C P U_{A}=C_{p u}$ Util of Server A.P $P_{P}=$ Process Util. of p-agent. $P_{\text {Queues }}=$ Process Util. of dispatcher, Degree of Cloning $=4, D=200 \mathrm{~ms}, L=19200$ Bytes, $S_{A}=10 \mathrm{~ms}, S_{B}=15 \mathrm{~ms}$ 


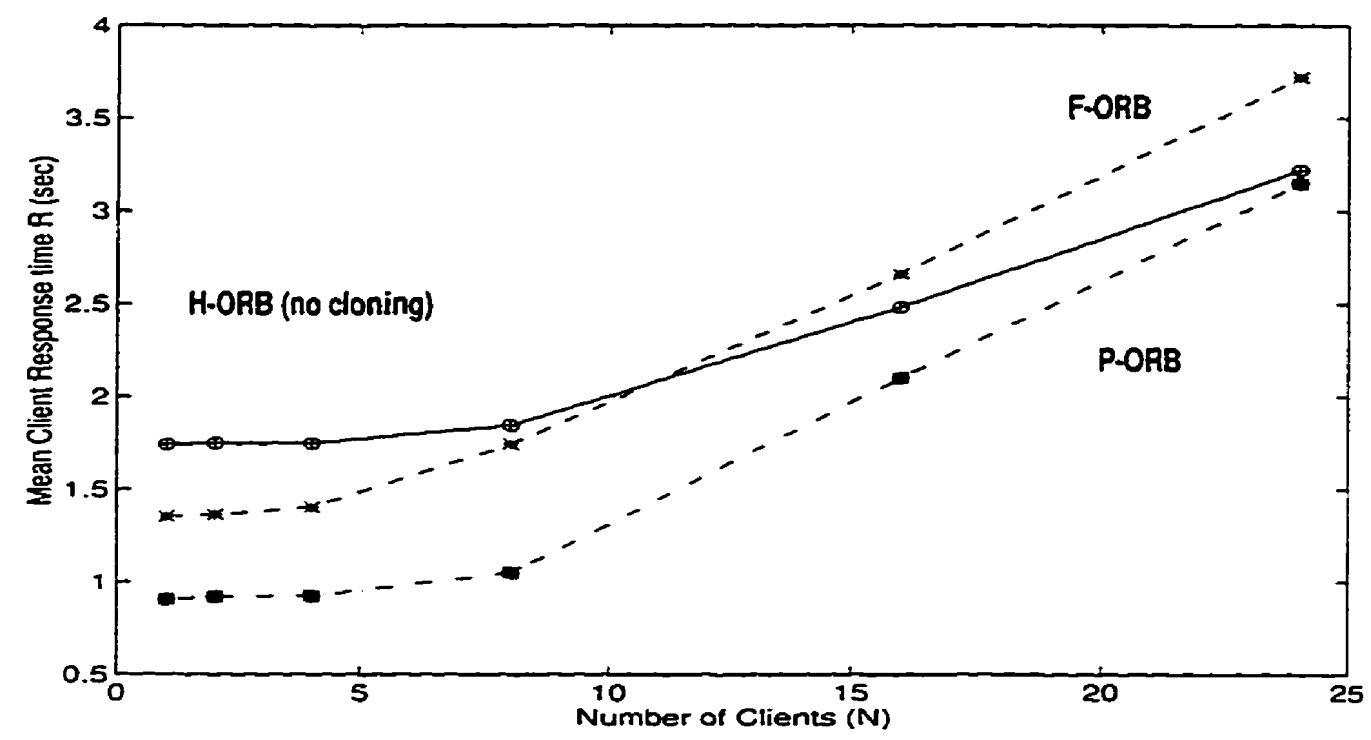

Figure A-22: The Response Time of the H-ORB, the F-ORB and the PORB with $L=9600$ Bytes, and 8 Cloning Degrees.

Degree of Cloning $=8, D=200 \mathrm{~ms}, L=9600$ Byres, $S_{A}=10 \mathrm{~ms}, S_{B}=15 \mathrm{~ms}$

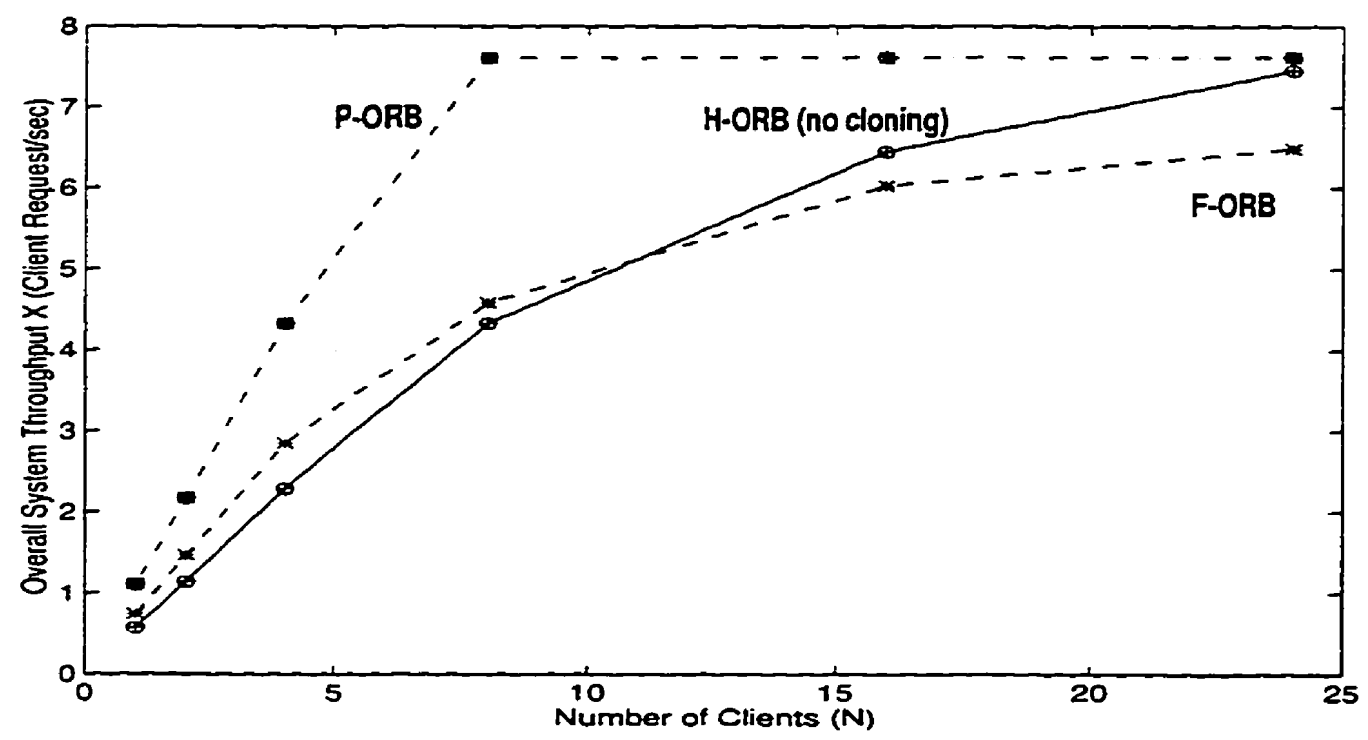

Figure A-23: The Throughput of the H-ORB, the F-ORB and the PORB with $L=9600$ Bytes, and 8 Cloning Degrees.

Degree of Cloning $=8, D=200 \mathrm{~ms}, L=9600$ Bytes, $S_{A}=10 \mathrm{~ms}, S_{B}=15 \mathrm{~ms}$ 


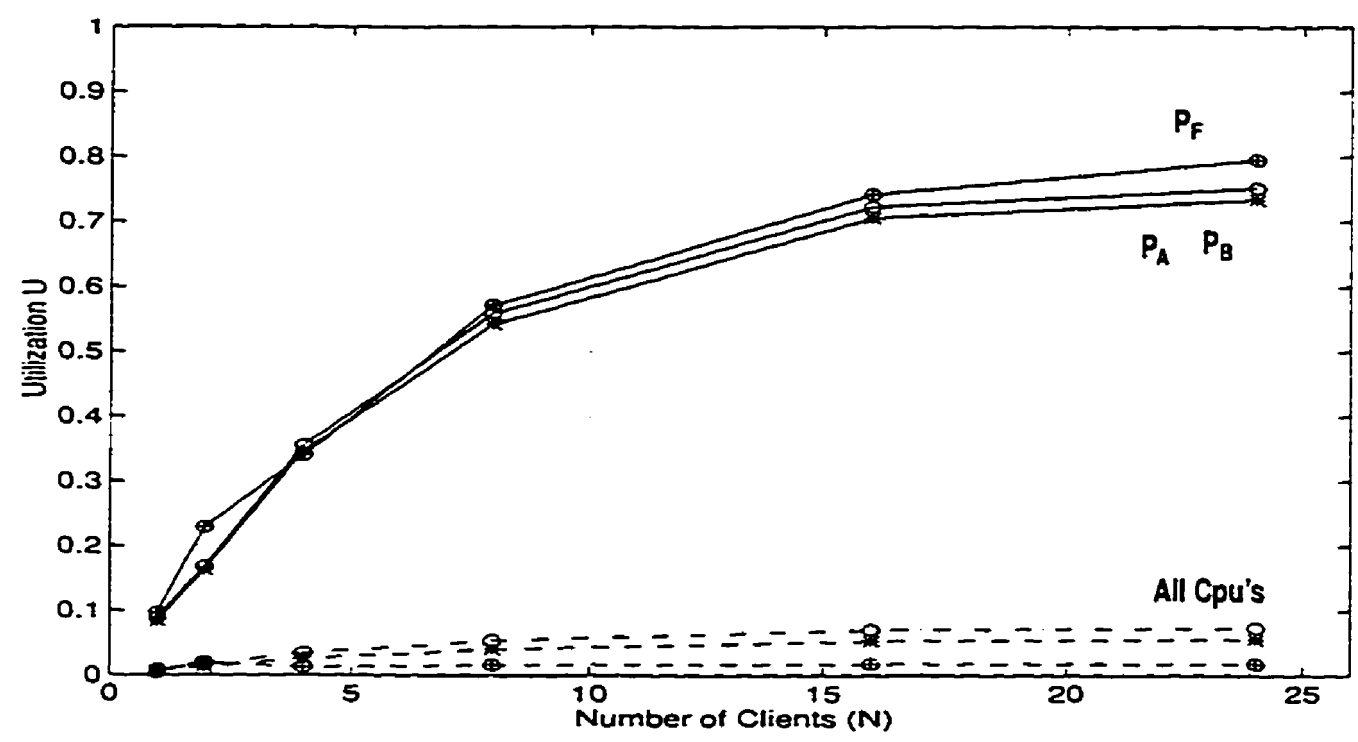

Figure A-24: Cpu \&Process Utilizations of the F-ORB with L=9600 Bytes and 8 Cloning Degrees.

$P_{A}=$ Process Util. of Server $A, C P U_{A}=C p u$ Util of Server A.P $P_{F}=$ Process Util. of f-agent. Degree of Cloning $=8, D=200 \mathrm{~ms}, \mathrm{~L}=9600$ Bytes, $S_{A}=10 \mathrm{~ms}, S_{B}=15 \mathrm{~ms}$

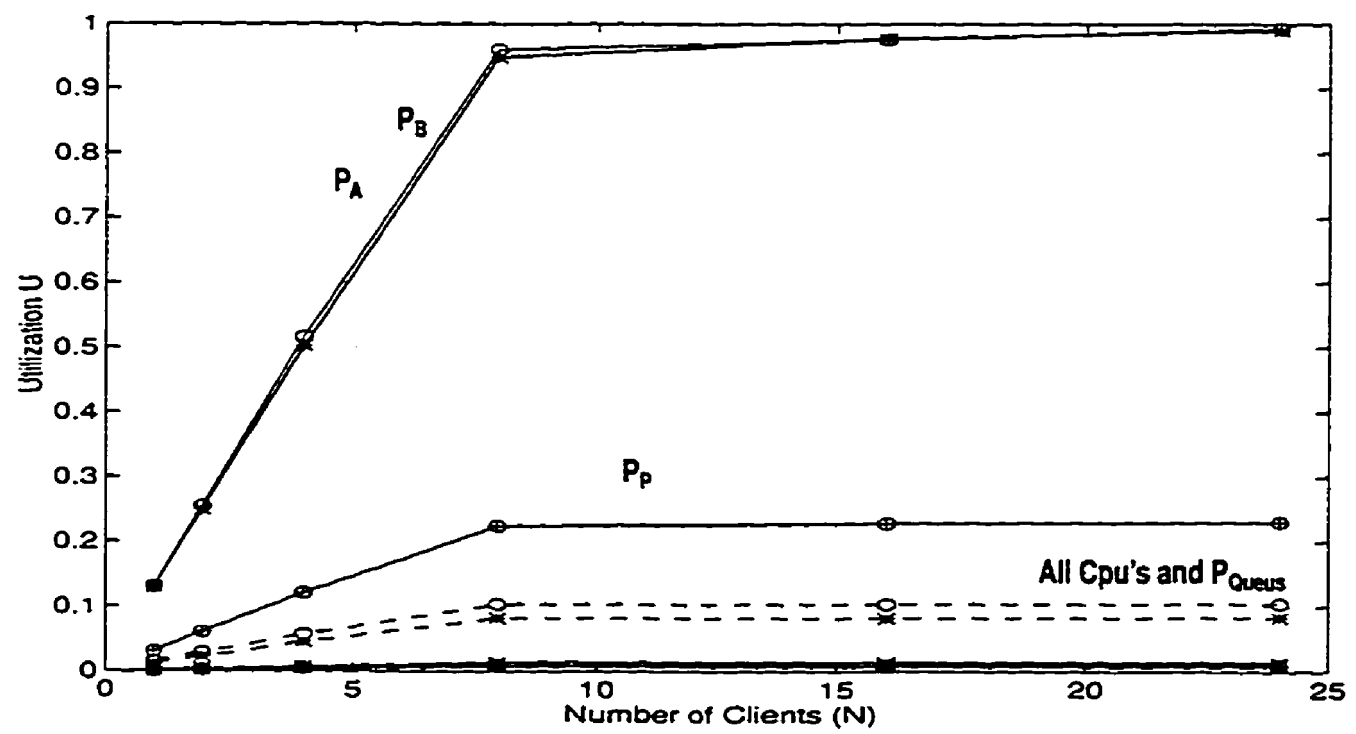

Figure A-25: Cpu \&Process Utilizations of the P-ORB with $L=9600$ Bytes and 8 Cloning Degrees.

$\mathrm{P}_{\mathrm{A}}=$ Process Util. of Server A, $C P U_{\mathrm{A}}=C$ pu Util of Server A.P $=$ Process Util. of p-agent. $P_{\text {Queues }}=$ Process Util. of dispatcher, Degree of Cloning $=8, D=200 \mathrm{~ms}, L=9600$ Bytes, $\mathrm{S}_{\mathrm{A}}=10 \mathrm{~ms}, \mathrm{~S}_{\mathrm{B}}=15 \mathrm{~ms}$ 


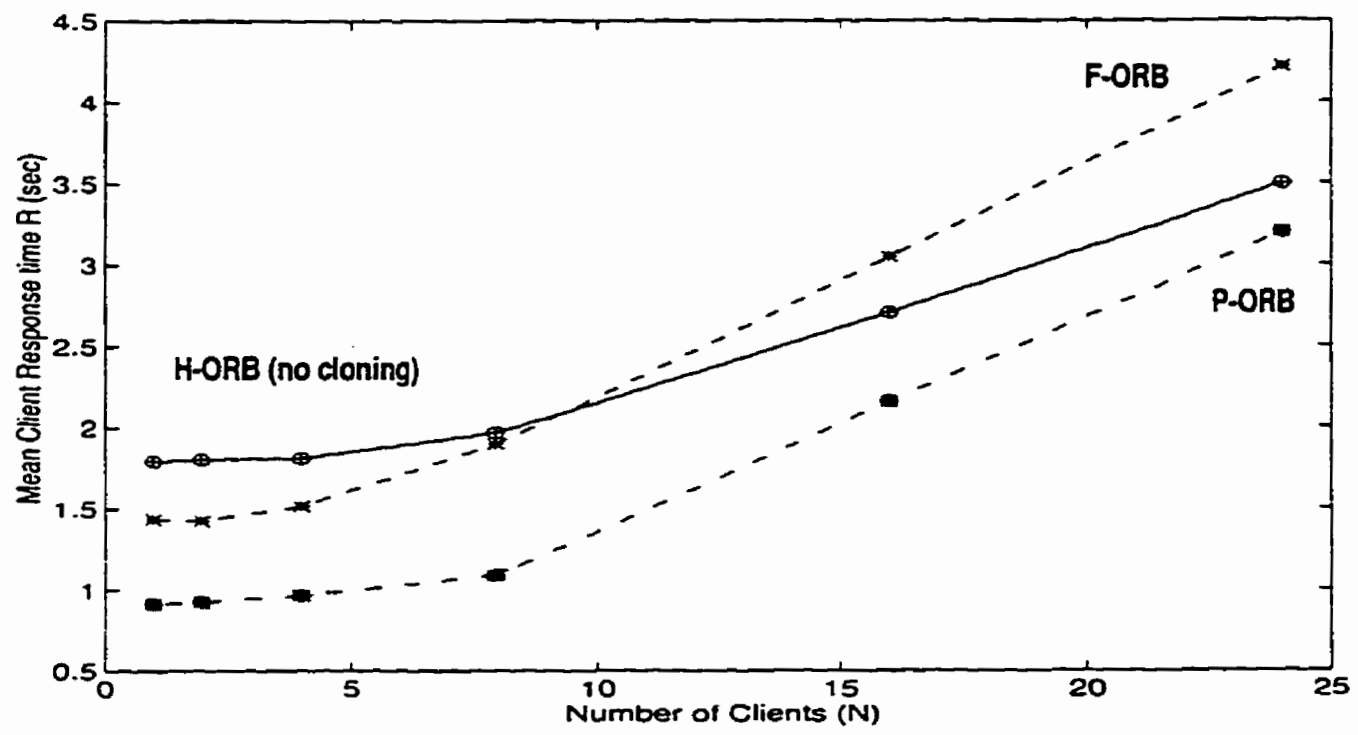

Figure A-26: The Response Time of the H-ORB, the F-ORB and the PORB with $\mathrm{L}=19200$ Bytes, and 8 Cloning Degrees.

Degree of Cloning $=8, D=200 \mathrm{~ms}, L=19200$ Bytes, $S_{A}=10 \mathrm{~ms}, S_{B}=15 \mathrm{~ms}$

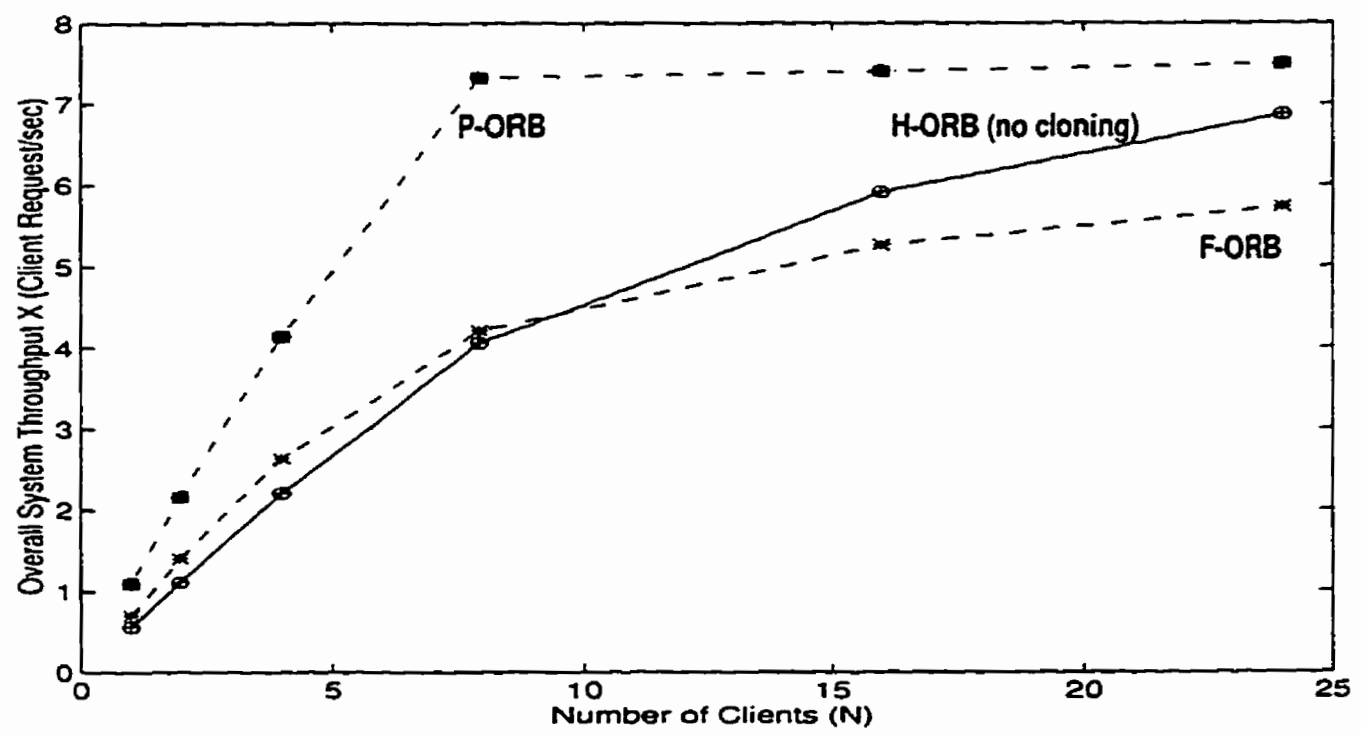

Figure A-27: The Throughput of the H-ORB, the F-ORB and the P-

$O R B$ with $L=19200$ Bytes, and 8 Cloning Degrees.

Degree of Cloning $=8, D=200 \mathrm{~ms}, \mathrm{~L}=19200$ Bytes, $S_{A}=10 \mathrm{~ms}, S_{B}=15 \mathrm{~ms}$ 


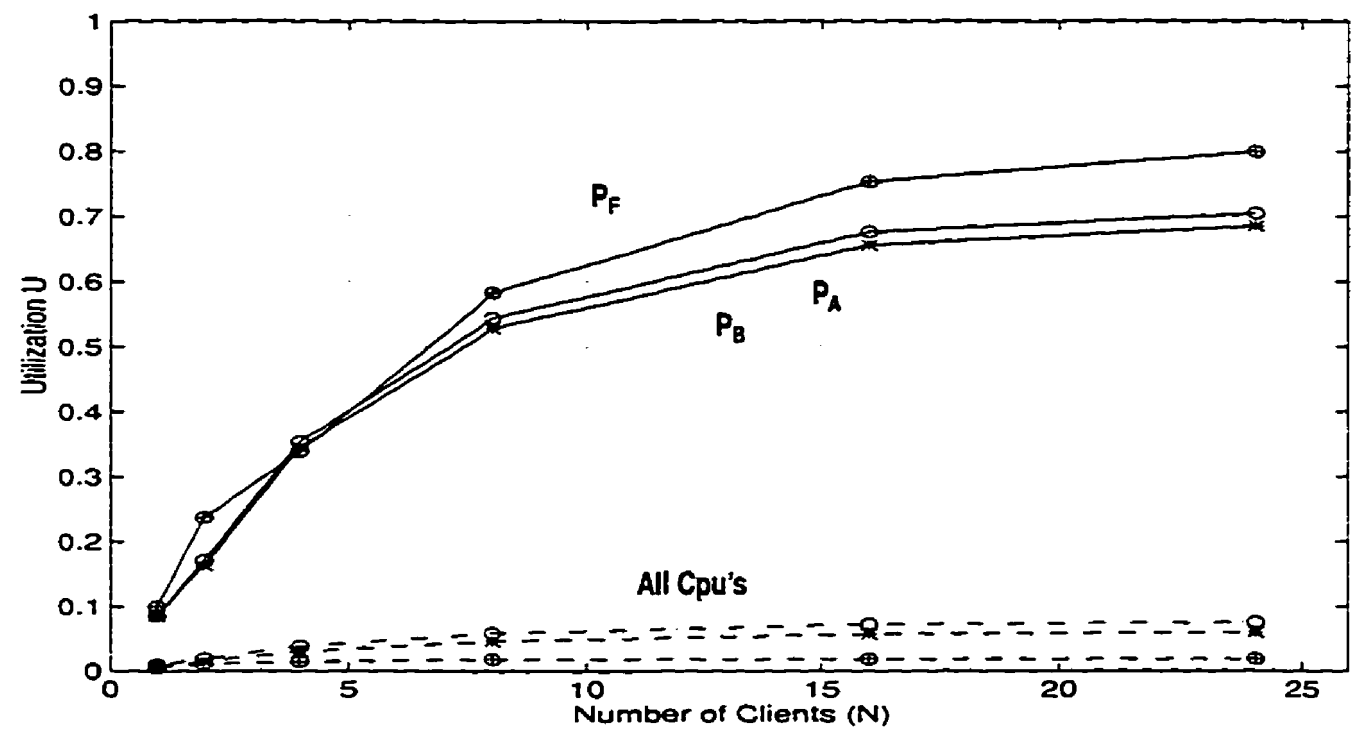

Figure A-28: Cpu \&-Process Utilizations of the F-ORB with $L=19200$ Bytes and 8 Cloning Degrees.

$P_{A}=$ Process Util. of Server $A, C P U_{A}=C p u$ Util of Server A.P.P $=$ Process Util. of f-agent.

Degree of Cloning $=8, D=200 \mathrm{~ms}, L=19200$ Bytes, $S_{A}=10 \mathrm{~ms}, S_{B}=15 \mathrm{~ms}$

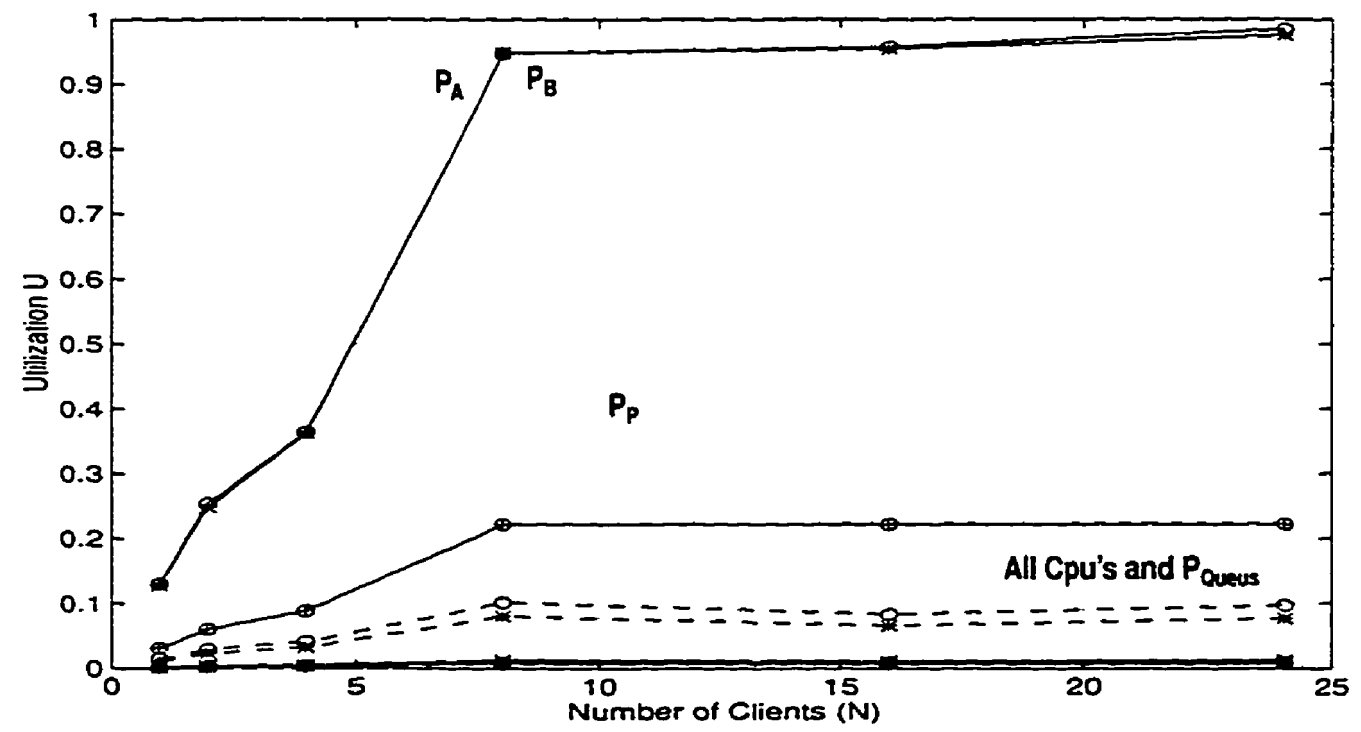

Figure A-29: Cpu \&Process Utilizations of the P-ORB with $L=19200$ Bytes and 8 Cloning Degrees.

$P_{A}=$ Process Util. of Server A. CPU ${ }_{A}=C p u$ Util of Server A.P $P_{P}=$ Process Util. of $p$-agent.

$P_{\text {Queves }}=$ Process Util. of dispatcher, Degree of Cloning $=8, D=200 \mathrm{~ms}, L=19200$ Bytes,

$\mathrm{S}_{\mathrm{A}}=10 \mathrm{~ms}, \mathrm{~S}_{\mathrm{B}}=15 \mathrm{~ms}$ 
Appendix B: Supplementary Results for

the Server Demand Set of

Experiments 


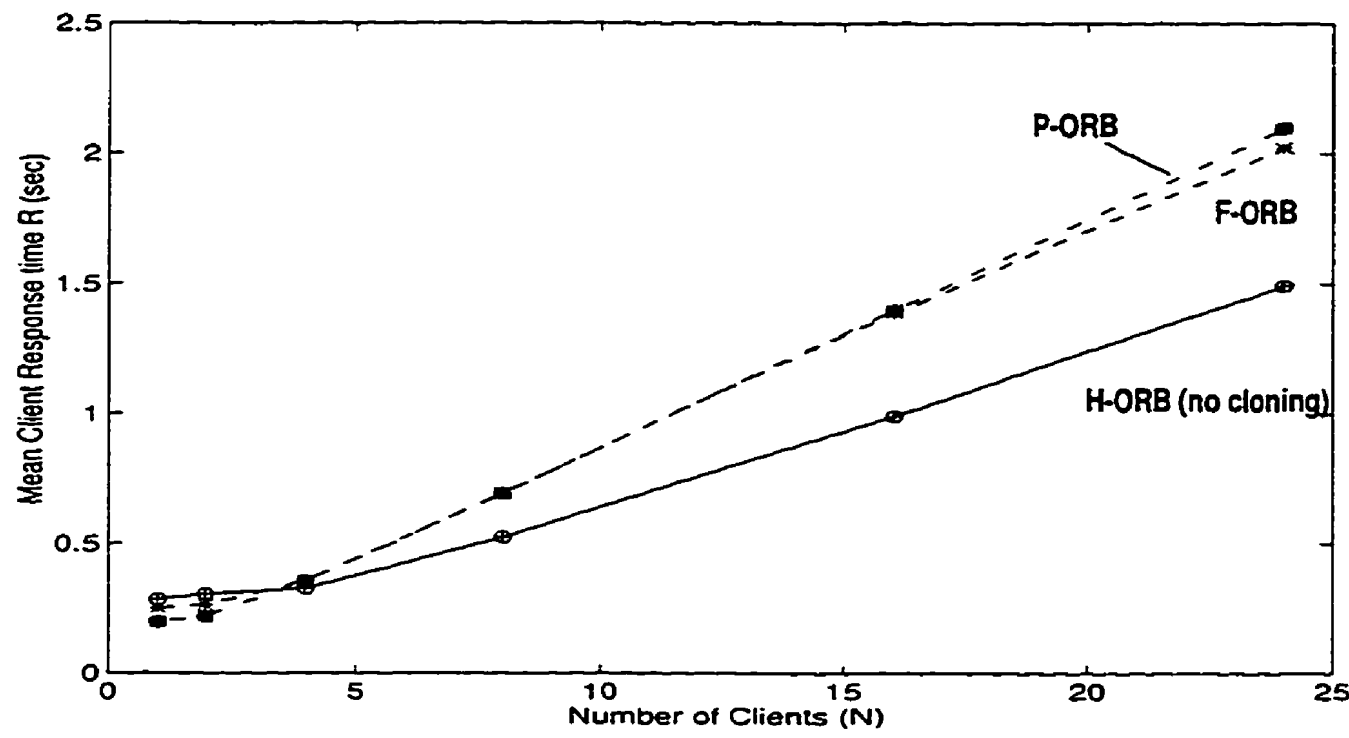

Figure B-1: The Response Time of the H-ORB, the F-ORB and the PORB with $S_{A} / S_{B}=50 / 75 m s$, and no Cloning.

Degree of Cloning $=1, D=10 \mathrm{~ms}, L=150$ Bytes, $S_{A}=50 \mathrm{~ms}, S_{B}=75 \mathrm{~ms}$

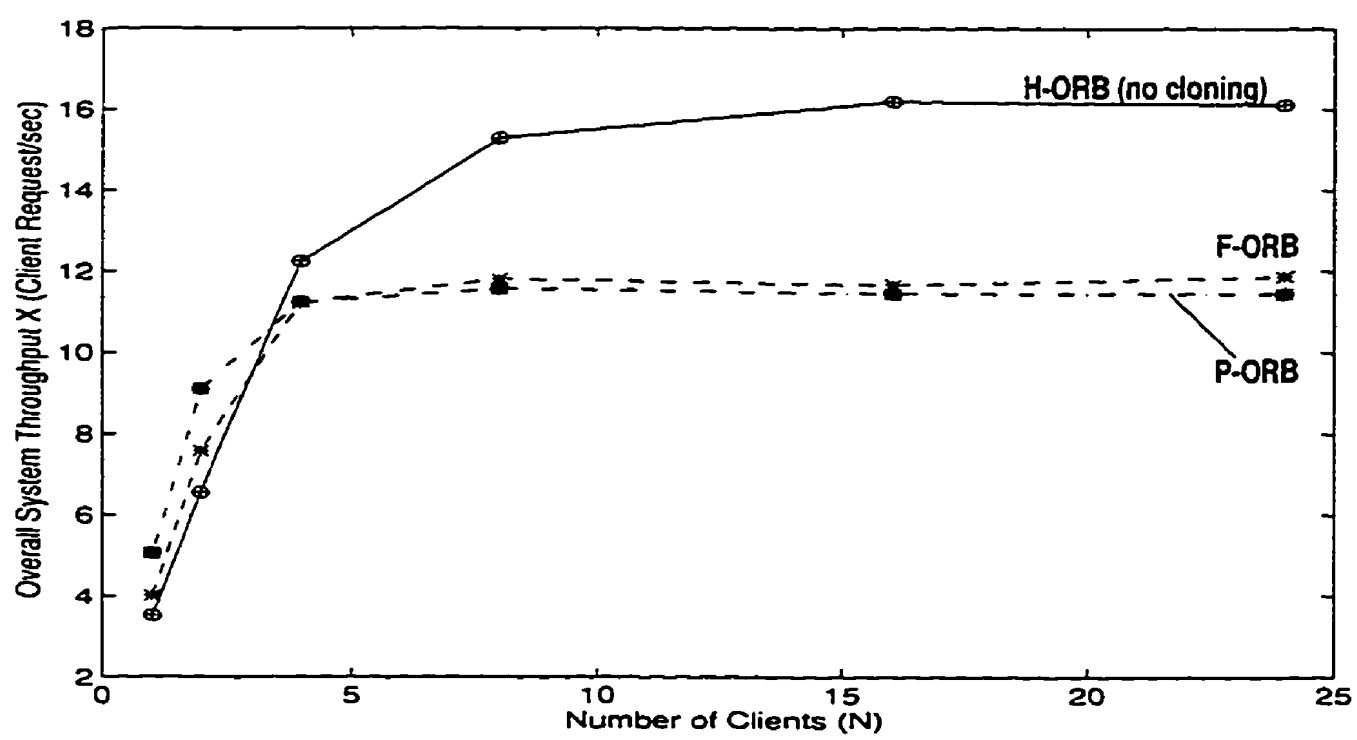

Figure B-2: The Throughput of the H-ORB, the F-ORB and the P-ORB with $S_{A} / S_{B}=50 / 75 m s$, and no Cloning.

Degree of Cloning $=1, D=10 \mathrm{~ms}, L=150$ Bytes, $S_{A}=50 \mathrm{~ms}, S_{B}=75 \mathrm{~ms}$ 


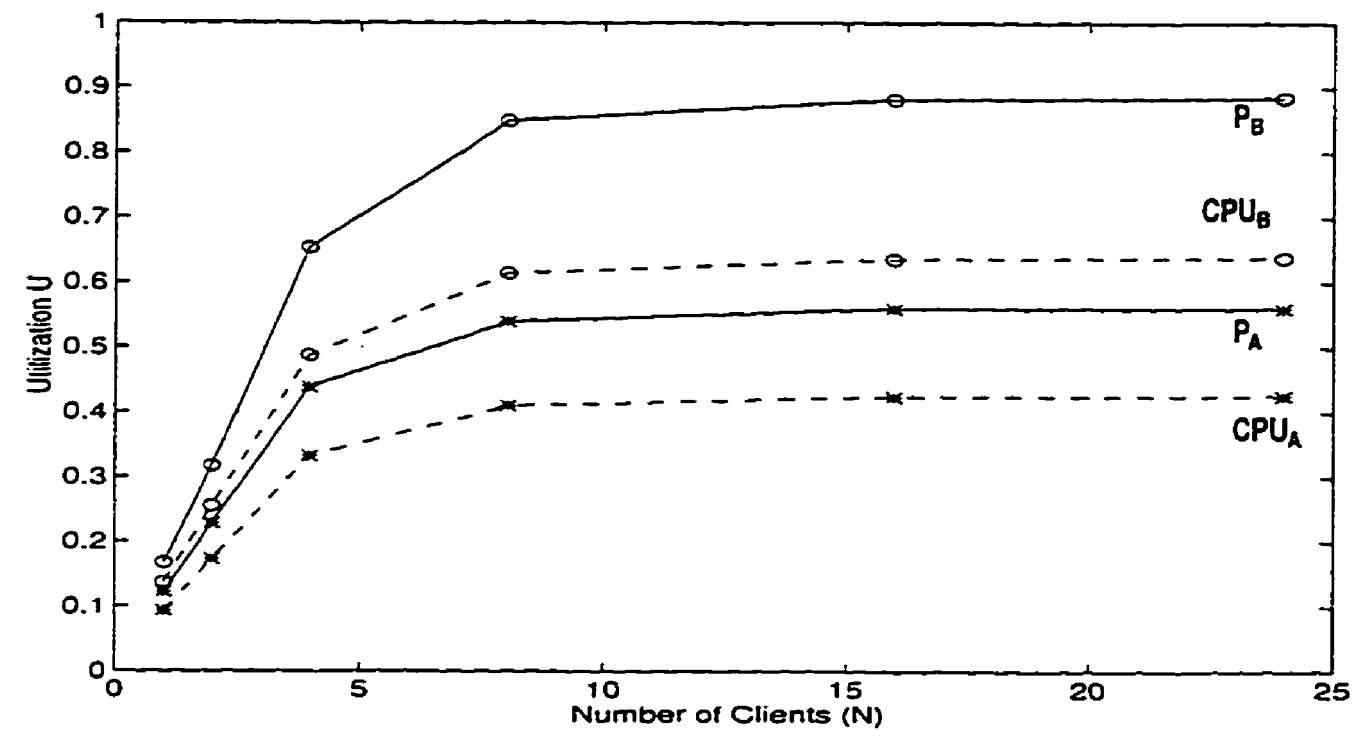

Figure B-3: Cpu and Process Utilizations of the H-ORB with $S_{A} / S_{B}=50 / 75 m s$, and no Cloning.

$P_{A}=$ Process Util. of Server A. CPU ${ }_{A}=$ Cpu Util of Server A.

Degree of Cloning $=1, D=10 \mathrm{~ms}, \hat{L}=150$ Bytes, $S_{A}=50 \mathrm{~ms}, S_{B}=75 \mathrm{~ms}$

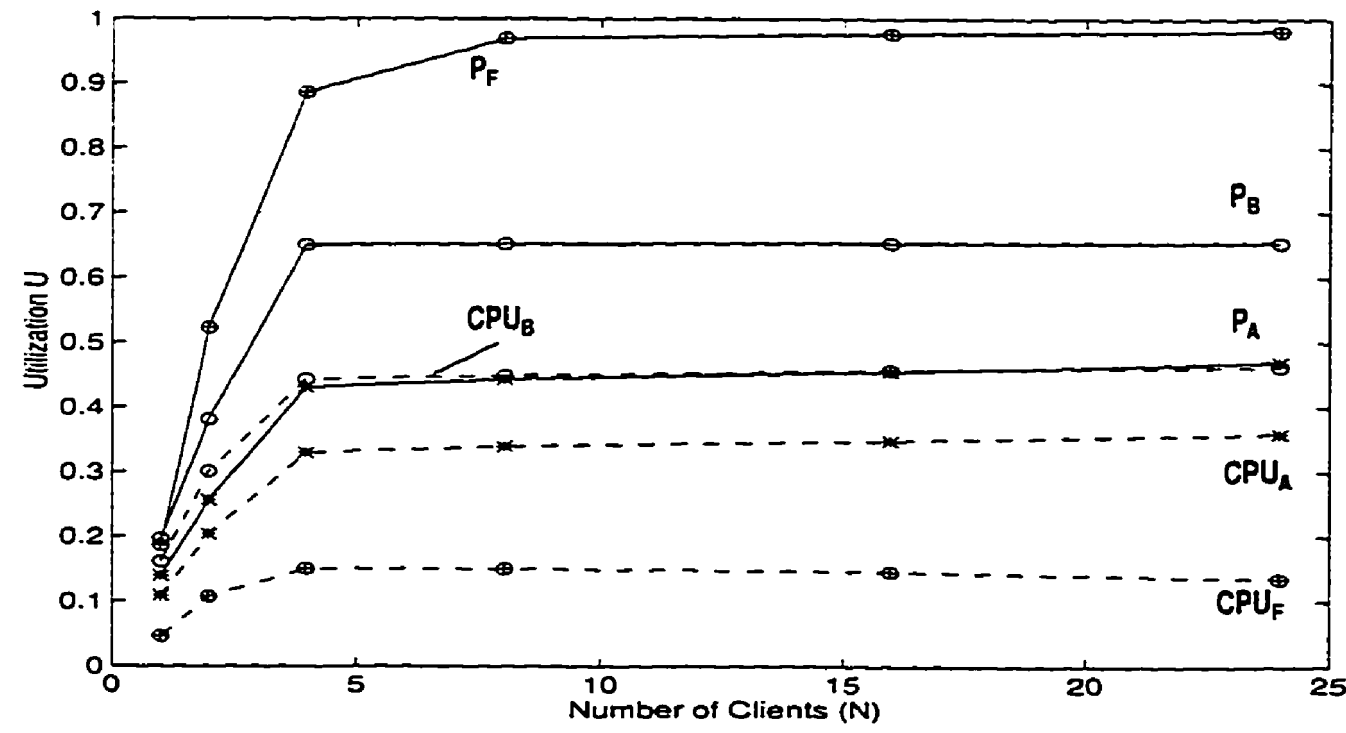

Figure B-4: Cpu \&Process Utilizations of the F-ORB with $S_{A} / S_{B}=50 / 75 m s$, and no Cloning.

$P_{A}=$ Process Util. of Server $A, C P U_{A}=C p u$ Util of Server $A, P_{F}=$ Process Util. of f-agent.

Degree of Cloning= I, D = $10 \mathrm{~ms}, \mathrm{~L}=150$ Bytes, $S_{A}=50 \mathrm{~ms}, S_{B}=75 \mathrm{~ms}$ 


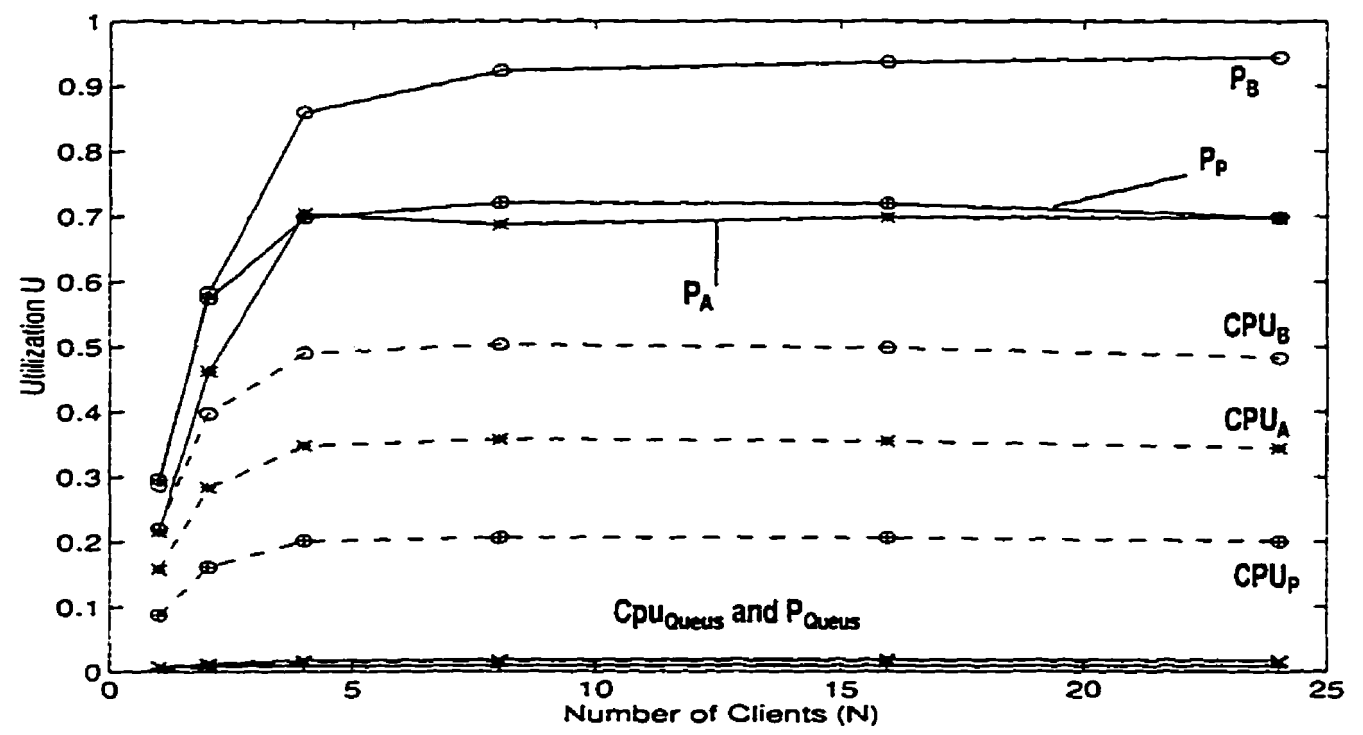

Figure B-5: Cpu \&Process Utilizations of the P-ORB with $S_{A} / S_{B}=50 / 75 m s$, and no Cloning.

$P_{A}=$ Process Util. of Server $A . C P U_{A}=C$ pu Util of Server $A, P_{P}=$ Process Util. of p-agent. $P_{\text {Queves }}=$ Process Util. of dispatcher, Degree of Cloning $=1, D=10 \mathrm{~ms}, L=150$ Bytes, $\mathrm{S}_{\mathrm{A}}=50 \mathrm{~ms}, \mathrm{~S}_{\mathrm{B}}=75 \mathrm{~ms}$ 


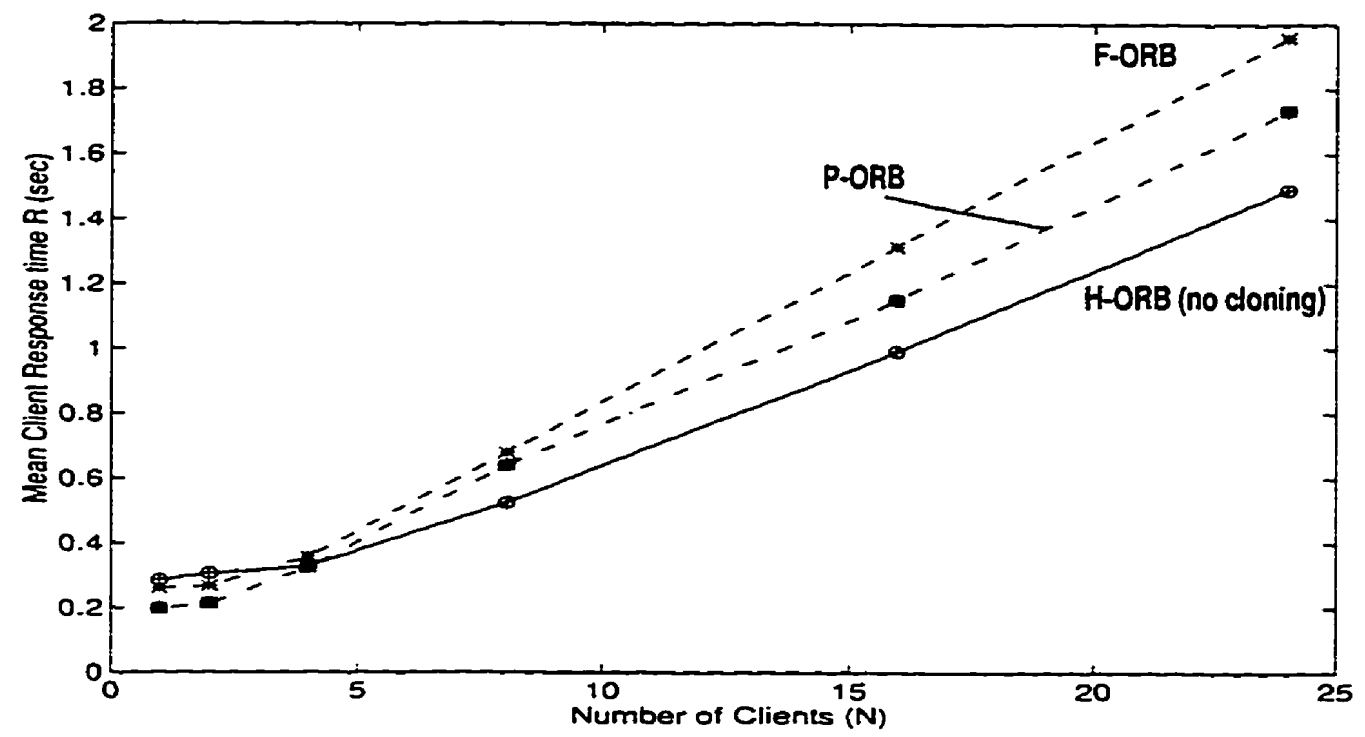

Figure B-6: Cpu and Process Utilizations of the H-ORB with $S_{A} / S_{B}=50 / 75 m s$, and 4 Cloning degrees.

$P_{A}=$ Process Util. of Server A, CPU $=C$ pu Util of Server $A$.

Degree of Cloning $=4, D=10 \mathrm{~ms}, \mathrm{~L}=150$ Bytes, $S_{A}=50 \mathrm{~ms}, S_{B}=75 \mathrm{~ms}$

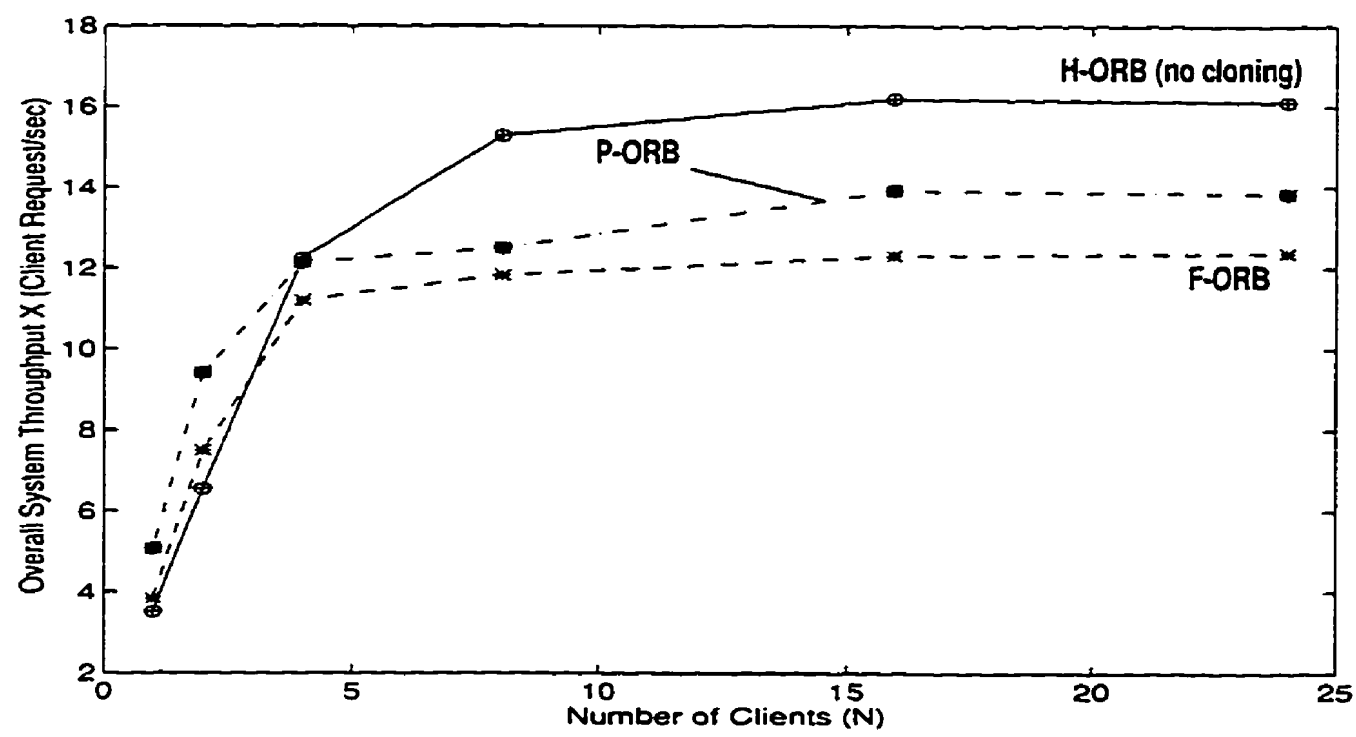

Figure B-7: The Throughput of the H-ORB, the F-ORB and the P-ORB with $S_{A} / S_{B}=50 / 75 m s$, and 4 Cloning degrees.

Degree of Cloning $=4, D=10 \mathrm{~ms}, L=150$ Bytes, $S_{A}=50 \mathrm{~ms}, S_{B}=75 \mathrm{~ms}$ 


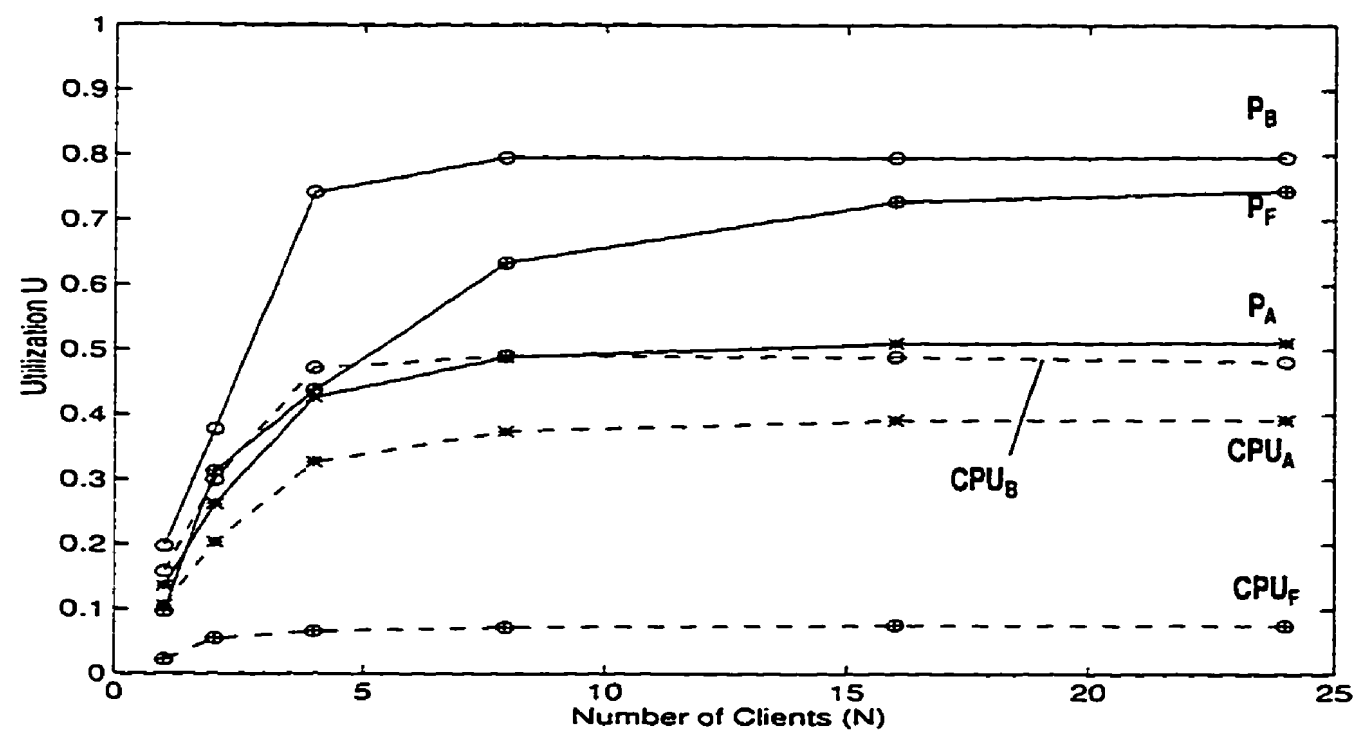

Figure B-8: Cpu \& Process Utilizations of the F-ORB with $S_{A} / S_{B}=50 / 75 m s$, and 4 Cloning degrees.

$P_{A}=$ Process Util. of Server $A, C P U_{A}=C$ pu Util of Server $A, P_{F}=$ Process Util. of f-agent. Degree of Cloning $=4, D=10 \mathrm{~ms}, \mathrm{~L}=150$ Bytes, $S_{A}=50 \mathrm{~ms}, S_{B}=75 \mathrm{~ms}$

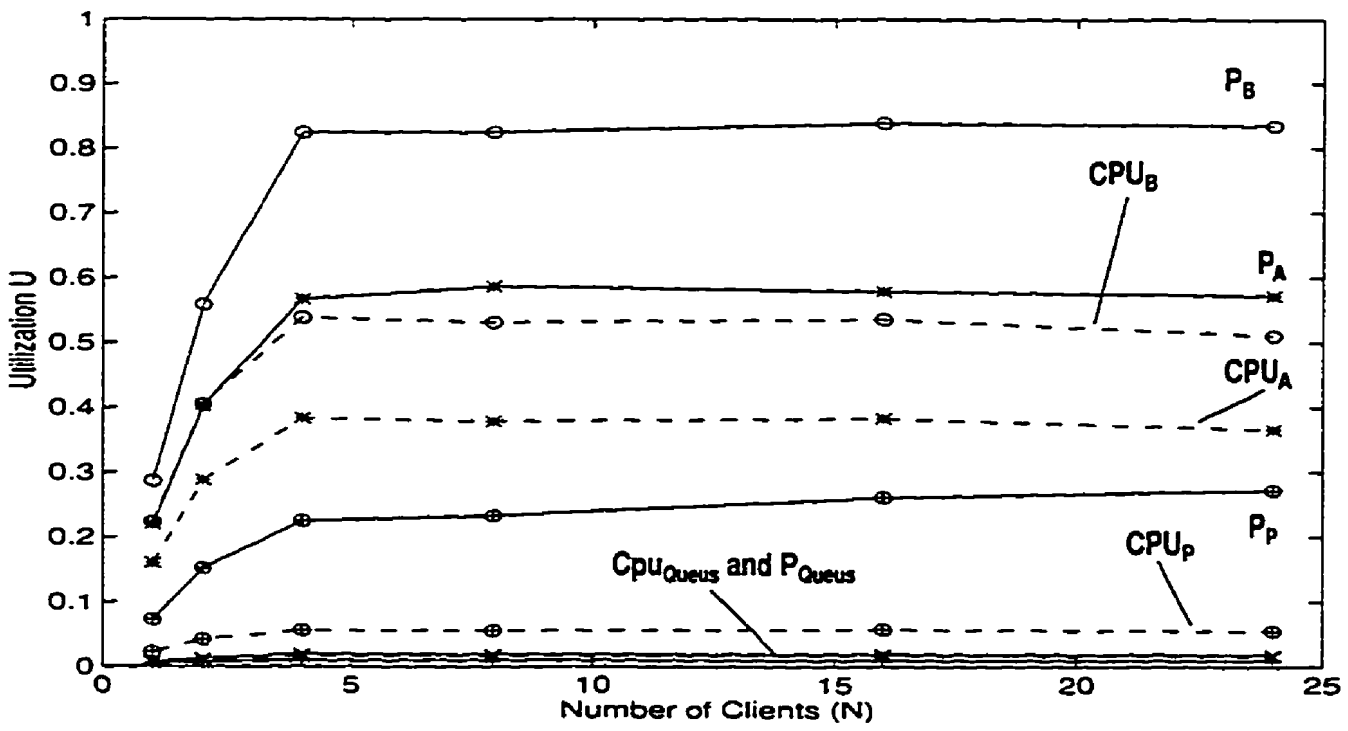

Figure B-9: Cpu \&Process Utilizations of the P-ORB with $S_{A} / S_{B}=50 / 75 m s$, and 4 Cloning degrees.

$P_{A}=$ Process Util. of Server $A, C P U_{A}=C p u$ Util of Server $A, P_{P}=$ Process Util. of p-agent. $P_{Q u e v e s}=$ Process Util. of dispatcher, Degree of Cloning $=4, D=10 \mathrm{~ms}, L=150$ Bytes,

$S_{A}=50 \mathrm{~ms}, S_{B}=75 \mathrm{~ms}$ 


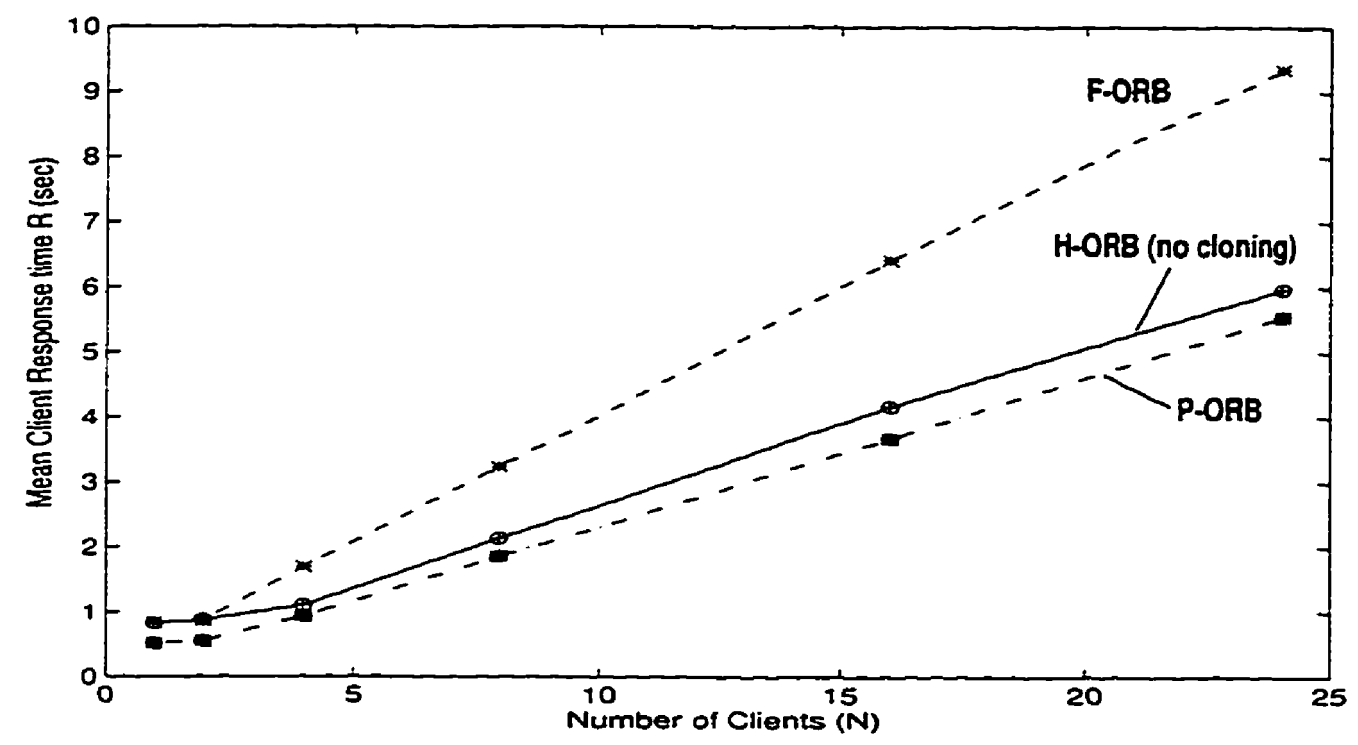

Figure B-10: The Response Time of the H-ORB, the F-ORB and the PORB with $S_{A} / S_{B}=250 / 375 m s$, and 4 Cloning degrees.

Degree of Cloning $=4, D=10 \mathrm{~ms}, L=150$ Bytes, $S_{A}=250 \mathrm{~ms}, S_{B}=375 \mathrm{~ms}$

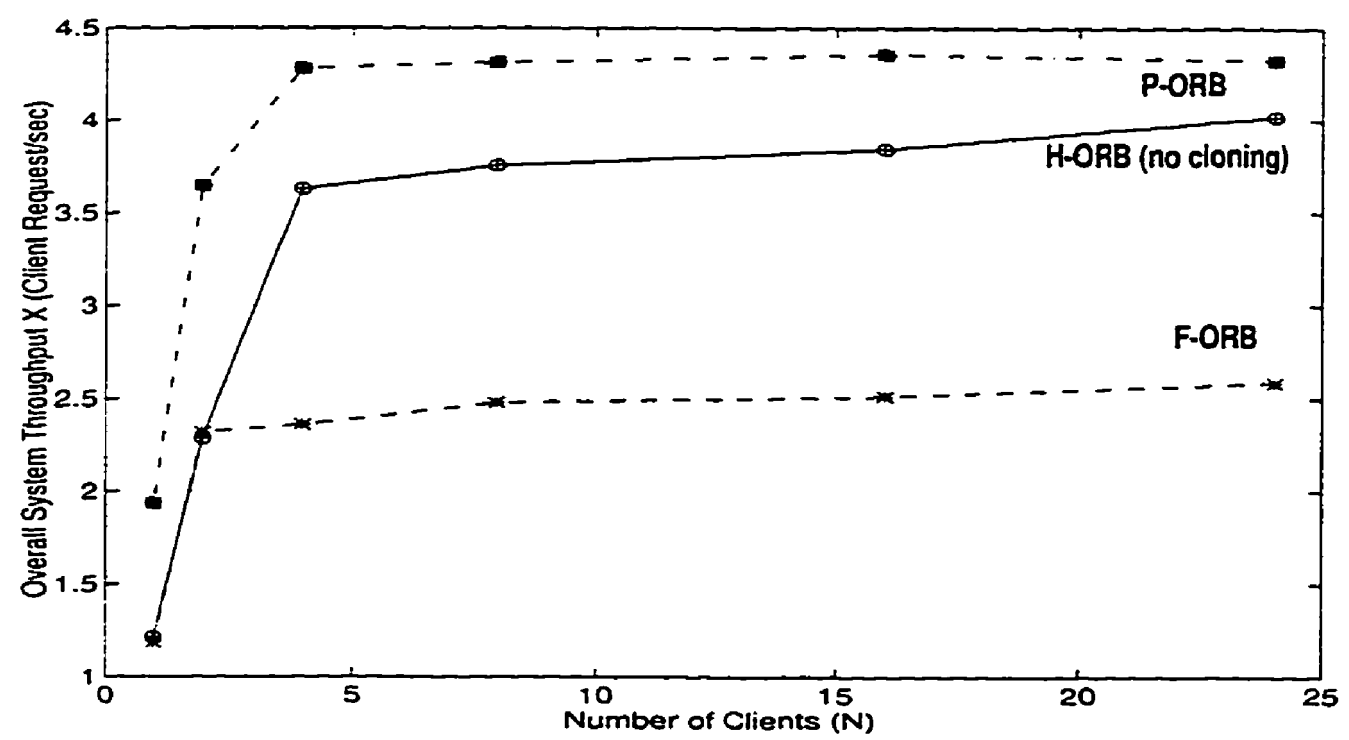

Figure B-11: The Throughput of the H-ORB, the F-ORB and the P-ORB with $S_{A} / S_{B}=250 / 375 m s$, and 4 Cloning degrees.

Degree of Cloning $=4, D=10 \mathrm{~ms}, L=150$ Byres, $S_{A}=250 \mathrm{~ms}, S_{B}=375 \mathrm{~ms}$ 


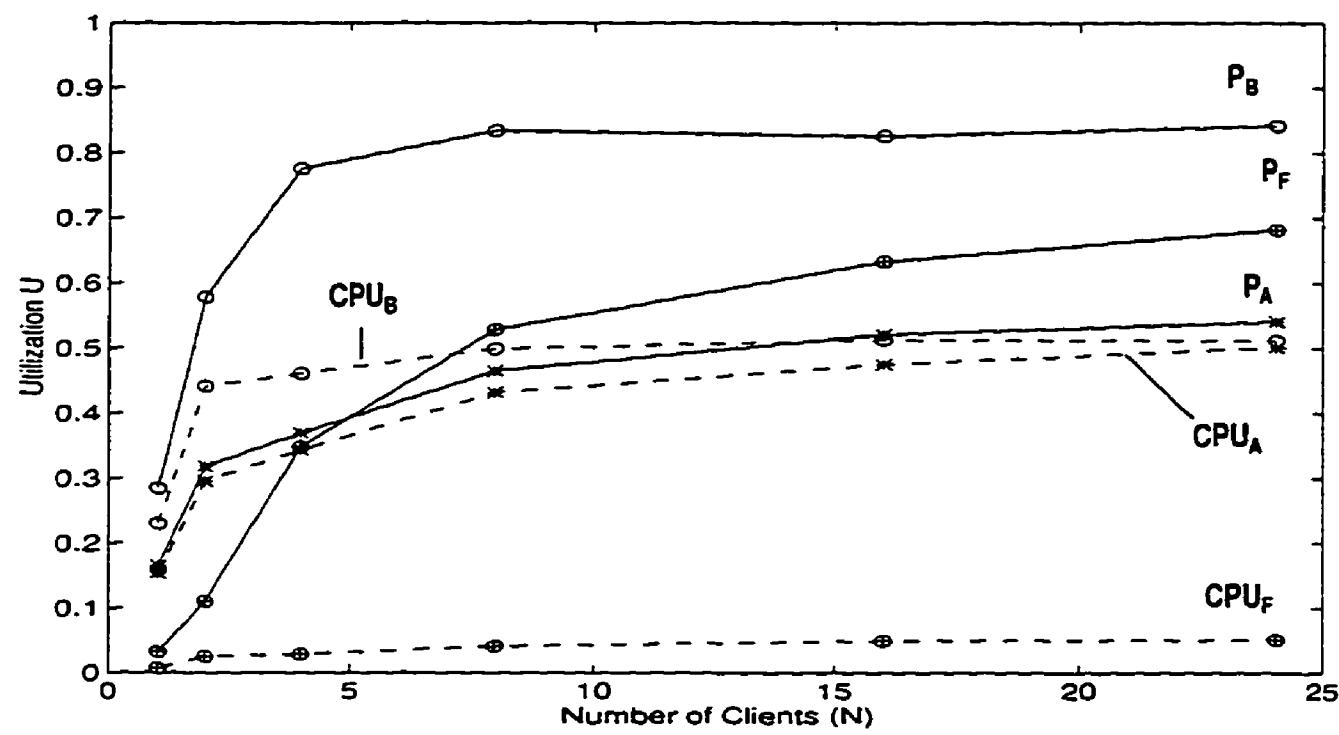

Figure B-12: Cpu \&Process Utilizations of the F-ORB with $S_{A} / S_{B}=250 / 375 m s$, and 4 Cloning Degrees.

$P_{A}=$ Process Util. of Server $A, C P U_{A}=C p u$ Util of Server $A, P_{F}=$ Process Util. of f-agent.

Degree of Cloning $=4, D=10 \mathrm{~ms}, L=150$ Bytes, $S_{A}=250 \mathrm{~ms}, S_{B}=375 \mathrm{~ms}$

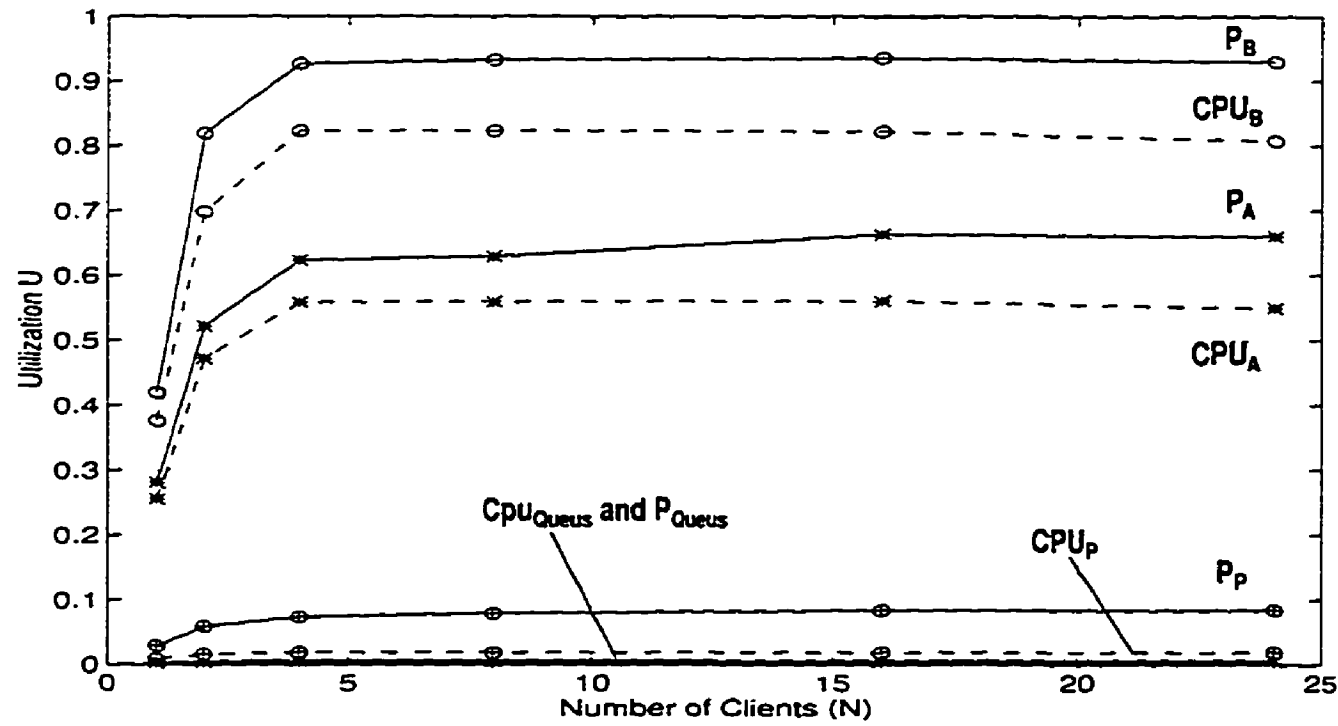

Figure B-13: Cpu \&Process Utilizations of the $P$-ORB with $S_{A} / S_{B}=250 / 375 m s$, and 4 Cloning Degrees.

$P_{A}=$ Process Util. of Server $A, C P U_{A}=C p u$ Util of Server $A, P_{P}=$ Process Util. of p-agent.

$P_{\text {Queues }}=$ Process Util. of dispatcher, Degree of Cloning $=4, D=10 \mathrm{~ms}, L=150$ Bytes,

$\mathrm{S}_{\mathrm{A}}=250 \mathrm{~ms}, \mathrm{~S}_{\mathrm{B}}=375 \mathrm{~ms}$ 


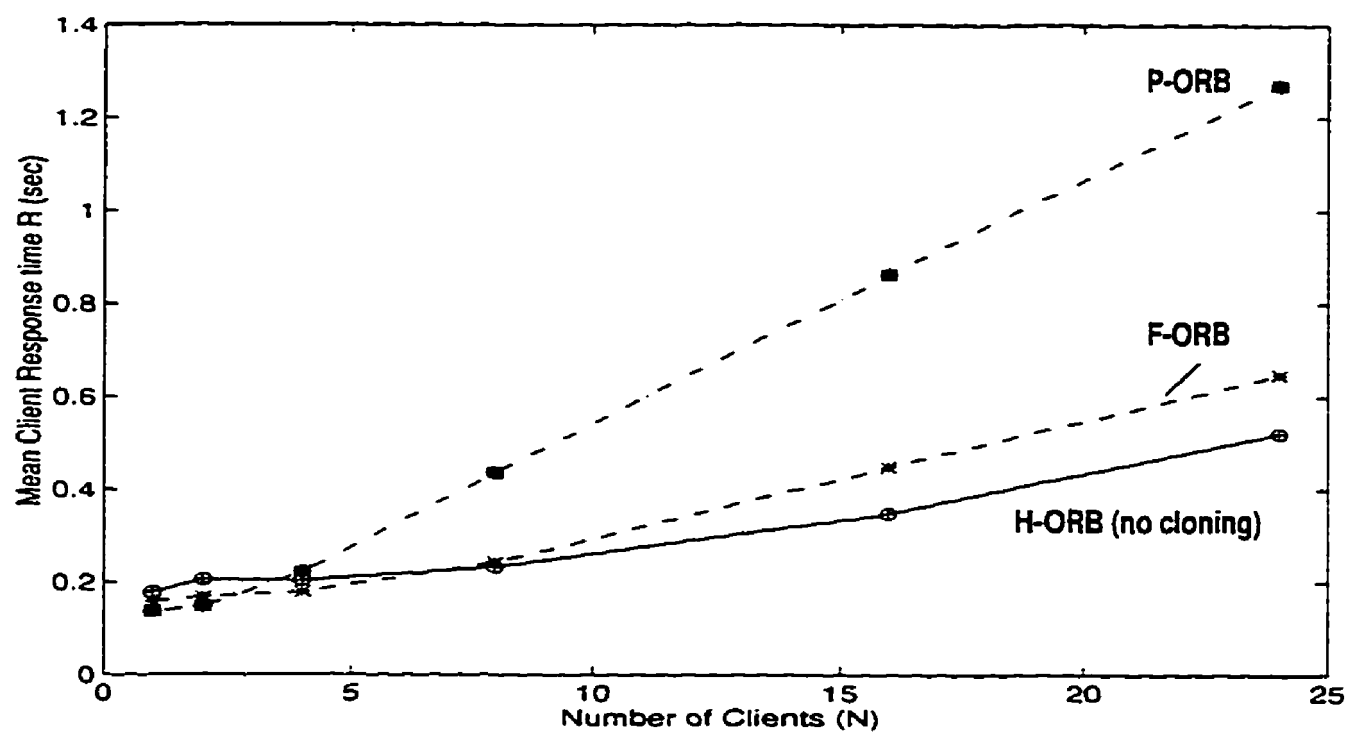

Figure B-14: The Response Time of the H-ORB, the F-ORB and the PORB with $S_{A} / S_{B}=10 / 15 m s$, and 8 Cloning Degrees.

Degree of Cloning $=8, D=10 \mathrm{~ms}, L=150$ Bytes, $S_{A}=10 \mathrm{~ms}, S_{B}=15 \mathrm{~ms}$

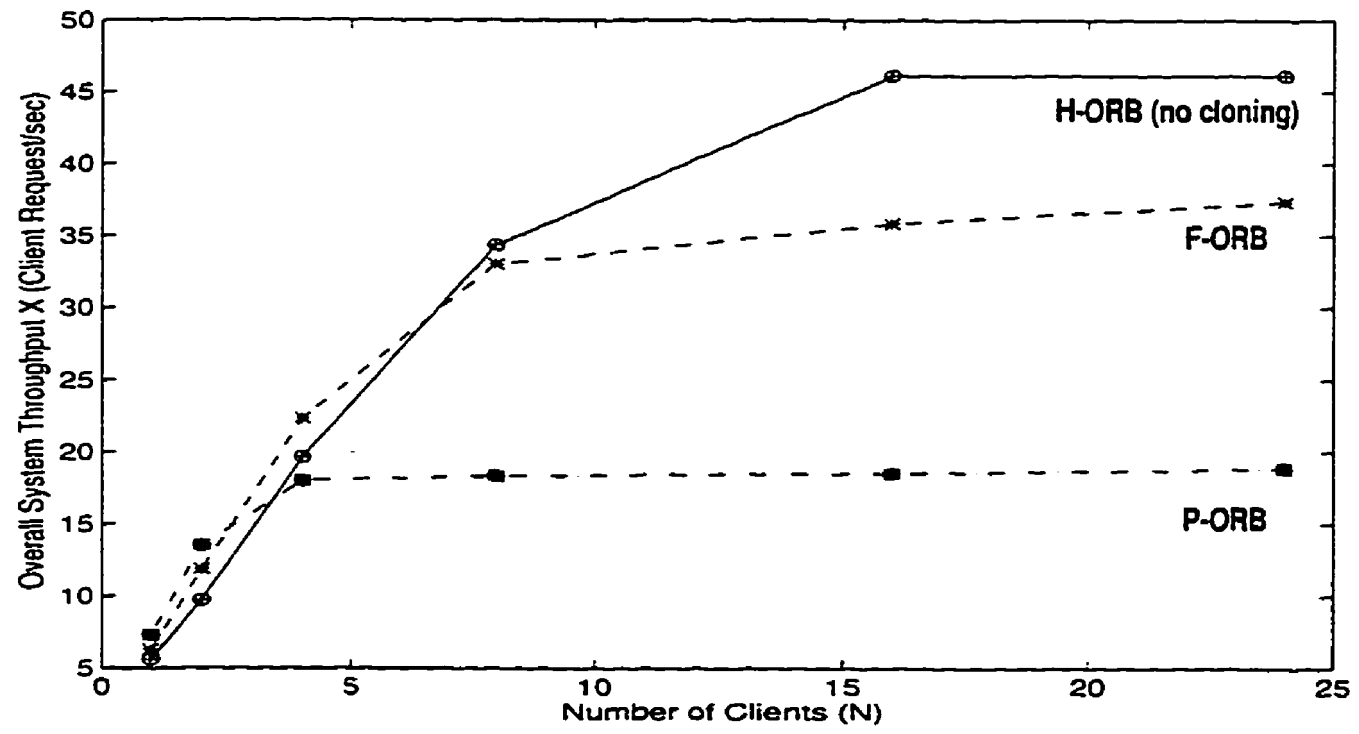

Figure B-15: The Throughput of the H-ORB, the F-ORB and the P-ORB with $S_{A} / S_{B}=10 / 15 m s$, and 8 Cloning Degrees.

Degree of Cloning $=8, D=10 \mathrm{~ms}, L=150$ Bytes, $S_{A}=10 \mathrm{~ms}, S_{B}=15 \mathrm{~ms}$ 


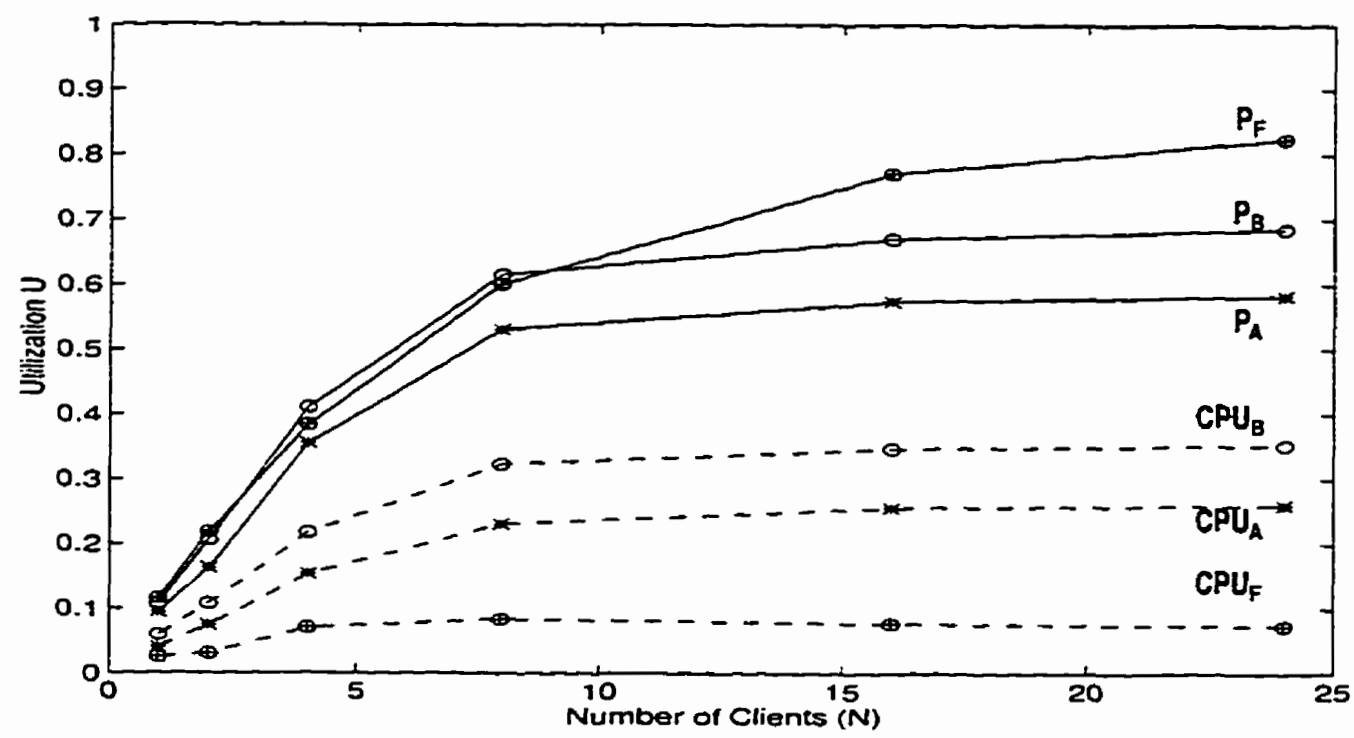

Figure B-16: Cpu \&Process Utilizations of the F-ORB with $S_{A} / S_{B}=10 / 15 m s$, and 8 Cloning Degrees.

$P_{A}=$ Process Util. of Server $A, C P U_{A}=C p u$ Util of Server A,P $=$ Process Uril. of f-agent.

Degree of Cloning $=8, D=10 \mathrm{~ms}, \mathrm{~L}=150$ Bytes, $S_{A}=10 \mathrm{~ms}, S_{B}=15 \mathrm{~ms}$

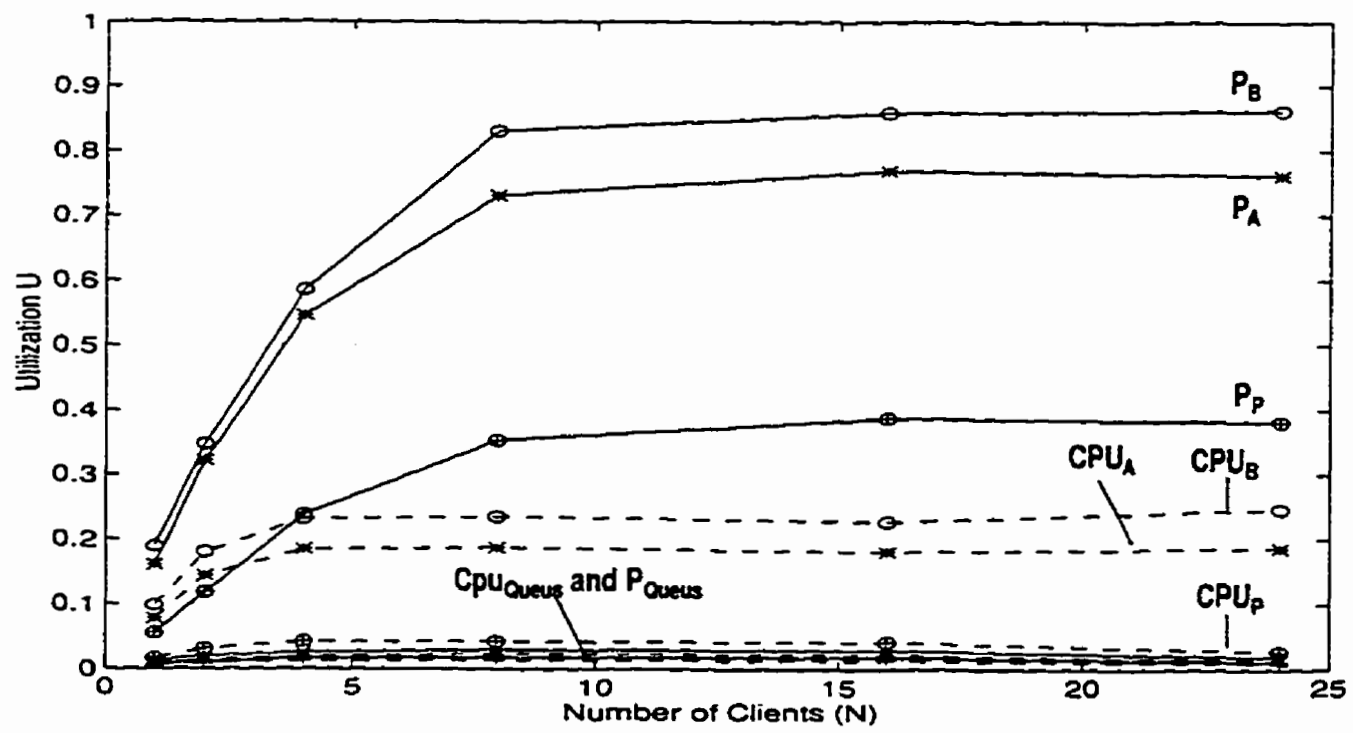

Figure B-17: Cpu \&Process Utilizations of the P-ORB with $S_{A} / S_{B}=10 / 15 m s$, and 8 Cloning Degrees.

$\mathrm{P}_{\mathrm{A}}=$ Process Util. of Server $\mathrm{A}, C P \mathrm{U}_{\mathrm{A}}=\mathrm{C}_{\mathrm{pu}}$ Util of Server $\mathrm{A}, \mathrm{P}_{\mathrm{P}}=$ Process Util. of p-agent.

$P_{Q u e u e s}=$ Process Util. of dispatcher, Degree of Cloning $=8, D=10 \mathrm{~ms}, L=150$ Bytes,

$S_{A}=10 \mathrm{~ms}, S_{B}=15 \mathrm{~ms}$ 


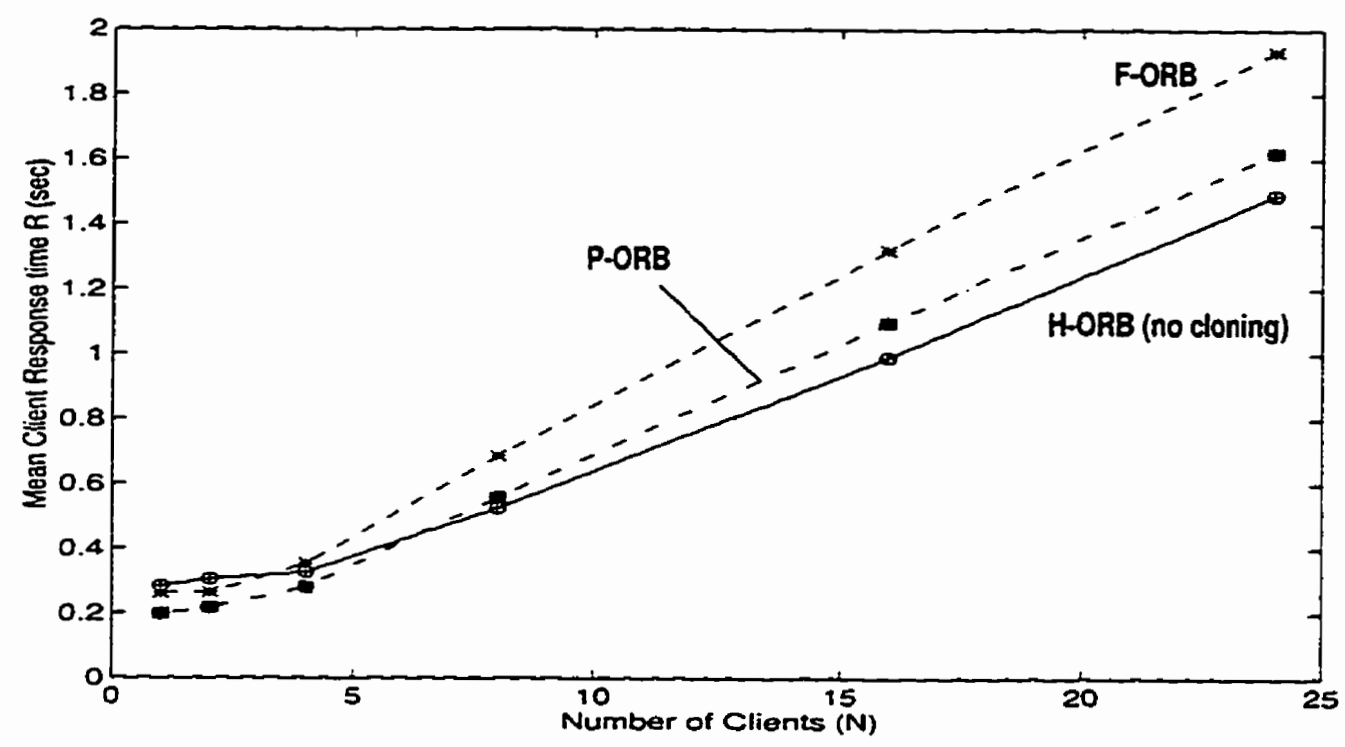

Figure B-18: The Response Time of the H-ORB, the F-ORB and the P$O R B$ with $S_{A} / S_{B}=50 / 75 m s$, and 8 Cloning degrees.

Degree of Cloning $=8, D=10 \mathrm{~ms}, L=150$ Bytes, $S_{A}=50 \mathrm{~ms}, S_{B}=75 \mathrm{~ms}$

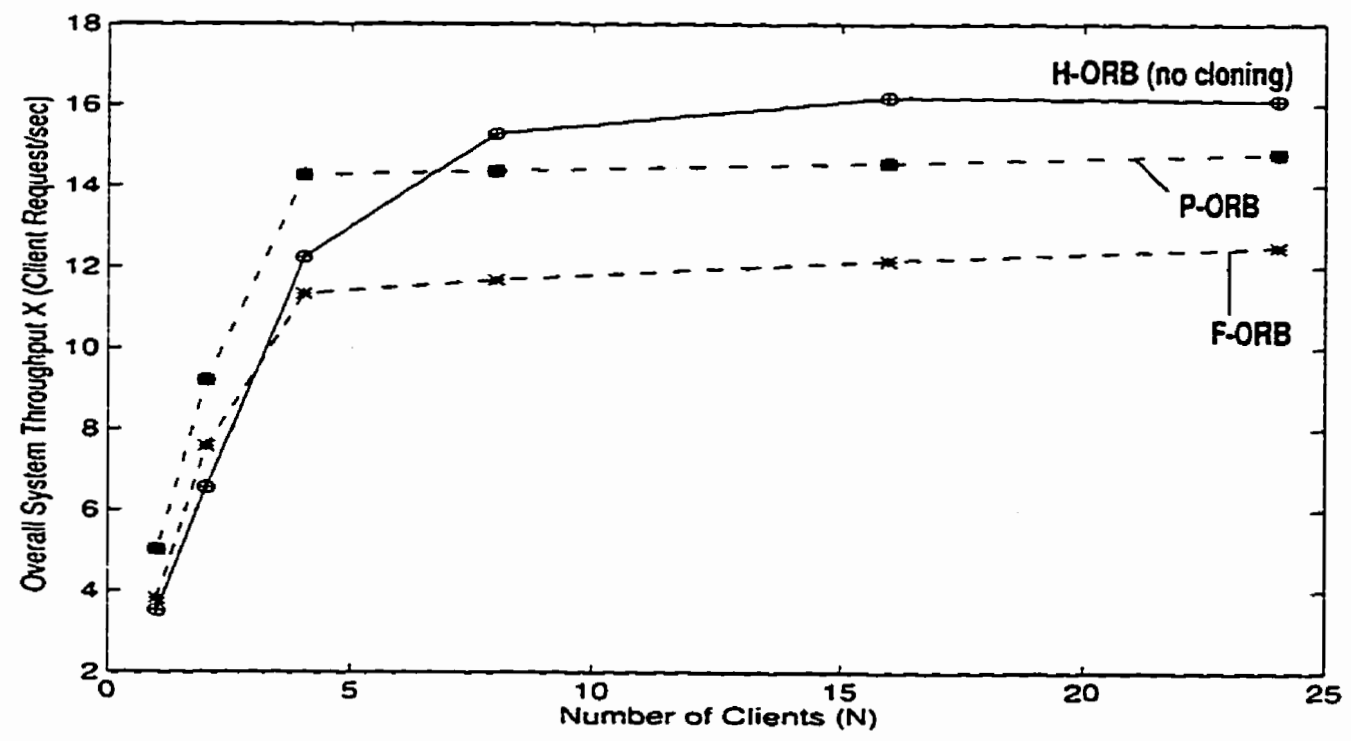

Figure B-19: The Throughput of the H-ORB, the F-ORB and the P-ORB with $S_{A} / S_{B}=50 / 75 m s$, and 8 Cloning degrees.

Degree of Cloning $=8, D=10 \mathrm{~ms}, L=150$ Bytes, $S_{A}=50 \mathrm{~ms}, S_{B}=75 \mathrm{~ms}$ 


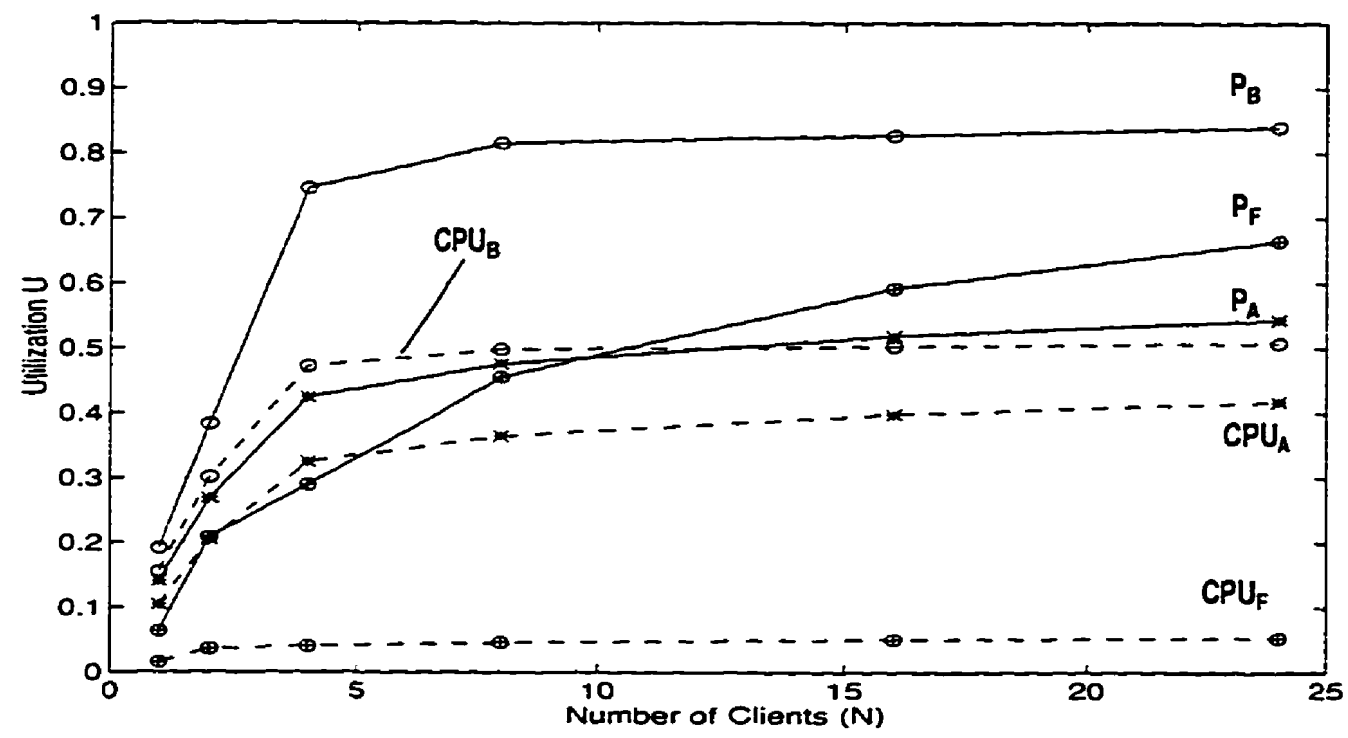

Figure B-20: Cpu \&Process Utilizations of the F-ORB with $S_{A} / S_{B}=50 / 75 m s$, and 8 Cloning degrees.

$P_{A}=$ Process Util. of Server $A, C P U_{A}=C p u$ Util of Server $A, P_{F}=$ Process Util. of f-agent. Degree of Cloning $=8, D=10 \mathrm{~ms}, \mathrm{~L}=150$ Bytes, $S_{A}=50 \mathrm{~ms}, S_{B}=75 \mathrm{~ms}$

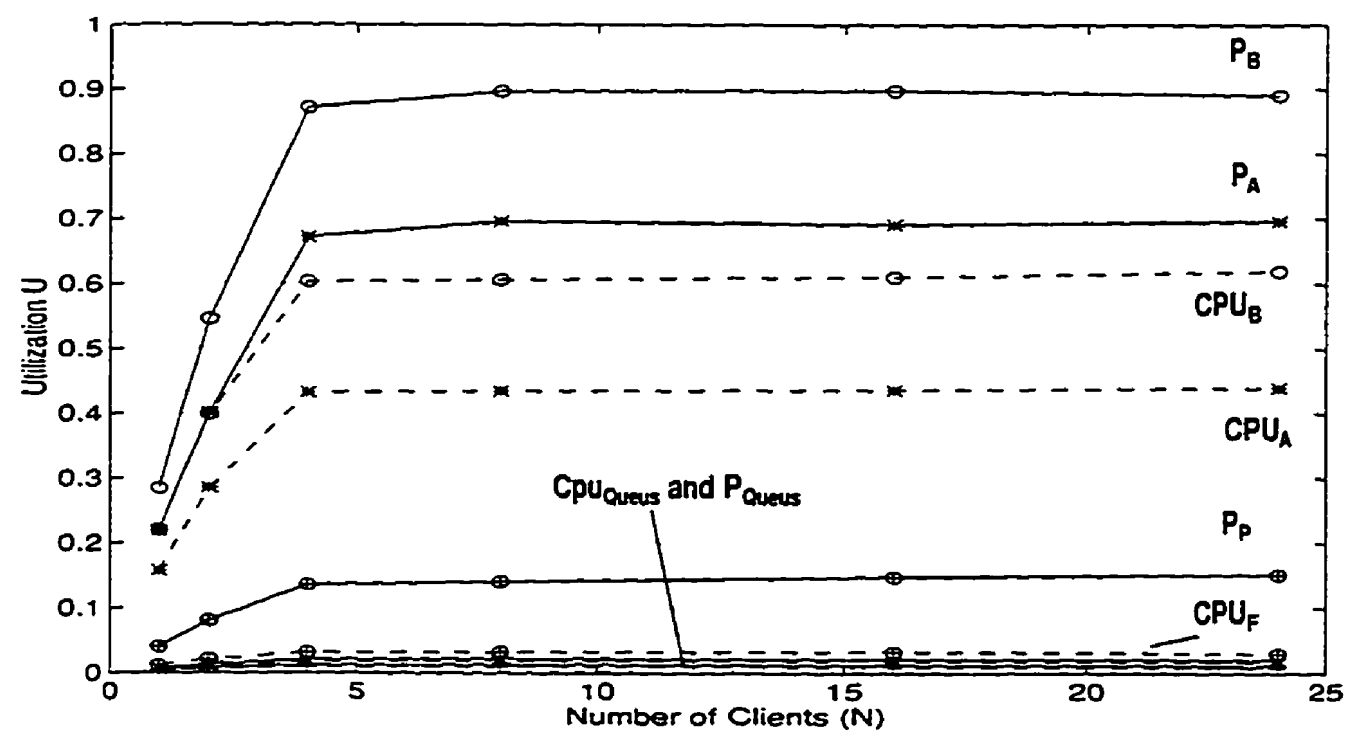

Figure B-21: Cpu \&Process Utilizations of the $P-O R B$ with $S_{A} / S_{B}=50 / 75 m s$, and 8 Cloning degrees.

$\mathrm{P}_{\mathrm{A}}=$ Process Util. of Server A, $C P U_{A}=C p u$ Util of Server $A, P_{P}=$ Process Util. of p-agent. $P_{Q u e u e s}=$ Process Util. of dispatcher, Degree of Cloning $=8, D=10 \mathrm{~ms}, \mathrm{~L}=150$ Bytes, $\mathrm{S}_{\mathrm{A}}=50 \mathrm{~ms}, \mathrm{~S}_{\mathrm{B}}=75 \mathrm{~ms}$ 


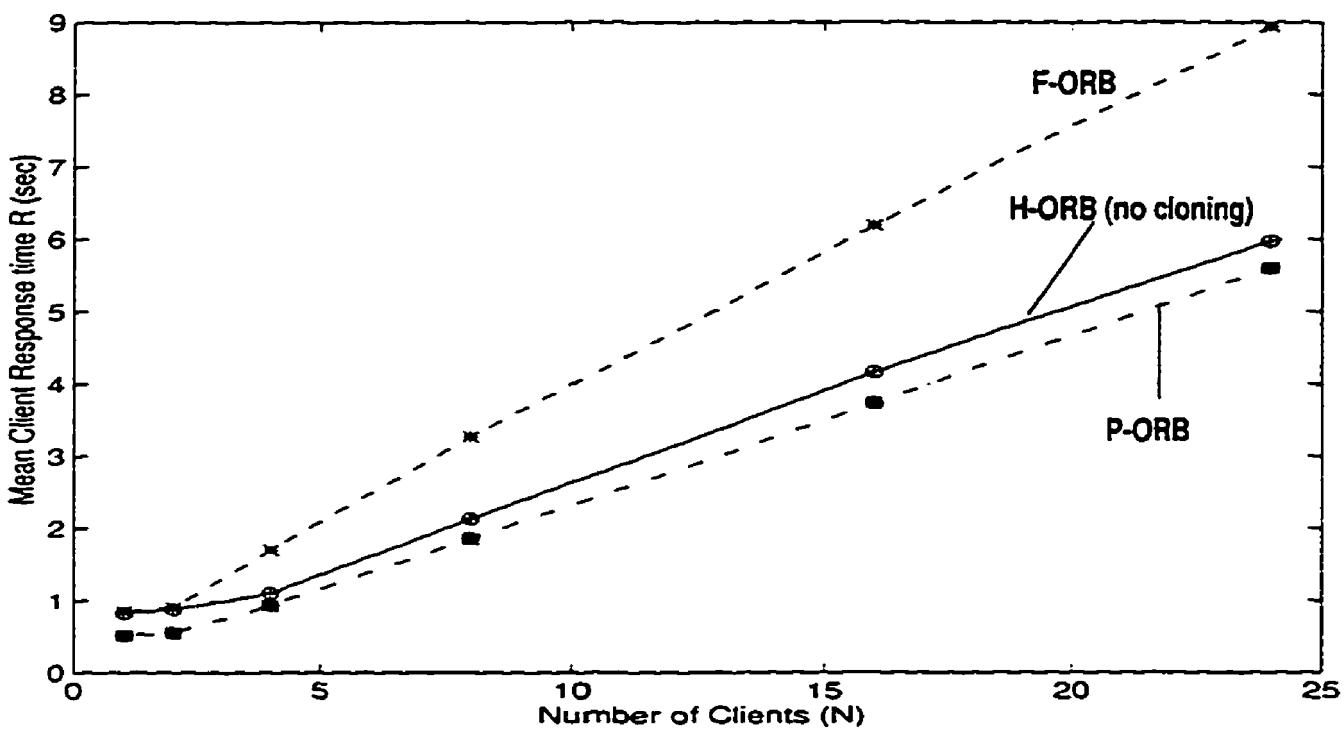

Figure B-22: The Response Time of the H-ORB, the F-ORB and the PORB with $S_{A} / S_{B}=250 / 375 m s$, and 8 Cloning degrees.

Degree of Cloning $=8, D=10 \mathrm{~ms}, L=150$ Bytes, $S_{A}=250 \mathrm{~ms}, S_{B}=375 \mathrm{~ms}$

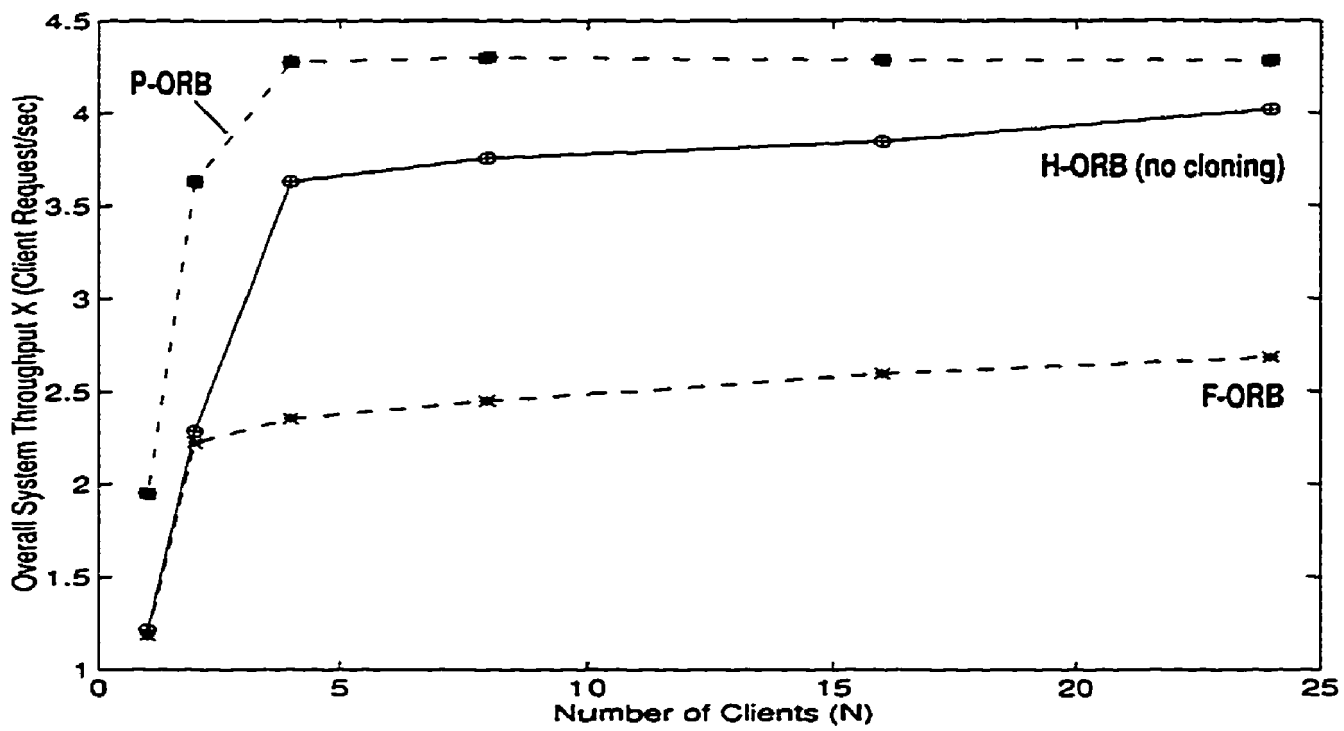

Figure B-23: The Throughput of the H-ORB, the F-ORB and the P-ORB with $S_{A} / S_{B}=250 / 375 m s$, and 8 Cloning degrees.

Degree of Cloning $=8, D=10 \mathrm{~ms}, L=150$ Bytes, $S_{A}=250 \mathrm{~ms}, S_{B}=375 \mathrm{~ms}$ 


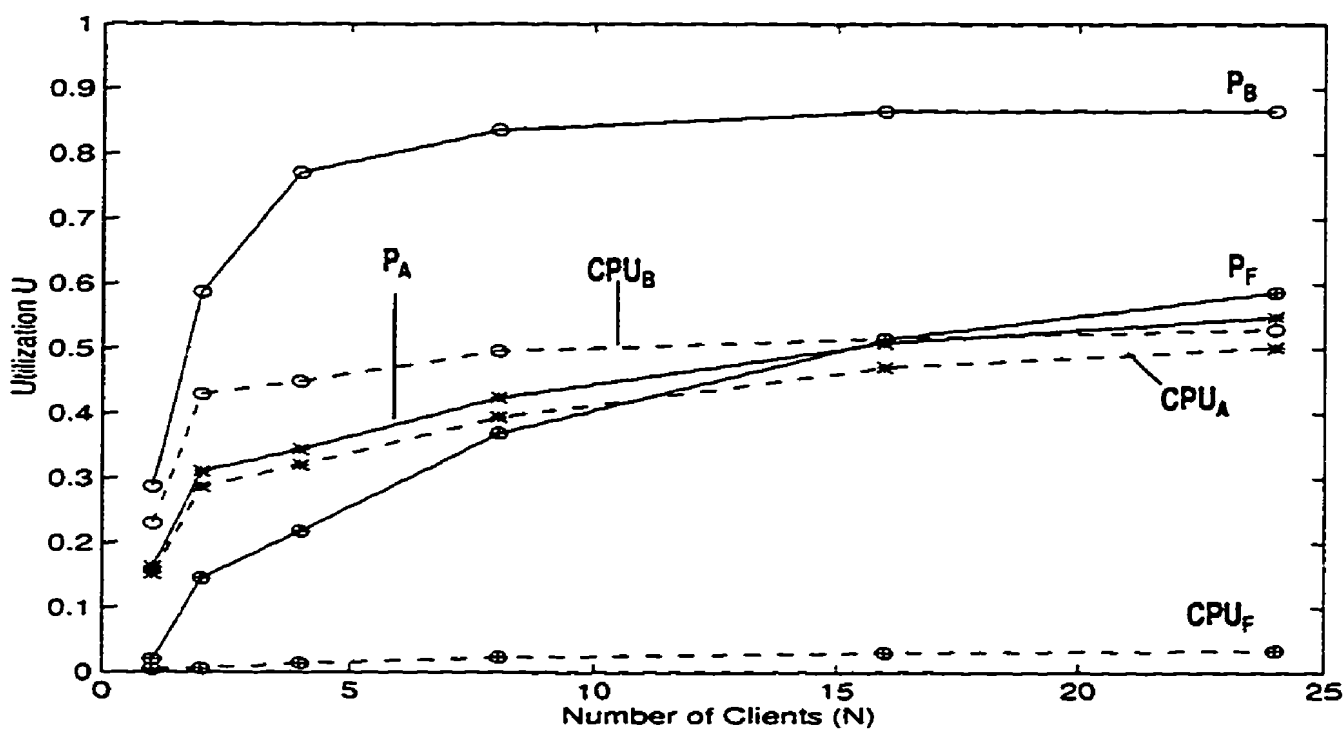

Figure B-24: Cpu \&Process Utilizations of the F-ORB with $S_{A} / S_{B}=250 / 375 m s$, and 8 Cloning degrees.

$P_{A}=$ Process Util. of Server $A, C P U_{A}=C$ pu Util of Server $A, P_{F}=$ Process Util. of f-agent.

Degree of Cloning $=8, D=10 \mathrm{~ms}, \mathrm{~L}=150$ Bytes, $S_{A}=250 \mathrm{~ms}, S_{B}=375 \mathrm{~ms}$

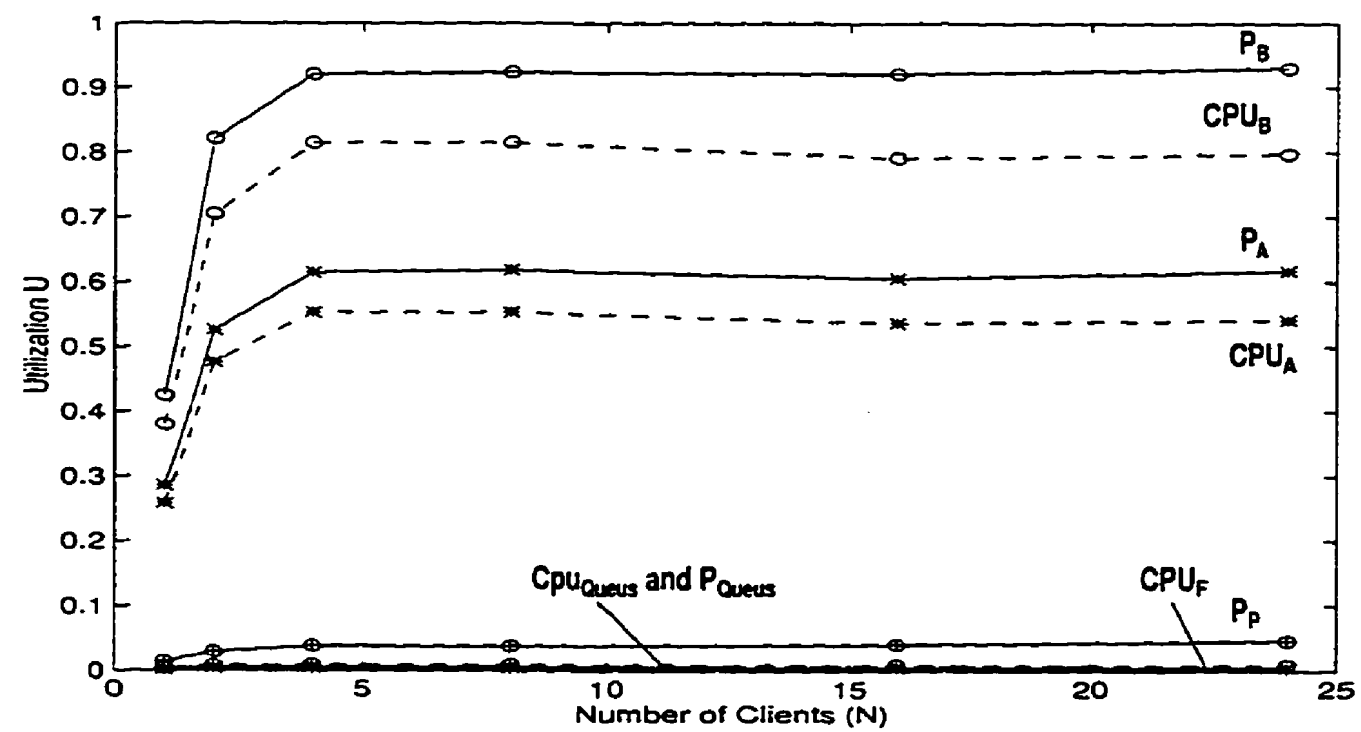

Figure B-25: Cpu \& Process Utilizations of the $P-O R B$ with $S_{A} / S_{B}=250 / 375 m s$, and 8 Cloning degrees.

$\mathrm{P}_{\mathrm{A}}=$ Process Util. of Server $\mathrm{A}, C P U_{\mathrm{A}}=\mathrm{Cpu}$ Util of Server $\mathrm{A}, \mathrm{P}_{\mathrm{P}}=$ Process Util. of p-agent. $P_{Q u e v e s}=$ Process Util. of dispatcher, Degree of Cloning $=8, D=10 \mathrm{~ms}, \mathrm{~L}=150$ Bytes,

$S_{A}=250 \mathrm{~ms}, S_{B}=375 \mathrm{~ms}$ 
Appendix C: Supplementary Results for the Inter-Node Delay Set of

\section{Experiments}




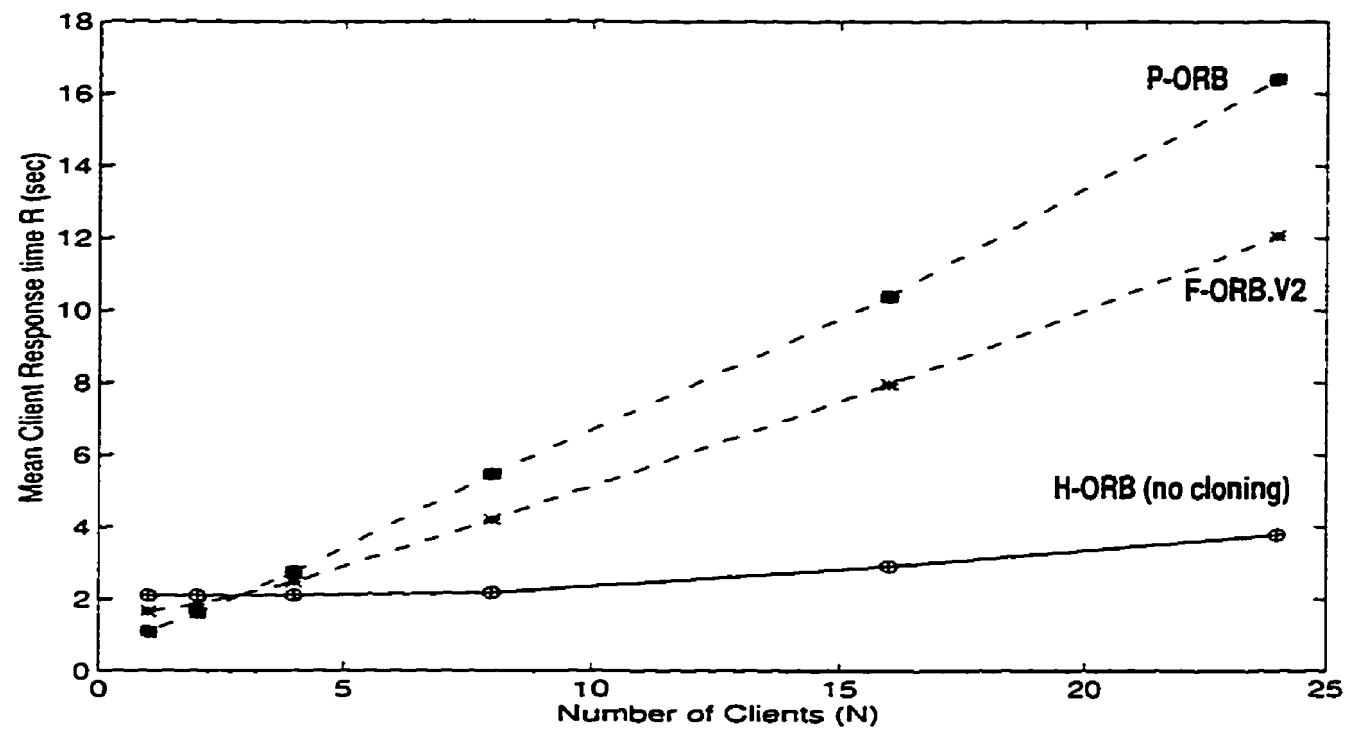

Figure C-1: The Response Time of the H-ORB, the F-ORB.V2 and the PORB with $D=250 \mathrm{~ms}$, and no Cloning.

Degree of Cloning $=I, D=250 \mathrm{~ms}, L=150$ Bytes, $S_{A}=10 \mathrm{~ms}, S_{B}=15 \mathrm{~ms}$

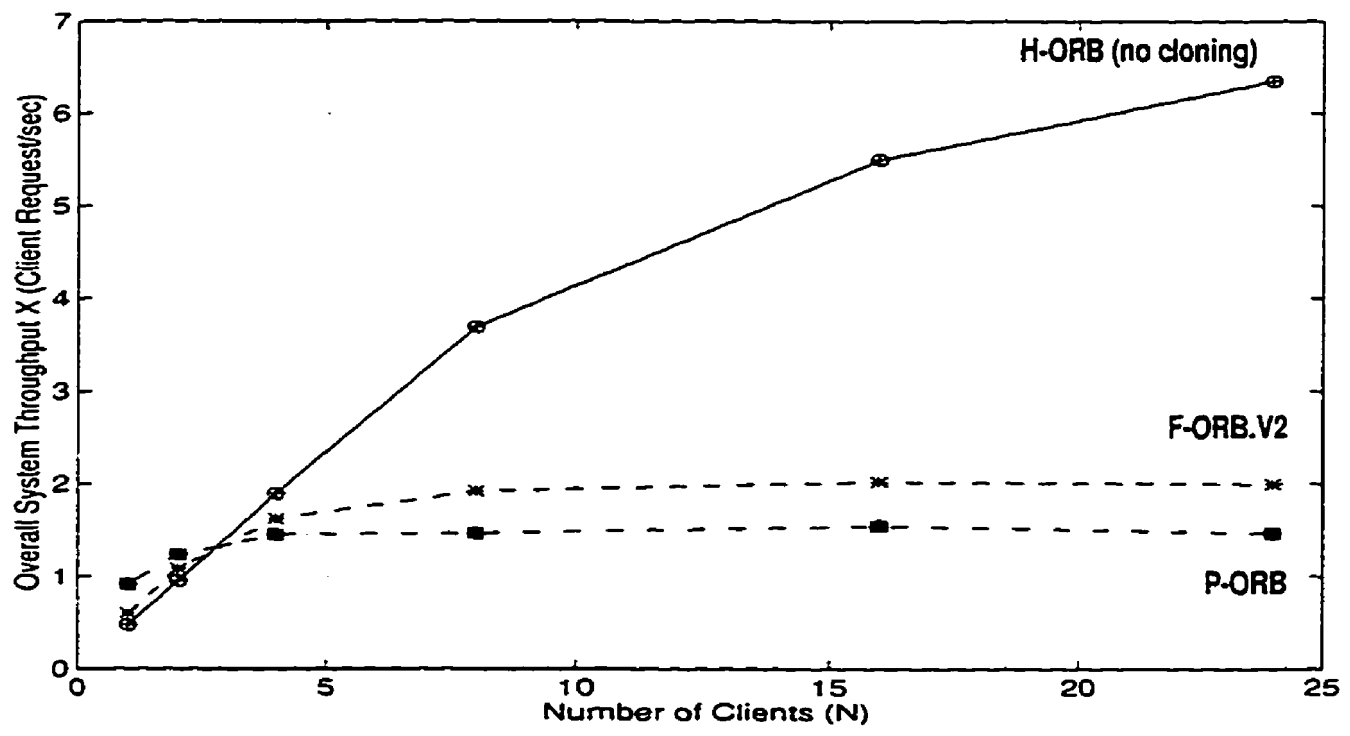

Figure C-2: The Throughput of the H-ORB, the F-ORB.V2 and the PORB with $D=250 \mathrm{~ms}$, and no Cloning.

Degree of Cloning $=I, D=250 \mathrm{~ms}, \mathrm{~L}=150$ Bytes, $S_{A}=10 \mathrm{~ms}, S_{B}=15 \mathrm{~ms}$ 


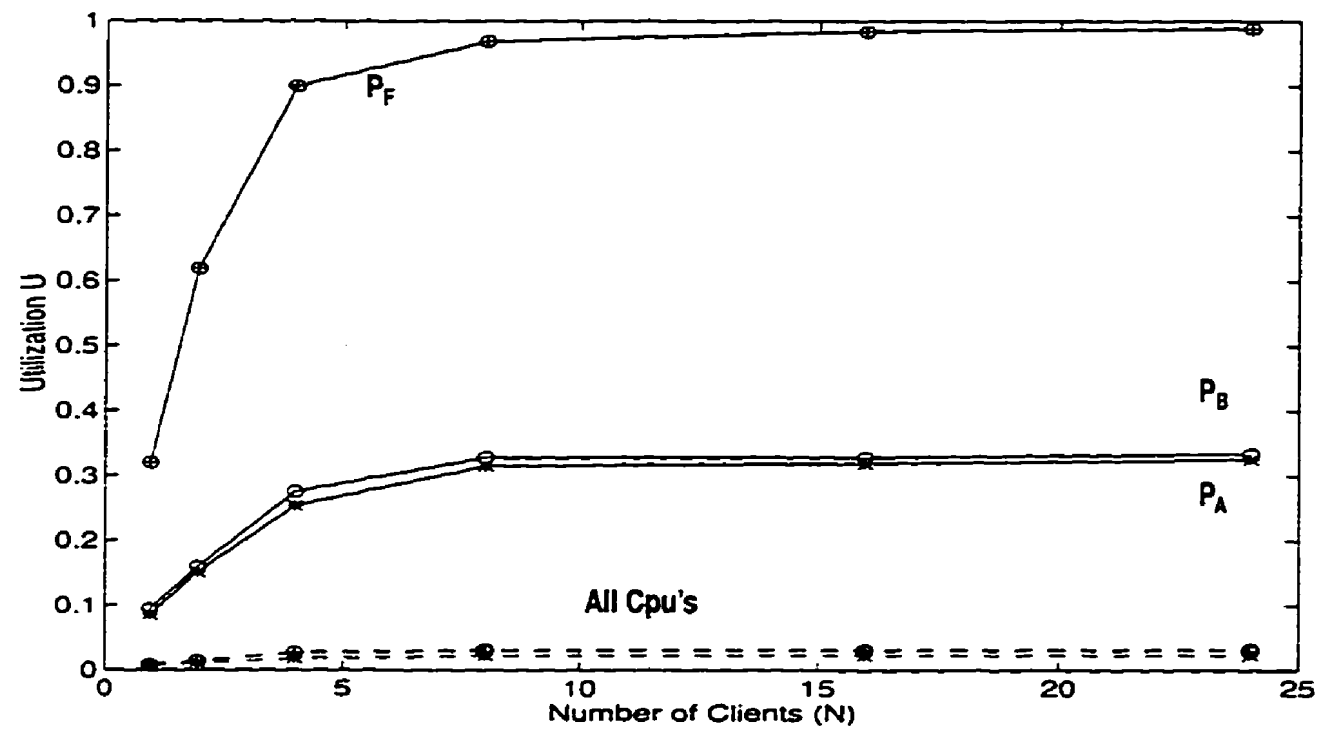

Figure C-3: Cpu \&Process Utilizations of the F-ORB.V2 with D $=250 \mathrm{~ms}$, and no Cloning.

$P_{A}=$ Process Util. of Server $A, C P U_{A}=C$ pu Util of Server $A, P_{F}=$ Process Util. of $f$-agent. Degree of Cloning $=1, D=250 \mathrm{~ms}, L=150$ Bytes, $S_{A}=10 \mathrm{~ms}, S_{B}=15 \mathrm{~ms}$ 


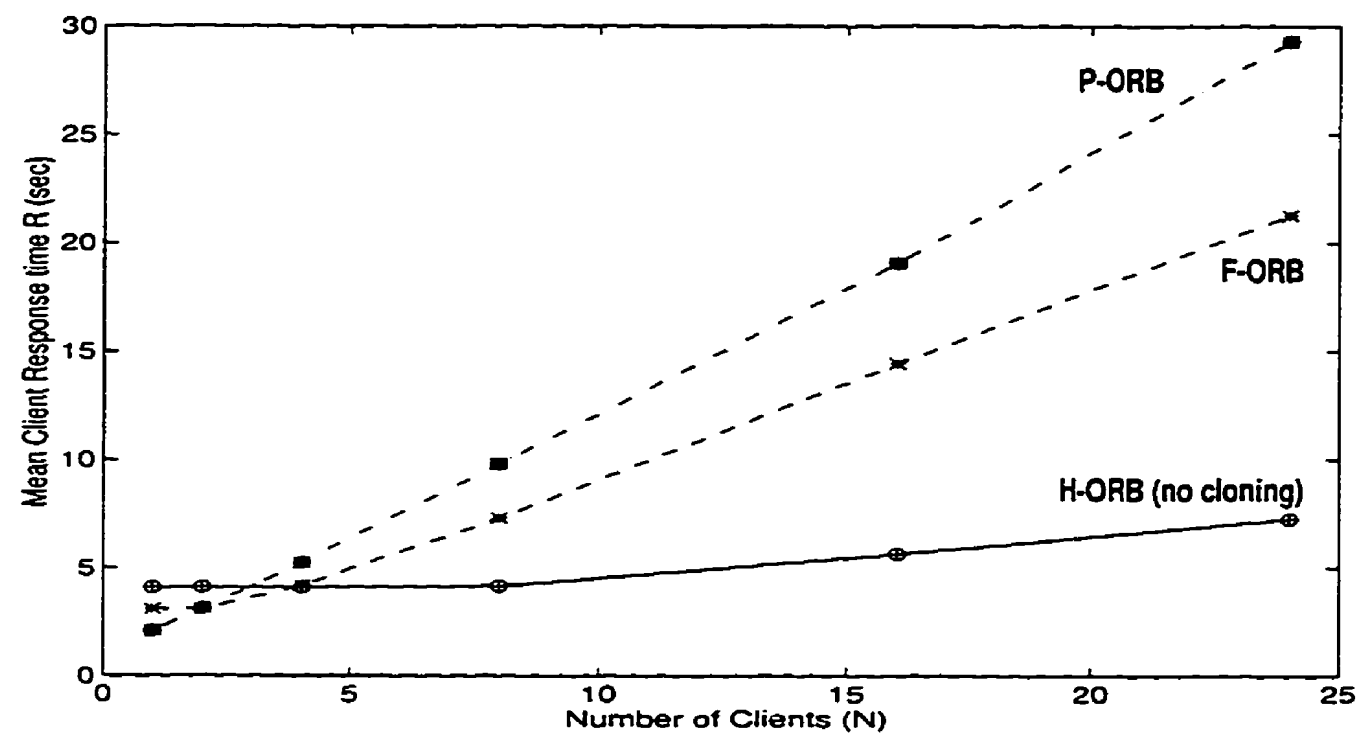

Figure C-4: The Response Time of the H-ORB, the F-ORB and the PORB with $D=500 \mathrm{~ms}$, and no Cloning.

Degree of Cloning $=1, D=500 \mathrm{~ms}, L=150$ Bytes, $S_{A}=10 \mathrm{~ms}, S_{B}=15 \mathrm{~ms}$

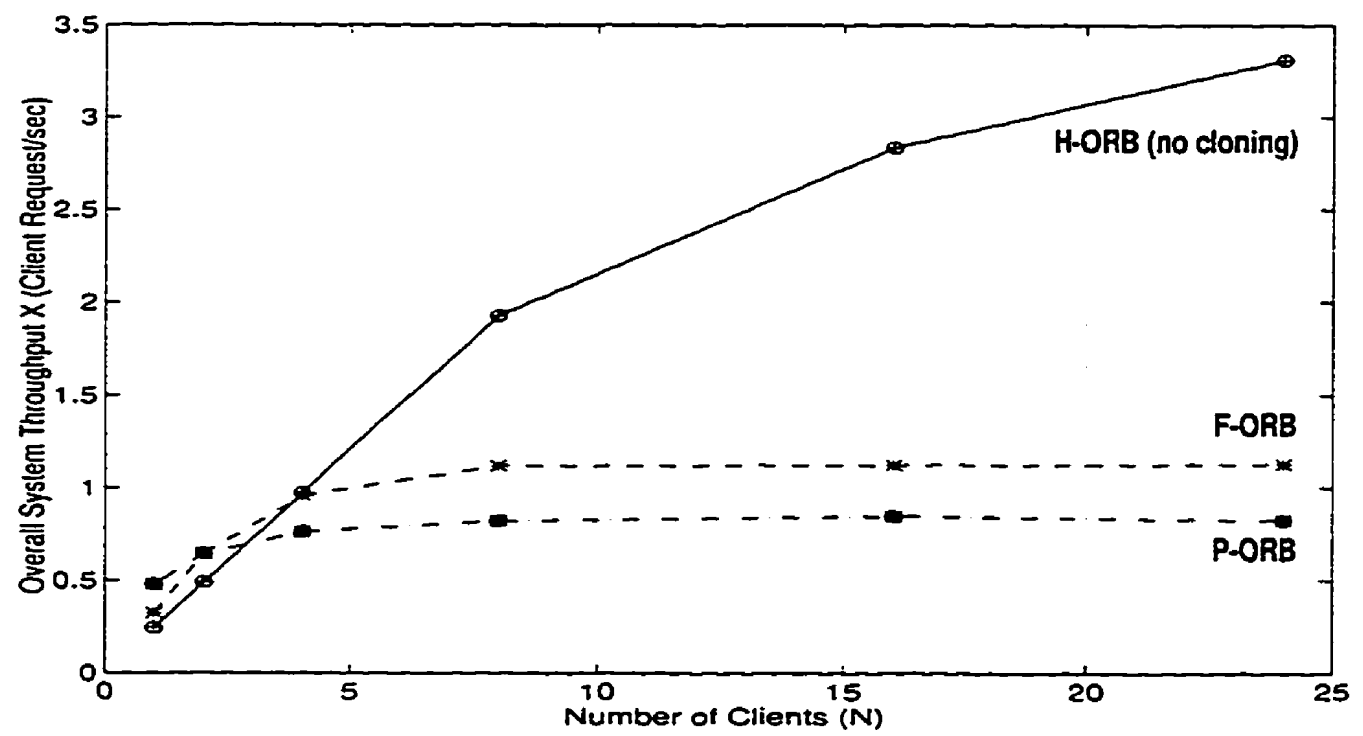

Figure C-5: The Throughput of the H-ORB, the F-ORB and the P-ORB with $D=500 \mathrm{~ms}$, and no Cloning.

Degree of Cloning= $1, D=500 \mathrm{~ms}, \mathrm{~L}=150$ Bytes, $\mathrm{S}_{A}=10 \mathrm{~ms}, \mathrm{~S}_{\mathrm{B}}=15 \mathrm{~ms}$ 


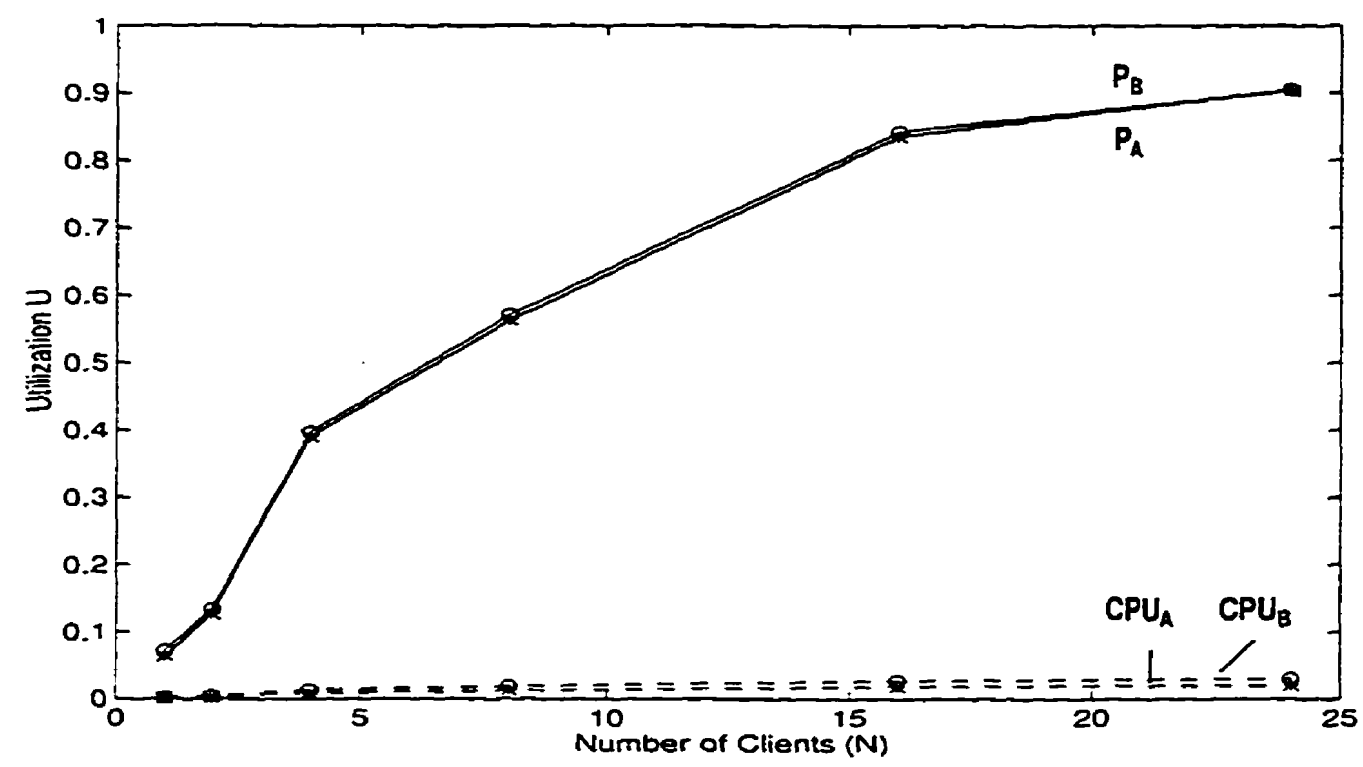

Figure C-6: Cpu and Process Utilizations of the H-ORB with $D=500 \mathrm{~ms}$, and no Cloning.

$P_{A}=$ Process Util. of Server A, CPU $U_{A}=C$ pu Util of Server $A$.

Degree of Cloning $=1, D=500 \mathrm{~ms}, L=150$ Bytes, $S_{A}=10 \mathrm{~ms}, S_{B}=15 \mathrm{~ms}$

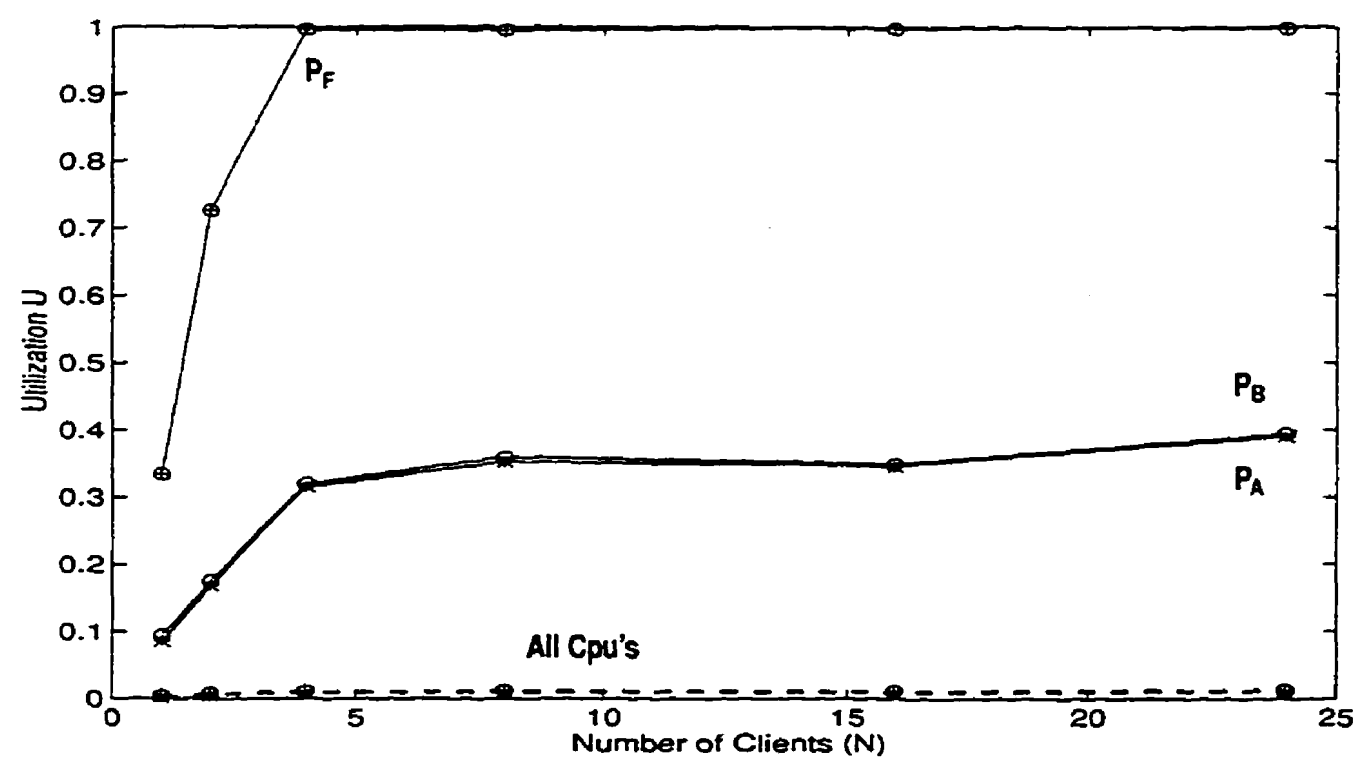

Figure C-7: Cpu \&Process Utilizations of the F-ORB with D $=500$ ms, and no Cloning.

$P_{A}=$ Process Util. of Server $A, C P U_{A}=C p u$ Uril of Server $A, P_{F}=$ Process Util. of f-agent.

Degree of Cloning $=1, D=500 \mathrm{~ms}, L=150$ Bytes, $S_{A}=10 \mathrm{~ms}, S_{B}=15 \mathrm{~ms}$ 


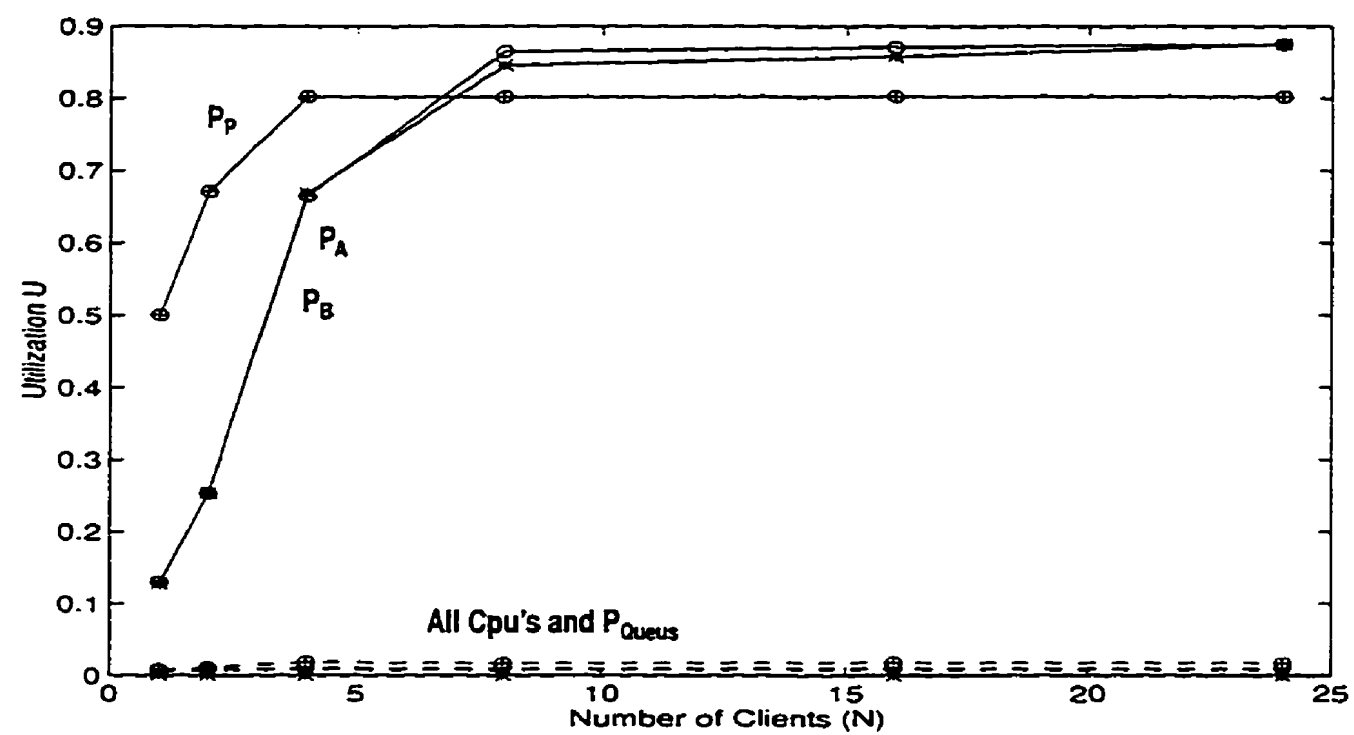

Figure C-8: Cpu \& Process Utilizations of the P-ORB with $D=500 \mathrm{~ms}$, and no Cloning Degrees.

$P_{A}=$ Process Util. of Server $A, C P U_{A}=C$ pu Util of Server $A, P_{P}=$ Process Util. of p-agent. $P$ Qveues $=$ Process Util. of dispatcher, Degree of Cloning $=1, D=500 \mathrm{~ms}, L=150$ Bytes, $\mathrm{S}_{\mathrm{A}}=10 \mathrm{~ms}, \mathrm{~S}_{\mathrm{B}}=15 \mathrm{~ms}$ 


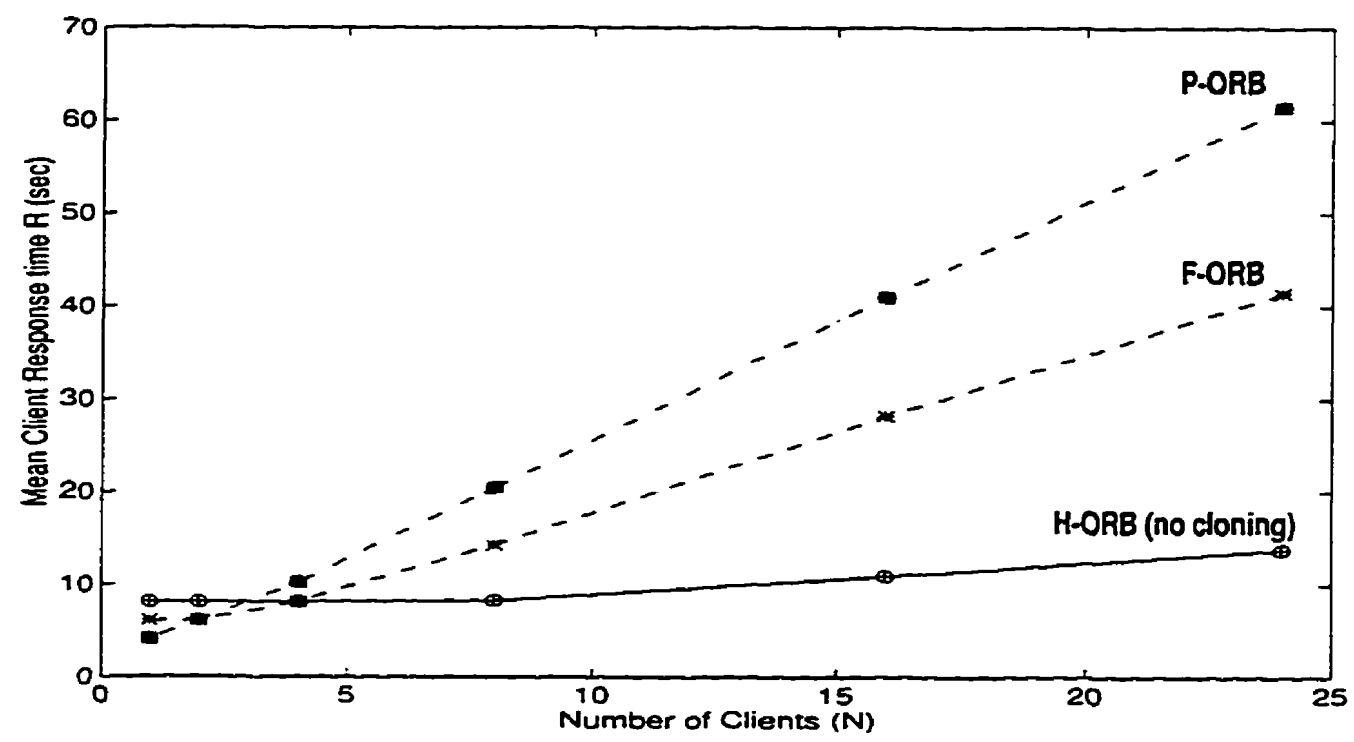

Figure C-9: The Response Time of the H-ORB, the F-ORB and the P$O R B$ with $D=1000 \mathrm{~ms}$, and no Cloning.

Degree of Cloning $=1, D=1000 \mathrm{~ms}, L=150$ Bytes, $S_{A}=10 \mathrm{~ms}, S_{B}=15 \mathrm{~ms}$

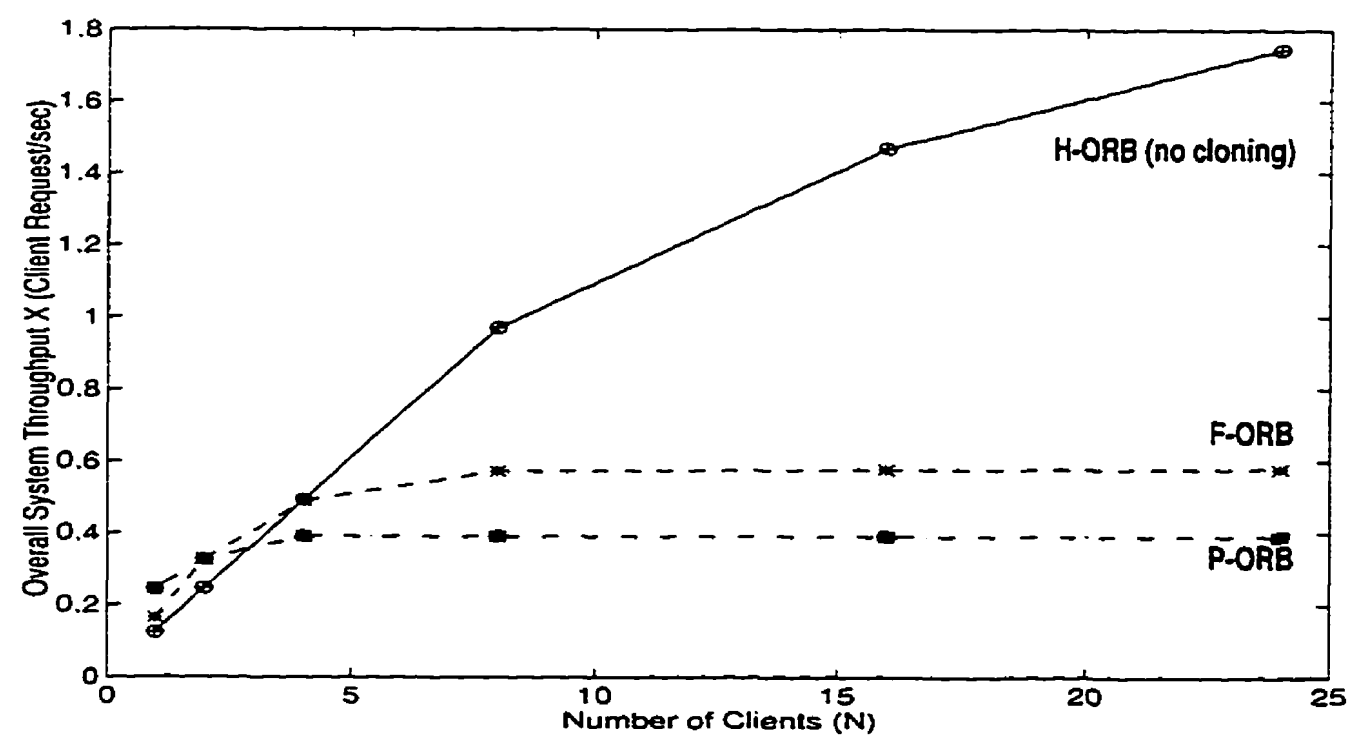

Figure C-10: The Throughput of the H-ORB, the F-ORB and the P-ORB with $D=1000 \mathrm{~ms}$, and no Cloning.

Degree of Cloning $=1, D=1000 \mathrm{~ms}, L=150$ Bytes, $S_{A}=10 \mathrm{~ms}, S_{B}=15 \mathrm{~ms}$ 


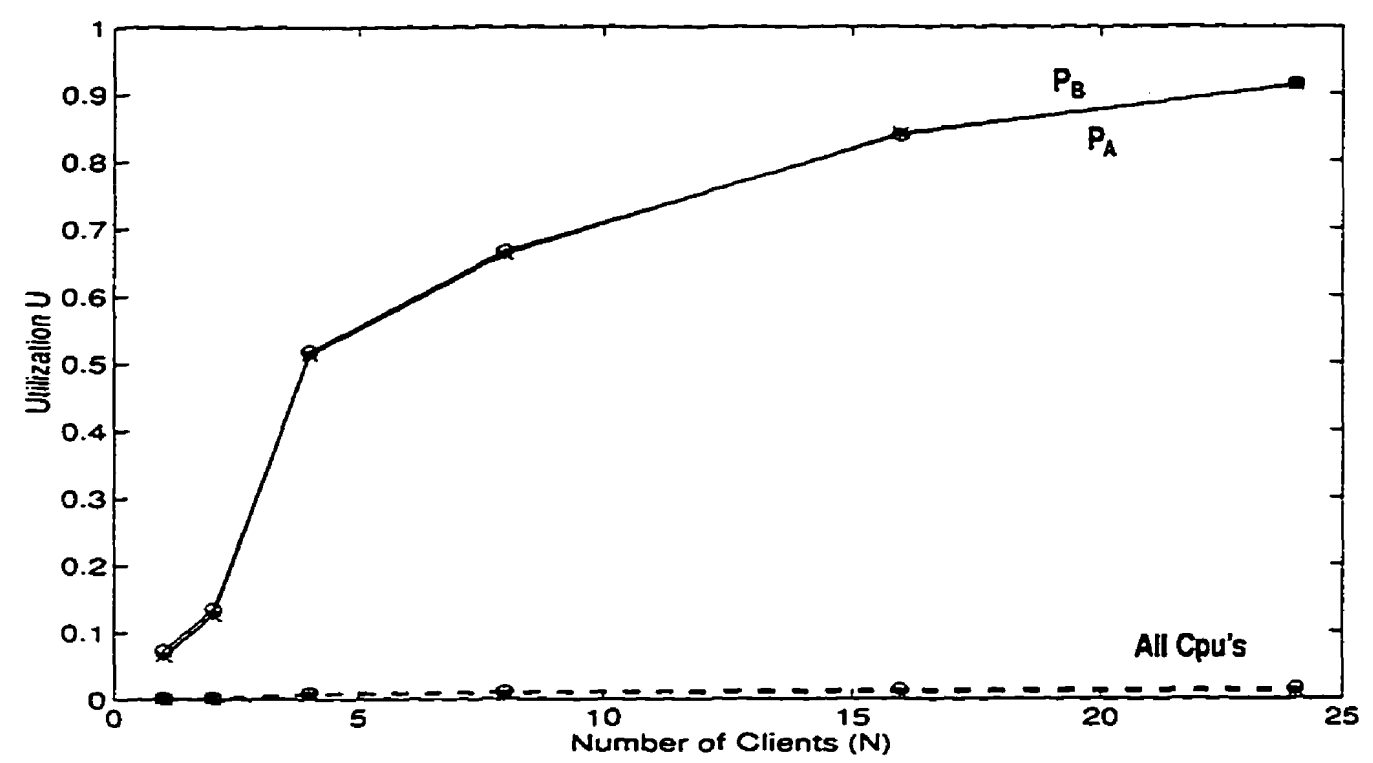

Figure C-11: Cpu and Process Utilizations of the H-ORB with $D=1000$ ms, and no Cloning.

$P_{A}=$ Process Util. of Server $A, C P U_{A}=C p u$ Util of Server $A$.

Degree of Cloning $=1, D=1000 \mathrm{~ms}, L=150$ Bytes, $S_{A}=10 \mathrm{~ms}, S_{B}=15 \mathrm{~ms}$

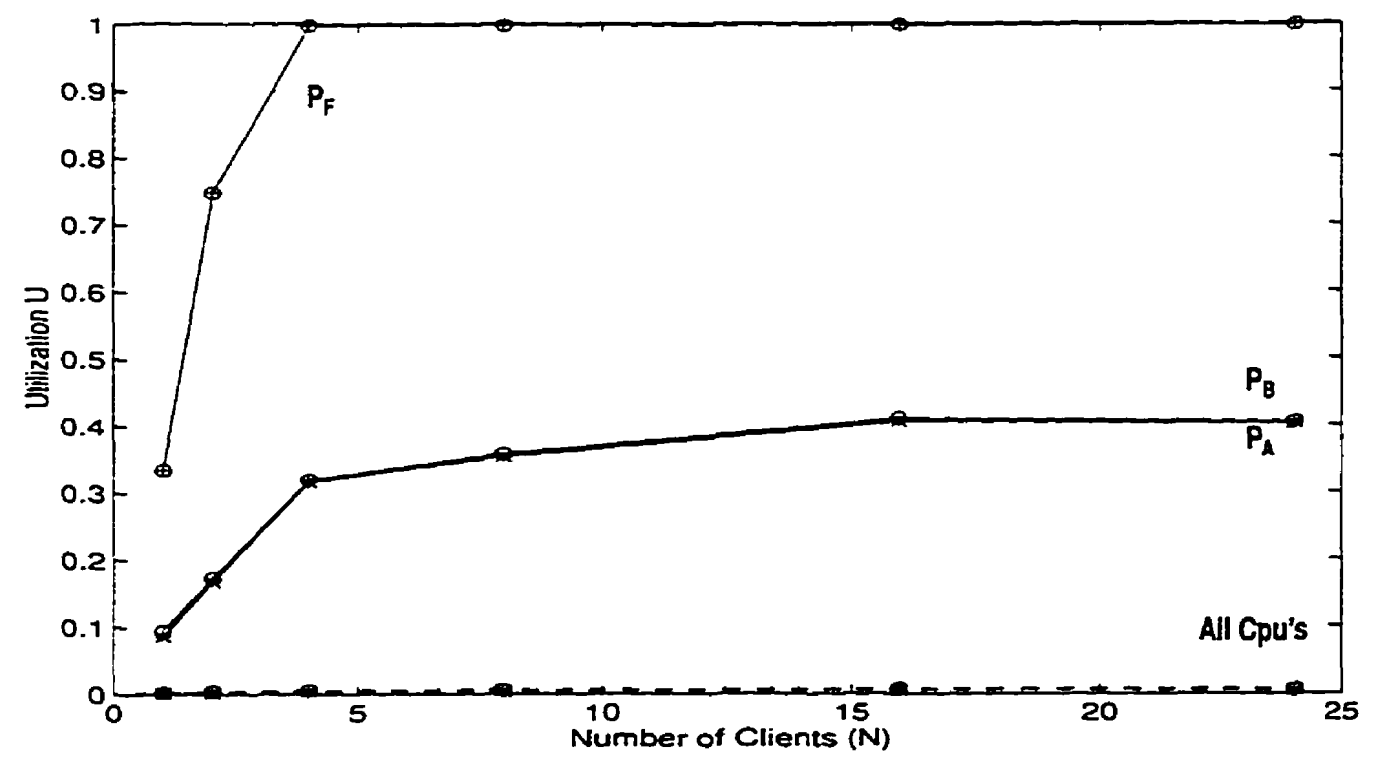

Figure C-12: Cpu \&Process Utilizations of the F-ORB with $D=1000 \mathrm{~ms}$, and no Cloning.

$P_{A}=$ Process Util. of Server $A, C P U_{A}=C$ pu Util of Server $A, P_{F}=$ Process Util. of f-agent. Degree of Cloning $=1, D=1000 \mathrm{~ms}, L=150$ Bytes, $S_{A}=10 \mathrm{~ms}, S_{B}=15 \mathrm{~ms}$ 


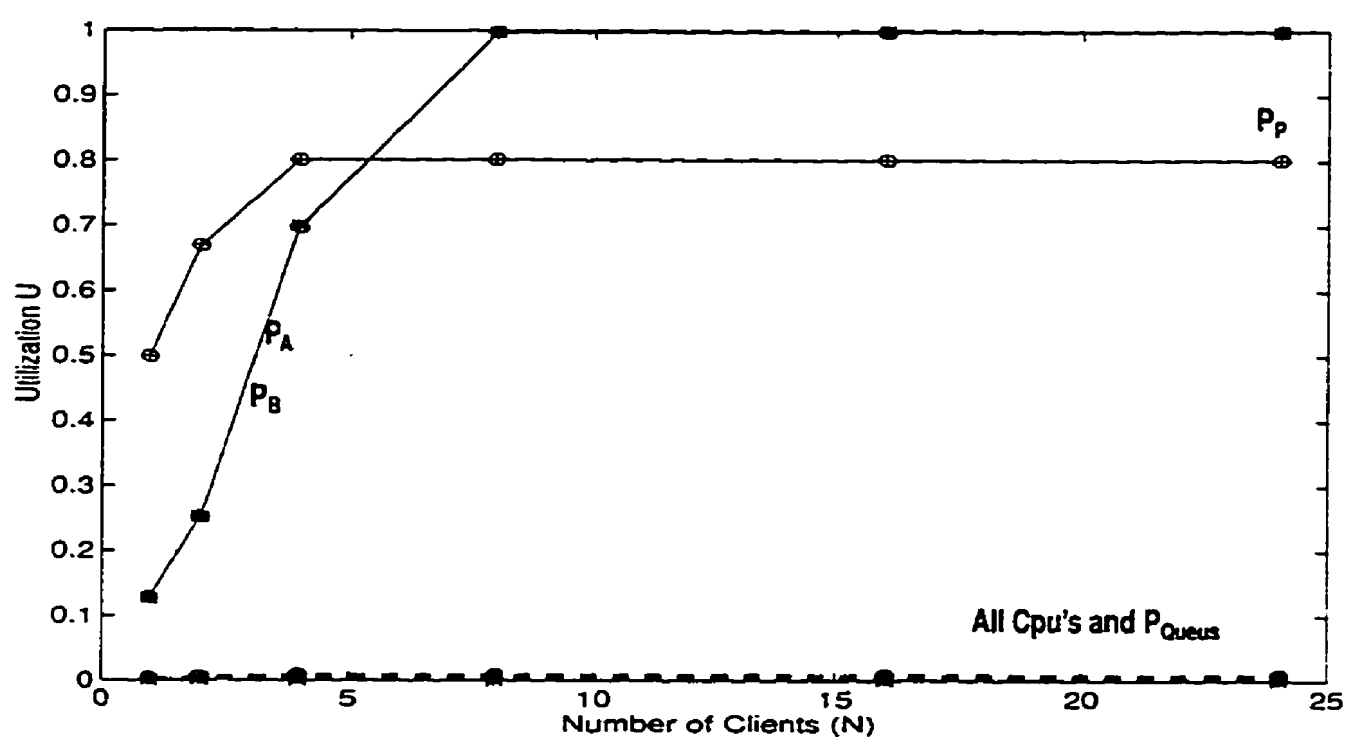

Figure C-13: Cpu \&Process Utilizations of the P-ORB with D $=1000$ ms, and no Cloning Degrees.

$P_{A}=$ Process Util. of Server $A, C P U_{A}=C p u$ Util of Server $A, P_{P}=$ Process Util. of p-agent.

$P_{\text {Qucues }}=$ Process Util. of dispatcher, Degree of Cloning $=I, D=1000 \mathrm{~ms}, L=150$ Bytes,

$\mathrm{S}_{\mathrm{A}}=10 \mathrm{~ms}, \mathrm{~S}_{\mathrm{B}}=15 \mathrm{~ms}$ 


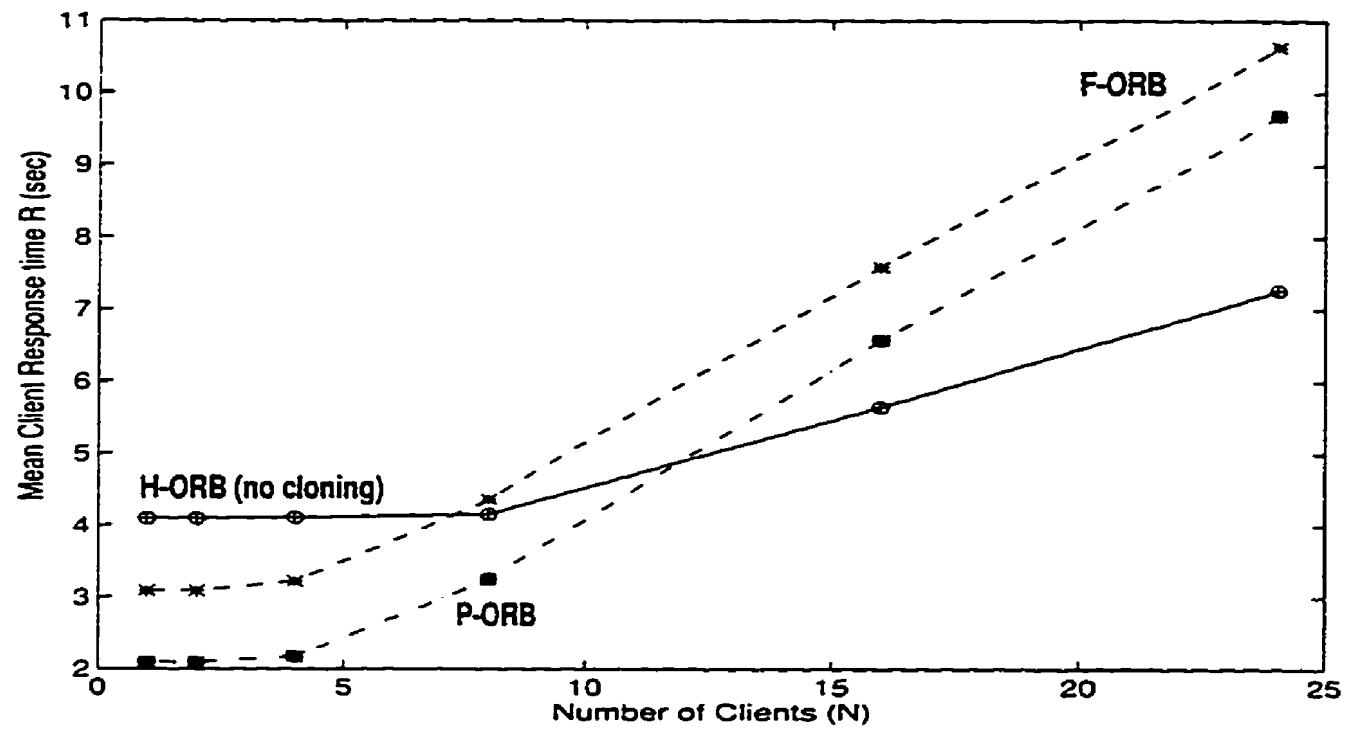

Figure C-14: The Response Time of the H-ORB, the F-ORB and the P$O R B$ with $D=500 \mathrm{~ms}$, and 4 Cloning degrees.

Degree of Cloning $=4, D=500 \mathrm{~ms}, L=150$ Bytes, $S_{A}=10 \mathrm{~ms}, S_{B}=15 \mathrm{~ms}$

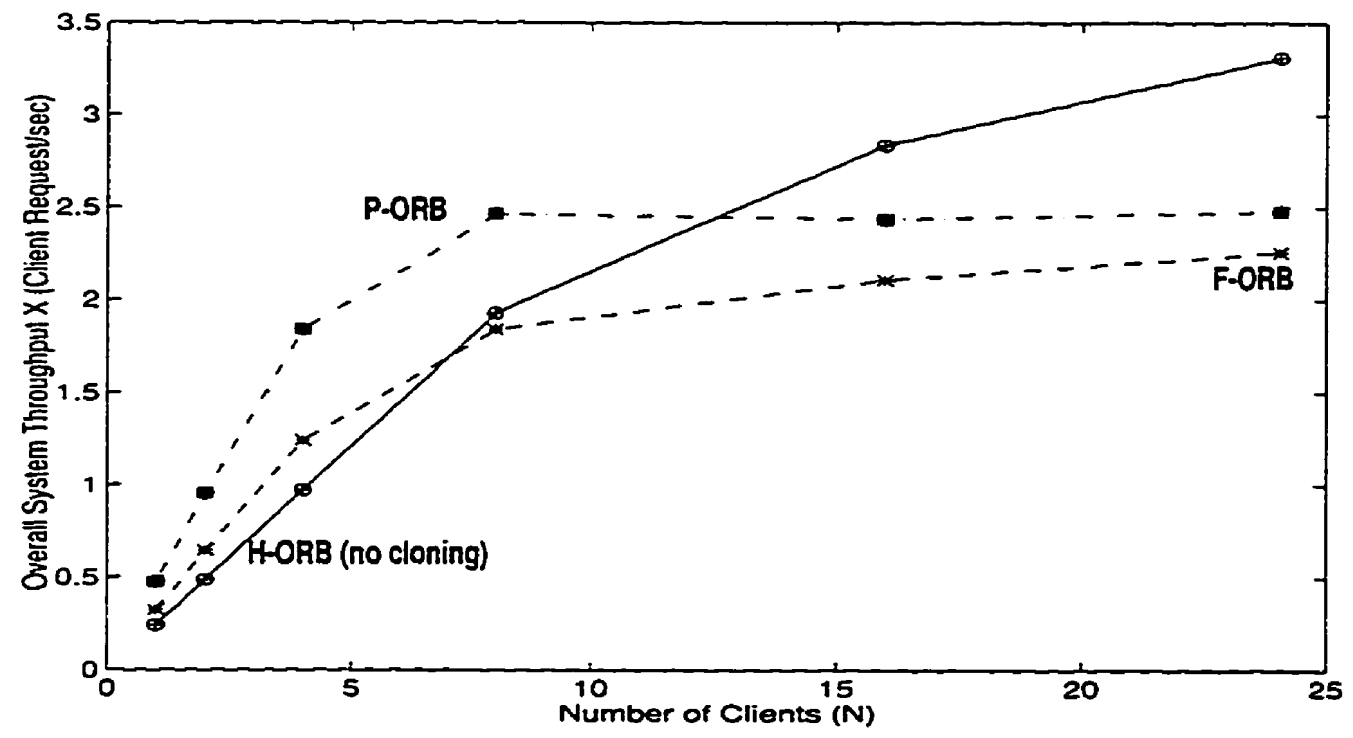

Figure C-15: The Throughput of the H-ORB, the F-ORB and the P-ORB with $D=500 \mathrm{~ms}$, and 4 Cloning degrees.

Degree of Cloning $=4, D=500 \mathrm{~ms}, L=150$ Bytes, $S_{A}=10 \mathrm{~ms}, S_{B}=15 \mathrm{~ms}$ 


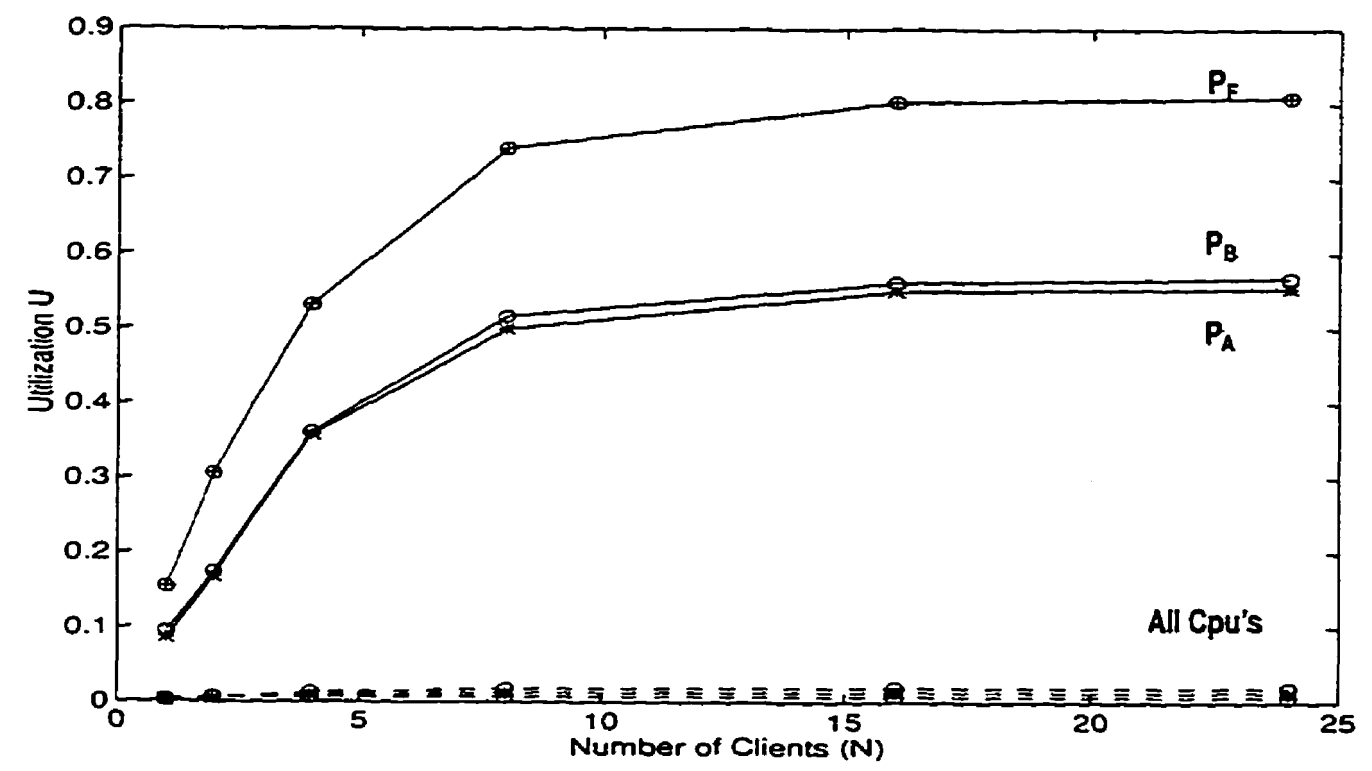

Figure C-16: Cpu \&Process Utilizations of the F-ORB with $D=500 \mathrm{~ms}$, and 4 Cloning degrees.

$P_{A}=$ Process Util. of Server A. CPU ${ }_{A}=C p u$ Util of Server A., $P_{F}=$ Process Util. of f-agent. Degree of Cloning $=4, D=500 \mathrm{~ms}, L=150$ Bytes, $S_{A}=10 \mathrm{~ms}, S_{B}=15 \mathrm{~ms}$

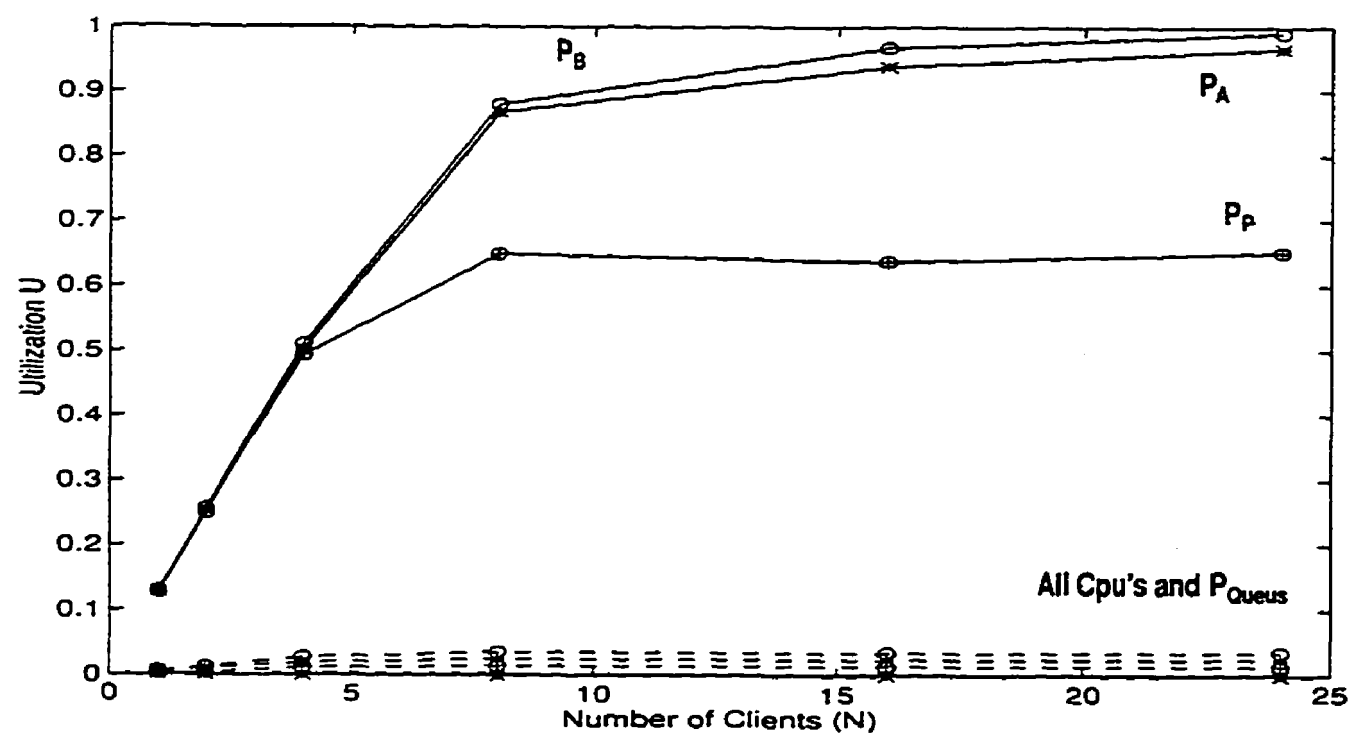

Figure C-17: Cpu \&Process Utilizations of the P-ORB with $D=500 \mathrm{~ms}$, and 4 Cloning Degrees.

$P_{A}=$ Process Util. of Server $A, C P U_{A}=C p u$ Util of Server $A, P_{p}=$ Process Util. of p-agent. $P_{\text {Queves }}=$ Process Util. of dispatcher, Degree of Cloning $=4, D=500 \mathrm{~ms}, L=150$ Bytes, $S_{A}=10 \mathrm{~ms}, S_{B}=15 \mathrm{~ms}$ 


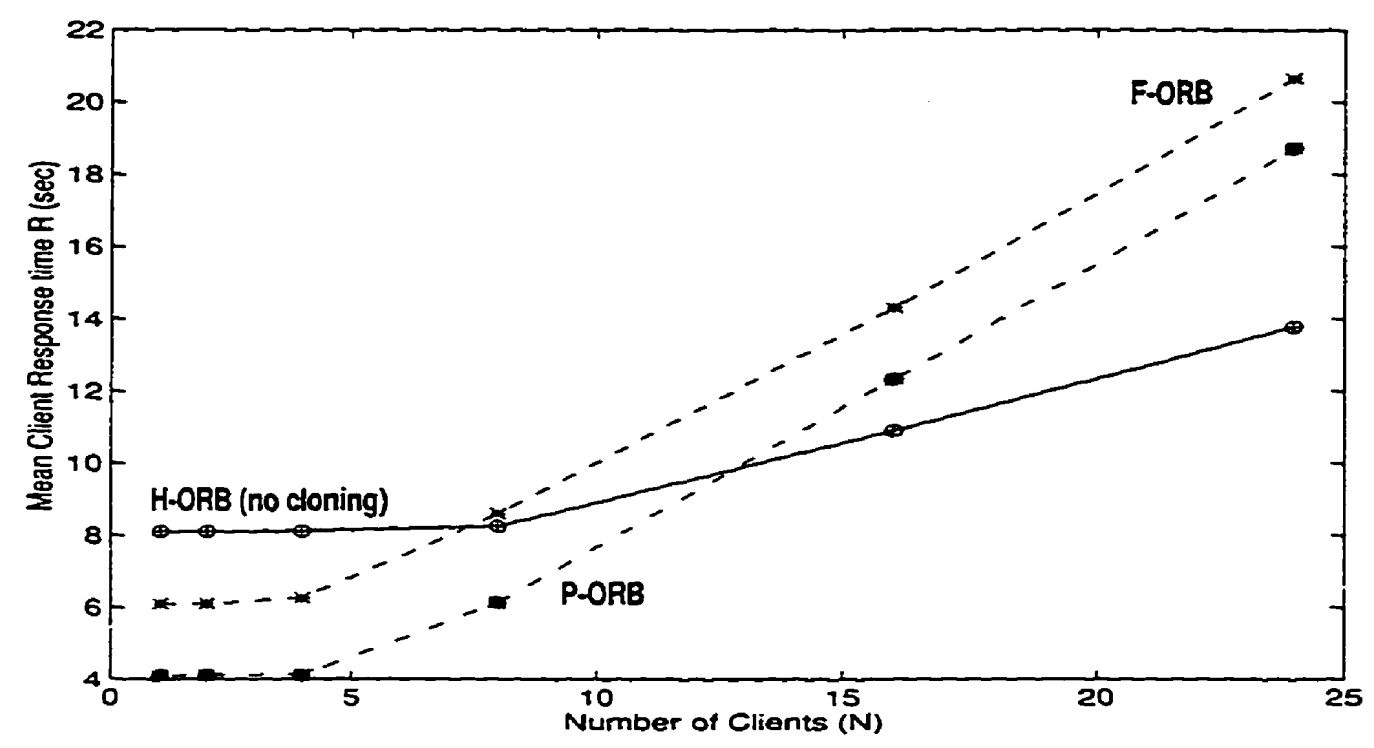

Figure C-18: The Response Time of the H-ORB, the F-ORB and the PORB with $D=1000 \mathrm{~ms}$, and 4 Cloning degrees.

Degree of Cloning $=4, D=1000 \mathrm{~ms}, L=150$ Bytes, $S_{A}=10 \mathrm{~ms}, S_{B}=15 \mathrm{~ms}$

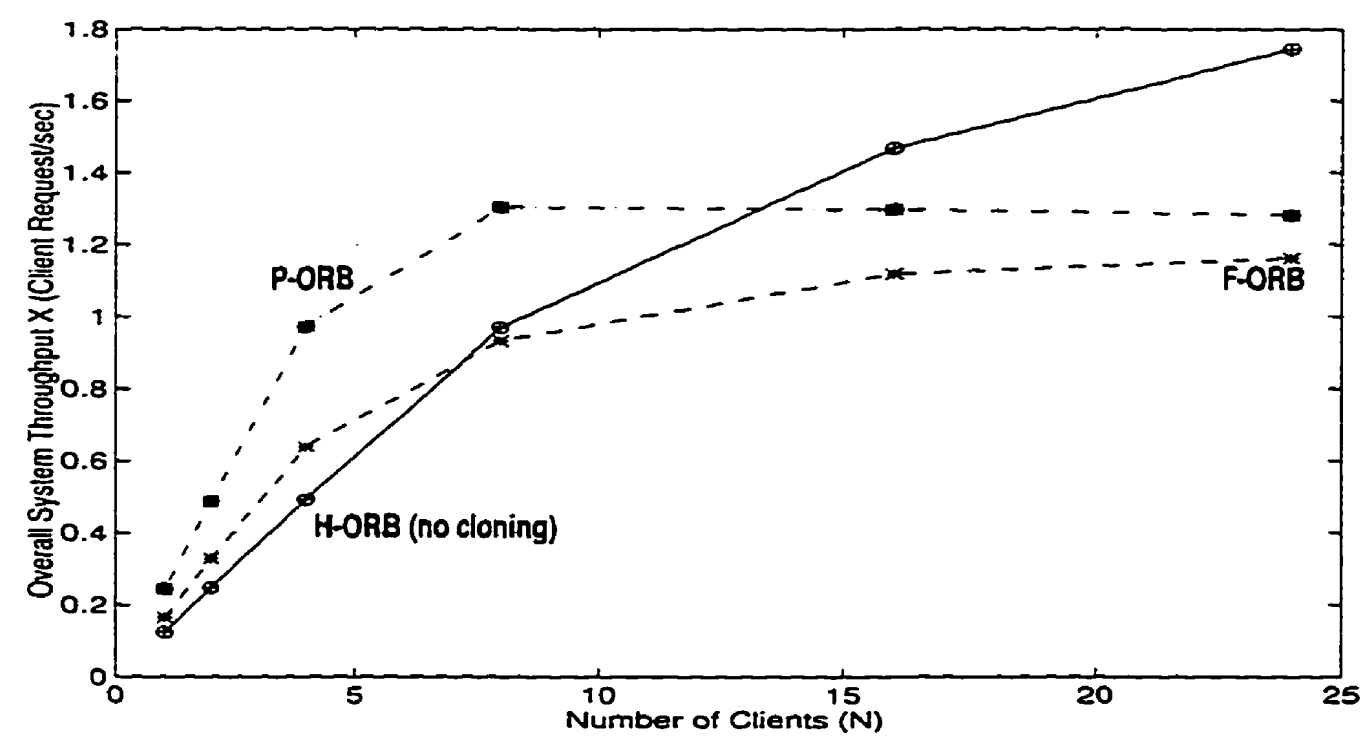

Figure C-19: The Throughput of the H-ORB, the F-ORB and the P-ORB with $D=1000 \mathrm{~ms}$, and 4 Cloning degrees.

Degree of Cloning $=4, D=1000 \mathrm{~ms}, L=150$ Bytes, $S_{A}=10 \mathrm{~ms}, S_{B}=15 \mathrm{~ms}$ 


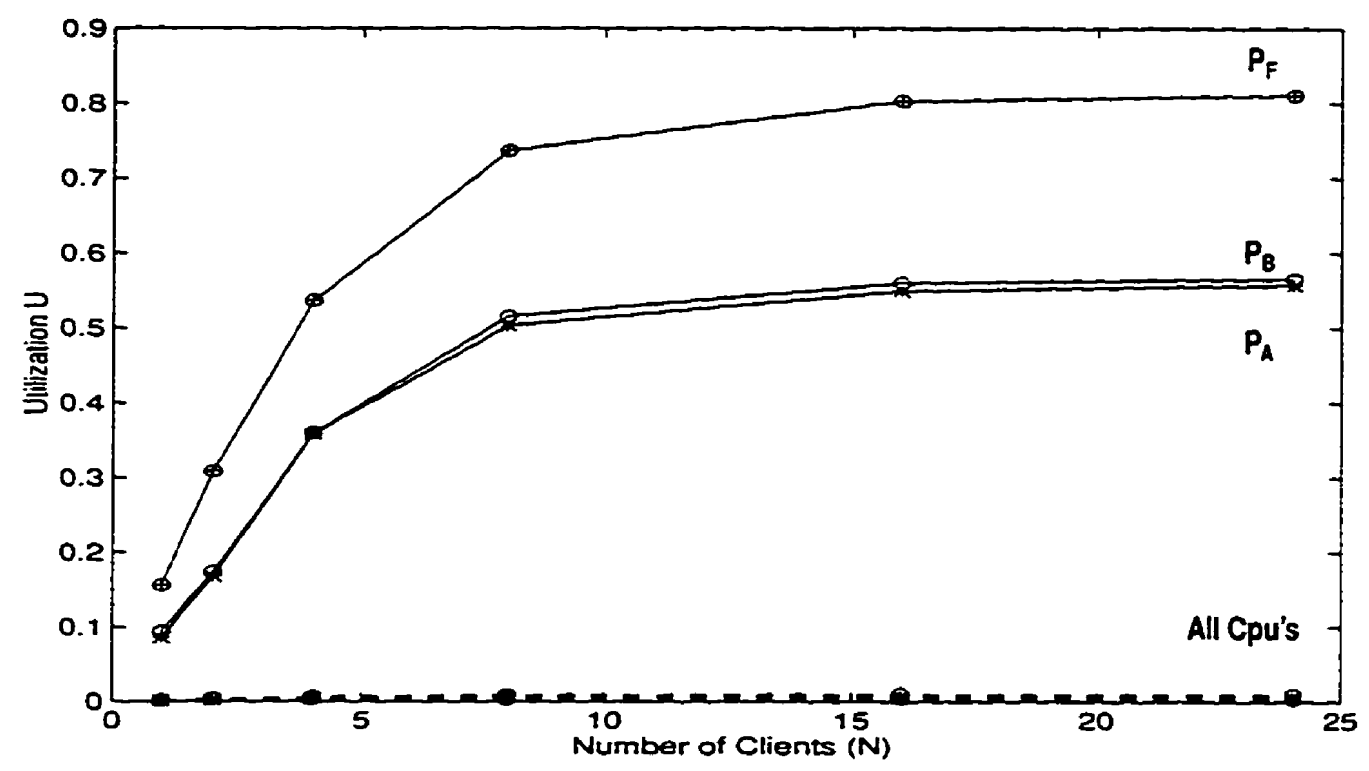

Figure C-20: Cpu \& Process Utilizations of the F-ORB with $D=1000 \mathrm{~ms}$, and 4 Cloning degrees.

$P_{A}=$ Process Util. of Server $A, C P U_{A}=C p u$ Util of Server $A, P_{F}=$ Process Util. of f-agent. Degree of Cloning $=4, D=1000 \mathrm{~ms}, L=150$ Bytes, $S_{A}=10 \mathrm{~ms}, S_{B}=15 \mathrm{~ms}$

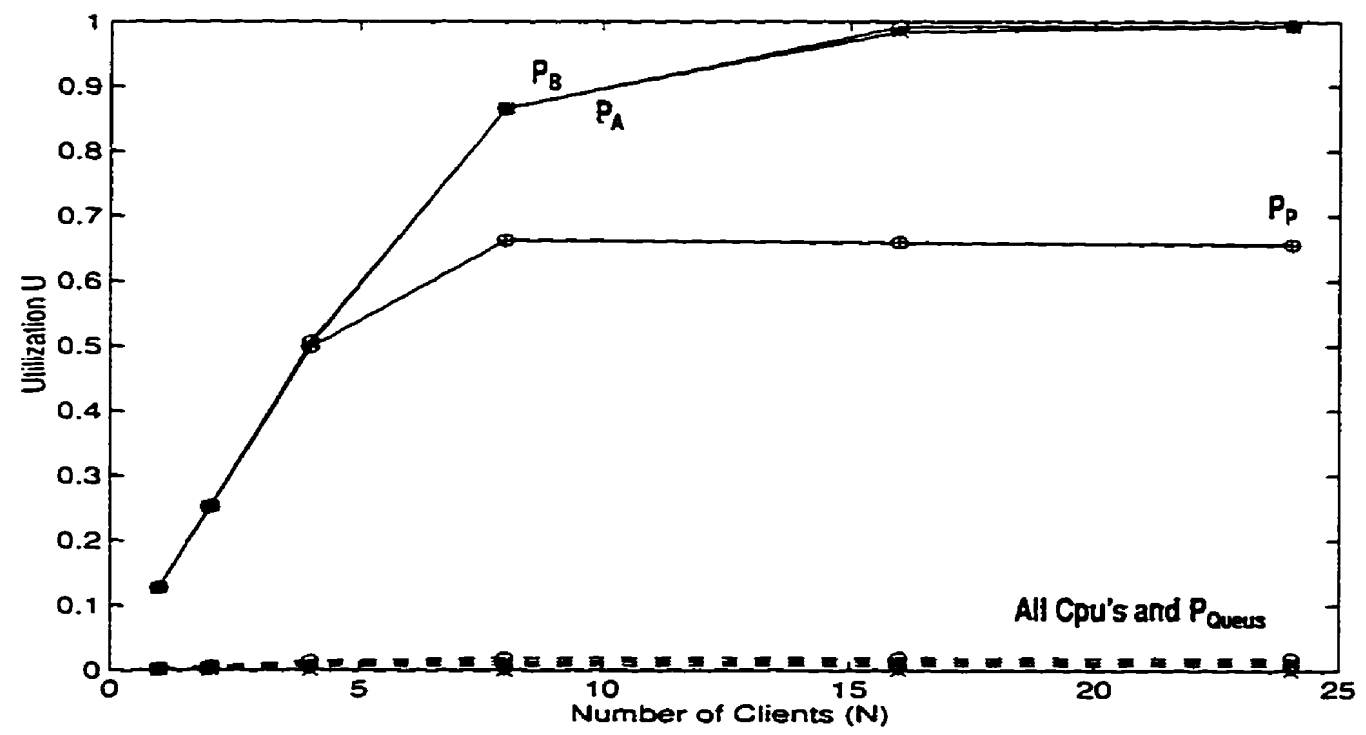

Figure C-21: Cpu \&Process Utilizations of the P-ORB with $D=1000 \mathrm{~ms}$, and 4 Cloning degrees.

$P_{A}=$ Process Util. of Server $A, C P U_{A}=C p u$ Util of Server $A, P_{p}=$ Process Util. of p-agent. $P_{\text {Queves }}=$ Process Util. of dispatcher, Degree of Cloning $=4, D=1000 \mathrm{~ms}, \mathrm{~L}=150$ Bytes, $\mathrm{S}_{\mathrm{A}}=10 \mathrm{~ms}, \mathrm{~S}_{\mathrm{B}}=15 \mathrm{~ms}$ 


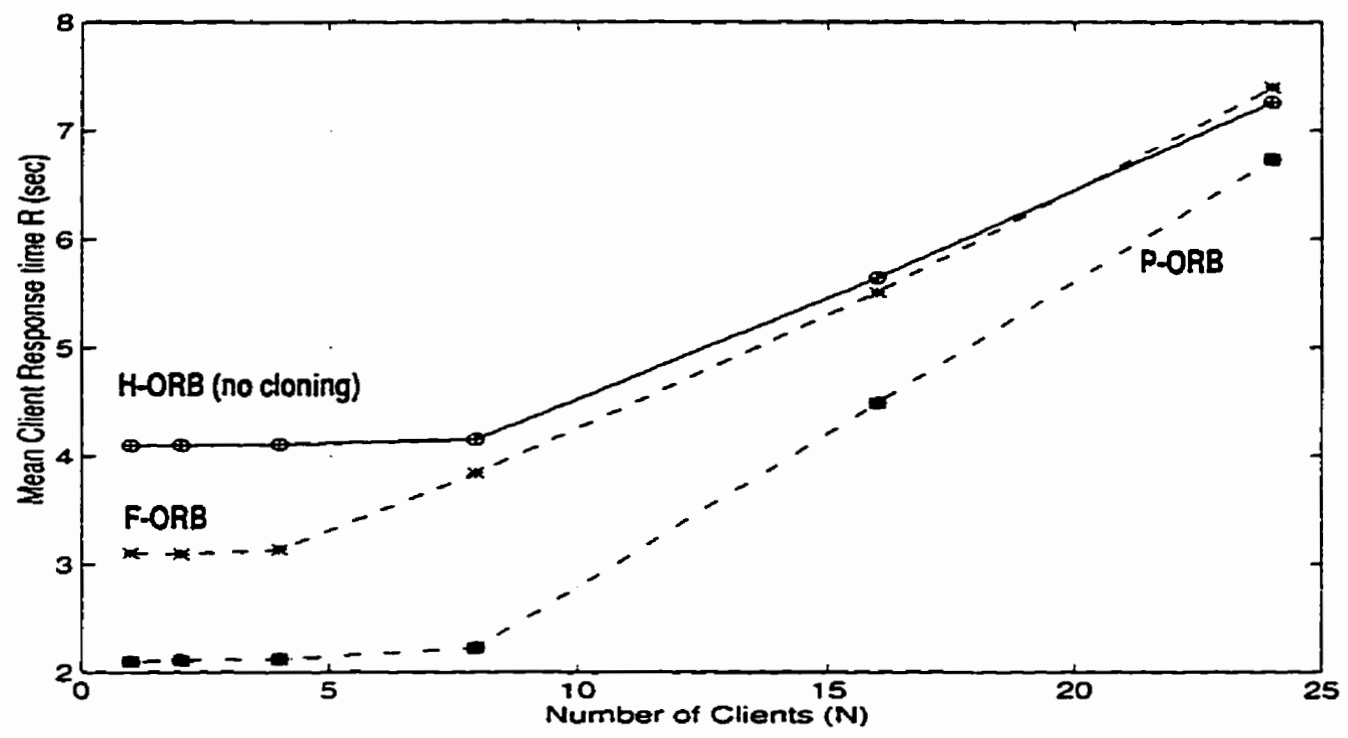

Figure C-22: The Response Time of the H-ORB, the F-ORB and the PORB with $D=500 \mathrm{~ms}$, and 8 Cloning degrees.

Degree of Cloning $=8, D=500 \mathrm{~ms}, L=150$ Bytes, $S_{A}=10 \mathrm{~ms}, S_{B}=15 \mathrm{~ms}$

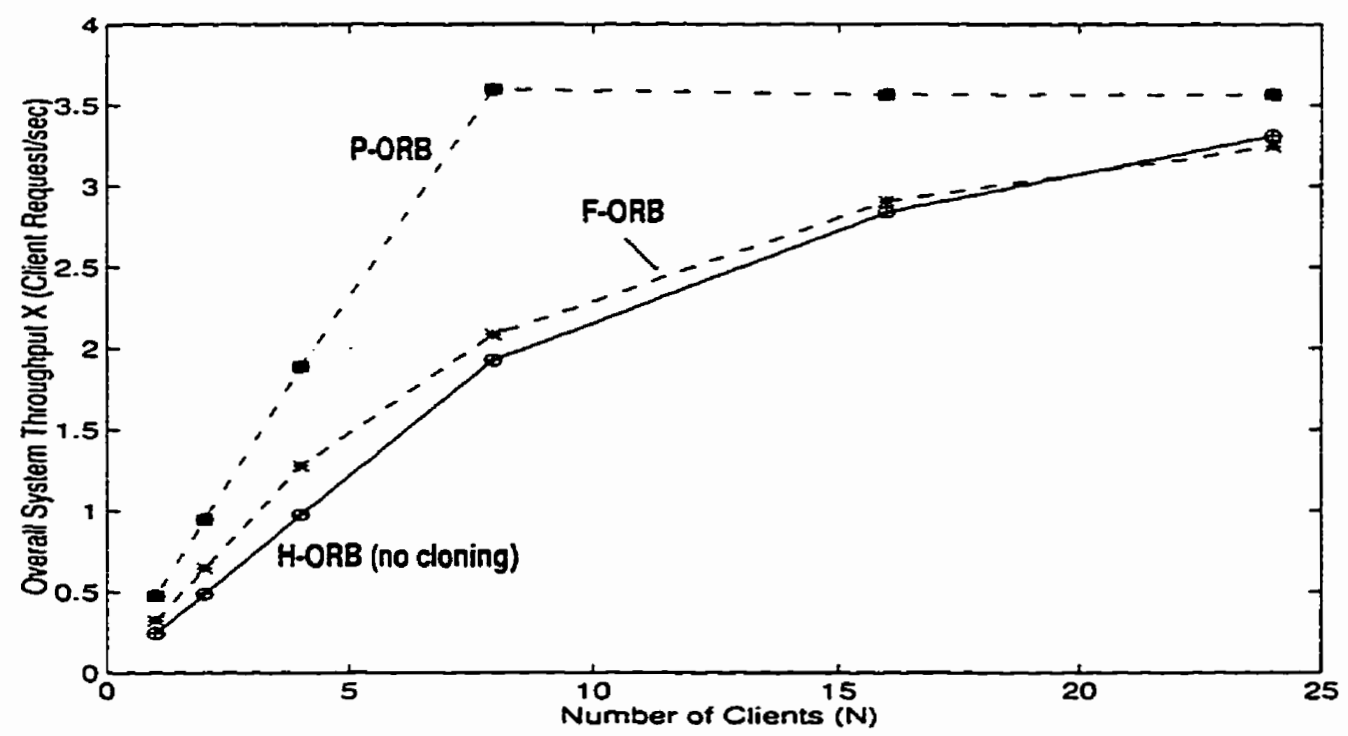

Figure C-23: The Throughput of the H-ORB, the F-ORB and the P-ORB with $\mathrm{D}=\mathbf{5 0 0} \mathrm{ms}$, and 8 Cloning degrees.

Degree of Cloning $=8 . D=500 \mathrm{~ms}, L=150$ Bytes, $S_{A}=10 \mathrm{~ms}, S_{B}=15 \mathrm{~ms}$ 


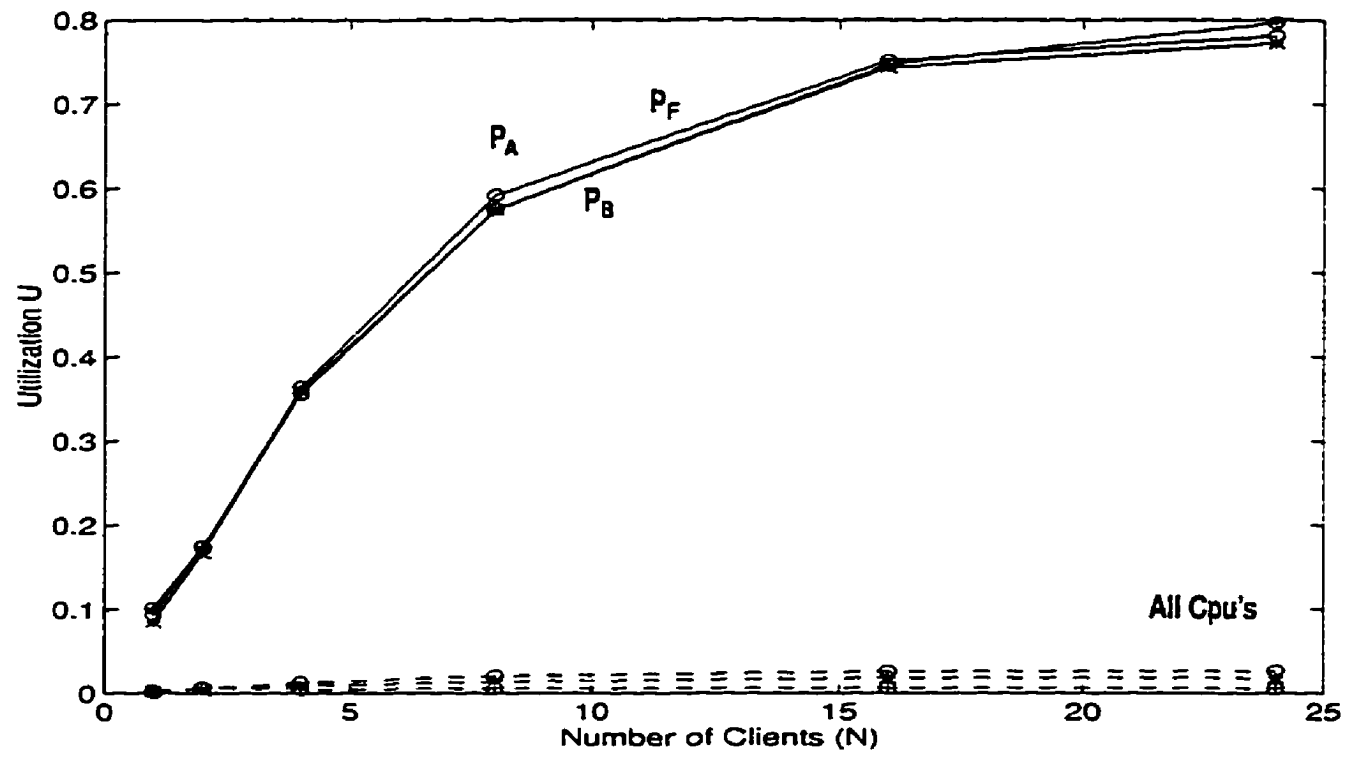

Figure C-24: Cpu \&Process Utilizations of the F-ORB with D $=500 \mathrm{~ms}$, and 8 Cloning degrees.

$P_{A}=$ Process Util. of Server $A, C P U_{A}=C p u$ Util of Server $A, P_{F}=$ Process Util. of $\mathrm{f}$-agent. Degree of Cloning $=8, D=500 \mathrm{~ms}, \mathrm{~L}=150$ Bytes, $S_{A}=10 \mathrm{~ms}, S_{B}=15 \mathrm{~ms}$

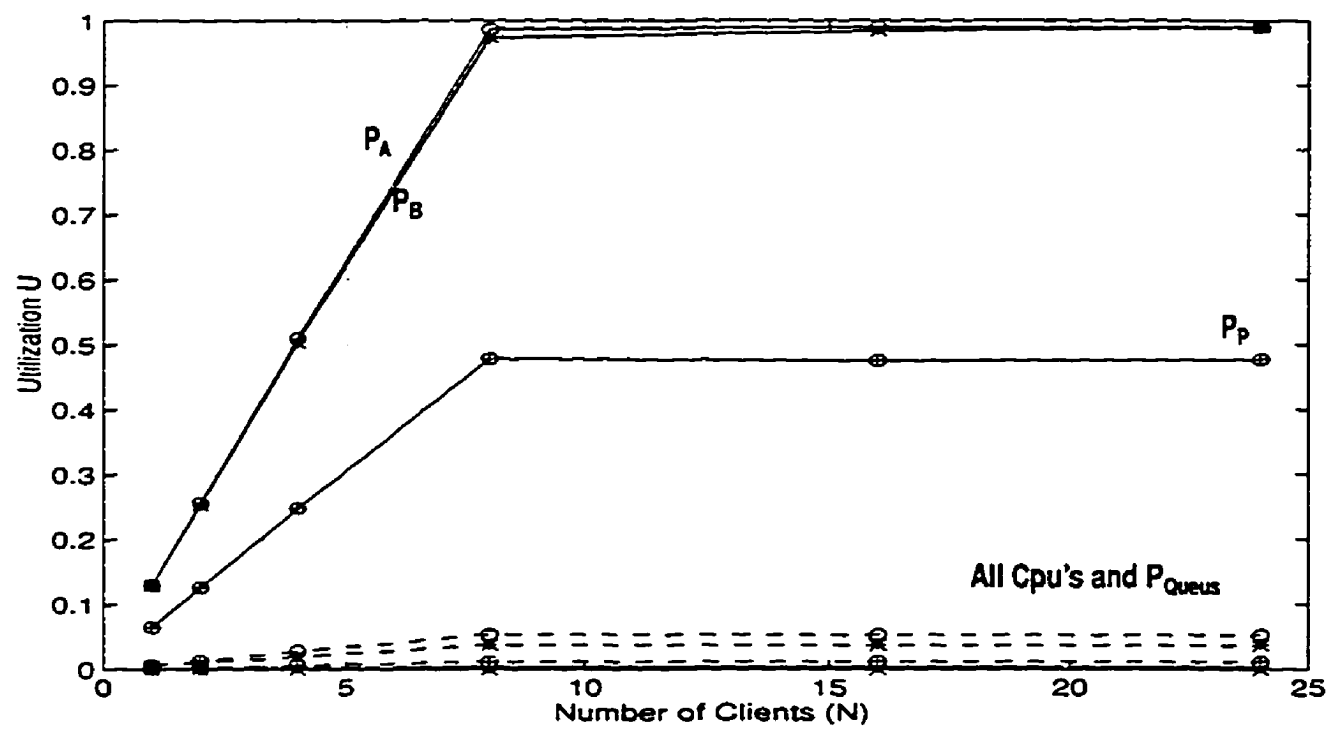

Figure C-25: Cpu \&Process Utilizations of the $P-O R B$ with $D=500$ ms, and 8 Cloning degrees.

$P_{A}=$ Process Util. of Server $A, C P U_{A}=C p u$ Util of Server $A, P_{P}=$ Process Util. of p-agent. $P_{\text {Queves }}=$ Process Util. of dispatcher, Degree of Cloning $=8, D=500 \mathrm{~ms}, \mathrm{~L}=150$ Bytes, $\mathrm{S}_{\mathrm{A}}=10 \mathrm{~ms}, \mathrm{~S}_{\mathrm{B}}=15 \mathrm{~ms}$ 


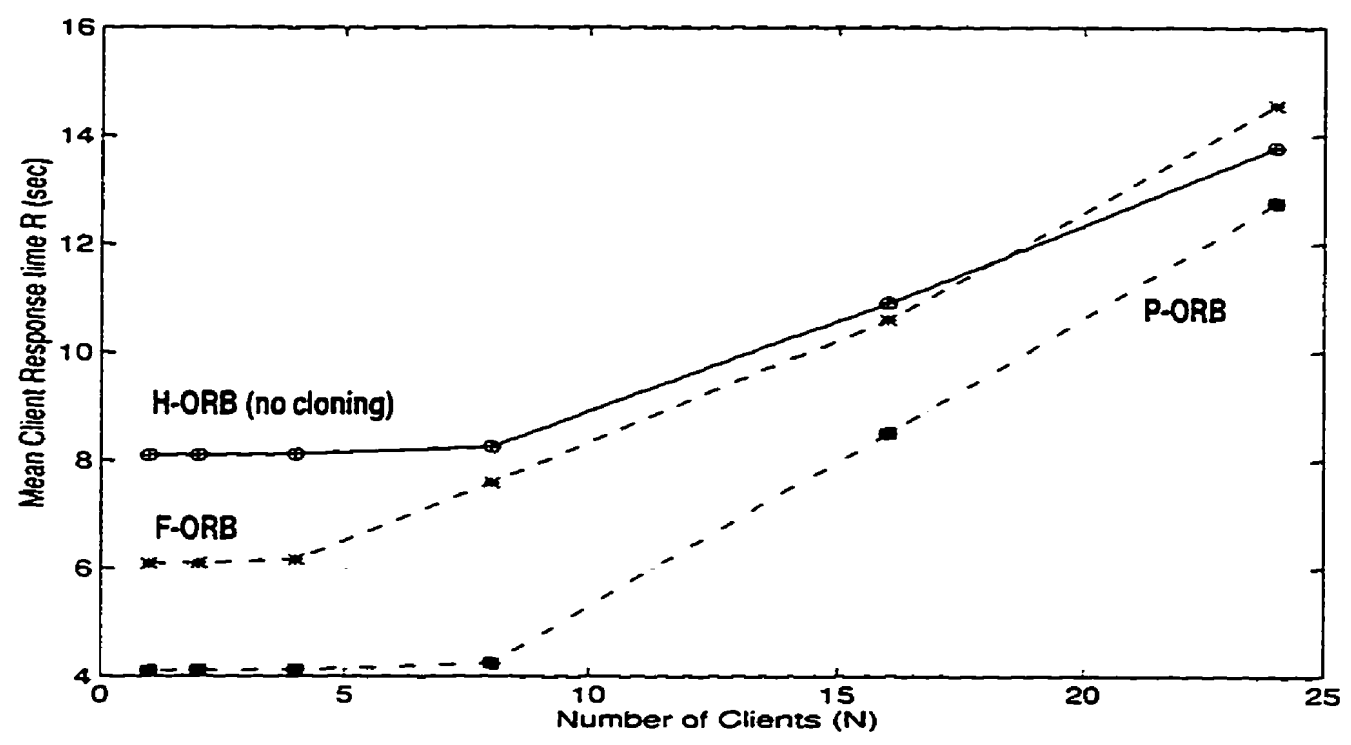

Figure C-26: The Response Time of the $\mathrm{H}-\mathrm{ORB}$, the F-ORB and the PORB with $\mathrm{D}=1000 \mathrm{~ms}$, and 8 Cloning degrees.

Degree of Cloning $=8, D=1000 \mathrm{~ms}, L=150$ Bytes, $S_{A}=10 \mathrm{~ms}, S_{B}=15 \mathrm{~ms}$

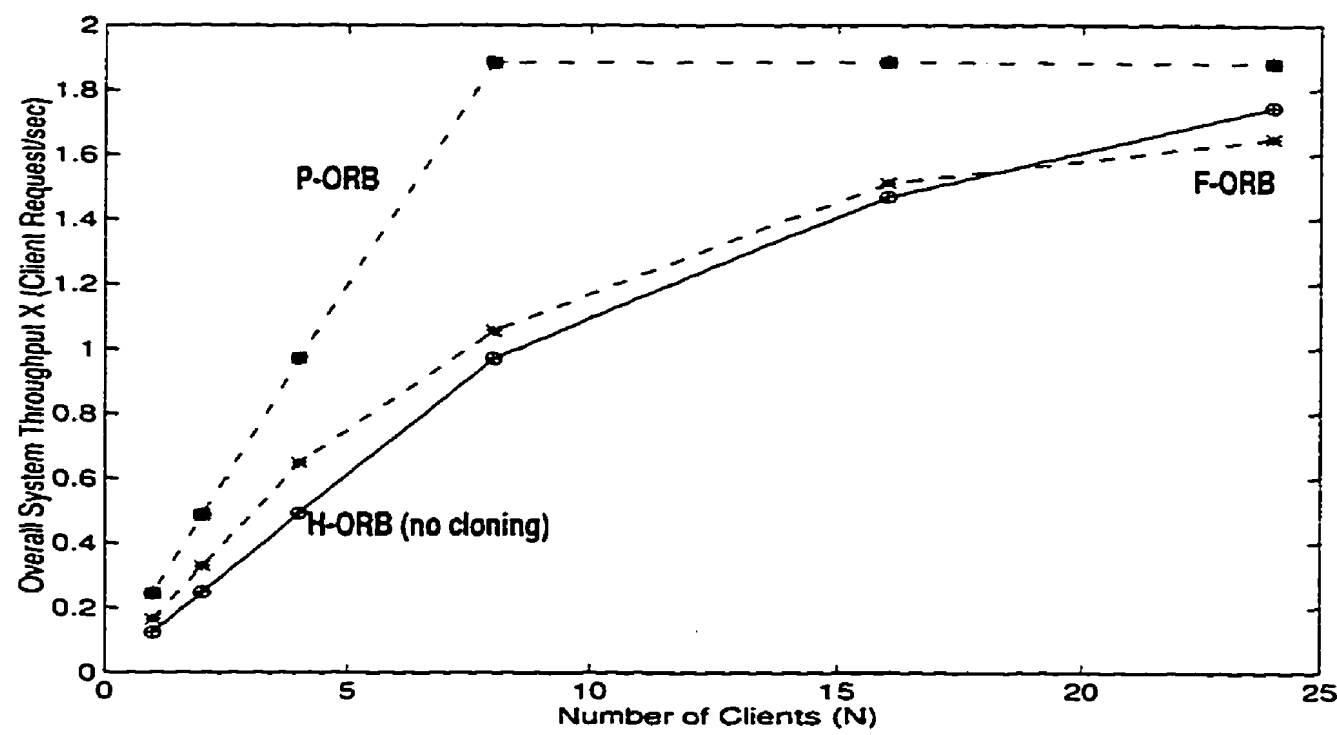

Figure C-27: The Throughput of the H-ORB, the F-ORB and the P-ORB with $D=1000 \mathrm{~ms}$, and 8 Cloning degrees.

Degree of Cloning $=8, D=1000 \mathrm{~ms}, \mathrm{~L}=150$ Bytes, $S_{A}=10 \mathrm{~ms}, S_{B}=15 \mathrm{~ms}$ 


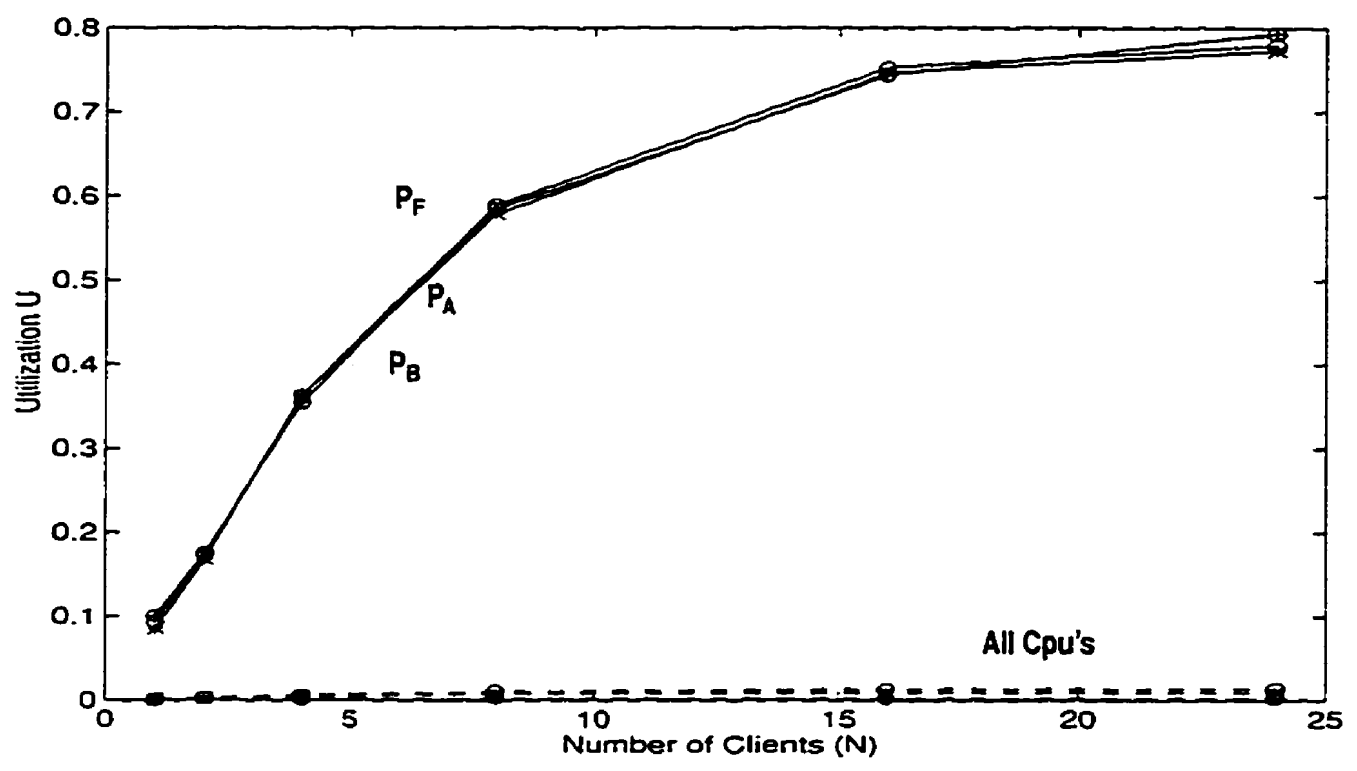

Figure C-28: Cpu \&Process Utilizations of the F-ORB with $D=1000 \mathrm{~ms}$, and 8 Cloning degrees.

$P_{A}=$ Process Util. of Server $A, C P U_{A}=C$ pu Util of Server $A, P_{F}=$ Process Util. of f-agent. Degree of Cloning $=8, D=1000 \mathrm{~ms}, L=150$ Byres, $S_{A}=10 \mathrm{~ms}, S_{B}=15 \mathrm{~ms}$

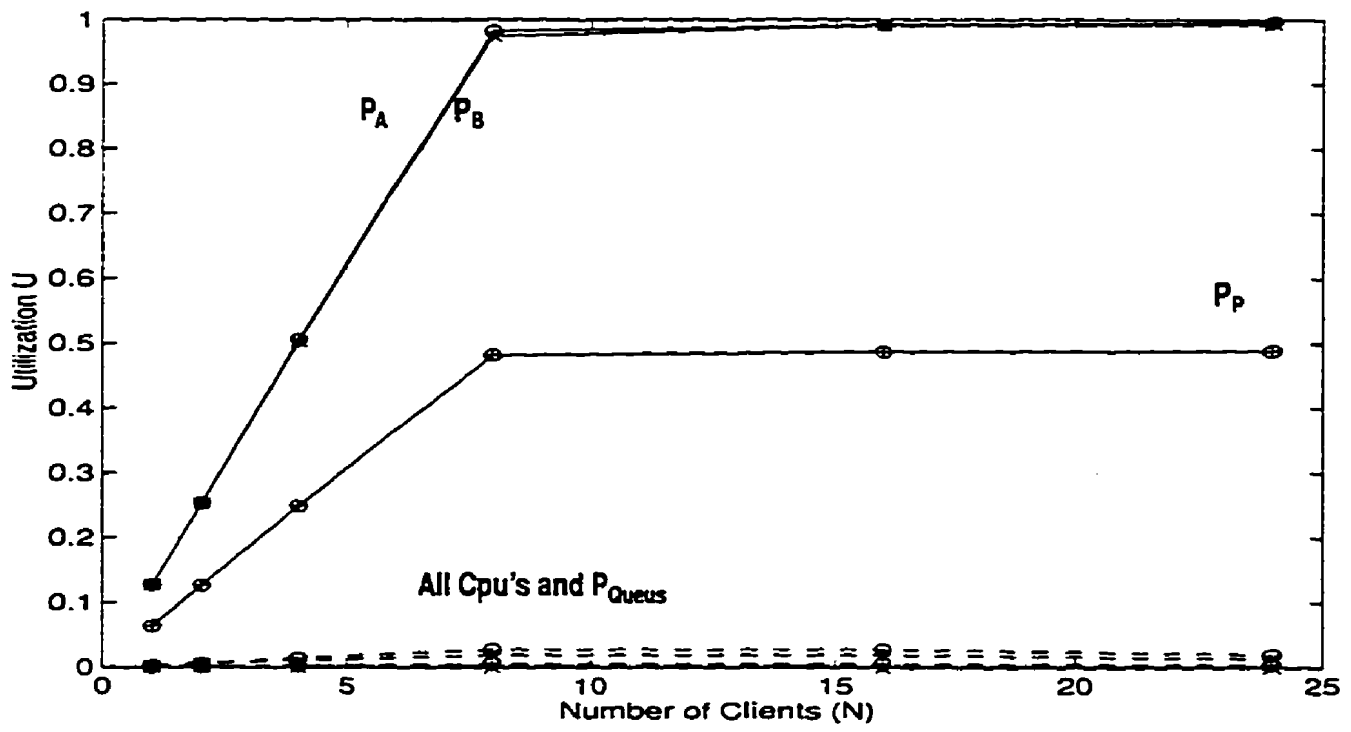

Figure C-29: Cpu \&Process Utilizations of the $P-O R B$ with $D=1000 \mathrm{~ms}$, and 8 Cloning degrees.

$P_{A}=$ Process Util. of Server $A, C P U_{A}=C p u$ Util of Server $A, P_{P}=$ Process Util. of p-agent. PQueures $=$ Process Util. of dispatcher, Degree of Cloning $=8, D=1000 \mathrm{~ms}, L=150$ Bytes, $\mathrm{S}_{\mathrm{A}}=10 \mathrm{~ms}, \mathrm{~S}_{\mathrm{B}}=15 \mathrm{~ms}$ 
Appendix D: Supplementary Results for the A-ORB Set of

\author{
Experiments
}




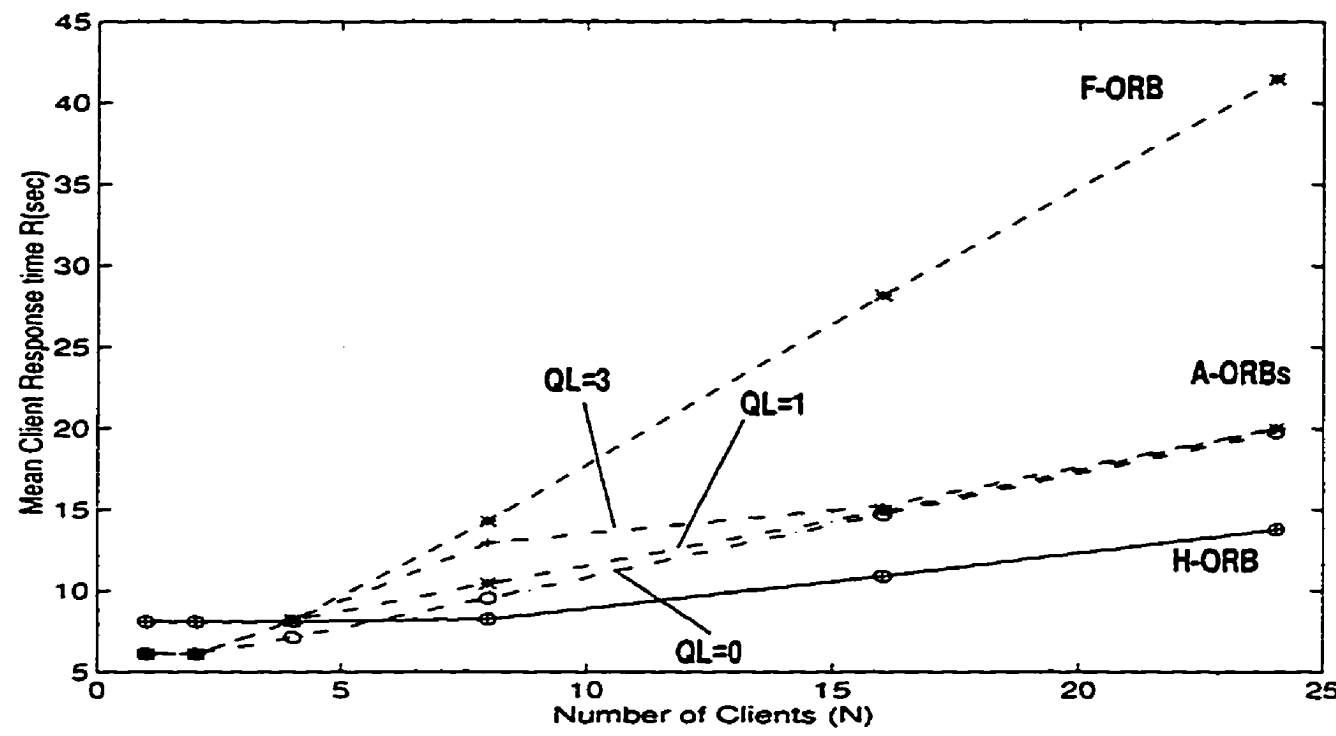

Figure D-1: The Response Time of the H-ORB, the F-ORB and the A$O R B$ with Equidistant Nodes, and $D=1000 \mathrm{~ms}$.

Degree of Cloning $=1$, Del=1000 ms, $L=150$ Bytes, Server $A=10 \mathrm{~ms}$, Server $B=15 \mathrm{~ms}$. Queue Length $=0,1,3$.

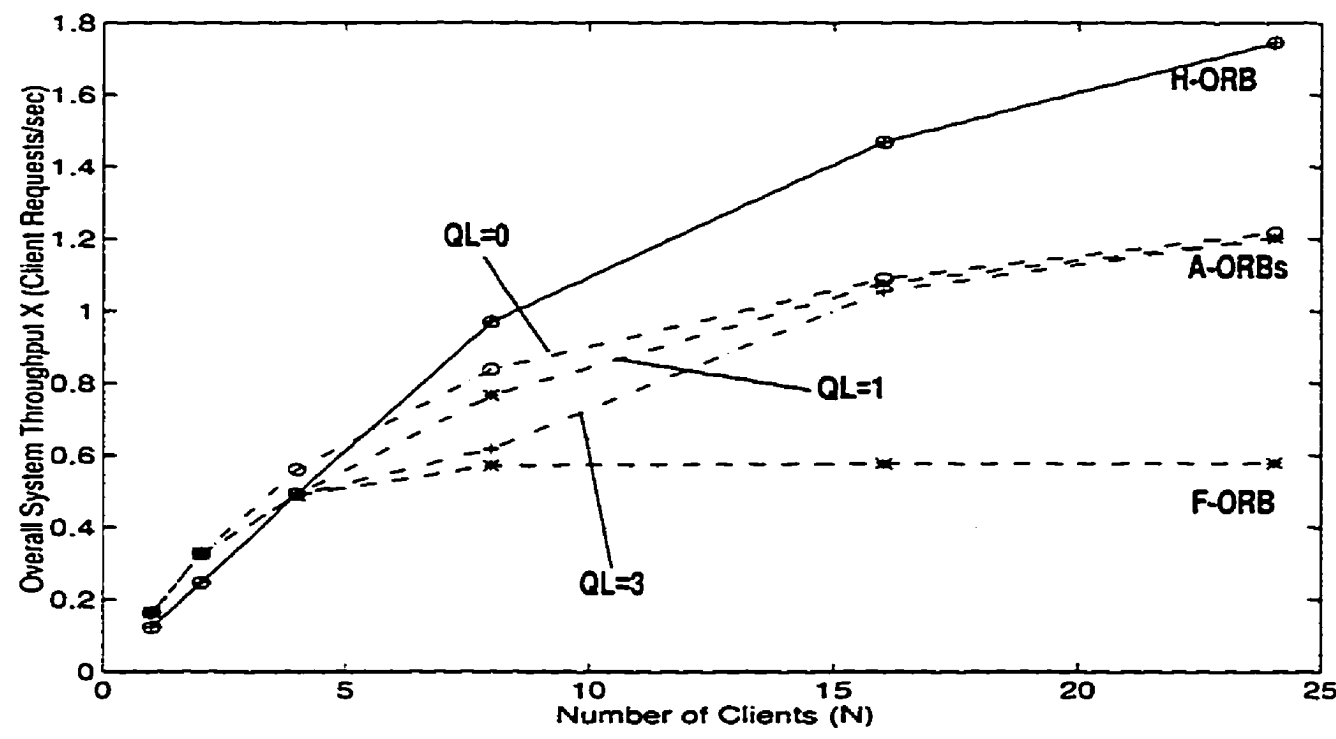

Figure D-2: The Throughput of the H-ORB, the F-ORB and the A-ORB with Equidistant Nodes, and $D=1000 \mathrm{~ms}$.

Degree of Cloning $=1$, Del $=1000 \mathrm{~ms}, \mathrm{~L}=150$ Byres, Server $A=10 \mathrm{~ms}$, Server $B=15 \mathrm{~ms}$. Queue Length $=0,1,3$. 


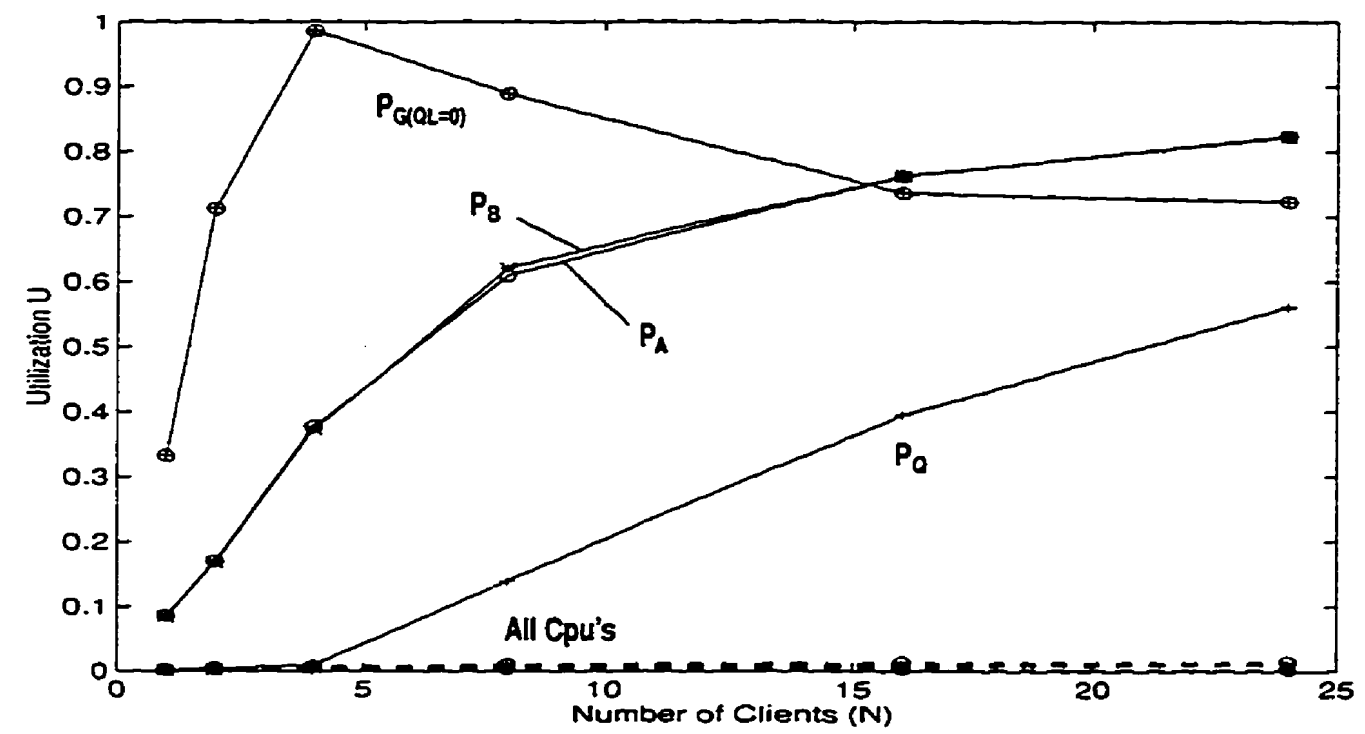

Figure D-3: The Process and Cpu Utilizations of the A-ORB with Equidistant Nodes, and $D=1000 \mathrm{~ms}$.

$P_{A}=$ Process Util of Server $A, C P U_{A}=C p u$ Util of server $A, P_{G}=$ Process Util of a-agent. $P_{Q}=$ Process Util of Queue, Degree of Cloning $=1$, Del $=1000 \mathrm{~ms}, L=150$ Bytes, $S_{A}=10 \mathrm{~ms}$, $S_{B}=15 \mathrm{~ms}$, Queue Length $=0$.

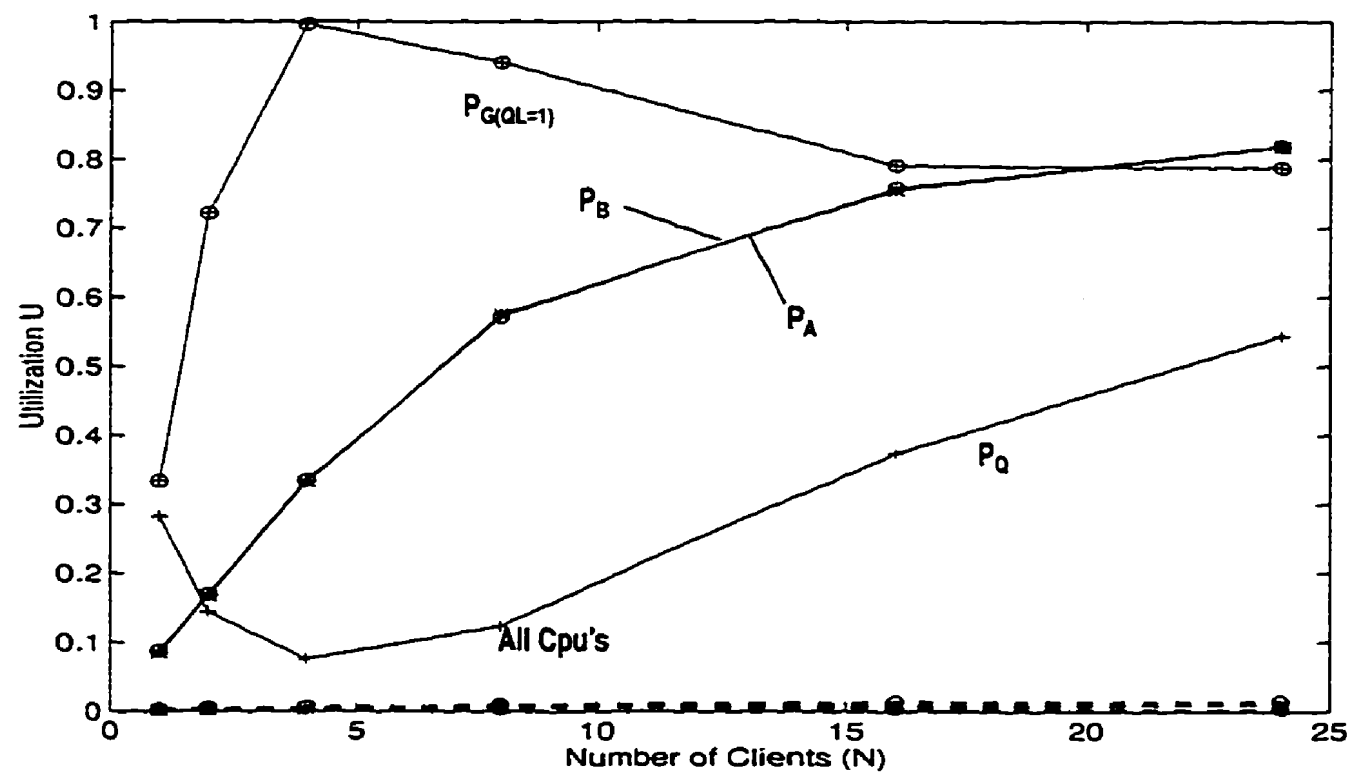

Figure D-4: The Process and Cpu Utilizations of the A-ORB with Equidistant Nodes, and $D=1000$ ms.

$P_{A}=$ Process Util of Server $A, C P U_{A}=$ Cpu Util of server $A, P_{G}=$ Process Util of a-agent, $P_{Q}=$ Process Util of Queue, Degree of Cloning $=1$, Del $=1000 \mathrm{~ms}, L=150$ Bytes, $S_{A}=10 \mathrm{~ms}$, $\mathrm{S}_{\mathrm{B}}=15 \mathrm{~ms}$, Queue Length $=1$. 


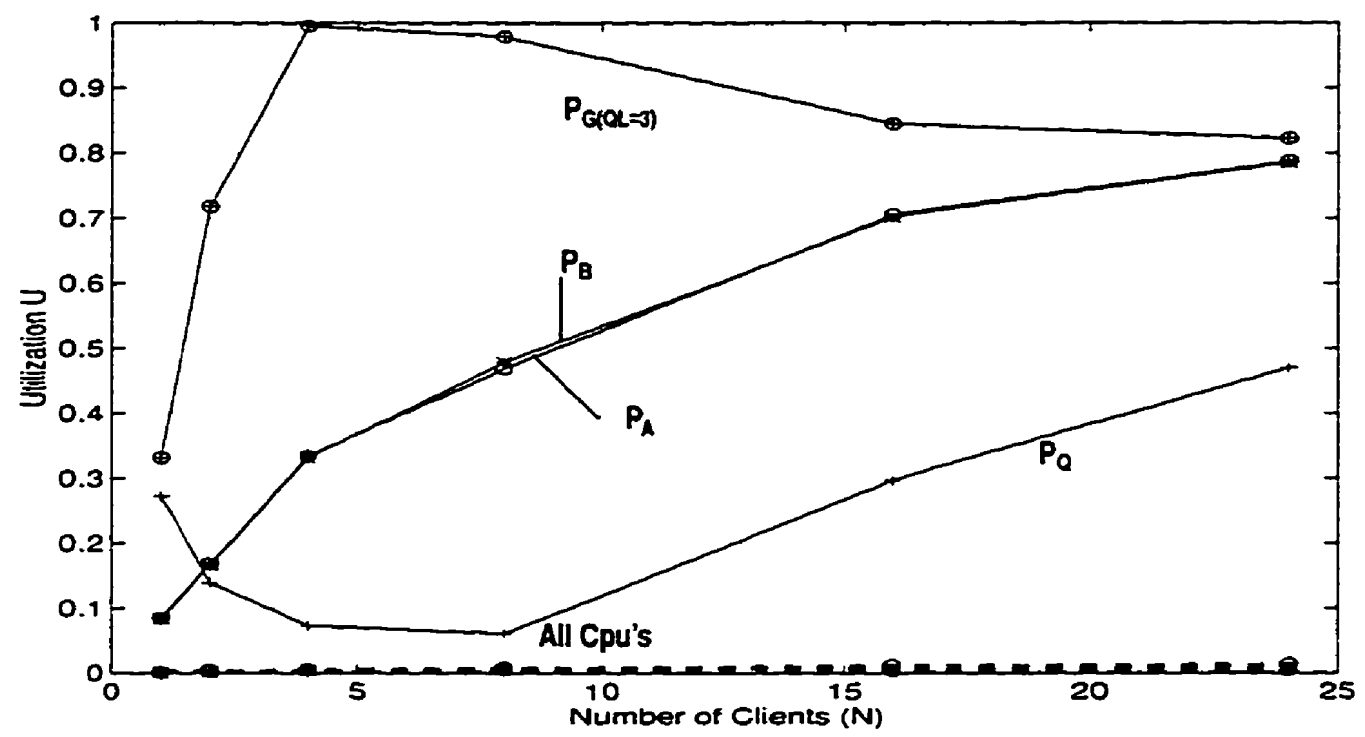

Figure D-5: The Process Utilization of the A-ORB with Equidistant Nodes, $D=1000$ ms.

$P_{A}=$ Process Util of Server $A, C P U_{A}=C p u$ Util of server $A, P_{G}=$ Process Util of a-agent, $P_{Q}=$ Process Util of Queue, Degree of Cloning $=1$, Del $=1000 \mathrm{~ms}, L=150$ Byres, $S_{A}=10 \mathrm{~ms}$, $\mathrm{S}_{\mathrm{B}}=15 \mathrm{~ms}$, Queue Length $=3$. 